\title{
Spatial Cue-Priming: Effects of Masked Cue Stimuli on \\ Endogenous Visual Spatial Attention
}

\author{
Dissertation \\ zur Erlangung des mathematisch-naturwissenschaftlichen Doktorgrades \\ "Doctor rerum naturalium" \\ der Georg-August-Universität Göttingen \\ im Promotionsprogramm Biologie \\ der Georg-August University School of Science (GAUSS)
}

vorgelegt von

Simon Palmer

aus Braunschweig

Göttingen, 2013 


\section{$\underline{\text { Betreuungsausschuss }}$}

Prof. Dr. Uwe Mattler (Abt. Experimentelle Psychologie, GEMI für Psychologie)

Prof. Dr. Uta Lass (Abt. Experimentelle Psychologie, GEMI für Psychologie)

Mitglieder der Prüfungskommission:

Referent: Prof. Dr. Uwe Mattler (Abt. Experimentelle Psychologie, GEMI für Psychologie)

Koreferentin: Prof. Dr. Uta Lass (Abt. Experimentelle Psychologie, GEMI für Psychologie)

Weitere Mitglieder der Prüfungskommission:

Prof. Dr. Roland Grabner (Abt. Pädagogische Psychologie, GEMI für Psychologie)

Prof. Dr. Nivedita Mani (Free Floater Nachwuchsgruppe - Spracherwerb)

Prof. Dr. Hannes Rakoczy (Abt. Biologische Entwicklungspsychologie, GEMI für Psychologie)

Prof. Dr. Andreas Glöckner (Abt. Psychologische Diagnostik, Urteilen und Entscheiden, GEMI für Psychologie)

Tag der mündlichen Prüfung: 01.03.2013 


\section{Acknowledgements}

This work profited from support of the following people. I am very grateful for their help.

Uwe Mattler provided ideas, knowledge, supervision, and perspective. Without his support, this work could not have been done.

Uta Lass took in on her to review the present thesis and provided an unwavering sense of optimism.

Susan Klapötke, Martina Wernicke, Thorsten Albrecht, and Daniel Krüger provided professional input, emotional support and a pleasant atmosphere to work in.

Nadja Arnold, Franziska Liedtke, Nils Müller, and especially Tobias Talanow provided invaluable and reliable help in collecting the data.

Göttingen, March 2013 
This work contains three manuscripts that were submitted for publication in peer reviewed journals. These manuscripts were slightly modified to fit the structure of the present document but are otherwise complete, and are included as Sections 2.1, 3.1, and 4.1. This necessarily leads to some redundancy in the sections on theory and the discussions. Whenever there were references in one of the manuscripts to one of the other manuscripts included in this work, they were replaced with a reference to the corresponding project. References have been combined to one complete list of references at the end of this work. 


\section{Summary}

Recent research in cognitive neuroscience has examined the relationship of consciousness and attention. On the one hand, it remains a debated topic whether attention is a necessary precondition for consciousness; on the other hand other researchers have focused on the possibility and mechanisms of attention without consciousness. Effects of unconscious stimuli on spatial attention were found in several studies but were so far restricted to peripheral cues or specific central cues which may invoke exogenous attention. Given that recent evidence suggests that unconscious stimuli have access to executive control processes, it seemed reasonable to assume that masked stimuli can affect endogenous orienting of attention as well. In the present work, masked primes were presented before visible endogenous cues in different spatial cueing tasks. These masked primes modulated speed and accuracy of responses to laterally presented visual target stimuli. This suggests that they affected endogenous orienting of attention. However, the effects of these masked primes are modulated by several factors such as perceptual similarity between prime and cue stimuli, spatial compatibility between primes and target location, validity of the visible cue stimulus, and whether the task and target parameters favor attentional selection at early or later stages of processing.

Overall, results suggest different underlying mechanisms in spatial cue-priming. Large and reliable spatial cue-priming effects were found in letter discrimination tasks in which visible cues specified the target letter. In this task, priming effects on behavior seem to result in part from perceptual priming of cues and in part on later decisional processes. Direct effects of primes on attention were found in free choice task but were restricted to spatially compatible stimuli. Cue-priming effects on attention at early levels of processing seem to be mainly based on perceptual priming of cue processing. In EEG-experiments, a modulation of 
the visual N1 component provided further evidence for cue-priming effects on early levels of processing. In sum, results show that endogenous spatial attention can be affected by masked stimuli at different stages of target processing. However, further research is needed to clarify the mechanisms and limits of these spatial cue-priming effects. 


\section{Contents}

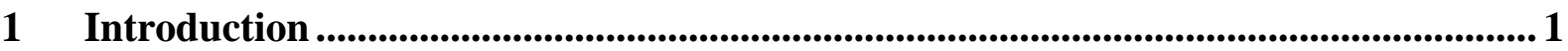

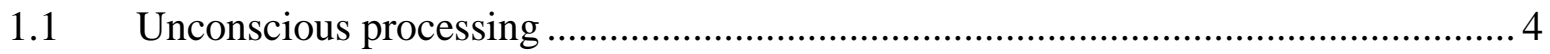

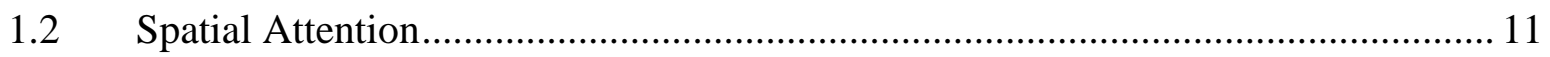

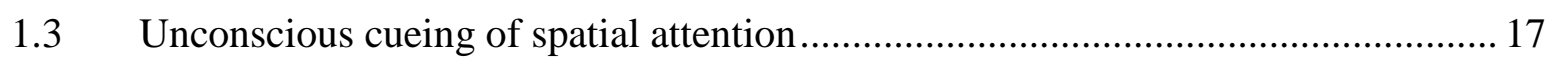

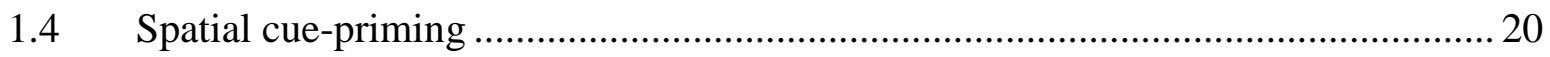

2 Project 1 - Spatial cue-priming of letter discrimination .......................................... 22

2.1 Masked stimuli modulate endogenous shifts of spatial attention............................ 22

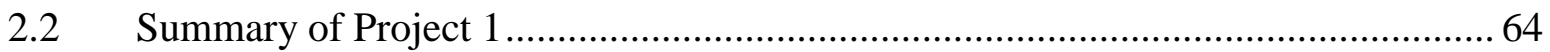

3 Project 2 - Cue-priming effects on accuracy in signal detection tasks .................... 67

3.1 Experiments 4 and 5 - On the source and scope of priming effects of masked stimuli on endogenous shifts of spatial attention'

3.2 Experiments 6 and 7 - Preconditions for spatial cue-priming in signal detection tasks 105

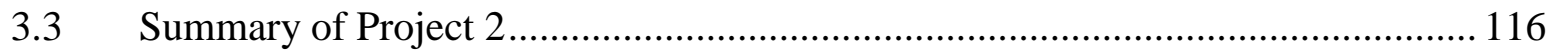

4 Project 3 - Spatial cue-priming effects on physiological measures of target

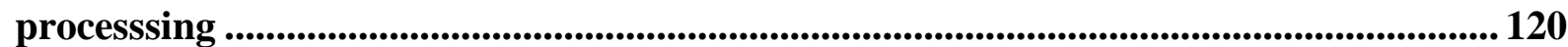

4.1 Experiment 8 - Masked Stimuli Modulate Endogenous Spatial Attention at Early Levels of Target Processing ........................................................................................ 120

4.2 Experiment 9 - Priming effects on stimuli presented at non-cued locations .......... 144

4.3 Experiment $10-$ The role of cue-target SOA and distractor presence................... 160

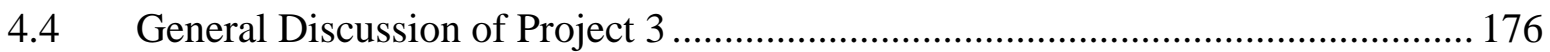

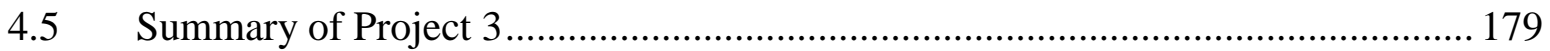

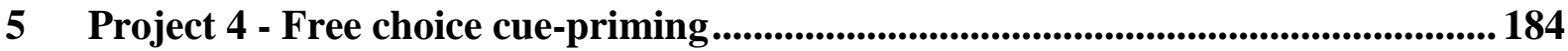

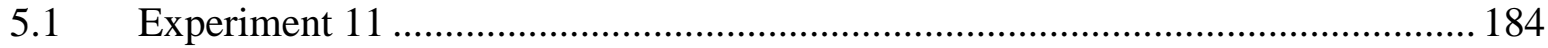

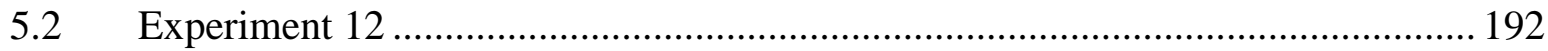

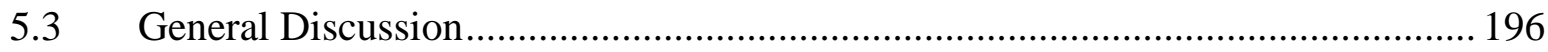

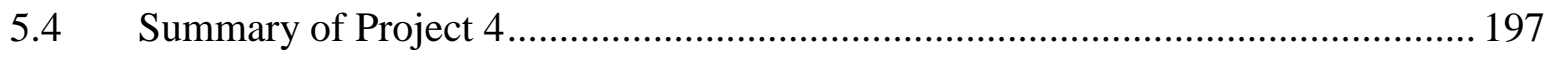


6 Project 5 - The role of cue validity in spatial cue-priming ......................................... 199

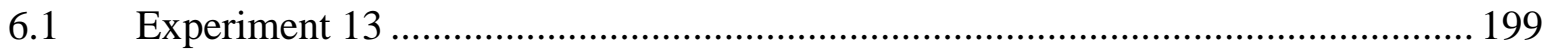

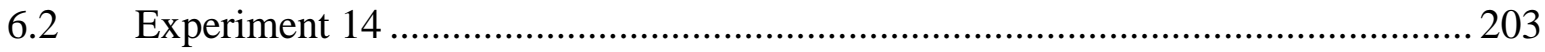

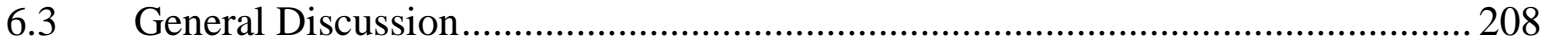

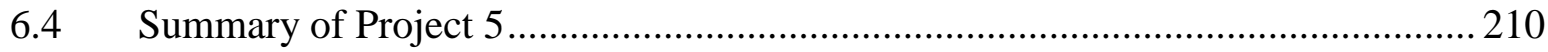

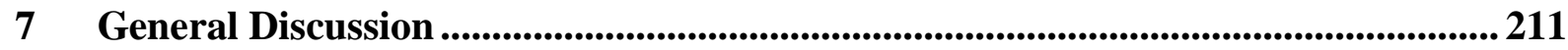

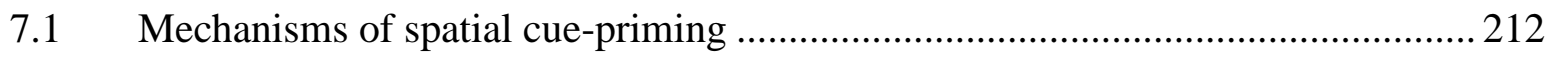

7.2 Alternative accounts for cueing effects in the present experiments ...................... 218

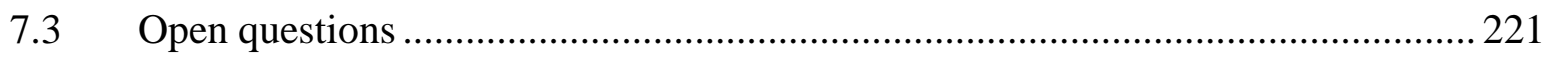

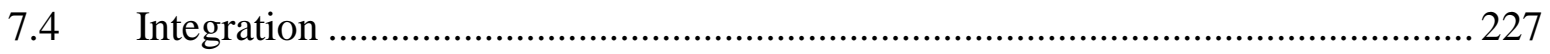

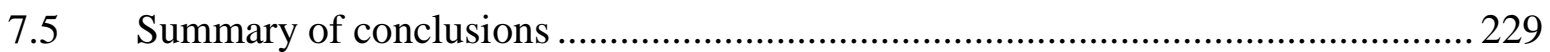

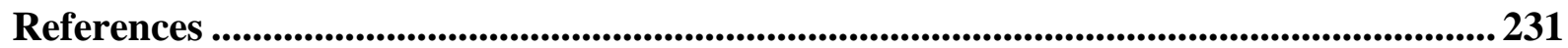

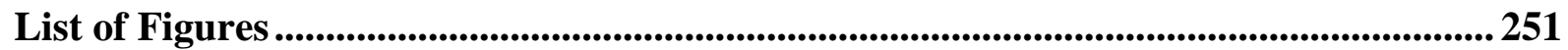

List of Tables.............................................................................................................................. 253

Curriculum Vitae .................................................................................................................................. 254 


\section{Introduction}

Consciousness has become a widely discussed topic in cognitive science. One aspect of this discussion is the question about the function of consciousness in processing of information. Related to this, is the question to what extent human behavior can be affected by unconscious or subliminal stimuli. This issue was made popular by fraudulent claims of effective subliminal advertising (Pratkanis, 1992). James Vicary asserted in 1958 that he was able to boost sales of popcorn and soft drinks by presenting very short messages of which the audience was unaware during movies. His method did not receive scientific substantiation initially, but related results from more credible sources (Bermeitinger, Goelz, Johr, Neumann, Ecker \& Doerr, 2009; Karremans, Stroebe, \& Claus, 2006) show that this issue has not settled and the issue of subliminal advertising is still present in public opinion.

The question whether unconscious or subliminal stimuli can affect human behavior has been asked in psychological research for a long time. The notion was often met with skepticism, as it conflicts with human's subjective experience that we control our behavior and have access to all relevant sensory information. However, growing evidence collected over the last decades suggests that unconscious influences on behavior do exist. Research goals have shifted from trying to provide evidence for unconscious perception to trying to determine the limits of unconscious information processing. Early evidence for unconscious perception suggested that subliminal stimuli can activate relatively simple processes like feedforward activation of motor responses in specific tasks (Neumann \& Klotz, 1994). Over time, claims of unconscious influences have extended to more complex processes, like activation of semantic networks (Dehaene et al., 1998), influencing executive control processes such as task switching (Lau \& Passingham, 2007; Mattler, 2003b; Reuss, Kiesel, Kunde \& Hommel, 2011) and response inhibition (van Gaal, Ridderinkhof, Fahrenfort, Scholte \& Lamme, 2008; 
van Gaal, Ridderinkhof, Wildenberg \& Lamme, 2009), directly activating social behavior (Bargh, Chen \& Burrows, 1996) or goals (Custers \& Aarts, 2010). One area of research that has links to the study of consciousness and unconscious processing is the study of attention. Classically, attention has been associated with consciousness in several ways. On the one hand, attention has been hypothesized to be necessary for conscious perception; on the other hand, the allocation of attention to specific stimuli can be under conscious control.

"Everyone knows what attention is. It is the taking possession by the mind, in clear and vivid form, of one out of what seem several simultaneously possible objects or trains of thought. Focalization, concentration, of consciousness are of its essence. It implies withdrawal from some things in order to deal effectively with others...” (James, 1950, p. 381). The above quote hints at a strong connection between consciousness and attention. Everyone knows what attention is because subjective experience suggests that we can control this filtering of information to some degree in order to select relevant components. Thus, attention seems to be under voluntary or conscious control. Classically, consciousness and attention have been conceived to be associated (e.g., Posner, 1994). Recently, however, it has been suggested that attention and consciousness can be better conceptualized as independent processes (Koch \& Tsuchiya, 2007) and there is an ongoing debate about this topic (Cohen, Cavanagh, Chun \& Nakayama, 2012; Tsuchiya, Block \& Koch, 2012). Presently, this debate focuses more on the question whether consciousness is possible without (top-down-) attention. The complementary question whether consciousness plays a role in the direction of top-down attention remains largely unexplored.

Possibly, the most studied area in the field of attention is the direction of visual attention in space. Here, two different ways of directing attention have been identified. One endogenous orienting being associated with voluntary, top-down controlled processing; the 
other - exogenous orienting - being bottom-up driven by external stimulation and rather automatic (Jonides, 1981).

Several studies have reported effects of subliminal stimuli on spatial attention. This has been found for subliminal peripheral sudden onset stimuli (McCormick, 1997; Scharlau \& Ansorge, 2003) as well as symbolic stimuli that are known to direct attention involuntarily, such as arrows (Cole \& Kuhn, 2010) and gaze direction (Sato, Okada \& Toichi, 2007). In contrast, no effects of subliminal stimuli on voluntary direction of attention have been reported and it has been suggested that directing attention according to symbolic stimuli requires conscious control: “(...) voluntary attention is conscious control of access to consciousness" (Baars, 1993, p. 305).

Combining the research on cueing of spatial attention with methods from research on effects of subliminal stimuli, we try to shed new light on the role of consciousness in spatial cueing with symbolic stimuli, which involves endogenous directing of attention. To anticipate results, masked primes can affect spatial attention. Based on this finding, a large part of the experiments focuses on clarifying the cognitive mechanisms causing this effect. Here, two important questions can be distinguished: (1) by which mechanisms do primes affect spatial attention? (2) are there differences between cue-priming at different levels of target processing or can all levels of attentional selection be affected by masked primes? These questions cannot be finally answered based on the present results, but progress towards potential solutions was made.

Two lines of research are relevant for the present study. On the one hand, the assumed limits of unconscious processing have been increased by recent evidence. On the other hand, a rich tradition of research on visual spatial attention has provided insight into the mechanisms by which spatial attention can be directed as well as the mechanisms by which attention 
selects relevant information. The following introduction focuses first on evidence for unconscious processing then provides an overview of relevant issues in spatial attention focusing in particular on the direction of attention according to unconscious cues. These two lines of research provide the basis for the present experiments and for the spatial cue-priming method which was used in all experiments and is introduced in the final section of the introduction.

\subsection{Unconscious processing}

Unconscious stimuli have been found to affect several cognitive processes such as motor processing (Klotz \& Neumann, 1999; Vorberg, Mattler, Heinecke, Schmidt \& Schwarzbach, 2003), semantic processing (Dehaene et al. 1998), response inhibition (Van Gaal, Ridderinkhoff, Scholte \& Lamme, 2008), and task preparation (Mattler, 2003; Lau \& Passingham, 2007). Of particular relevance to the present work are effects on spatial attention and executive control processes. In addition, the cue-priming paradigm employed in the present experiments was derived from response priming. Thus, cue-priming and response priming may share several characteristics and theoretical aspects. This warrants a short discussion of findings on the priming of motor responses by masked stimuli.

\subsubsection{Response Priming}

Among the first studies to convincingly demonstrate unconscious perception were studies that found effects of masked stimuli on motor responses (Vorberg, et al., 2003; Neumann \& Klotz, 1994; Klotz \& Neumann, 1999; Klotz \& Wolff, 1995; Schmidt, 2002). In these experiments participants usually perform simple speeded discrimination tasks between two possible target stimuli. Replicas of these target stimuli are presented before the targets and, albeit being masked, can activate the corresponding response. In the experiments of 
Vorberg et al. (2003), for instance, subjects had to indicate the direction of a target arrow by pressing corresponding buttons as fast and as accurately as possible. Unbeknownst to them, each target was preceded by a prime stimulus. These primes were smaller left or right pointing arrows that were metacontrast masked by the targets, as they fit exactly in the cutouts in the center of the targets. On half of the trials, prime and target were pointing in the same direction (congruent); in the other half they were pointing in different directions (incongruent). The pattern of results obtained in the experiments was that subjects' responses were faster and more accurate on congruent trials than on incongruent trials. The difference between these two types of trials results from effects of the primes. Clearly, primes are effective because they have critical features that define target stimuli.

Early explanations featured a theory of direct parameter specification, which assumed that one parameter of a response can be specified directly by sensory evidence if all other parameters have been set in advance (Neumann \& Klotz, 1994), without having to be subject to higher potentially conscious processing. A related account was proposed by Kunde, Kiesel and Hoffman (2003). These authors proposed that participants pre-specify action triggers in a given task and stimuli that sufficiently match the conditions for one of these will immediately activate the corresponding action even in the absence of awareness. Motor activation induced by subliminal primes was indeed found in lateralized response potentials (Leuthold \& Kopp, 1998) as well as in overt behavior (Schmidt, 2002; Schmidt, Niehaus \& Nagel, 2006). Vorberg et al. (2003) explained response priming with an accumulator model that assumes that primes are processed in the same way as targets. In their model two neuronal accumulators accumulate evidence for either a left or a right response. Responses are initiated when the difference in activation between both accumulators reaches a threshold. As primes and targets share defining features they are equally effective in providing evidence. Primes 
reside in a sensory buffer and provide evidence for the associated response until the target is presented. This introduces a bias in the accumulators for the primed response. Thus, on incongruent trials primes drive the accumulator difference away from the threshold for the correct response, whereas they drive it towards the threshold on congruent trials. Then, when evidence provided by the target drives the accumulators the threshold is reached faster on congruent than on incongruent trials. On some trials, prime induced accumulator activation suffices to reach the threshold which explains frequent response errors on incongruent trials. One finding that this model accounts for very well is that priming effects increase linearly with the time that passes between presentation of prime and target (stimulus onset asynchrony - (SOA). The response priming paradigm has also been used to show that masked primes have access to motor processing (Leuthold \& Kopp, 1998; Schmidt, 2002) and can bias free choices between two response alternatives (Schlaghecken \& Eimer, 2004; Mattler \& Palmer, 2012; Kiesel et al., 2006). However, evidence for such direct effects of primes on behavior comes primarily from studies using spatially compatible stimuli, and recent evidence suggests that spatial correspondence between stimuli and responses might play an important role for prime induced motor activation (Mattler \& Palmer, 2012).

\subsubsection{Unconscious effects on executive control processes}

Recently, unconscious processing has been found to affect more complex processes and the notion that executive control processes require awareness has been called into question, both theoretically (Hommel, 2007) and empirically in studies that showed effects of subliminal stimuli on task preparation and directing of attention (Lau \& Passingham, 2007; Mattler, 2003, 2007) and response inhibition (van Gaal et al., 2009, 2008). Van Gaal and Lamme (2012) assumed that unconscious processing is indicative of a feed-forward sweep 
and unconscious effects on executive control can be taken as evidence that feed-forward processing can reach prefrontal areas.

One method that has been used to study effects of subliminal stimuli on processes more complex than the selection of motor responses is the cue-priming paradigm first used by Mattler (2003). The basic idea is similar to response priming in that a prime is presented before a critical stimulus. Just like in response priming, the prime can be either congruent or incongruent. The difference is that in cue-priming the critical stimulus - the cue - does not specify a motor response but a mental operation to be executed on a third stimulus - the target. For instance, Mattler (2003) showed priming effects of metacontrast-masked stimuli in several cueing tasks thought to involve executive control processes. In Experiment 3 of his study, subjects were instructed to shift their attention to either the auditory or visual modality according to a symbolic cue stimulus (a square or a diamond). Unbeknownst to them, this cue stimulus was always preceded by a masked prime, which was also either a square or a diamond. When the cue and the prime had the same shape, subjects were faster to identify a target stimulus in the cued modality than when they were of different shape. This was interpreted as evidence that subliminal primes can affect shifts of attention between modalities. Similar effects were found when cues indicated which hand to respond with or whether to discriminate pitch or timbre of a target sound. Essentially, these cue-priming experiments are very similar to response priming experiments with the difference that primes do not signal which response to execute, but instead to prepare for a specific cognitive process or mental operation. Mattler (2005) later showed that these effects are rather short lived and decay with increasing cue-target SOA. Lau und Passingham (2007) adopted Mattler's cuepriming task in an fMRI setting. They used a different task in which cues indicated whether subjects were to indicate whether a target word had one or more syllables (phonological task) 
or was concrete or abstract (semantic tasks). Both tasks were associated with neural activity in specific brain regions which allowed them to find that primes modulate neural activity in brain areas associated with the respective task. Most importantly, they found that neural activity in areas associated to the primed task is induced even when it is task irrelevant, i.e. not cued by the visible cue stimulus. Moreover, a region in left dorsolateral prefrontal cortex was more active in incongruent than in congruent trials. This was interpreted as evidence that primes can activate cognitive control processes. Similar results were obtained in another study that combined cue-priming with fMRI (Klapötke, 2011). Here, cues indicated the relevant aspect of a complex picture stimulus consisting of a scene and a face. Subjects should either indicate whether the face was male or female or whether the scene was an interior or exterior scene. A third fMRI study of cue-priming with yet another task was recently conducted by De Pisapia, Turatto, Lin, Jovicich und Caramazza (2011). They used lateral visual stimuli as targets and cues indicated whether the correct response was ipsilateral or contralateral to the target. On incongruent trials, they found increased activity in executive networks accompanied by a decrease in activity in default-mode networks. This was interpreted as being due to increased need for conflict resolution and thus evidence that subliminal stimuli can affect executive control processes. Additionally, their experiment featured a condition with neutral primes. Compared to this baseline condition primes seemed to induce costs rather than benefits, as there was no difference in RT between congruent and neutral trials but responses were slower on incongruent trials.

A possible reason why endogenous shifts of attention might require visible cue stimuli is the notion that they require executive control and an intentional shift away from the cue. Given that these studies all used central symbolic cues and the fact that there is evidence suggesting that spatial attention shifting shares mechanisms with other kinds of attentional 
preparation similar to those examined in cue-priming studies (Chiu \& Yantis, 2009; Slagter, Kok, Mol \& Kenemans, 2005), it seems interesting to transfer Mattler's cue-priming paradigm to a task involving spatial attention in order to clarify whether subliminal symbolic cues can affect orienting of spatial attention in a similar manner, even when they are presented below an objective threshold of awareness. If masked primes can indeed affect endogenous shifts of attention a possible mechanism might be that they trigger the same processes as visible cues. This would call into question the notion that spatial cueing with central symbolic cues always requires awareness of cueing stimuli. Furthermore, if performance in a spatial attention task is affected by subliminal stimuli in the same way as in other tasks, priming effects should exhibit similar time courses when SOAs between the critical stimuli are varied. This would increase the amount of evidence that cue-priming is domain independent.

Similar cue-priming effects have been found for different types of tasks: cueing part of a motor response, cueing the modality of a target stimulus (auditory or visual), cueing task relevant attributes of multidimensional target stimuli (pitch vs. instrument (Mattler, 2003, 2005), scene vs. face (Klapötke, 2011), number of syllables vs. semantic category (Lau \& Passingham, 2007) and the mapping of stimuli to motor responses (De Pisapia et al., 2011). Cue-priming effects were similar in all tasks in that they increased with prime-cue SOA, decreased with cue-target-SOA (only varied by Mattler, 2005) and were independent of prime visibility. Cue-priming tasks are similar to motor priming tasks in that masked primes share attributes with another task relevant stimulus (target in motor priming, cue in cue-priming). In addition, both priming effects seem to increase with SOA between prime and the relevant stimulus and are independent of prime visibility. This could be taken as evidence that both effects are based on similar mechanisms. The accumulator model of motor priming (Vorberg 
et al., 2003) could explain cue-priming effects if, instead of evidence for motor responses, evidence for tasks or modes of attention is accumulated (Klapötke, 2011). In cue-priming, similar processes as in response priming, e.g., direct parameter specification might occur at a higher level.

Extending the cue-priming paradigm to a spatial cueing task potentially allows conclusions about the underlying mechanisms of cue-priming. Mattler (2003; see also Klapötke, 2011) proposed three possible loci for cue-priming effects: a perceptual locus, a central locus or a post-central locus. As described in Section 2.1, a comparison of spatial and non-spatial cue-priming might yield insights in the generalizability of the effect. Furthermore, the spatial task allows control and variation of several target parameters like position, contrast, distractors and number. Investigating effects of these parameters in cue-priming bears the potential to discern potential loci and mechanisms.

\subsubsection{Prime visibility}

Historically, in research of unconscious perception, the burden of proof has been on proponents of such unconscious effects to show that their effective stimuli were indeed subliminal. This raises the important question when a stimulus can be considered subliminal. Early studies distinguished a subjective threshold at which participants report not to be able to discriminate stimuli from an objective threshold at which they actually cannot discriminate stimuli when forced to guess (Cheesman \& Merikle, 1984). Usually, in priming studies, prime visibility is measured objectively in a separate task. Subjects are informed about primes and are asked to discriminate them, usually without timing restrictions. This method provides an objective measure of participants' ability to discriminate the primes which is then interpreted as a conservative measurement of their awareness of the primes: if subjects cannot perform better than chance at this forced choice discrimination task it is unlikely that they have any 
conscious perception of the primes. Thus it can be concluded that primes are too weak to reach the objective threshold of visibility. However, proof for the absolute absence of prime visibility is difficult to obtain as it requires acceptance of a null hypothesis.

Other studies have used a different method to show independence of priming effects of prime visibility by showing double dissociation of the two measures (Vorberg et al., 2003; Mattler, 2003; Lau \& Passingham, 2007; Klapötke, 2011; Albrecht, Klapötke \& Mattler, 2010). Under certain circumstances, metacontrast masking is more effective at longer than at shorter SOAs whereas response priming and cue-priming effects increase with prime-cue SOA. Thus, increasing prime-cue SOA leads to larger priming effects and decreased prime visibility at the same time. This has been taken as evidence that both measures cannot be based on the same underlying process (Schmidt et al., 2006). This double dissociation of priming effects and prime visibility has also been found in cue-priming tasks (Mattler, 2003; Klapötke, 2011; Lau \& Passingham, 2007). With more and more proof for effects of unconscious stimuli, requirements to prove subliminality seem to have lowered somewhat. Extraordinary claims require extraordinary evidence, but claims of unconscious perception are hardly extraordinary any more.

\subsection{Spatial Attention}

The term attention describes mechanisms that allow us to select relevant information from the vast amount of sensory input we receive. Information can be selected according to multiple attributes and probably the most studied mode is the selection of visual input by where it occurs in space. 


\subsubsection{Mechanisms of spatial attention}

The purpose of attention is to select behaviorally relevant stimuli from an abundance of incoming information. Selection is possible at several stages of stimulus processing. Classic theories have located selection at different stages of processing. Early selection theories assume that attention operates at early perceptual levels whereas late selection theories locate attentional effects at post perceptual levels. According to the early selection account, unattended stimuli are not fully perceived whereas late selection accounts assume parallel processing of all stimuli with attention acting later to select the correct response. Considerable evidence for both early and late selection has been collected, indicating that neither account explains attentional selection entirely and that more complex models are required.

An old controversy in research on attention has focused on the question at which stage of processing attention operates. A distinction can be made between early selection accounts (Broadbent, 1958; Kahneman \& Treisman, 1984; Laberge \& Brown, 1989) and late selection accounts (Deutsch \& Deutsch, 1963; Duncan, 1980; Shaw, 1984). Both accounts assume that attention is needed to select information because of capacity limitations but differ in their assumptions at which stage of stimulus processing these limitations play a role. Whereas proponents of early selection assume that filtering occurs at early sensory stages, i.e. before stimuli are identified as objects, proponents of late selection assume that early perceptual information is processed in parallel at early stages but there are capacity limits when stimuli are operated on, for instance when a response, semantic categorization, or storage in memory is required.

The perceptual load theory of attention (Lavie \& Tsal, 1994) assumes that the locus of selection depends on the amount of perceptual load. Only under conditions of high perceptual load will selection operate at early stages. In a similar vein, Luck \& Hillyard (2000) proposed 
that attentional selection occurs at those processing stages at which interference is induced by task demands. Perceptual interference, favoring early selection, occurs when stimuli are presented with such low intensity that their processing is significantly affected by neural noise. However, under different conditions attention can select for entrance into working memory, e.g., the attentional blink paradigm or response execution, e.g., PRP-Paradigm. In summary, spatial attention can affect processing of target stimuli at multiple stages and a strict separation of early and late selection is probably not useful. Nonetheless, it might be that access to certain levels of processing is restricted and masked stimuli might only be able to affect a subset of attentional processes. Thus, it seems warranted to study the effects of masked stimuli on spatial attention in several tasks, varying attentional demands. The spatial cueing paradigm offers the opportunity to do that, because task difficulty can easily be varied by varying target and distractor stimuli.

\subsubsection{Spatial Cueing}

In spatial cueing (Posner, 1980; Posner, Cohen \& Rafal, 1982; Jonides, 1981) subjects shift their attention according to a given cue stimulus. This cue stimulus is usually informative about the location of a target stimulus, meaning that the target stimulus is more likely to appear at the cued location than at a different location. However, in most studies cues are not always valid and finding differences in processing between validly cued targets and invalidly cued stimuli is often the rationale of these studies. In contrast to earlier studies on sustained attention, in which subjects were asked before each block of an experiment to attend to a certain location, this method allows to examine effects of the shifting of attention. Spatial cueing was used to investigate the time course of attention shifting (e.g., Müller \& Rabbitt, 1989) and to differentiate between costs and benefits of attending to a certain location by comparing valid and invalid cues to neutral cues (e.g., Posner, 1980). Cue stimuli can either 
be presented centrally, affecting attention because subjects interpret them as standing for a specific location or peripherally, drawing attention to their location. Especially with central cues, cueing effects seem to depend on perceptual attributes of the cue stimuli. For instance, arrow and eye gaze stimuli might be especially potent in directing attention because they are associated with directing attention from everyday learning (Guzzon, Brignani, Miniussi \& Marzi, 2010). It might also be that covariation of a specific feature within the stimulus and target location greatly enhances cueing effects by a process called spatial correspondence learning (Lambert \& Duddy, 2002).

\subsubsection{Endogenous vs. exogenous orienting of attention}

Two mechanisms that can direct spatial attention have been distinguished both functionally (Jonides, 1981) and anatomically (Corbetta \& Shulman, 2002), one has been termed exogenous, bottom-up, or stimulus driven the other endogenous, top-down, goal directed. Classically, effects of peripheral cues were called exogenous because they automatically attract attention and are thus effective independently of subjects' intentions. These effects can be called involuntary because they occur even when cues are non-predictive or even counter-predictive of target locations (Posner et al., 1982). Peripheral onset cues have classically been contrasted with endogenous cues that guide attention voluntarily, according to current goals and intentions. These voluntary or endogenous cueing effects are typically thought to occur when cues are informative as to the location of the following target stimulus and subjects make active use of the cues as they are informed about this predictive relationship. Typically, these are studied using central symbolic cues. It has been assumed that endogenous attention and exogenous attention involve different mechanisms (Jonides, 1981; Müller \& Rabbitt, 1989; Prinzmetal, McCool \& Park, 2005), although both can be at work in the same task. Endogenous orienting is thought to require intention and cognitive 
control, as the cue stimulus is shown at a different location from the target and, thus, subjects have to move their attention intentionally to the expected target location, away from the cue stimulus (McCormick, 1997). Intention and cognitive control both have been associated with consciousness (Jack \& Shallice, 2001; Dehaene \& Naccache, 2001).

More recently, involuntary cueing effects have also been reported with other stimuli than peripheral onset cues. Spatially compatible cues such as arrow and eye gaze stimuli (e.g., Friesen \& Kingstone, 1998; Tipples, 2002) or peripheral auditory cues (McDonald, TederSälejärvi \& Hillyard, 2000; Störmer, McDonald \& Hillyard, 2009) can also elicit shifts of spatial attention when they are not predictive of target locations (Ristic \& Kingstone, 2006). The fact that certain types of cues affect attention independently of subjects' intentions and awareness shows that under these circumstances cueing of spatial attention can be characterized as an automatic process.

\subsubsection{Physiological correlates of visual attention}

Physiological methods have been used to study several aspects of the mechanisms of attention. These methods allow conclusions about the locus of attentional selection. Modulation of early visual event related potentials $\mathrm{P} 1$ and $\mathrm{N} 1$ has been interpreted as evidence for early selection, whereas selection at later stages has been identified using other physiological measures (Luck, Woodman \& Vogel, 2000). For instance, in the attentional blink paradigm attention seems to effect post perceptual processes because the N400, which reflects semantic mismatch is unaffected by attention which suggests that words were identified. However, P3 amplitude, which is thought to reflect updating of working memory, was reduced by the attentional blink (Vogel, Luck \& Shapiro, 1998). In the psychological refractory period paradigm, $\mathrm{P} 3$ is affected by attention as well, but in addition attention seems to affect response related processes (Luck, 1998). In visual search experiments, an important 
correlate of spatial attention has been identified and termed N2pc by Luck and Hillyard (1995). These authors presented displays consisting of a target stimulus and several distractors and found a negative shift of potential posterior electrodes contralateral to the location of the target stimulus in the display. This N2pc has been associated with attentional processing of target stimuli that are surrounded by distractors.

In addition, several possible correlates of directing attention have been proposed. Specifically, hemispherical differences have been found between potentials elicited by cues which directed attention to the left and cues which directed attention to the right. Harter, Miller Price, LaLonde, and Keyes (1989) found an increased negativity over posterior electrodes contralateral to the cued side termed early directing attention negativity (EDAN) and a later positive shift over posterior electrodes termed late directing attention positivity or (LDAP). EDAN has been proposed to reflect activity associated with shifts of attention (Harter et al., 1989; Hopf \& Mangun, 2000) but has also been associated with stimulus encoding (Van Velzen \& Eimer, 2003). LDAP has been proposed to reflect a modulation of cortical excitability in preparation of target processing. These correlates of attentional shifts have not yet been studied with unconscious cues and the cue-priming method might provide an opportunity to do that. However, the presence of a separate visible cue stimuli which likely have a larger impact on attention than masked primes makes it difficult to find strong lateralization of priming effects on attention. Applied to the present study, physiological correlates of visual attention could be used to clarify the mechanisms at work in spatial cuepriming. Most promising seem studies of effects on early visual potentials (P1, N1) because these have been used extensively in the study of attention whereas other correlates are comparably poorly understood. 


\subsection{Unconscious cueing of spatial attention}

The question whether unconscious stimuli can direct spatial attention is theoretically meaningful because consciousness has been linked to several executive control functions one of which is the voluntary direction of spatial attention (Baars, 1993). Mulckhuyse \& Theeuwes (2010) reviewed several studies on this topic. However, thus far effects have only been reported for peripheral cues (Kentridge, Heywood, \& Weiskrantz, 1999; Lambert, Naikar, McLachlan, \& Aitken, 1999; McCormick 1997, Mulckhuyse, Talsma \& Theeuwes, 2007, Scharlau \& Ansorge, 2003) and spatially compatible and socially relevant central cues like arrows and eye gaze (Cole \& Kuhn, 2010; Reuss, Pohl, Kiesel \& Kunde, 2011; Sato, Okada \& Toichi, 2007).

\subsubsection{Peripheral cues}

Scharlau and Ansorge (2003) provided evidence that subliminal peripheral cues at the target location can attract spatial attention. In their experiments, metacontrast masked primes were presented before targets in a temporal order judgment task. The authors found that primes which matched the shape of potential targets and were thus consistent with participants' search settings facilitated the perception of targets at the same location relative to perception of targets at other locations; a phenomenon termed "perceptual latency priming". This effect can be explained by the idea that primes with task relevant shapes capture attention like an exogenous cue even when they are masked. Mulckhuyse and colleagues (2007) also found effects of masked peripheral cues that consisted of one of three dots that appeared slightly earlier than the other two in a detection task. They concluded that subliminal sudden onsets can capture attention independently of participants' control settings. These studies suggest that exogenously controlled shifts of attention do not require conscious 
perception of cue stimuli, because peripheral cues can be effective even when participants are unaware of them.

McCormick (1997) reported evidence for a role of awareness in spatial orienting. In his experiment, cues were presented either above or below a subjective threshold of awareness and predicted a target at the opposite side of the screen. With invisible cues participants involuntarily shifted their attention to the location of the dimmed cue. In contrast, when the cue was visible, they were able to use the information conveyed about the likely target location to shift their attention voluntarily away from the cue. The author concluded from this that endogenous orienting requires executive control which depends on the awareness of cue stimuli.

\subsubsection{Central cues}

More recently, three studies reported effects of masked centrally presented cues (Cole \& Kuhn, 2010; Reuss, Pohl et al., 2011; Sato, Okada \& Toichi, 2007). Sato and colleagues (2007) presented masked face stimuli at fixation assuming that gaze direction operates as a spatial cue. Even though cues were not predictive for target locations, participants localized targets more rapidly when they appeared on the side where the gaze cue pointed to than on the opposite side. Cole and Kuhn (2010) found that participants were faster to identify peripheral targets when targets were preceded by a centrally presented masked arrow that pointed to the target location than when the arrow pointed to the opposite location, although arrows were non-predictive for target locations. On the one hand, findings of these two studies with centrally presented cues could be conceived as demonstrations of endogenously governed shifts of attention that are induced by unconscious cues. However, the cueing effects can also be explained without the assumption that endogenous mechanisms are affected by unconscious cues, if one takes into account that these specific stimuli (arrows and eye gaze) 
can affect attention involuntarily (Ristic \& Kingstone, 2006). Therefore, findings could be conceived as instances of involuntary, automatic shifts of attention which can be induced only by spatially compatible cues (Lambert et al., 2006).

Reuss, Pohl and colleagues (2011) proposed that the effect of centrally presented masked cues depends on intentionally set top down influences. Again, arrow stimuli were used as spatial cues for a letter discrimination task. Cues were either valid or invalid indicators of target locations and they were presented masked or unmasked. In contrast to Sato and colleagues (2007) and Cole and Kuhn (2010), Reuss and colleagues reported that masked cues were only effective when they validly predicted the target location whereas visible arrow cues produced validity effects even when they were not predictive for target locations.

\subsubsection{Implicit learning}

In addition, later studies suggest that unseen peripheral cues can direct attention to the opposite side of the cues' location by implicit learning of a predictive relationship between the location of subliminal peripheral cues and target locations (Lambert et al., 1999; Kentridge et al., 1999). Lambert et al. found that participants could learn to use peripheral cues when the cues were predictive for a target on the opposite side of the screen, even though participants were unable to articulate this predictive relationship. Corresponding to authors' implicit peripheral cueing hypothesis, participants responded to the implicitly informative cues only when they were unaware of the relationship and when they were unaware of the cues. These findings contribute to the view that unconscious peripheral cues can modulate visual orientation in a way which goes beyond automatic, exogenously governed shifts of attention to the location of the cue (Lambert et al., 1999). Kentridge and colleagues (1999) found a similar effect in blindsight patient G.Y.. Peripheral cues were presented in G.Y.'s 
blind field which predicted that a target would appear at another location. After some training, G.Y. was able to shift attention away from the cue towards the probable target location. Shifting attention voluntarily away from a cue to the predicted target location cannot be explained by simple mechanisms of automatic shifts of attention to the location of the cue but can be conceived an instance of controlled processing because it requires an interpretation of the meaning and the predictiveness of the cue.

\subsection{Spatial cue-priming}

In summary, it seems as though peripheral cues as well as specific centrally presented cues, like arrows and eye gaze, can elicit shifts of attention even when they are not consciously perceived. Most of these effects are likely based on the mechanisms of involuntary shifts of attention. Effects of predictive peripheral cues, which go beyond simple mechanisms of involuntary shifts of attention (Lambert et al., 1999; Kentridge et al., 1999), depended on effects of implicit learning and it is not entirely clear how these effects relate to the mechanisms of voluntary shifts of attention. However, all of these effects were found with spatially compatible cues that are linked to mechanisms which govern involuntary shifts of attention. To our knowledge, there are currently no reports of corresponding effects of centrally presented symmetrical cues. Therefore, we thought it worthwhile to investigate whether comparable effects with masked centrally presented symmetrical cues could be found which require an interpretation of their meaning. This would provide evidence for the view that the mechanisms which govern endogenous shifts of visual attention are susceptible to the effects of unconscious cues.

The aim of this work was to transfer the cue-priming paradigm to a spatial cueing task to explore possible effects of subliminal symbolic cues on spatial attention. To this end, we combined the cue-priming method developed first by Mattler (2003) with a spatial cueing 
task. Other studies used cue-priming in the context of switching between two tasks (Lau \& Passingham, 2007; Mattler, 2003) or switching attention between modalities (Mattler, 2003). However, the approach can easily be transferred to a spatial cueing task by instructing participants to shift their attention in space according to the cue. In theory, primes should then affect attention shifts if they share critical attributes with the cues.

On the one hand, this approach might be more sensitive to small effects of unconscious cues which potentially enables finding effects of masked symmetrical stimuli; on the other hand, it bears the potential to give further insight in the underlying mechanisms involved in cue-priming because spatial attention has been more intensely studied than other types of attention manipulated in previous cue-priming experiments and thus offers a wide range of experimental paradigms that can be applied to spatial cue-priming. Spatial attention can be used to study the locus of attentional selection leading to the hypothesis that spatial attention is "special" in that it allows for selection at early levels of processing (Hillyard \& Anllo-Vento, 1998). In addition, spatial separation of target stimuli allows indexing the allocation of attention by electrophysiological measures of target processing, i.e., a modulation of target P1 and N1 potentials (Heinze et al., 1990). In an attempt to define the possibility and the basic parameters of spatial cue-priming we conducted the experiments reported in Project 1, in which cue-priming was combined with a letter discrimination task similar to the tasks employed in previous cue-priming studies (Lau \& Passingham, 2007; Mattler, 2003). 


\section{Project 1 - Spatial cue-priming effects in letter discrimination tasks}

The following section 2.1 appeared as 'Masked stimuli modulate endogenous shifts of spatial attention' in 'Consciousness and Cognition' (Palmer \& Mattler, 2013a).

\subsection{Masked stimuli modulate endogenous shifts of spatial attention}

\subsubsection{Abstract}

Unconscious stimuli can influence participants' motor behavior but also more complex mental processes. Recent research has gradually extended the limits of effects of unconscious stimuli. One field of research where such limits have been proposed is spatial cueing, where exogenous automatic shifts of attention have been distinguished from endogenous controlled processes which govern voluntary shifts of attention. Previous evidence suggests unconscious effects on mechanisms of exogenous shifts of attention. Here, we applied a cue-priming paradigm to a spatial cueing task with arbitrary cues by centrally presenting a masked symmetrical prime before every cue stimulus. We found priming effects on response times in target discrimination tasks with the typical dynamic of cue-priming effects (Experiment 1 and 2) indicating that central symmetrical stimuli which have been associated with endogenous orienting can modulate shifts of spatial attention even when they are masked. Prime-cue congruency effects of perceptual dissimilar prime and cue stimuli (Experiment 3) suggest that these effects cannot be entirely reduced to perceptual repetition priming of cue processing. In addition, priming effects did not differ between participants with good and poor prime recognition performance consistent with the view that unconscious stimulus features have access to processes of endogenous shifts of attention. 


\subsubsection{Introduction}

Everyday people are faced with an incoming stream of information about their environment that is too vast to be processed in every detail. Attention can be defined as the mechanism by which people select behaviorally relevant information out of this stream for further processing. Information can be selected according to different features of the input. One mode of attention that has been studied extensively is the deployment of attention across visual space. Frequently, spatial attention is studied in the spatial cueing paradigm (e.g., Posner, 1980). In a typical spatial cueing experiment participants have to detect or identify visual target stimuli that can appear at different locations on a screen. On each trial, a cue stimulus informs participants about the likely location of the subsequent target stimulus (e.g., on the left or right side of the screen). When the target is presented at the predicted location, i.e. the cue is valid, participants show better detection or identification performance than when the cue is invalid and the target is presented at a different location. Cue stimuli can be presented in a variety of ways. Corresponding to the effects of peripherally and centrally presented cues, an early distinction has been made between exogenously and endogenously controlled orienting of attention, respectively (Jonides, 1981; Posner, 1980).

\subsubsection{Endogenously and exogenously controlled shifts of attention}

Effects of peripheral cues have been called "exogenous" because evidence suggested that these cues automatically attract attention rather independently of participants' intentions. Evidence for the involuntary, reflexive nature of these shifts of attention arose from the finding that these effects occur even when cues are non-predictive or even counter-predictive for target locations (Posner, Cohen \& Rafal, 1982). In contrast, effects of centrally presented cues have traditionally been termed "endogenous" because it was assumed that these effects are based on controlled processing which takes current goals and intentions into 
consideration. The "voluntary" nature of these shifts of attention has been derived from the idea that these shifts occur only when cues are informative as to the location of the following target stimulus and participants make active use of the cues because participants are informed about the cues' predictiveness. In the following the term "voluntary" shifts of attention is synonymous to shifts of attention that correspond to the information of predictive cues, whereas "involuntary" shifts of attention refer to shifts that result when cues are nonpredictive.

It has been proposed that exogenous and endogenous shifts of attention result from different mechanisms, although both mechanisms can operate at the same time (Jonides, 1981; Müller \& Rabbitt, 1989; Prinzmetal, McCool, \& Park, 2005). Only endogenous orienting is thought to require intention and cognitive control, because the cue stimulus and the target are shown at different locations and thus, participants have to move their attention intentionally away from the cue to the expected target location (McCormick, 1997). Moreover, intention and cognitive control both have been associated with consciousness (e.g., Dehaene \& Naccache, 2001; Jack \& Shallice, 2001).

Several recent empirical findings, however, have challenged the traditional distinction between non-predictive peripheral cues that induce reflexive, involuntary shifts of attention due to automatic, exogenously triggered processes on the one hand, and predictive centrally presented cues that induce intentional, voluntary shifts of attention due to controlled, endogenously governed processes on the other hand (e.g., Kingstone, Smilek, Ristic, Friesen \& Eastwood, 2003). In an fMRI study Peelen, Heslenfeld and Theeuwes (2004) found that shifts of attention to central and peripheral cues recruit similar neural mechanisms. The distinction is also questioned by validity effects of peripheral cues on shifts of spatial attention. For instance, Posner and colleagues (1982) used peripheral cues that were 
predictive for a target at another location and found that participants were able to use the information conveyed about the likely target location to shift their attention voluntarily away from the cue. On the other hand special types of centrally presented cues that lack any predictive value can also trigger shifts of attention rather automatically. For instance, spatially compatible cues such as centrally presented arrows or eye gaze stimuli (e.g., Friesen \& Kingstone, 1998; Tipples, 2002) can elicit shifts of spatial attention even when the cues are not predictive for target locations (Ristic \& Kingstone, 2006). These effects suggest that shifts of spatial attention in response to these centrally presented cues are also susceptible to overlearned, involuntary, automatic processes. The assumption that orienting to centrally presented arrow cues is an endogenous voluntary process is further questioned by a recent study by Risko and Stolz (2010) in which implicit learning seemed to play an important role in the orienting of attention to the cues, rather than participants' insights into the validity of cues in the current context.

Lambert, Roser, Wells and Heffer (2006) have recently suggested that a distinction should be made regarding whether central or peripheral stimuli that serve as cues enable spatial correspondence learning rather than assuming different mechanisms to account for spatial cueing effects with central and peripheral cues. These authors assume that it is important whether stimuli enable an associative learning of spatial correspondences between the cue display and the target display. Spatial correspondence can be learned rather easily with asymmetrical central and peripheral cues. A weak form of spatial correspondence learning can also occur with bilateral symmetrical stimuli like two Ts and two Xs that are concurrently presented left and right of fixation (e.g., Lambert \& Duddy, 2002; Shin, Marrett \& Lambert, 2011). In contrast, however, when a single symmetrical stimulus like $\mathrm{T}$ or $\mathrm{X}$ serves as a predictive cue, spatial correspondence learning should not occur. This view has 
been supported by the absence of spatial cueing effects with these stimuli (Lambert et al., 2006; Shin et al., 2011). In sum, these challenging findings call for an elaboration of the characteristics of the processes that are engaged in endogenous and exogenous shifts of attention. This should also incorporate the role of cue stimuli that enable spatial correspondence learning and symmetrical stimuli that do not, which might correspond to exogenously and endogenously governed shifts of attention, respectively. In the present study we used centrally presented symmetrical cue stimuli to examine endogenous shifts of spatial attention and the issue whether such shifts of attention depend on visual awareness of the effective features of cue stimuli.

\subsubsection{The effects of invisible cues on shifts of attention}

Attention and consciousness are somewhat related, yet distinct concepts (Koch \& Tsuchiya, 2007). Several studies have attempted to investigate effects of unconscious cues on shifts of spatial attention. To this end, cue stimuli were either reduced in size or contrast or followed by masking stimuli in order to reduce their visibility. A series of studies suggests that spatially compatible cues can affect shifts of spatial attention even when the effective cues are masked in a way that participants are not aware of them. This has been reported for peripheral (Kentridge, Heywood, \& Weiskrantz, 1999; Lambert, Naikar, McLachlan, \& Aitken, 1999; McCormick 1997, Mulckhuyse, Talsma \& Theeuwes, 2007, Scharlau \& Ansorge, 2003) and central cues (Cole \& Kuhn, 2010; Reuss, Pohl, Kiesel \& Kunde, 2011; Sato, Okada \& Toichi, 2007). For a review see Mulckhuyse \& Theeuwes (2010).

Of particular relevance is the study by McCormick (1997), who contrasted the effects of predictive and non-predictive peripheral cues. Cues predicted a target at the opposite side of the screen. Stimulus contrast of the cues was varied so that they were either above or below a subjective threshold of awareness. Although participants reported that they had not seen the 
cues, McCormick (1997) found involuntary shifts of attention to the location of the lowcontrast cue. In contrast, when participants reported to see the cue they were able to use the information conveyed about the likely target location to shift their attention voluntarily away from the cue. The author concluded from this that endogenous orienting requires executive control which depends on the awareness of cue stimuli.

All of the studies on effects of invisible cues on spatial attention mentioned above used spatially compatible stimuli that can be linked to mechanisms which govern involuntary shifts of attention. To our knowledge, there are currently no reports of corresponding effects of centrally presented symmetrical cues. Therefore, we considered it worthwhile to investigate whether comparable effects with masked centrally presented symmetrical cues could be found. Central symmetrical cues are special in that they do not enable spatial correspondence learning (Shin et al., 2011) and they differ from arrows and eye gaze stimuli because they have no overlearned associations to a spatial direction. Therefore, centrally presented symmetrical cues require an interpretation of their meaning which has been associated to endogenous control of spatial attention. We reasoned that an effect of unconscious stimulus features of central symmetrical stimuli on shifts of spatial attention could be taken as direct evidence for the view that the mechanisms which govern endogenous shifts of visual attention are susceptible to the effects of unconscious information.

\subsubsection{The cue-priming paradigm}

The studies mentioned above potentially challenge the traditional distinctions between involuntary, exogenous shifts of attention that are triggered by peripheral cues and voluntary, endogenous shifts caused by central cues and also challenge the corresponding links to automatic and controlled mechanisms that are influenced by unconscious and conscious stimuli, respectively. Presently, however, there is no convincing evidence for effects of 
unconscious stimuli on mechanisms of endogenous shifts of attention. To the extent that endogenous shifts of attention are governed by executive control processes, it could be assumed that these control processes require consciously accessible stimuli. Recently, however, this notion has been called into question both theoretically (Hommel, 2007) and empirically in studies that showed effects of unconscious stimuli on processes of task preparation (Lau \& Passingham, 2007; Mattler, 2003b, 2005, 2007) and response inhibition (Krüger, Klapötke, Bode \& Mattler, 2012; van Gaal, Ridderinkhof, Fahrenfort, Scholte \& Lamme, 2008; van Gaal, Ridderinkhof, van den Wildenberg, \& Lamme, 2009).

For instance, Mattler (2003b, 2005) showed priming effects of metacontrast-masked stimuli in several cueing tasks thought to involve executive control processes. In Experiment 3 of his study participants were instructed to shift their attention to either the auditory or visual modality according to a symbolic cue stimulus (a square or a diamond). Unbeknownst to them, this cue stimulus was always preceded by a masked prime which was also either a square or a diamond. When the cue and the prime had the same shape, participants were faster to identify a target stimulus in the cued modality than when they were of different shapes. This was interpreted as evidence that unconscious stimulus features can affect shifts of attention between modalities. On the one hand, spatial attention has been distinguished from other kinds of attention (Hillyard \& Anllo-Vento, 1998) consistent with the view that space is a "special" stimulus dimension for the control of visual attention (e.g., Treisman \& Gelade, 1980; Treisman, 1998). On the other hand, recent evidence suggests that orienting of spatial attention shares some mechanisms with other kinds of attentional preparation similar to those examined in previous cue-priming studies (Chiu \& Yantis, 2009; Slagter, Kok, Mol, \& Kenemans, 2005). Therefore, we thought it interesting to transfer Mattler's cue-priming paradigm to a spatial cueing task. Given that previous cue-priming studies mostly used central 
symmetrical cues, such a transfer might help to clarify the issue whether unconscious features of centrally presented symmetrical stimuli can affect spatial attention.

The present study was designed to employ a cue-priming paradigm in the domain of spatial attention. To the extent that performance in a spatial attention task is affected by unconscious cue-priming in the same way as in other tasks, priming effects should exhibit similar time courses when stimulus onset asynchronies (SOA) between the critical stimuli are varied. This could increase the amount of evidence for the view that cue-priming is a domain independent phenomenon. Beyond this, we aimed to employ stricter objective measures of prime visibility than previous studies that investigated effects of masked central cues (Cole \& Kuhn, 2010; Sato et al., 2007). In addition, we thought it is important to control for eye movements to rule out the possibility that effects result from priming of oculomotor processes. The premotor theory of attention (Rizzolatti, 1987) proposes that spatial attention overlaps with the programming of saccades. If spatial attention is indeed based on subthreshold saccade activation, cue-priming effects in a spatial cueing task could possibly be explained solely on a motor level. In this case priming of spatial attention could be more similar to motor than to non-motor priming (Mattler, 2003b). To control for such motor effects we tracked eye movements in all experiments and excluded all trials on which eye movements occurred.

\subsubsection{Experiment 1}

In Experiment 1 we transferred the cue-priming paradigm to a spatial cueing task. Based on previous cue-priming effects we predicted that similar effects should emerge in the spatial task with faster responses when prime and cue are congruent than when they are incongruent. Corresponding to previous cue-priming experiments we varied cue-target SOA 
and expected that cue-priming effects decrease with increasing cue-target SOA (Mattler, 2005).

\subsubsection{Method}

Participants. 12 participants took part in 3 sessions of the experiment in exchange for course credit. Four additional participants were excluded from the experiment because of a large amount of eye movements, problems with pupil detection or a misunderstanding of the task. Of the remaining 12 participants (age 21-32, $\mathrm{M}=23.6$ ), 5 were male and 7 female.

Task. The task we used here was similar to the tasks Mattler (2003b) used, while incorporating spatial location as the relevant information conveyed by the cue. Participants had to shift their attention on the screen either to the left or to the right depending on a cue, which was either a square or a diamond shaped stimulus. Participants were instructed to rest their head in a chinrest and hold still during blocks.

Apparatus. Stimuli were displayed on a Viewsonic 19"' Perfect Flat monitor using a resolution of $1024 * 768$ pixels and $100 \mathrm{~Hz}$ refresh rate. The experiment was controlled by Presentation software (Neurobehavioral Systems, www.neurobs.com). The monitor was placed in $67 \mathrm{~cm}$ viewing distance. Eye movements were recorded with an Eyelink 1000 eyetracker (SR-Research, binocular mode, $1000 \mathrm{~Hz}$ temporal resolution). Synchronization of the experiment software and the eye tracking device was realized using "The Presentation Extension for the EyeLink Eyetracking System" (Prexel, http://www.cs.umb.edu/ marc/prexel/). Calibration of the eye tracking device was done before the experiments and in breaks if necessary. 


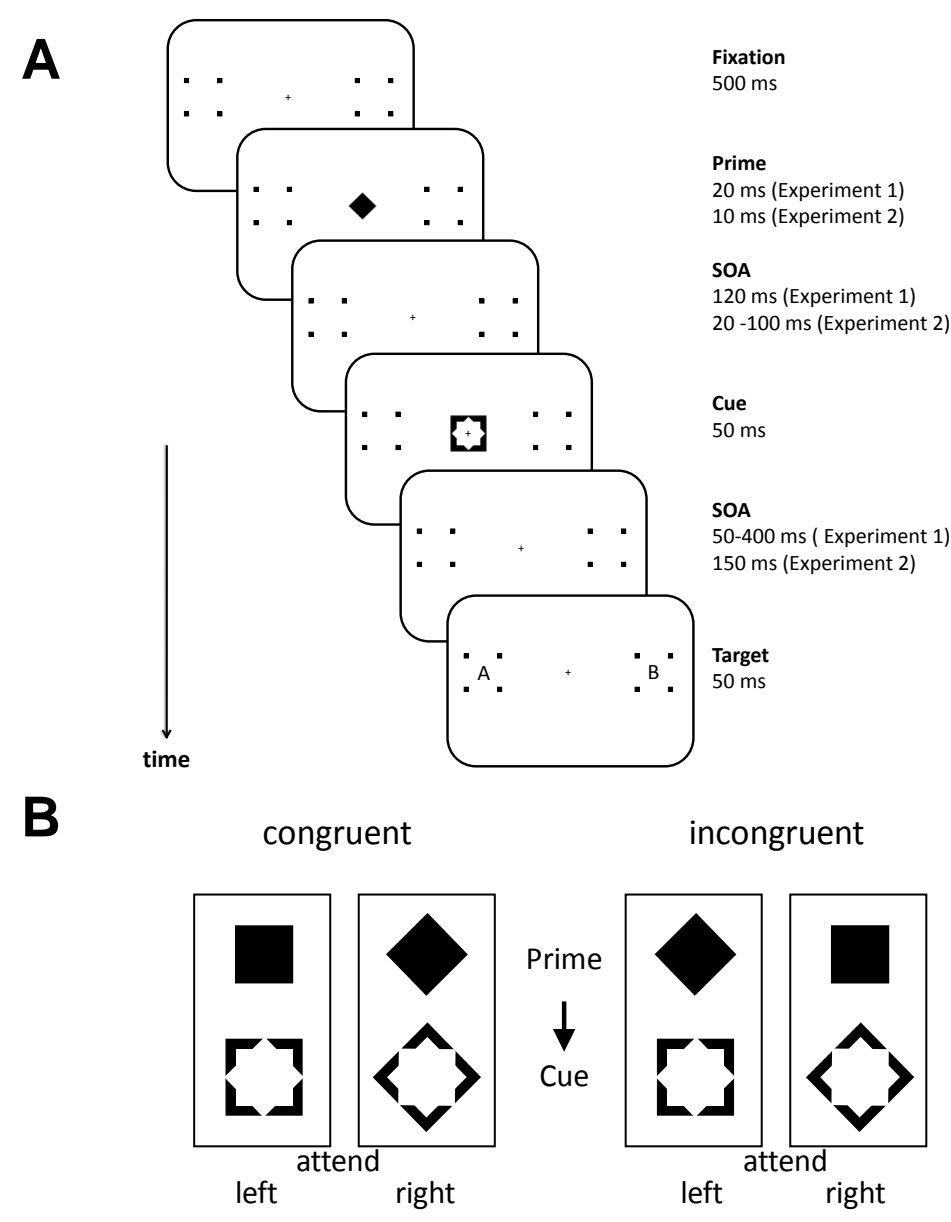

Figure 2.1 Stimuli in Experiments 1 and 2. (A) Sequence of events in a trial. Experiments 1 and 2 differed in timing of events. See text for further description. (B) Possible combinations of prime and cue stimuli and assignment to directions for square-left diamond-right participants.

Stimuli. Stimuli were presented in black, fixation and target markers in red. The cue was preceded by a prime stimulus that was also either a square or diamond (see Figure 2.1A). Participants were not told about the presence of the primes until after the experiment. Prime and cue could either have the same outer shape (congruent) or different shapes (incongruent). The cues also served as metacontrast masks for the primes. This was possible because cues had cutouts that fit right around the primes (with one line of pixels in between). See Figure 2.1B for a depiction of cue and prime stimuli. Subsequent to the cue two letters were presented ( $\mathrm{A}$ and $\mathrm{B}$, one on each side of the screen) and participants had to indicate whether 
the target letter on the cued side was an A or B by pressing one of two keys ('+' and 'Enter' on the keyboard number pad). Thus, cues in this task were $100 \%$ valid and had to be used to find the correct response, as the letters on both sides always differed. Participants used index fingers of both hands, one for each key. Participants were instructed to keep fixation on the red cross in the center of the screen at all times and to blink only between trials. It was emphasized that they should use the cue to actively shift their attention as fast as possible.

The onset of a trial was marked by the appearance of 4 red dots on each side of the screen that marked the potential target locations and vanished again after each response. After $500 \mathrm{~ms}$ a prime was presented for $20 \mathrm{~ms}$ and a cue followed with prime-cue SOA of $120 \mathrm{~ms}$. Cues and targets were present for $50 \mathrm{~ms}$ each. We varied cue-target SOA between blocks in 5 steps $(50,100,150,200$ and $400 \mathrm{~ms})$. After target offset, participants had 2 seconds to respond. Assignment of cues to sides and letters to keys was balanced across participants. Central stimuli had side lengths of $2.7^{\circ}$ (cues) and $1.7^{\circ}$ (primes) visual angle. Target letters A or B subtended $1.3^{\circ} \times 1.1^{\circ}$ visual angle. They were located $8^{\circ}$ visual angle left and right to the centre of the screen. Their locations were marked by four dots on each side that were arranged to form a square with a side length of $3^{\circ}$ visual angle. After each trial in which participants chose the wrong response or did not respond in time error feedback was given in form of a beep tone $(1000 \mathrm{~Hz}, 300 \mathrm{~ms})$. This tone delayed the start of a new trial by $300 \mathrm{~ms}$. To give participants immediate feedback about their eye-movements, we employed a method devised by Guzman-Martinez, Leung, Franconeri, Grabowecky \& Suzuki (2009). In this procedure participants are exposed to two complementary random dot patterns made of $50 \%$ black and $50 \%$ white pixels. Dots that are white in one pattern are black in the following pattern and vice versa. When these two patterns follow one another in rapid alternation participants perceive a grey background if they do not move their eyes because the black-and-white dots 
follow each other at the same location and average out. Whenever participants move their eyes, however, this averaging is interrupted and the black and white pattern is perceived as a zigzag pattern. Thus, whenever participants see the zigzag pattern they know that they had moved their eyes. We used such an alternating background of two complementary black and white random dot patterns with each dot measuring 4 x 4 pixels in size that flickered with 50 Hz. This background covered the entire screen and all other stimuli were presented on top of it. The fixation cross and alternating background were present at all times. We explained this procedure before the experiment and presented a short example asking participants to move their eyes while looking at the alternating background.

Design and procedure. Participants completed three sessions, each with a short training phase at the beginning and 640 experimental trials. The first session was designed as a practice session, starting with 320 trials with a cue-target SOA of $400 \mathrm{~ms}$ and ending with 320 trials with a cue-target SOA of $150 \mathrm{~ms}$. Data from this session were not included in the analyses. The other two sessions consisted of 5 blocks of 128 trials, each with one of the five SOAs. In each block each combination of 2 primes, 2 cues, and 2 target arrays was presented 16 times resulting from the two sessions in a total of 128 congruently primed and 128 incongruently primed trials at each level of cue-target SOA, pooling across the two cues and the two target arrays. The order of these blocks was randomized in each session. After every 32 trials there was a break in which participants could rest and abstain from keeping fixation for as long as they wished. They ended the break by pressing the space bar.

Analyses. Trials on which eye movements occurred were excluded from analyses to ensure that effects of the primes were due to spatial attention and not due to eye movements. For each trial we computed a baseline position for each eye averaging the position over $100 \mathrm{~ms}$ (100 samples) before prime onset. Participants were instructed to keep their eyes on the 
fixation cross at the center of the screen during this and the following period of time. Relative to this baseline we computed shifts in gaze position in the time window from prime onset to target offset. Trials on which relative gaze position deviated from the baseline position more than $0.56^{\circ}$ of visual angle were excluded from the analyses of response times and error rates. Every trial that had at least one sample in which eye position data was missing (blink) in the critical time period from prime onset to target offset was excluded as well. Note that the critical time period increases with increasing prime target SOA. Therefore, the probability of eye movement errors increases with SOA, due to the increasing length of the critical period and a correspondingly increasing probability of drifts away from baseline position.

Reaction times (RTs) were averaged for correct responses excluding post error trials and analyzed with a repeated-measures ANOVA with factors Prime-Cue Congruency and Cue-Target SOA. Rate of eye movement errors (including blinks) and rate of response errors in all trials were arc-sine transformed and analyzed with a repeated-measures ANOVA. Reported p-values are Greenhouse-Geisser corrected where appropriate.

\subsubsection{Results}

Reaction times in Experiment 1 are shown in Figure 2. Reactions were faster on congruent trials $(629 \mathrm{~ms})$ than on incongruent trials $(677 \mathrm{~ms})$ as revealed by a main effect of Prime-Cue Congruency, $F(1,11)=52.6, M S e=1268, p<.001$. RTs decreased with increasing Cue-Target SOA from $745 \mathrm{~ms}$ to $537 \mathrm{~ms}$, resulting in a significant main Effect of SOA, $F(4,44)=119.8, M S e=1362, p<.001$. There was also a significant interaction between these two factors, $F(4,44)=4.8, M S e=536, p=.002$, indicating that the priming effect decreased with SOA (from $61 \mathrm{~ms}$ to $10 \mathrm{~ms}$ ). 

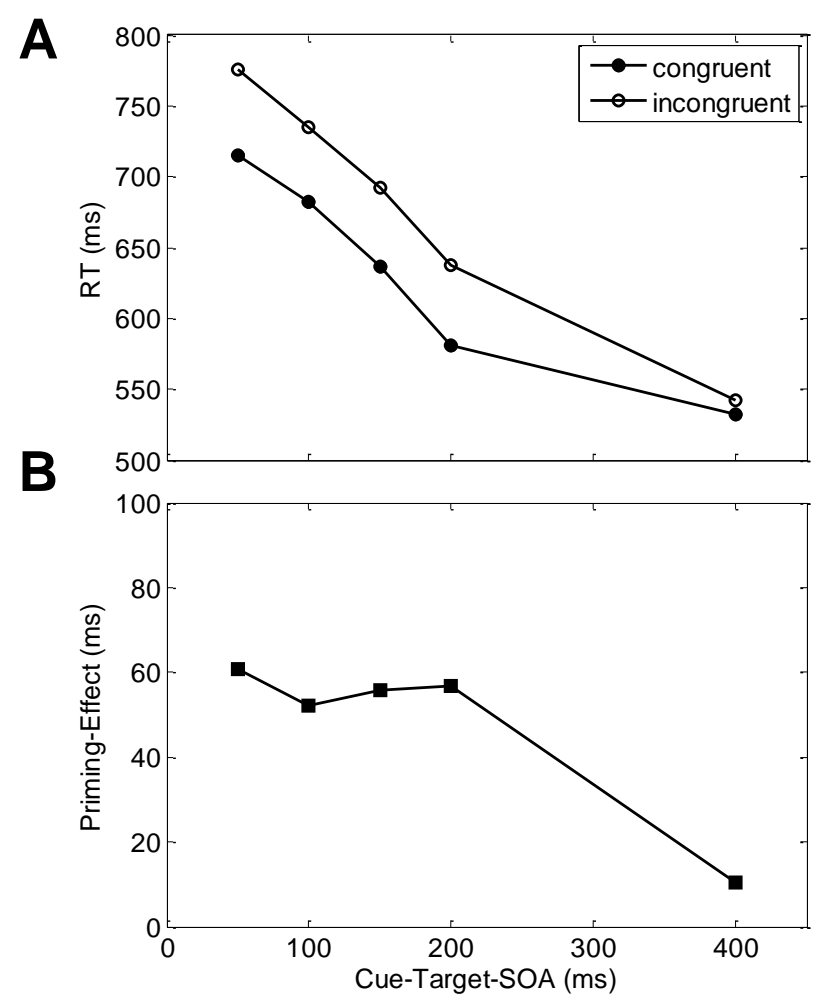

Figure 2.2 Results in Experiment 1. (A) RT for congruent and incongruent trials as a function of cue-target SOA. (B) Priming effect on RT (incongruent-congruent) as a function of cue-target SOA.

Table 2.1 shows rates of response errors and eye movement errors in Experiment 1. As expected, there was a main effect of SOA on eye movement error rates which increased from $3.9 \%$ to $17.8 \%, F(4,44)=28.9, M S e=0.029, p<.001$. The interaction between SOA and Prime-Cue Congruency on eye movement errors did not reach significance, $F(4,44)=2.5$, $M S e=0.009, p=.091$. Neither the main effect of Prime-Cue Congruency $(F<1, p>.8)$ nor the main effect of $\operatorname{SOA}(F(4,44)=2.0, M S e=0.012, p=.106)$ was significant.

The analysis of response errors, however, revealed a significant interaction between SOA and Prime-Cue Congruency, $F(4,44)=5.4, M S e=0.009, p=.001$. In order to further evaluate this interaction we performed paired t-tests comparing error-rates between congruent and incongruent trials at each SOA. These tests revealed significant prime-cue congruency 
Table 2.1 Error rates and excluded trials in Experiment 1

\begin{tabular}{|c|c|c|c|c|c|c|c|c|c|c|}
\hline \multirow{3}{*}{$\begin{array}{l}\text { Prime-Cue } \\
\text { Congruency }\end{array}$} & \multicolumn{10}{|c|}{ Cue-Target SOA(ms) } \\
\hline & 50 & 100 & 150 & 200 & 400 & 50 & 100 & 150 & 200 & 400 \\
\hline & \multicolumn{5}{|c|}{ Error Rate $(\%)$} & \multicolumn{5}{|c|}{ Trials Excluded (\%) } \\
\hline Congruent & 4.4 & 2.7 & 3.9 & 2.0 & 4.7 & 3.5 & 6.5 & 6.1 & 8.3 & 19.4 \\
\hline Incongruent & 4.5 & 3.1 & 3.3 & 3.9 & 3.6 & 4.3 & 5.5 & 6.4 & 9.2 & 16.2 \\
\hline
\end{tabular}

effects at the two longest SOAs. With $200 \mathrm{~ms}$ SOA participants committed more errors on congruent trials than on incongruent trials, $2.0 \%$ vs. $3.9 \%$, respectively, $t(11)=-3.3, p=.007$, whereas the opposite was true with $400 \mathrm{~ms} \mathrm{SOA}, 4.7 \%$ and $3.6 \%, t(11)=2.6, p=.025$.

\subsubsection{Discussion}

We found an effect of masked central symbolic primes on reaction times in a spatial cueing task. This effect decreased with increasing cue-target SOA. This time course resembles the time courses Mattler (2005) found for cue-priming in other tasks. He obtained priming effects that were short-lived and decayed almost completely at about $400 \mathrm{~ms}$ after cue presentation. This similarity might hint at similar underlying mechanisms. The time course of cue-priming effects contrasts, however, to effects of cue validity which have been found to increase with cue-target SOA. This may be explained by assuming that the relative contribution of primes and cues to the orienting of attention changes with SOA in such a way that the effect of cues increases with longer SOAs whereas the effects of primes might decrease (see General Discussion). Error rates, which were small in general, showed a primecue congruency effect with $200 \mathrm{~ms}$ SOA that surprisingly reversed with $400 \mathrm{~ms}$ SOA. 
Possibly this finding might be related to inhibitory effects that reverse the effect of the prime after a certain time, e.g., negative compatibility effect (Eimer \& Schlaghecken, 1998) or inhibition of return (Posner \& Cohen, 1984).

The results of Experiment 1 suggest that it is possible to extend cue-priming (Mattler, $2003 b$ ) to a spatial cueing paradigm. However masking of the primes was rather weak, as we employed conditions likely to maximize priming effects and not minimize prime visibility. This was evidenced by participants' subjective reports after the experiment. 10 of 12 participants reported having seen the primes on every trial. Consequently, we did not collect objective measures of prime visibility. The fact that participants saw the prime does not mean that prime visibility is a necessary condition for priming effects. To address this issue we conducted Experiment 2, which also aimed at examining the role of prime-cue SOA on priming effects in this task.

\subsubsection{Experiment 2}

Experiment 2 aimed at investigating the effect of prime-cue SOA on spatial cuepriming. As Experiment 1 showed a similar effect of cue-target SOA for spatial cue-priming as cue-priming effects in other tasks (Mattler, 2005), we expected that varying prime-cue SOA would also yield similar results as in other tasks. Previous research showed that cuepriming effects increase almost monotonically with increasing prime-cue SOA up to $119 \mathrm{~ms}$ (Mattler, 2003b). Therefore we expected responses to be faster on congruent than on incongruent trials and that this priming-effect increases with increasing prime-cue SOA. 


\subsubsection{Method}

Participants. We recruited 12 new participants from the University of Goettingen. One additional participant was excluded due to excessive blinking. Of the remaining 12 participants (age 21-28, M =24.3), 4 were female and 8 were male.

Task. In the priming sessions, participants had the same task as in Experiment 1. We used the same mapping of cues to sides and letters to keys for all participants. In the final prime recognition session, participants were asked to indicate whether the prime stimulus was a square or a diamond using the same two keys as in the task before. We used a prime recognition task rather than a prime detection task because we were most interested in the issue whether participants can discriminate the stimulus features that are crucial for the priming effect (Schmidt \& Vorberg, 2006). Participants were encouraged to take time and had no time limit for their responses. Auditory error feedback was given on each erroneous trial in both tasks.

Stimuli. Stimuli and setup were the same as in Experiment 1 (see Figure 2.1) with the following exceptions. The onset of a new trial was not marked by the location markers but by the fixation dot turning into a cross which remained on the screen until a response was given. Location markers remained on the screen during the entire block of trials. In contrast to Experiment 1 fixation and target location markers were black. In Experiment 2 we varied prime-cue SOA within blocks in 5 steps of $20 \mathrm{~ms}$ from $20 \mathrm{~ms}$ to $100 \mathrm{~ms}$. We used a constant cue-target SOA of $150 \mathrm{~ms}$, because we wanted that our participants were able to prepare for the target, which some participants found difficult at the short SOAs in Experiment 1. In addition we decreased prime duration to $10 \mathrm{~ms}$ to achieve better masking and measured prime visibility in a separate session. The shifting random dot background was only present in the 
practice session, because it partially overwrites stimuli with duration of only one frame which we used for our primes.

Design and procedure. Participants completed three sessions of the choice-RT task. The first session was only for practice purposes. In this session, cue duration and cue-target SOA were longer than in later sessions and the data were not used in any analyses. The second and third session each included 640 trials 40 of which were randomly inserted no-go trials to prevent use of the strategy to only attend to one side of the screen and the cue without shifting attention. On these no-go trials, a $\mathrm{Z}$ was presented on the cued side and participants were to withhold their response. Prime-cue SOA was varied within blocks instead of between blocks. Each combination of 2 primes, 2 cues, 5 SOAs and 2 target arrays was presented 15 times in each session, resulting in a total of 120 congruent and 120 incongruent trials at each SOA, pooling across the two sessions, the two cues and the two target arrays. On no-go trials each combination was presented once in either session. There was a break every forty trials. In addition, we tested prime visibility in a separate fourth session with 128 trials at each level of SOA.

Apparatus. We used the same setup as in Experiment 1.

Analyses. RT error data and eye movements were computed and analyzed in the same way as in Experiment 1. Prime recognition performance was analyzed using signal detection measures. We computed d' separately for each mask (square and diamond) with each SOA. These values were then averaged across masks and analyzed with a repeated-measures ANOVA and t-tests against zero. 


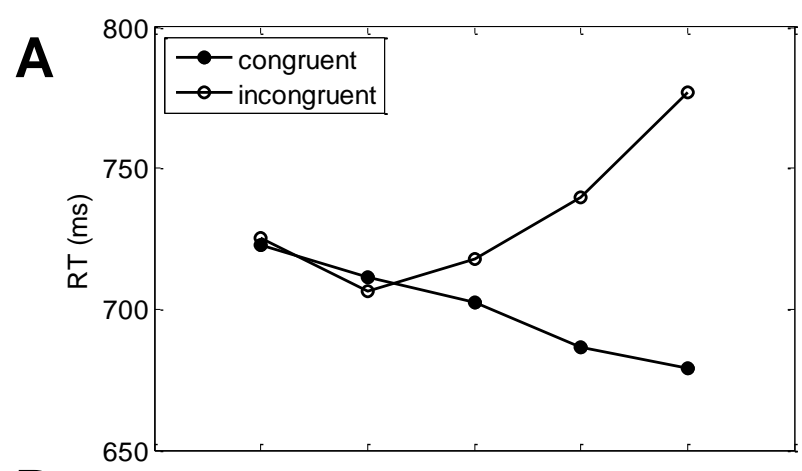

B

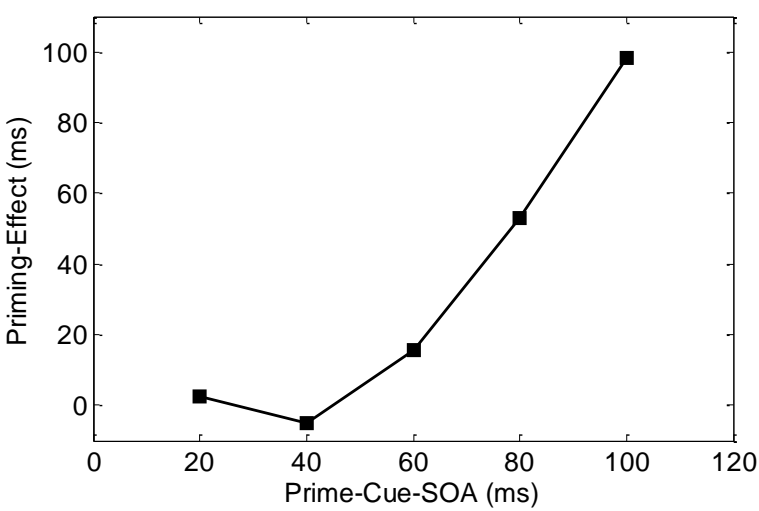

Figure 2.3 Results in Experiment 2. (A) RT for congruent and incongruent trials as a function of prime-cue SOA. (B) Priming effect on RT (incongruent-congruent) as a function of prime-cue SOA.

\subsubsection{Results}

Figure 2.3 shows reaction times and the priming effect in Experiment 2. A repeatedmeasures ANOVA of reaction times revealed a significant main effect of Prime-Cue Congruency, $F(1,11)=6.1, M S e=5325, p=.031$. Participants responded faster on congruent trials $(701 \mathrm{~ms})$ than on incongruent trials $(733 \mathrm{~ms})$. This effect was influenced by prime-cue SOA as priming effects increased from $2 \mathrm{~ms}$ at $20 \mathrm{~ms}$ SOA to $98 \mathrm{~ms}$ at $100 \mathrm{~ms}$ SOA. This resulted in a significant interaction between Prime-Cue Congruency and SOA, $F(4,44)=$ 11.3, $M S e=967, p<.001$.

Rates of response and eye movement errors in Experiment 2 are compiled in Table 2.2. Due to large inter-individual differences, neither factor had an effect on response error 
Table 2.2 Error rates and excluded trials in Experiment 2

Prime-Cue SOA(ms)

\begin{tabular}{|c|c|c|c|c|c|c|c|c|c|c|}
\hline \multirow[t]{2}{*}{$\begin{array}{l}\text { Prime-Cue } \\
\text { Congruency }\end{array}$} & 20 & 40 & 60 & 80 & 100 & 20 & 40 & 60 & 80 & 100 \\
\hline & \multicolumn{5}{|c|}{ Error Rate (\%) } & \multicolumn{5}{|c|}{ Trials Excluded (\%) } \\
\hline Congruent & 4.2 & 3.2 & 3.6 & 4.0 & 2.9 & 4.5 & 4.5 & 4.5 & 5.2 & 5.1 \\
\hline Incongruent & 4.4 & 3.5 & 3.6 & 3.8 & 5.5 & 2.7 & 3.8 & 4.4 & 4.0 & 4.3 \\
\hline
\end{tabular}

rates $(p>.4$ in all cases) or eye movement errors ( $p>.1$ in all cases). Across all 80 no-gotrials no participant made more than 3 errors resulting in a mean error rate of $1.4 \%$.

Prime visibility. When asked after the final priming session, five participants reported not having noticed primes at all. The remaining participants reported to have seen the presence of the primes in at least 20 per cent of trials. Prime recognition performance as measured in the fourth session increased with SOA as evidenced by a main effect of SOA, $F(4,44)=4.7, M S e$ $=0.44, p=.003$, with $\mathrm{d}^{\prime}=0.7$ with $20 \mathrm{~ms}$ SOA, 0.8 with $40 \mathrm{~ms}$ SOA, 1.2 with $60 \mathrm{~ms}$ SOA, 1.4 with $80 \mathrm{~ms} \mathrm{SOA}$, and 1.7 with $100 \mathrm{~ms}$ SOA. t-tests on d' against zero revealed that participants performed better than chance at all SOAs: $t>2.7, p<.02$ in all cases.

\subsubsection{Discussion}

We found evidence of priming effects of masked stimuli on shifts of spatial attention in a spatial cueing task. Results show that the masking procedure hindered prime visibility and therefore suggest that shifts of spatial attention are modulated by stimuli that are difficult to perceive. Unfortunately, the masking procedure that we used was not as effective as we expected it to be, and a majority of participants reported that they detected the presence of the 
primes during the priming sessions. Participants performed above chance levels in the following prime recognition session indicating that they could discriminate the effective stimulus features under these conditions. Thus, this objective measure of prime discrimination performance obtained in the recognition session seems to be more conservative than the subjective measure obtained by questioning participants after the prime recognition session. Our subjective measure of prime visibility is probably affected by the fact that participants allocated attention to primes in the prime recognition session but not during the priming sessions. Considering that objective measures of prime visibility are the standard in masked priming research, relying on these subjective reports to draw conclusions about the invisibility of primes would be overly lenient. Therefore, the data of Experiment 2 are equivocal regarding the issue whether unconscious stimulus features would be sufficient to affect spatial shifts of attention. Indirect evidence for the latter view is given by the time course of the priming effects in Experiment 2 because it corresponds to the time course of other cue-priming effects which have been shown to be independent of prime visibility (Mattler, 2003b).

We think the cue-priming procedure might be especially sensitive to effects of masked stimuli on attention shifts due to the use of a clearly visible cue stimulus that has to be processed to do the task. This paradigm has the potential to find effects of primes that are too weak to initiate full shifts of attention by themselves, but nonetheless affect shifts triggered by the cue. On the other hand, however, cue-priming effects have to be interpreted carefully because there are other mechanisms conceivable that could explain how primes affect response times without actually affecting task selection or allocation of attention in the present paradigm. As mentioned by Mattler (2003b) measures of response time in simple cuepriming paradigms like the preceding experiments of this study include components of cue 
identification, task selection, and task execution. Therefore, critics like Dehaene (2008) proposed that "the observed priming might have arisen from the perceptual component alone" (p. 33), with congruent primes leading to faster response times due to perceptual priming and incongruent primes leading to slower response times due to perceptual conflict. Evidence for a perceptual component has been reported by Mattler (2006) who found that responses were faster when cues were preceded by perceptually similar primes than by perceptually dissimilar primes whose shapes were nonetheless associated to the same task as the cue. However, perceptually dissimilar primes still showed significant congruency effects, because response times were faster when the shape of the primes was congruent and dissimilar to the cue than when primes were incongruent. This has been taken as evidence for the view that part of the cue-priming effects result from post-perceptual stages of processing. Results of Lau \& Passingham (2007) support this view and suggest that masked primes can indeed affect task selection. These authors adopted Mattler's cue-priming task in an fMRI setting, to show that primes modulate neural activity in brain areas associated with the respective task. Most importantly, Lau and Passingham found that cortical brain areas can be activated by incongruent primes even when the visible cue activates different brain areas that are relevant for the task associated to the cue. These findings suggest that cue-priming effects are not entirely based on perceptual interactions between prime and cue processing. In line with this view, Reuss, Kiesel, Kunde \& Hommel (2011) found effects of masked cue stimuli on task choices when masked cues were presented alone without a separate visible cue. However, due to the fact that primes and cues in Experiments 1 and 2 of the present study had similar shapes, these spatial cue-priming effects might be reduced to the perceptual interaction between primes and cues. To clarify whether later levels of processing contribute to the present cue-priming effects, we conducted a third experiment. 


\subsubsection{Experiment 3}

The purpose of Experiment 3 was twofold. On the one hand, we tried to find evidence for the view that the cue-priming effects on shifts of spatial attention are independent of prime visibility. In addition we tried to localize the source of the cue-priming effects on shifts of spatial attention by using perceptually similar and dissimilar prime-cue pairs to contribute to the issue whether primes do indeed affect shifts of spatial attention rather than simply facilitate or inhibit the perceptual processing of the subsequent cue stimuli. Following the logic of Mattler's (2006) experiments, we attempted to disentangle perceptual and postperceptual sources of cue-priming effects by mapping two cues to each direction of attention. We reasoned that, if primes exert influence only on a perceptual level, cue-priming effects should be absent when primes and cues have different shapes. However, if there is a postperceptual component in cue-priming of spatial attention perceptually dissimilar but congruent prime-cue pairs should lead to faster RTs than perceptually dissimilar-incongruent prime-cue pairs.

\subsubsection{Method}

Participants. 16 new participants were examined in 5 sessions of Experiment 3. Seven additional participants were excluded after the first session because of failure to comply with task instructions or problems with eye gaze detection. One additional participant was excluded because this participant consistently showed negative d' values in the prime recognition task. Of the remaining participants (age 20-32, mean 22.3), 4 were male and 12 female.

Tasks. In the choice-RT sessions the task was the same as in Experiment 2, except that this time two different symbols were associated with each side. In the prime recognition session, participants were asked to identify the prime using the same two response keys that they used 
before. We used this mapping of two symbols to one key to make the prime recognition task as similar as possible to the choice-RT task (Schmidt \& Vorberg, 2006). Symbols that were mapped to a shift of attention to the left in the priming session were mapped to the upper response key (“"+” on the number pad) while the other two symbols were mapped to the lower key ("Enter" on the number pad). Participants were instructed to respond as accurately as possible and to guess whenever they were unsure. Again, feedback was given after each erroneous response.

Apparatus. The same setup as in the previous experiments was used again. In Experiment 3 we used a refresh rate of $85 \mathrm{~Hz}$.

Stimuli. Figure 2.4 gives an overview of the stimulation in Experiment 3. Stimuli were variations of the primes and cues used in Experiments 1 and 2. They were made by cutting off the top or bottom of the diamond stimuli and adding the respective parts to square stimuli, resulting in four different symbols. Thus, primes still fitted inside the cut-outs in the cue stimuli. Symbols with the top changed signaled a target on the left side, whereas those with the bottom part changed signaled a target on the right side. Instead of only four different combinations of prime and cue there were sixteen different combinations which can be ordered in three categories: Prime and cue could either have similar shapes and thus also code the same side (similar-congruent condition, four combinations), different shapes but still code the same side (dissimilar-congruent condition, four combinations), or different shapes and code different sides (incongruent condition, eight combinations). All possible combinations of primes and cues are shown in Figure 2.4B, along with the side that was signaled by the cue. The prime was presented for $24 \mathrm{~ms}$ and prime-cue SOA varied within blocks in 6 steps of one frame duration from 24 to $82 \mathrm{~ms}$. 


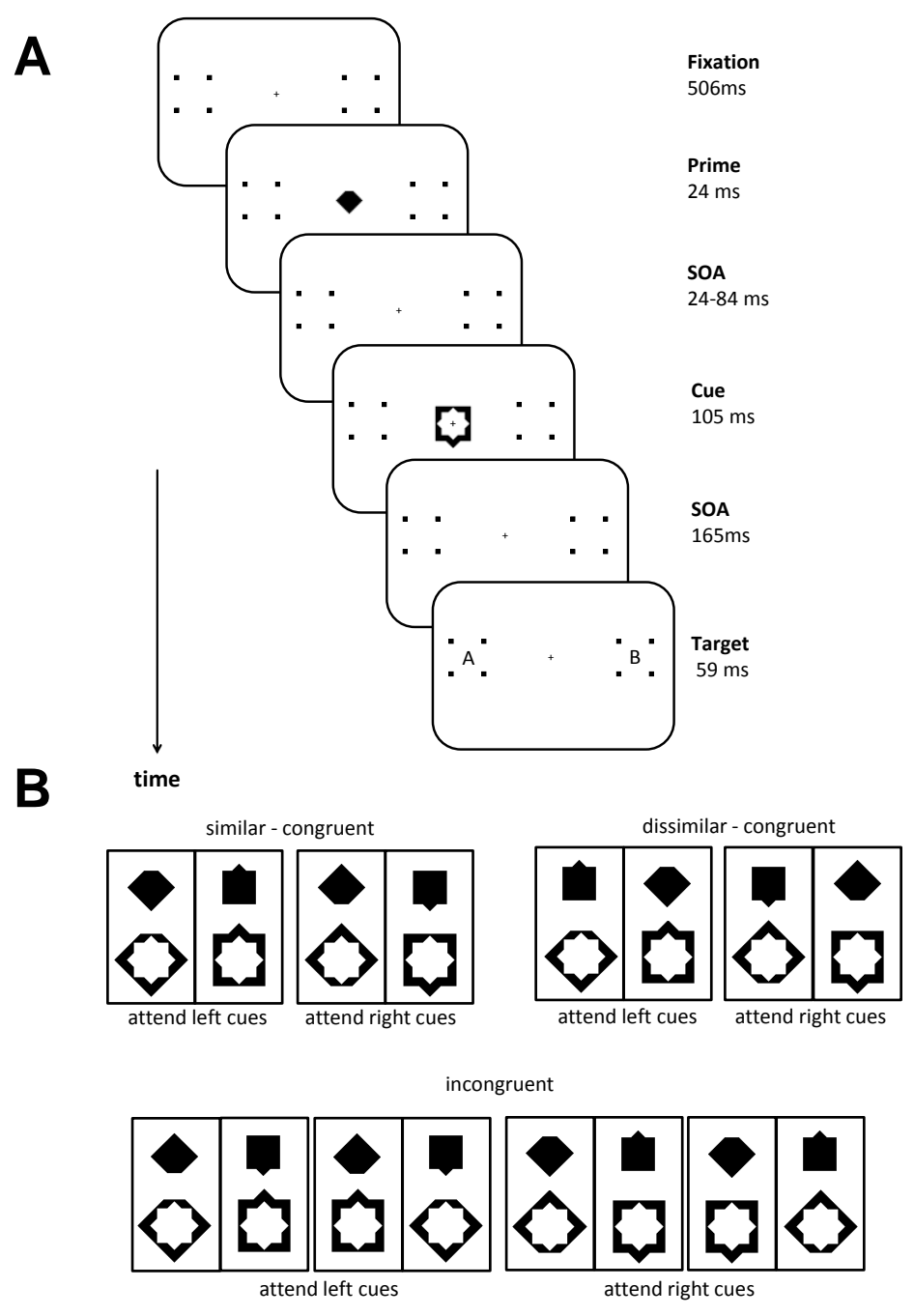

Figure 2.4 Stimuli in Experiment 3. (A) Sequence of events in a trial. (B) Possible combinations of prime and cue stimuli in the three conditions.

Design and procedure. Participants completed a total of five sessions each of which lasted between 45 and 60 minutes. The first session was considered practice and the data of this session was not analyzed. In this session we used longer cue-target SOAs to make the task easier and employed the shifting random dot background to give participants online feedback about eye movements. In the following three sessions each participant completed 16 trials with each combination of three levels of Prime-Cue Congruency (similar-congruent, dissimilar-congruent, incongruent), 6 levels of SOA, and 2 target arrays. In addition, 48 
randomly intermixed no-go trials were run, resulting in a total of 624 trials per session. Note that for every prime the similar-congruent and the dissimilar-congruent cues were twice as likely to follow as the incongruent cues. In the fifth session, participants completed the prime recognition task which consisted of the same 624 trials as in the sessions before.

Analyses. As in the previous experiments, the data were analyzed using repeated-measures ANOVA with factors SOA and Prime-Cue Congruency. Three separate ANOVAs were performed for RTs, arc-sine transformed response error rates, and arc-sine transformed rates of eye-movement errors. In order to differentiate between perceptual and post-perceptual components we computed two different priming effects. We defined the perceptual component of the cue-priming effect as the difference between dissimilar-congruent and similar-congruent trials where prime and cue indicate to attend to the same side but they are perceptually either similar or dissimilar. When comparing dissimilar-congruent to incongruent trials, however, primes are perceptually dissimilar to the cue in both cases but they indicate to attend either to the same or to different sides as the cue. Therefore, any performance difference between incongruent and dissimilar-congruent trials can be conceived as a reflection of the post-perceptual component of cue-priming effects. In order to evaluate the different cue-priming effects, we performed two additional ANOVAs on RT data comparing dissimilar-congruent trials either to incongruent or to similar-congruent trials. Finally, we conducted an ANOVA to examine whether the partial perceptual similarity in terms of global shape between prime and cue affects cue processing. To this end we compared incongruent trials on which prime and cue had the same global shape (both made of squares or both made of diamonds) to incongruent trials on which prime and cue differed in their global shape (see Figure 2.4B). We performed repeated-measures ANOVAs on dependent variables RT and 


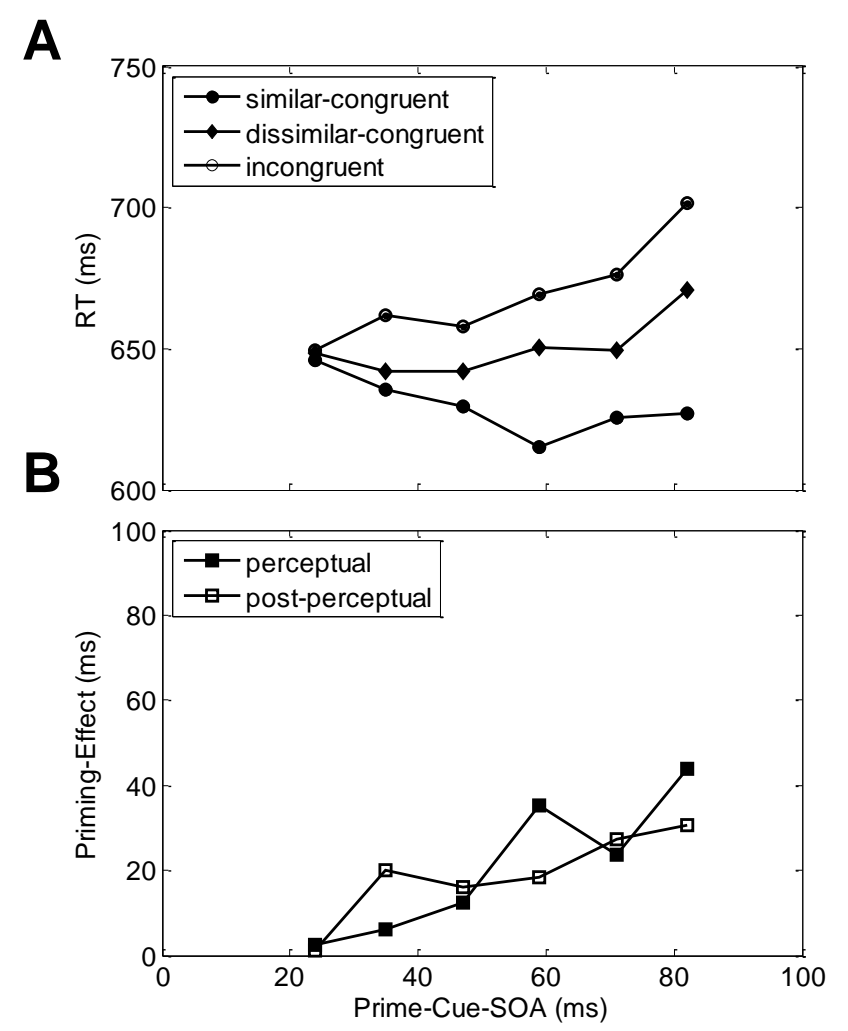

Figure 2.5 Results in Experiment 3. (A) RT as a function of prime-cue SOA and prime-cue congruency. (B) Priming effect on RT as a function of prime-cue SOA separated for the perceptual (dissimilar-congruent similar-congruent) and the post-perceptual effect (incongruent - dissimilar-congruent).

arc-sine transformed response error rate for these two types of trials with factors GlobalSimilarity (similar vs. dissimilar) and SOA.

\subsubsection{Results}

Response times and priming effects on RT in Experiment 3 are shown in Figure 2.5. A repeated-measures ANOVA of response times with the factors Prime-Cue Congruency and SOA revealed significant main effects of Prime-Cue Congruency, $F(2,30)=54.6, M S e=690$, $p<.001$, and of SOA, $F(5,55)=9.5, M S e=366, p<.001$. The interaction between PrimeCue Congruency and SOA was also significant, $F(10,150)=8.4, M S e=321, p<.001$. Both additional ANOVAs yielded significant effects of Prime-Cue Congruency: Responses on 
dissimilar-congruent trials $(650 \mathrm{~ms})$ were faster than on incongruent trials $(669 \mathrm{~ms}), F(1,15)$ $=20.8, M S e=826, p<.001$, indicating a post-perceptual component, but slower than on similar-congruent trials $(630 \mathrm{~ms}), F(1,15)=35.5, M S e=579, p<.001$, indicating a perceptual component. Thus, findings suggest that RT was affected by both perceptual and post-perceptual effects of Prime-Cue Congruency. The Prime-Cue Congruency x SOA interaction, that reflects an increase of priming effects with SOA, reached significance for the perceptual effect, $F(5,75)=7.8, M S e=282, p<.001$, as well as for the post-perceptual effect, $F(5,75)=2.6, M S e=319, p=.030$. Difference in RT between dissimilar-congruent and similar-congruent trials increased from $2 \mathrm{~ms}$ to $44 \mathrm{~ms}$ and the difference between incongruent and dissimilar-congruent trials increased from $1 \mathrm{~ms}$ to $31 \mathrm{~ms}$.

There was no main effect of Global-Similarity on $\mathrm{RT}(F<0.2, p>.7)$ indicating that the priming effect did not differ on incongruent trials with similar or dissimilar prime-cue pairings. However, we did find a complex Global-Similarity x SOA interaction, $F(5,75)=$ 4.2, $M S e=517, p=.007)$. With short and long SOAs, the RT difference between incongruent-dissimilar and incongruent-similar trials was not significant $(1 \mathrm{~ms}, 9 \mathrm{~ms}, 22 \mathrm{~ms}$, $18 \mathrm{~ms}$, and $18 \mathrm{~ms}, \mathrm{p}>.05$ in all cases, for 12, 24, 47, 71 and $82 \mathrm{~ms} \mathrm{SOA}$, respectively). With $35 \mathrm{~ms} \mathrm{SOA}$, responses were $13 \mathrm{~ms}$ faster on dissimilar than on similar trials, $t(15)=2.2, \mathrm{p}=$ .046. Thus, partial perceptual similarity between prime and cue does not seem to facilitate cue processing by the preceding prime.

Response error rates were not significantly modulated by any experimental factor $(p>$ .15 in all cases). Therefore we did not conduct additional ANOVAs to distinguish perceptual and the post-perceptual contributions to effects. Global-Similarity did not have a significant main effect on rate of response errors $(F<0.3, p>.5)$. The interaction of Global-Similarity and SOA was significant, $F(5,75)=3.2, M S e=0.027, p=.023$. With $35 \mathrm{~ms}$ SOA, there were 
more response errors on incongruent-similar than on incongruent-dissimilar trials (difference $=-1.4 \%, t(15)=2.6, p=.019)$, whereas the opposite was true with $82 \mathrm{~ms}$ SOA $(1.6 \%, t(15)=$ $-2.6, p=.019)$. None of the corresponding effects with the other SOAs was significant ($0.6 \%,-0.2 \%, 0.7 \%$ and $0.9 \%$ for $12,24,47$ and $71 \mathrm{~ms}$ SOA, respectively, $p>.1$ in all cases).

Eye-movement errors increased with SOA from $8.3 \%$ to $12.2 \%, F(5,80)=6.6, \mathrm{MSe}=$ $0.014, p<.001$. However, neither the main effect of Prime-Cue Congruency, nor any interaction of the two factors reached significance $(F<1, p>.75$ in both cases). See Table 2.3 for error rates in Experiment 3. Error rates for no-go trials were less than $3.5 \%$ in every individual participant with a mean across participants of $1.1 \%$.

Prime recognition. Eight of the 16 participants reported no awareness of the primes after the final choice RT session. All other participants reported that they had seen primes on at least some trials. A repeated-measures ANOVA of d'-values revealed that performance increased significantly (from d' $=0.45$ to d' $=0.69$ ) with increasing SOA, $F(5,75)=2.3, M S e=0.1, p$ $=.050$. Prime recognition performance was better than chance at all SOAs as revealed by $\mathrm{t}-$ tests of d' against 0 (mean d' $>0.34, t(15)>3.5, p<.002$, in all cases).

To test whether prime recognition performance was related to priming effects we assigned participants to two groups according to their mean prime recognition performance (median split; see Table 2.4). Prime recognition performance and priming effects for the good and poor prime recognizers are shown in Figure 2.6. On average, the 8 participants with poor prime recognition performance did not perform better than chance except with SOAs of $24 \mathrm{~ms}$ $\left(\mathrm{d}^{\prime}=0.36, t(7)=3.0, p=.020\right)$ and $72 \mathrm{~ms}\left(\mathrm{~d}^{\prime}=0.22, t(7)=2.5, p=.043 ; \mathrm{d}^{\prime}<0.21, t(7)<\right.$ 1.6, $p>.15$ in all other cases). In a mixed model ANOVA of RT data with prime recognition groups (good vs. poor) added as a between-participants factor, prime recognition group did 
A

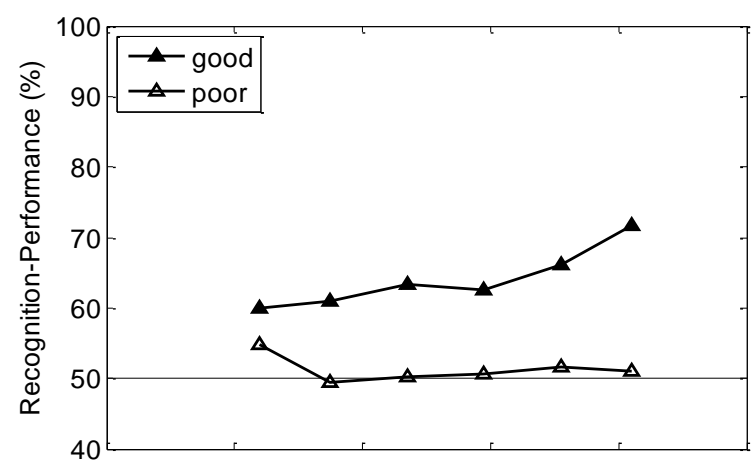

B
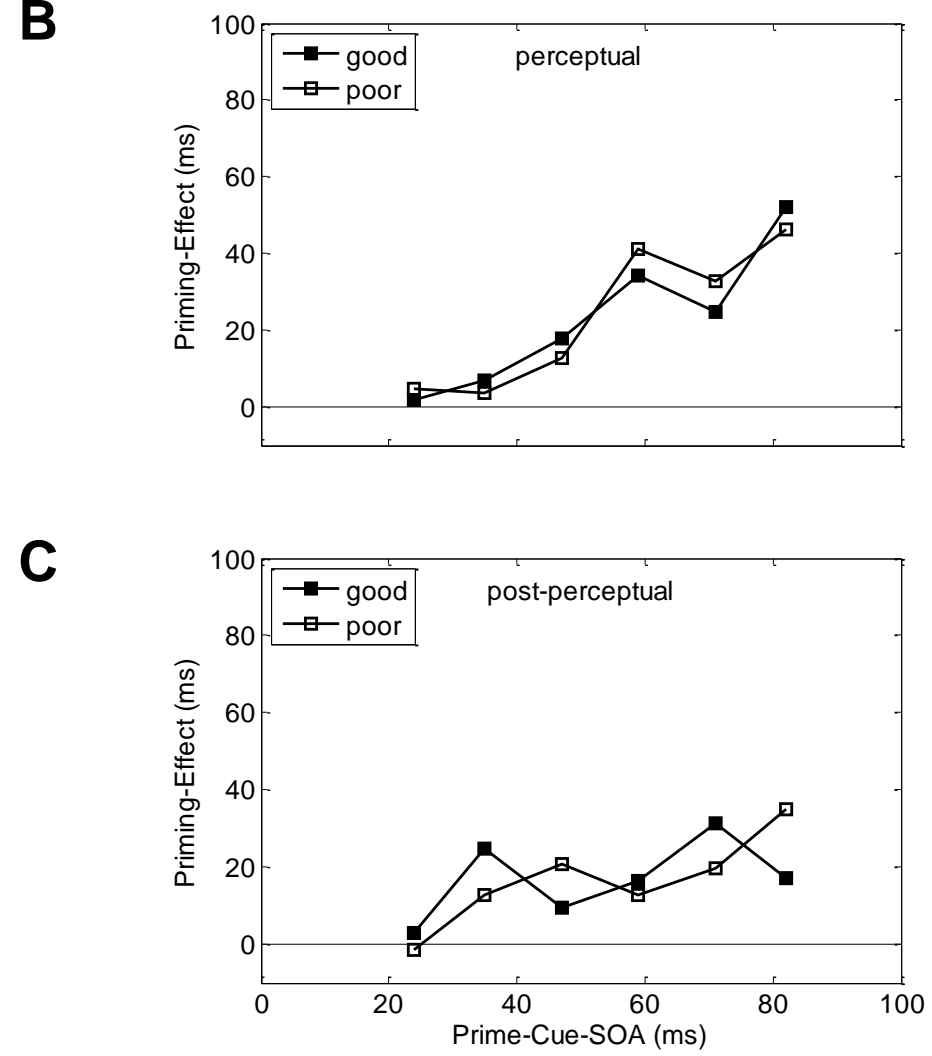

Figure 2.6 Prime recognition performance and priming effects on RT for two groups in Experiment 3 with good and poor prime recognition performance (median-split). (A) Prime recognition performance. (B) Perceptual priming effect (dissimilar-congruent - similar-congruent). (C) Post-Perceptual priming effect (incongruent dissimilar-congruent).

not have a significant main effect on RT nor did it enter into any significant interactions $(F<$ $1, p>.5$ in all cases). 
Table 2.3 Error rates and excluded trials in Experiment 3

\begin{tabular}{|c|c|c|c|c|c|c|c|c|c|c|c|c|}
\hline \multirow{3}{*}{$\begin{array}{l}\text { Prime-Cue } \\
\text { Congruency }\end{array}$} & \multicolumn{12}{|c|}{ Prime-Cue SOA(ms) } \\
\hline & 24 & 36 & 48 & 60 & 72 & 84 & 24 & 36 & 48 & 60 & 72 & 84 \\
\hline & \multicolumn{6}{|c|}{ Error Rates (\%) } & \multicolumn{6}{|c|}{ Trials Excluded (\%) } \\
\hline Similar & 1.1 & 1.7 & 1.7 & 2.6 & 2.3 & 1.7 & 8.9 & 10.1 & 9.0 & 10.3 & 10.3 & 12.3 \\
\hline Congruent & 1.5 & 1.6 & 1.8 & 1.5 & 1.4 & 2.0 & 8.1 & 9.2 & 8.8 & 9.1 & 10.7 & 12.0 \\
\hline Incongruent & 2.0 & 1.9 & 1.9 & 1.6 & 2.2 & 2.3 & 8.0 & 10.0 & 9.9 & 10.0 & 9.7 & 12.2 \\
\hline
\end{tabular}

In order to assess priming effects for the poor recognition group, we performed two additional ANOVAs on RT data from these participants comparing dissimilar-congruent trials either to incongruent or to similar-congruent trials. With these participants, a similar pattern emerged as for the whole group: responses on dissimilar-congruent trials $(659 \mathrm{~ms})$ were slower than responses on similar-congruent trials $(641 \mathrm{~ms}), F(1,7)=12.5, M S e=658, p=$ .010 , but faster than responses on incongruent trials $(680 \mathrm{~ms}), F(1,7)=20.2, M S e=511, p=$ .003. There were main effects of SOA in both analyses $(F(5,35)=3.4, M S e=240, p=.036$; $F(5,35)=5.3, M S e=578, p=.023$, for the perceptual effect and the post-perceptual effect, respectively. However, the SOA x Congruency interaction did not reach significance in these analyses, $F(5,35)=2.2, M S e=427, p=.134 ; F(5,35)=2.4, M S e=344, p=.107$, for the perceptual effect and the post-perceptual effect, respectively).

Moreover, prime recognition performance did not correlate with either priming effect on RT: $\mathrm{r}=.03, p=.913$ for the post-perceptual priming effect (incongruent minus dissimilarcongruent), and $\mathrm{r}=-.12, p=.656$ for the perceptual priming effect (dissimilar-congruent minus similar-congruent). 
Table 2.4 Prime recognition performance in Experiment 3

\begin{tabular}{ccccccc}
\hline & \multicolumn{5}{c}{ Prime-Cue SOA(ms) } \\
\cline { 2 - 6 } Group & 24 & 36 & 48 & 60 & 72 & 84 \\
\cline { 2 - 7 } & 0.45 & 0.34 & 0.46 & 0.43 & 0.57 & 0.69 \\
All participants & 0.53 & 0.59 & 0.72 & 0.73 & 0.92 & 1.19 \\
Good performers & 0.36 & 0.10 & 0.20 & 0.13 & 0.22 & 0.19 \\
\hline Poor performers & & & & & & \\
\hline
\end{tabular}

\subsubsection{Discussion}

We replicated findings from Experiment 2 and extended them by separating priming effects that are due to perceptual facilitation and priming effects that most likely arise from post-perceptual levels of processing. Although responses were fastest when primes were perceptually similar to cues, we did find a significant difference between perceptually dissimilar congruent stimuli that were associated with attending to the same side as indicated by the cue and those that were associated with the opposite side. However, the cue-priming effect was not different on incongruent trials with or without partial perceptual similarity between primes and cues. These results indicate that cue-priming of spatial attention, like cuepriming in other tasks (Mattler, 2006), cannot entirely be reduced to early perceptual levels of processing.

Experiment 3 is based on the rationale that RT differences between similar-congruent and dissimilar-congruent trials can be reduced to differences in perceptual processing of the cue in these trials. In addition, it is assumed that any perceptual difference between dissimilarcongruent and incongruent prime-cue pairs does not contribute to the RT differences between 
these trials. At first glance, there are two potential sources of perceptual priming: the global shape of the stimuli (square vs. diamond) and location of distortion (upper part vs. lower part). There was no difference in RT between global-similar and global-dissimilar incongruent trials which suggests that there was no priming effect of global similarity. According to a current theory of object recognition (Hummel, 2001) the binding of stimulus features into a structural representation of an object is time consuming and requires visual attention. However, when the visual system fails to segment an image into its parts due to inattention or insufficient processing time, a statically bound representation is generated which is invariant with translation and scale and allows rapid, automatic recognition of familiar objects in familiar views (Thoma, Davidoff \& Hummel, 2007). In this perspective, our findings suggest that perceptual recognition of the cue is facilitated by the preceding prime only if the prime is a scaled replicate of the cue. Thus it seems unlikely that similarity in the location of the distortion between globally dissimilar stimuli led to perceptual priming and contributed to differences in RT between dissimilar-congruent and incongruent prime-cue pairs. Therefore, RT differences between these types of trials most likely result from postperceptual levels of processing that need to be determined more specifically by later research.

We found that both components of the cue-priming effect increase with increasing prime-cue SOA. Prime recognition performance also increased with prime-cue SOA. Thus, we were not able to find a dissociation between priming effects and prime recognition, as has been found for motor priming (Vorberg et al., 2003) as well as cue-priming (Mattler, 2003b). Note that in principle participants' prime recognition performance could have benefited from a strategy that involves simply responding to the cue/mask stimulus, because primes were mapped to the same response as the mask on two thirds of the trials. However, no participant reported having used this strategy. Nonetheless, any response bias caused by the mask would 
lead to a measure of prime visibility which overestimates participants' true capability to discriminate the primes. Nevertheless, like Mattler (2003b, 2006) we found that priming effects were not different in participants who were superior in the prime recognition task and those participants who performed close to chance levels. Therefore, these findings suggest that the present cue-priming effects are independent from conscious perception of the primes.

\subsubsection{General discussion}

This study extends findings of Mattler (2003b, 2005) and Lau \& Passingham (2007) which showed that unconscious stimulus features can affect cognitive control processes by using a spatial cue-priming task. The present findings go beyond previous studies that reported effects of masked peripheral cues on spatial attention (McCormick, 1997; Mulckhuyse et al., 2007; Scharlau \& Ansorge, 2003) as our primes were centrally presented stimuli. Moreover, we extend evidence that central masked stimuli like arrows and eye gaze can affect orienting of attention (Cole \& Kuhn, 2010; Sato et al., 2007) by showing priming effects with arbitrary, symmetrical masked stimuli which our participants have not encountered in a spatial cueing task before. Thus, the present cue-priming effects cannot be explained by any special mechanisms that govern the processing of highly overlearned stimuli. As far as we know, this is the first study to show that shifts of spatial attention can be modulated by masked symmetrical stimuli that are centrally presented. Findings challenge the view that consciousness is needed to yield an effect of this kind of stimuli on shifts of spatial attention. We assume that peripheral and spatially compatible stimuli affect attention by mechanisms related to exogenous control of spatial attention whereas arbitrarily mapped symmetrical stimuli that are centrally presented operate due to endogenous control mechanism. Given this distinction, our findings suggest that mechanisms of endogenous control of spatial orientation are susceptible to unconscious stimulus features. 


\subsubsection{Comparison of spatial cue-priming to the traditional spatial cueing paradigm}

Is the cue-priming procedure used in the present study tapping the same or different attentional processes to those indexed by traditional spatial cueing paradigms? Important differences between the paradigms result from different validities of the visible cues. Cues are valid on $100 \%$ of the trials in our paradigm but cues are partially invalid in the traditional paradigm. In consequence, traditional studies typically used only a single target (McCormick, 1997; Posner, 1980) or distractor stimuli on non-target locations (e.g., Jonides, 1981; Mattler, 2003a, 2004; Müller \& Rabbitt, 1989). Our paradigm with 100\% valid cues allows the usage of target displays that comprise two lateral stimuli, so that participants are required to respond to the stimulus on the cued side and to ignore the accompanying stimulus on the non-cued side.

These procedural differences cause differences at processing levels. In the traditional paradigm, participants have to deal with the possibility of infrequent invalid trials. This might motivate some participants to engage only little attention to the cued side if they experience that little engagement is better than full engagement on invalid trials. After all, the target rather than the cue determines the spatial location where participants have to allocate their attention. Therefore, it might appear to some participants that it is better to ignore the cue and be alert and monitor all potential target locations because the unequivocal target display does provide sufficient information to select the target and do the task successfully without using the cue. In the traditional paradigm, faster RTs on validly cued trials than on invalidly cued trials have been taken as evidence for the view that participants allocated at least some attention to the cued side at least on part of the trials. Note, however, that such an RT difference might as well result from the irritation caused by the infrequent sequence of an invalid cue and a target stimulus that appears at an unexpected stimulus location. In line with 
this, it has been clear from the early days of the traditional paradigm (e.g., Posner, Walker, Friedrich \& Rafal, 1984) that the RT difference between validly and invalidly cued trials entails various processes in addition to the shift of attention, including recognition of the fact that the wrong spatial location is attended, disengagement from the wrong location, shifting of attention, and the new engagement at the correct spatial location. Therefore, traditional measures of endogenous shifts of spatial attention have not been conceived as a pure measure of any specific process. As mentioned in the introduction, we follow the traditional conceptual distinction and conceive shifts of attention as endogenously triggered if centrally presented symmetrical cue stimuli were used - as for instance in our experiments.

In our cue-priming paradigm, endogenous shifts of attention are measured by RTs on trials with incongruent primes minus RTs on trials with congruent primes. Compared to the traditional measure of spatial cueing, this measure reflects the operation of a different set of attentional processes. In the cue-priming paradigm, the visible cues are valid on every trial and participants need to process the cues to select the target stimulus in the two-stimulus target display by attending to the location that is reliably coded by the cue. On the one hand, stimulus processing of the cue can be modulated by the preceding prime (see Experiment 3 ). Beyond this, however, the prime might also access the same set of processes that are accessible for the cue. In this case, on congruent trials, the cue calls for the same processes as the prime and both facilitate processing of stimuli at the cued location. On incongruent trials, prime and cue direct attention to opposite locations. Mathematical analyses of similar priming effects in the response priming paradigm suggest that the interaction of the effects of prime and the following stimulus depends on the SOA between these two stimuli (Vorberg et al., 2003; Mattler \& Palmer, 2012). Thus, if there is sufficient time on an incongruent trial until the cue appears the prime might direct attention to the incorrect location. With less time 
between prime and cue, the processing of the cue might lead to the initiation of processes which might interfere at various levels with the processes that have been triggered by the prime.

In addition, model analyses suggest that the priming effect is further determined by the effect of the following stimulus. The priming effect is large if the effect of the following stimulus is comparably small, but the priming effect is small when the effect of the following stimulus is larger than the effect of the prime (Mattler \& Palmer, 2012). Such an interaction of the effect of prime and cue stimuli might account for the time course of the priming effect in the present study. On first glance, it might seem puzzling that the priming effect in Experiment 1 decreases with increasing cue-target SOA while cue validity effects in traditional spatial cueing experiments with imperfectly predictive cues typically increase with cue-target SOA (e.g., Lambert \& Duddy, 2002; Müller \& Rabbitt, 1989). In order to comprehend this difference, it is important to keep in mind that the primes in our experiments were always followed by a valid cue stimulus. If preparatory processes depend more on the cue than on the prime with longer cue-target SOAs, the decreasing cue-priming effect could result because the effect of the prime decreases with increasing cue-target SOA whereas the effect of the cue increases with SOA. Note that the effective SOA for the cue-priming effect in our experiments consists of the $170 \mathrm{~ms}$ prime-target SOA. Previous studies have reported significant cueing effects of central symmetrical cues with comparable short cue-target SOAs (e.g., Brignani, Guzzon, Marzi \& Miniussi, 2009; Lambert \& Duddy, 2002). In sum, we think that our cue-priming effect is a valid measure of processes that are involved in endogenous shifts of attention. Nonetheless, one has to mind the differences between the paradigms and the corresponding differences in the potential sets of processes that contribute to the effects when cue-priming and traditional cueing effects are compared. 


\subsubsection{Comparison of spatial cue-priming to cue-priming effects in other tasks}

Taken together, our results show that masked primes have similar characteristics in a cue-priming of spatial attention as in other cue-priming tasks (Lau \& Passingham, 2007; Mattler, 2003b, 2005; 2006). Effects are modulated by (a) cue-target SOA and (b) prime-cue SOA in a similar way, (c) do not depend on prime visibility, and (d) cannot be entirely reduced to priming of early perceptual processes. These commonalities hint at common mechanisms, possibly because all tasks require the allocation of cognitive resources, either between task sets, stimulus modalities, or stimulus locations. These commonalities challenge the view that spatial attention is "special" (e.g., Hillyard \& Anllo-Vento, 1998; Treisman \& Gelade, 1980). Instead, our findings are consistent with recent results of Chiu \& Yantis (2009; see also Esterman, Chiu, Tamber-Rosenau \& Yantis, 2009). Chiu \& Yantis (2009) compared neural activity between shifts of spatial attention and switches of categorization rules and found a common locus in medial superior parietal lobule. This was taken as evidence that a single mechanism accomplishes switches in both domains. Therefore, the similarities of spatial cue-priming effects in this study and cue-priming effects in other studies could be taken as evidence for the view that the control of voluntary attention is based on comparable processes as task switching.

The cue-priming paradigm has been criticized by the view that priming effects could possibly be explained by low level prime-cue interactions (Dehaene, 2008; Reuss, Kiesel et al., 2011). However, there is behavioral (Mattler, 2006; Reuss, Kiesel et al., 2011) as well as neurophysiological evidence (Lau \& Passingham, 2007) suggesting that cue-priming of task sets extends beyond low level processes. Our Experiment 3 provides similar evidence for priming of spatial attention. In this experiment, masked primes still affected shifts of spatial 
attention when we controlled for low level prime-cue interactions by manipulating prime-cue similarity.

\subsubsection{Do primes affect voluntary attention?}

As outlined in the introduction, recent evidence indicates that centrally presented stimuli can affect shifts of attention reflexively even when they are non-predictive for target location if arrows or eye gaze stimuli are used (Friesen \& Kingstone, 1998; Tipples, 2002) which might result from exogenous mechanisms of attentional control (e.g., Kingstone et al., 2003; Reuss, Pohl et al., 2011). These kinds of involuntary, reflexive automatic cueing effects have been distinguished from voluntary shifts that are governed by endogenous control mechanisms because effects require an interpretation of the meaning of the stimuli. Primes in our experiments were also non-predictive for target location, whereas cues in our experiments were always valid and thus likely triggered voluntary shifts of attention. Therefore, the question arises whether our primes modulate attention via mechanisms of voluntary or involuntary attention.

At first glance, the present priming effects might be considered an instance of involuntary effects, because primes were not predictive for target locations in any way and they were outside of participants' awareness. Moreover, priming effects are largest with short cue-target SOA which is the opposite of what is typically found in endogenous cueing. This could be taken as evidence that priming effects are based on involuntary mechanisms but it might also result from other differences between traditional experiments studying effects of cue validity with single targets and our cue-priming experiments in which cues are needed to select among two competing letters. In this paradigm, however, the only reason for participants to associate a square prime with a shift of attention to the left is that they are prepared for a square cue to signal that the target will be on the left side. The fact that priming 
effects depend on participants' intentions to shift their attention according to cues makes us believe that the reported cue-priming effects result from endogenous mechanisms. The Direct Parameter Specification hypothesis of Neumann (1990) suggests that unconscious primes can be effective by virtue of the same processes as visible stimuli. Even though Direct Parameter Specification was devised to explain priming effects on motor responses, Scharlau and Ansorge (2003) employed Direct Parameter Specification to explain effects of masked stimuli on exogenous attention in a similar way. These authors used square and diamond shaped stimuli and presented them peripherally, at the same location as the targets. Priming effects were interpreted as evidence for the view that unconscious primes can capture attention through Direct Parameter Specification. Mattler (2003b) employed Direct Parameter Specification to explain cue-priming of cognitive control operations. This view is supported by the finding that cue-priming effects share several characteristics with response priming effects (Mattler, 2003b). To the extent that primes are effective by virtue of the same processes as cues, it seems likely that primes affect voluntary attention. This view is consistent with recent neurophysiological evidence which suggests that unconscious stimuli can affect cognitive control operations (e.g., Krüger et al., 2012; Lau \& Passingham, 2007; Van Gaal et al. 2008; 2009).

\subsubsection{Locus of cue-priming effects on shifts of spatial attention}

Mattler (2003b) distinguished three possible loci for cue-priming effects. Applying this view to the experiments at hand, primes could either act on perceptual levels by modulating cue identification, either on central levels by activating representations of left or right, or on post-central levels by initiating shifts of spatial attention. Experiment 3 showed that congruent primes have larger effects when they are replicas of the cue. This indicates that part of the priming effects does indeed result from a perceptual level of processing. However, 
primes have effects beyond perceptual similarity as indicated by the difference of the effect between incongruent and congruent primes in Experiment 3 that were both perceptually dissimilar. These findings indicate that the effects of primes go beyond simple perceptual levels of processing. However, the present findings do not suffice to specify the exact level of processing that generate the later priming effect. The effect might arise from levels of processing which are considered by some as high level perceptual processing, by others as categorical levels of processing. For instance, it has been argued that cue-priming effects with perceptually dissimilar stimuli like those of Mattler (2006) result because stimuli that are associated with the same task prime each other at a level where they share the same category (Reuss, Kiesel et al. 2011). Applied to the present experiment, it would mean that dissimilar primes are effective not because they affect the allocation of attention, but because they affect cue processing at a categorical level. In sum, the present priming effects could result from congruency effects at categorical levels of processing, at decision levels, at control levels, or at later levels by actually initiating shifts of attention. Further research is needed to clarify this issue.

\subsubsection{The role of prime visibility.}

Masking was not particularly effective in our experiments and overall there was above chance performance in the recognition tasks. We used a discrimination task in the prime recognition sessions of our experiments to capture information relevant to the choice-RT task that produced the cue-priming effect (Schmidt \& Vorberg, 2006). A previous comparison between prime detection performance and prime recognition performance in metacontrast masking revealed that participants performed even better in the prime detection task (Vorberg et al., 2003). In Experiment 3, half of the participants reported after the priming sessions that they were unaware of the presence of the primes during the priming sessions. Only during the 
following prime recognition session participants demonstrated that they were able to discriminate primes when they were instructed to attend to primes' crucial features. This behavioral difference indicates that the present approach is rather conservative: if any participant cannot even discriminate primes in a condition of this type of recognition task, it is good evidence for the view that this participant has not been aware of the crucial features of the effective stimuli in the choice-RT session. On the other hand, however, if someone performs well in the prime recognition session, it cannot be concluded that this participant was aware of the prime during the choice-RT session. According to the present approach, however, it would be sufficient if participants cannot discriminate the stimulus features that produce the priming effect to infer that these unconscious stimulus features generated the priming effect in the choice-RT task (Schmidt \& Vorberg, 2006). Note that it is rather difficult to yield chance level prime recognition performance with this type of metacontrast masking task, when performance is measured carefully with profound initial instruction, trial by trial feedback, and a long series of more than 600 trials (e.g., Mattler, 2003b). It is more likely to find individual differences in metacontrast masking (Albrecht, Klapötke \& Mattler, 2010).

There is some evidence in our data indicating that in some participants of Experiment 3 with $36,48,60$, and $84 \mathrm{~ms}$ SOA the crucial features of the primes were not discriminable and these participants nonetheless produced significant cue-priming effects in the choice-RT task with these stimulus conditions. Moreover, it is important to mind that the fact that primes were discriminable for other participants does not mean that prime visibility is crucial for cuepriming effects. Several response priming studies demonstrated that priming effects and prime recognition performance are independent processes (e.g., Albrecht et al., 2010; Vorberg et al., 2003; Mattler, 2003b). In addition, cue-priming effects in other tasks have been found to be 
independent of prime visibility too (Mattler, 2003b) and can increase with SOA even when prime visibility decreases with SOA. Most important, cue-priming effects did not differ between participants with good and poor prime recognition performance in Experiment 3 (Figure 2.6). This is consistent with the view that unconscious stimulus features can modulate endogenous shifts of attention.

\subsubsection{Conclusion}

We showed effects of central symmetrical masked primes on shifts of spatial attention that do not depend on prime visibility. These effects are similar to cue-priming effects in other tasks (Mattler, 2003b, 2005) suggesting a similar mechanism for priming of spatial as well as non-spatial attention. Furthermore, these cue-priming effects extend beyond low level priming based on prime-cue relationship. Whereas physiological studies already provided evidence for post-central effects of masked primes in cue-priming, it remains open for future research to determine the locus of effects in the case of spatial cue-priming. The cue-priming paradigm provides a new tool to investigate the effects of masked cues on spatial attention.

\subsection{Summary of Project 1}

In a first attempt to transfer cue-priming to a spatial task we investigated effects of cue-target SOA, prime-cue SOA and perceptual similarity in three separate experiments using a letter discrimination task with distractors. This task is similar to other cue-priming experiments (Mattler, 2003; 2005) and magnitude and time course of the present spatial cue-priming effects were also very similar. They decreased with increasing cue-target SOA increased with prime-cue SOA and were in part, but not entirely based on perceptual similarity of prime and cue symbols. 
In all three Experiments target displays consisted of letters A and B being presented, one to the left and one to the right of fixation. Before every target display a cue symbol indicated which side was relevant and participants were instructed to use the cues to focus their attention on the corresponding side. For instance, in Experiment 2 a square always cued left and a diamond right. Participants then had to indicate whether the letter presented on the cued side had been A or B. Crucially, before every cue another symbol was presented very briefly - the prime. Primes were the same symbols as cues, e.g., in Experiment 2 primes were also squares and diamonds. These primes were smaller than the cues and were masked by the cues. This was made possible because the cues had cut-outs that fit the primes. Primes were thus difficult to see due to a phenomenon called metacontrast masking. Trials on which prime and cue are the same symbol are called congruent trials and trials on which they are different are called incongruent trials. The effect of primes can be seen in the difference between congruent and incongruent trials. Given the symbolic nature of the primes and cues, it was assumed that they would affect endogenous attention.

We found that spatial cue-priming effects with square and diamond stimuli exhibit similar characteristics as cue-priming effects in other tasks. In Experiment 1, priming effects decreased with increasing cue-target SOA whereas the increased with increasing prime-cue SOA in Experiment 2 This pattern of results can be explained by assuming that the contribution of primes relative to the contribution of cues to the allocation of attention is dependent on the relative amount of time they are given before the next stimulus. Increasing cue-target SOA increases only the relative contribution of the cue. In contrast, variation of prime-cue SOA increases relative contribution of the prime. However, the effect of cue-target SOA seems to be at odds with what is known from spatial cueing studies. There, effects of cue validity on spatial attention typically increase with cue-target SOA. 
In Experiment 3 we varied perceptual similarity of primes and cues. To this end we used four different symbols with two being associated to each side.

Results show that masked primes can affect endogenous spatial attention. Further, similarities between the present and previous cue-priming experiments suggest similar underlying mechanism which suggests similarity between spatial attention and other types of attention. Problems in the interpretation of results from Project 1 arise from the fact that the task we used here is not a typical spatial attention task. It can be argued that in this task with relevant distractors cues are mainly used to select the correct target letter and not so much to direct spatial attention in advance of target presentation. This might explain the surprisingly large priming effects with short-cue-target SOAs which are at odds with findings from other spatial cueing experiments where effects of cue validity increase with cue-target SOA. Thus, Project 2 was designed to assess spatial cue-priming effects in a task that is more typical for the study of spatial attention. 


\section{Project 2 - Spatial cue-priming effects on accuracy in signal detection tasks}

The following section 3.1 appeared as 'On the source and scope of priming effects of masked stimuli on endogenous shifts of spatial attention' in 'Consciousness and Cognition' (Palmer \& Mattler, 2013b.

\subsection{Experiments 4 and 5 - On the source and scope of priming effects of masked stimuli on endogenous shifts of spatial attention'}

\subsubsection{Abstract}

Unconscious stimuli can influence participants' motor behavior as well as more complex mental processes. Previous cue-priming experiments demonstrated that masked cues can modulate endogenous shifts of spatial attention as measured by choice reaction time tasks. Here, we applied a signal detection task with masked luminance targets to determine the source and the scope of effects of masked stimuli. Target-detection performance was modulated by prime-cue congruency, indicating that prime-cue congruency modulates signal enhancement at early levels of target processing. These effects, however, were only found when the prime was perceptually similar to the cue indicting that primes influence early target processing in an indirect way by facilitating cue processing. Together with previous research we conclude that masked stimuli can modulate perceptual and post-central levels of processing. Findings mark a new limit of the effects of unconscious stimuli which seem to have a smaller scope than conscious stimuli. 


\subsubsection{Introduction}

Numerous priming studies have provided evidence for the view that humans can process unconscious visual stimuli to a degree that unconscious information influence motor behavior (e.g., Klotz \& Neumann, 1999; Mattler, 2003; Mattler \& Fendrich, 2007; Neumann \& Klotz, 1994; Schmidt, 2000, 2002; Vorberg, Mattler, Heinecke, Schmidt, \& Schwarzbach, 2003, 2004; Wolff, 1989) and also more complex mental operations (e.g., Mattler, 2003; 2005). In these studies a visible imperative stimulus has been preceded by a masked prime stimulus that was either associated to the same (congruent) or to opposite operations (incongruent) as the visible stimulus. A performance difference between incongruent and congruent trials was taken as evidence for an effect of the masked prime stimuli (Wolff, 1989). These findings have been taken as evidence for effects of unconscious stimuli because priming effects have been dissociated from the performance in prime recognition tasks in respect to their time-course (e.g., Mattler, 2003; Vorberg et al., 2003) and individual differences in prime recognition tasks (e.g, Albrecht \& Mattler, 2010; Mattler, 2003). In addition, several studies reported priming effects in conditions where participants' prime recognition performance was at chance levels (e.g., Vorberg et al., 2003; Mattler, 2003; Mattler \& Fendrich, 2007). However, the mechanisms underlying these priming effects are still not entirely clear. The present study contributes to this research by demarking the source and scope of the effects of unconscious stimuli on shifts of spatial attention. On the one hand, this relates to the issue of localizing where priming effects of masked stimuli arise (Mattler, 2006). On the other hand, the signal detection task offers an opportunity to determine where the effects of unconscious stimuli end. 


\subsubsection{Priming effects on executive control processes and spatial attention}

Spatial attention, like eye movements, supports our ability to select relevant information from the wealth of stimuli in the environment. Psychological research has examined the mechanisms of spatial attention in distinction to eye movements with the spatial cueing paradigm (e.g., Posner, 1980). In these experiments participants have to shift their attention covertly without moving their gaze to detect or identify visual target stimuli which appear at one of several possible spatial locations on a computer monitor. Additionally, participants have to shift their attention according to a previously given cue which informs them about the likely location of the subsequent target stimulus (e.g., on the left or right side of the monitor). On most trials, the target appears at the cued spatial location (validly cued trials) and participants' performance is better than on those few trials were the target appears at the un-cue location (invalidly cued trials). This cueing effect indicates that participants used the cues to shift their attention and benefited from a valid cue. Cueing effects have been observed with cues that appeared at a potential target location but also with centrally presented symbolic cues. However, an early distinction has been made between those processes that are involved in shifts of attention that were triggered by peripheral cues presented at target location (exogenously triggered shifts) and shifts that follow on cues presented at central location (endogenously triggered shifts; Jonides, 1981). It has been assumed that exogenously triggered shifts are based on rather automatically operating mechanisms whereas endogenous shifts require an interpretation of the cue and executive control processes which realize the instructed meaning of these stimuli (e.g., Jonides, 1981). More recent evidence suggests that spatially corresponding central cues like arrows and eye gaze can affect spatial attention rather automatically as well (Friesen \& Kingstone, 1998; Tipples, 2002). Here, we employed a paradigm with symmetrical central cues designed to 
induce endogenous shifts of attention which allows an examination of executive control processes.

Recently, several studies have provided evidence that masked stimuli can affect executive control processes (Krüger, Klapötke, Bode \& Mattler, 2013; van Gaal, de Lange \& Cohen, 2012; van Gaal \& Lamme, 2012) which were previously thought of as requiring consciousness (e.g., Jack \& Shallice 2001; Dehaene \& Naccache, 2001). For instance, Van Gaal and Lamme (2012) proposed that high-level effects of unconscious stimuli can be explained as resulting from stimuli that are processed in a fast forward sweep which reaches even pre-frontal brain areas. Voluntary or endogenous orienting of spatial attention can be considered one example for executive control processes. Effects of unconscious stimuli on spatial attention have been demonstrated in previous research (see Mulckhuyse \& Theeuwes, 2010, for a review). However, these effects were mostly found with peripheral or spatially compatible cues which are known to affect attention in an involuntary, exogenous manner. In a recent series of experiments we have shown that masked symmetrical primes that were presented in the centre of the screen can nonetheless affect shifts of spatial attention (Project 1). In two experiments, a visible cue (square or diamond in outer shape) prompted participants to shift attention to either the left or the right side of the screen. Afterwards, two letters (A and $\mathrm{B}$, one on each side of the monitor) were presented and participants had to indicate in a speeded choice reaction time task whether the letter on the cued side was A or B. Each cue was preceded by a prime stimulus which shared the outer shape of the visible cues (square or diamond shaped). Thus, the outer shape of the prime was either the same as that of the cue (congruent trials) or the two stimuli differed in their outer shape (incongruent trials). On congruent trials, participants responded faster to the target stimuli than on incongruent trials. This finding indicated that masked primes can affect endogenous shifts of spatial attention. 
Moreover, the variation of the perceptual similarity between prime and cue stimuli revealed that cue-priming effects in this task arise from both, perceptual and post-perceptual levels of processing. However, the scope of the effects of primes remained unclear, because given the priming effects on choice reaction time we cannot distinguish between priming-effects on early or late levels of spatial selection.

\subsubsection{Early and late selection by spatial attention}

Validity effects in traditional spatial cueing experiments on target detection and target discrimination performance have been explained by both early and late selection accounts. Early selection accounts propose that the early perceptual processing of unattended stimuli is halted or attenuated relative to the processing of attended stimuli at early perceptual levels (Kahneman \& Treisman, 1984; Laberge \& Brown, 1989). According to one view, stimulus processing at attended locations is more efficient because of amplification of neural signals and relatively increased signal-to-noise ratio (e.g., Hawkins et al., 1990, Hillyard, Vogel \& Luck 1998). Late selection accounts, in contrast, assume that early perceptual processing of attended and unattended stimuli does not differ. Attended input is prioritized only at later stages of processing (e.g., Duncan, 1980; Shaw, 1984). According to one view of late selection accounts, attentional selection occurs at post-perceptual levels of target processing by relative weighting of input for decision or response processes (Shiu \& Pashler, 1994).

Based on physiological measures, however, current theories assume that attentional selection can be located at various levels of processing depending on the task at hand (e.g., Desimone \& Duncan, 1995; Duncan, 2006). One specific account is the perceptual load theory of attention (Lavie, 1995; Lavie \& Tsal, 1994) which assumes that the locus of attentional selection depends on the amount of perceptual load in the given task. According to this view, attentional selection operates at early levels of processing when the perceptual load 
is high and selection operates on late levels of processing when perceptual load is low. A somewhat similar notion was put forward by Luck \& Hillyard (2000) who proposed that attention operates at those stages of stimulus processing at which interference occurs.

To determine the level of attentional selection in a given task, several behavioral approaches have been established. Behavioral evidence for the view that spatial attention leads to signal enhancement (early selection) comes from studies that combined spatial cueing with a masked signal detection task (Hawkins, Shafto \& Richardson, 1988; Hawkins et al. 1990; Luck et al., 1994). In these experiments participants were given a spatial cue indicating the probable location of a following bright target stimulus which was immediately masked afterwards. Participants had to indicate whether a target stimulus was presented or not. For instance, in one of these experiments (Luck et al., 1994, Experiment 3) arrows were presented as cues in the centre of the screen pointing at either one or all of four possible target locations. After a short variable delay a white dot appeared as a target at one of these locations which was immediately followed by a random line mask which also served as a post-cue that enabled participants to determine the relevant location in the given trial. Participants were instructed to use the cues which predicted target position correctly on $76 \%$ of the trials. Consistent effects of cue validity on target detection performance were interpreted as evidence for attentional effects on early perceptual processing. These effects are hard to explain by late selection mechanisms because prioritized processing of input from attended locations should not lead to effects on detection accuracy when participants know the relevant location due to the post-cues. Moreover, experiments by Carrasco and colleagues (e.g., Carrasco, Ling \& Read., 2004; Yeshurun \& Carrasco, 1998) showed that spatial attention increases the perceived contrast of visual stimuli consistent with the idea that attending to a spatial location modulates early perceptual processing of the signals at the attended location. 
Further support for signal enhancement is given by electrophysiological studies that used event-related potentials to investigate spatial attention (e.g., Heinze, Luck, Mangun \& Hillyard, 1990; Luck, Heinze, Mangun \& Hillyard, 1990; Luck et al., 1994; Störmer, McDonald \& Hillyard, 2009) which showed attentional modulation of early target related cortical responses in extrastriate brain areas.

\subsubsection{Early or late selection in the spatial cue-priming paradigm}

As mentioned above, previous choice reaction time experiments have provided evidence for the view that masked primes can also modulate endogenous shifts of spatial attention (Project 1). However, the scope of these spatial cue-priming effects is presently unclear. The allocation of attention which produced the observed cue-priming effects on reaction time (RT) could have resulted by priming effects on early and/or late levels of selection. Congruent (incongruent) primes might have improved (reduced) the signal to noise ratio at the cued location and thus facilitate (hinder) target processing (early selection). Alternatively, primes might have affected later decision stages, e.g., by affecting the order in which perceptual evidence has been processed from each location (Müller \& Humphreys, 1991; late selection).

In previous cue-priming experiments (e.g., Mattler, 2003; Project 1) the focus of analysis was on cue-priming effects on response speed but not on target detection accuracy. In the cue-priming task employed in Project 1, perceptual load was rather low and the main cause of interference was the irrelevant letter presented on the non-cued side. Following the perceptual load theory of attention (Lavie, 1995) the cue-priming effects in Project 1 might have resulted entirely from late selection processes, if the load was low in this task. On the other hand, however, if the perceptual load would have been sufficiently high, the cuepriming effects might result from effects on early selection. The latter view is in line with 
studies that have found evidence for early effects of spatial cues and used targets that were difficult to identify, either because of target masking (e.g., Luck et al., 1994), weak or largely similar target alternatives (e.g., Mangun \& Hillyard, 1991), or the presence of distractor stimuli (e.g., Lavie, 1995).

Cue-priming effects on shifts of spatial attention is a promising experimental paradigm to address the question how far cue-priming can go, that is to determine the scope of cuepriming effects, because evidence for top-down effects on early levels of target processing has been provided mainly in the domain of spatial attention. For instance, Hillyard \& Anllo-Vento (1998) suggested that spatial attention is special because it allows selection at early levels of perceptual processing. Therefore, we reasoned that cue-priming of spatial attention might provide a unique paradigm to examine whether unconscious stimuli can access high-level cognitive control processes which allocate attention and produce an observable effect at rather low-levels in the visual processing hierarchy. In line with the view of Van Gaal and Lamme (2012), effects of masked primes on attention at early levels of target processing might be taken as evidence that feed forward processing of masked stimuli can go beyond central levels of processing and modulate top-down effects on early levels of target processing.

This determination of the scope of the effects masked primes, however, is related to the source of priming effects. On the one hand, masked primes might directly modulate early target processing if the prime is processed like a visible cue including all the effects of cues. On the other hand, however, masked primes might modulate early target processing only indirectly by an effect on the cue stimulus that is propagated to the effect of the cue. Note that both effects could also take place (see Mattler, 2006; Project 1). To distinguish between these alternatives the sources of priming effects have to be examined. 


\subsubsection{The source of cue-priming effects}

A number of studies outlined the general preconditions for the occurrence of priming effects of masked stimuli which comprise top down processes including task set, attention, and task context (see Klapötke, Krüger \& Mattler, 2011). When these preconditions are gratified, however, the levels of processing that contribute to the priming effect remain to be specified. According to the information processing approach, perceptual, central, and postcentral levels of processing are distinguished. The literature provides evidence for priming effects on each of these processing levels (see Klapötke et al., 2011, for details). A perceptual source of at least part of the priming effect is suggested by the finding that the perceptual similarity between the prime and the following imperative stimulus increases priming effects (Bodner \& Dypvik, 2005; Mattler, 2006; Project 1; see Van den Bussche, Van den Noortgate and Reynvoet, 2009, for similar findings in the domain of semantic priming). A central source contributing to priming effects is suggested by physiological studies. Lau and Passingham (2007) recorded functional magnetic resonance imaging (fMRI) data which revealed cuepriming effects on the activity of mid-dorsolateral prefrontal cortex, which has been associated with cognitive control operations (e.g., Botvinick, Cohen, \& Carter, 2004). Electrophysiological studies of both, Dehaene et al. (1998) and Leuthold and Kopp (1998) recorded priming effects on the latency of a late positivity at central electrodes $\mathrm{Cz}$ and $\mathrm{Pz}$, respectively, which has been interpreted as evidence for priming effects on post-perceptual processes, late perceptual categorization, or response conflict. Finally, individual gray-matter density in preSMA is negatively correlated with the size of individuals' priming effect (van Gaal, Scholte, Lamme, Fahrenfort, \& Ridderinkhof, 2011). Direct post-central priming effects have been reported mainly for the motor system (e.g., Dehaene et al., 1998; Leuthold and Kopp, 1998; Eimer, 1999) but also in non-motor areas in the left ventral premotor area, left inferior frontal cortex, and middle temporal gyrus (Lau \& Passingham, 2007). Taken together, 
the literature provides evidence for the view that masked stimuli can directly modulate perceptual, central, and post-central levels of processing. In our previous choice reaction-time study (Project 1), we found that spatial cue-priming effects can in part be explained as arising from perceptual priming of cue processing but also from post-perceptual processes. Postperceptual cue-priming effects could lead to direct priming effects on early levels of target selection. Perceptual priming effects, in contrast, could modulate signal detection performance only indirectly if the prime induced modulation of cue processing propagates some effect to the allocation of spatial attention.

\subsubsection{Aim of the present study}

By combining cue-priming and a signal detection task, we attempted to gain further insight into the source and limits of cue-priming effects on shifts of spatial attention. Priming effects on early visual processing would suggest an effect of masked primes on the allocation of attentional resources, a mechanism that goes beyond a decisional (late-) selection mechanism associated with voluntary attention (Prinzmetal et al., 2005). Our experimental design resembles the design used by Luck et al. (1994) in their third experiment. In contrast to this previous experiment, which used the performance differences between validly and invalidly cued trials as a measure of shifts of attention, we used visible cues that were valid on each trial. Moreover, we presented masked primes before the visible cues which were either congruent or incongruent to the cues. In consequence, we examined the performance difference between congruent and incongruent trials as an index of priming effects on shifts of spatial attention. This cue-priming method is similar to the experiments in Project 1 . The present study, however, differs from our previous study in terms of task and dependent variables. In the previous study we used a speeded choice reaction-time task and measured RTs and response accuracy; in the present study we employed a target detection task which 
was considered to be better suited as an indicator of the scope of priming effects on early levels of signal processing. We used signal detection methods to examine d' as an index of sensitivity and $\mathrm{c}$ as a measure of decision criterion in order to investigate possible biasing effects of cue-priming. In Experiment 4 we aimed to establish whether spatial cue-priming effects in a signal detection task exhibit similar characteristics as in the choice reaction time task. In Experiment 5 we tried to distinguish perceptual and post-perceptual sources of the priming effects on signal detection performance.

In summary, the present experiments were designed to help answering the questions: How far does the visual system process masked primes? Do masked primes modulate only perceptual processing of the following cues, or do they modulate processing up to central decision levels and executive control systems where cues are processed, or even on postcentral levels of processing? Do masked primes have an effect on attentional selection in terms of late levels of target selection, or even down to early levels of target processing.

\subsubsection{Experiment 4}

Experiment 4 combined cue-priming with a spatial cueing task to investigate whether masked prime stimuli have access to executive control processes in such a way that they modulate detection performance of masked luminance targets. Results from cueing experiments with similar target detection tasks have been interpreted as evidence for signal enhancement (Hawkins et al., 1990, Luck et al., 1994). Cue-priming effects are known to increase with prime-cue SOA and decrease with cue-target SOA (Mattler, 2003; 2005; Project 1). Therefore, we manipulated both SOAs at the same time to see whether priming effects would show similar variation with SOA in this task. 


\subsubsection{Method}

Participants. 12 participants (11 women; age 19-21, M = 19.8) completed 3 sessions of the experiment in exchange for course credit or payment of $€ 42$. All had normal or corrected to normal vision. Three additional participants were excluded after the practice session because they committed more than $65 \%$ errors.

Task. (1) In the target detection task, participants had to detect masked luminance targets that were presented on $50 \%$ of all trials. On each trial, they indicated that a target was present by pressing the "+" key and that a target was absent by pressing the "Enter" key on the number pad of the keyboard. There was no time limit to respond on each trial. Before each target, participants received a cue (square or diamond) that predicted the target location with $100 \%$ validity. Before each cue a prime was briefly presented which was metacontrast masked by the following cue. The shape of the primes was either congruent or incongruent to the outer shape of the cue. Participants were asked to keep fixation on a mark in the centre of the screen and fixation was controlled using an eye tracking camera. In order to allow precise measurement of eye movements, participants were instructed to rest their head in a chinrest and to avoid movements during experimental blocks. (2) In the prime recognition task, participants had to indicate the identity of the prime stimulus (square or diamond) using the same keys as before without speed stress. They had to wait until target onset to give their response. Participants were not informed about the presence of primes until after the second target detection session.

Stimuli. Figure 3.1 illustrates an example of an experimental trial. Trials started with a fixation cross $\left(0.26^{\circ}\right)$ that was presented for $500 \mathrm{~ms}$. Then prime (24 ms) and cue stimuli (105 ms) followed in the centre of the screen. Prime-target-SOA was held constant at $400 \mathrm{~ms}$ and prime-cue-SOA was varied in 4 steps (24 ms, $35 \mathrm{~ms}, 71 \mathrm{~ms}$, and $82 \mathrm{~ms})$. In consequence, cue- 


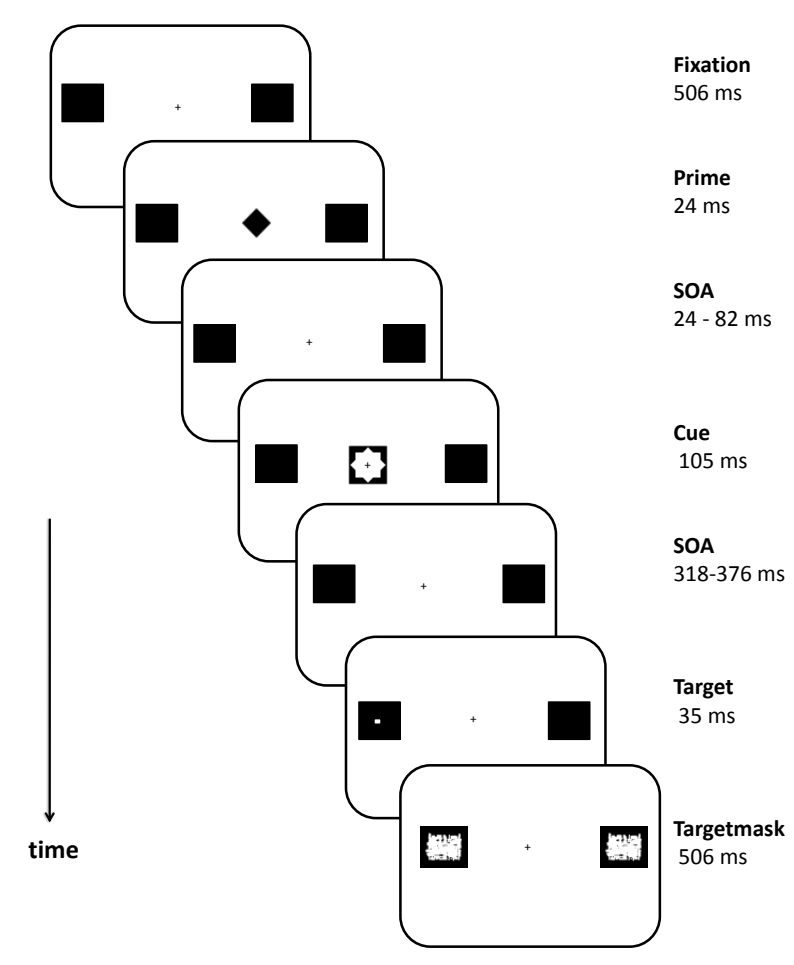

Figure 3.1 Sequence of stimulus events in a trial of Experiment 4 .

target-SOA also varied in 4 steps (376 ms, $365 \mathrm{~ms}, 329 \mathrm{~ms}$, and $318 \mathrm{~ms})$. Targets were presented with $7.1^{\circ}$ eccentricity at the cued side for $35 \mathrm{~ms}$ and were followed by masks at both sides which were presented for $500 \mathrm{~ms}$. Prime and cue stimuli were black squares and diamonds (edge to edge: $1.9^{\circ}$ for targets, $1.1^{\circ}$ for primes) presented on a light grey background. Prime stimuli fit into the cutout of cues with one line of pixels in between. This allowed cues to serve as metacontrast masks for the primes. Target stimuli were small bright squares $\left(0.2^{\circ}\right)$ presented at the centre of black squares $\left(3^{\circ} \times 2.7^{\circ}\right)$, which were masked by white random line masks $\left(2.4^{\circ} \times 2.1^{\circ}\right)$. Masks always appeared on both sides and were randomly chosen for each trial from a set of 100 different random line masks. Target luminance was adapted to participants' performance after each block so that they would answer correctly on about $75 \%$ of trials. Thus, target luminance was the same for congruent and incongruent trials. 
Design and procedure. Participants were introduced to the task in a practice session of about 1 hour. In a short block of trials at the start of the practice session two complementary black and white random-dot patterns were presented as rapidly alternating background like in our previous experiments (Project 1). This flickering background provided participants with online feedback about their eye movements because the rapidly flickering complementary dot-patterns appeared as a homogenously grey background when participants did not move their eyes but as a zigzag-pattern when participants moved their eyes (Guzman-Martinez, Leung, Franconeri, Grabowecky \& Suzuki, 2009). This was explained to participants and used to train them to do the task holding fixation without moving their eyes. Over the course of the practice session target luminance decreased which led to increasing task difficulty. During the main experimental session participants completed a total of 2560 trials in the priming session which was segmented in 4 parts of 640 trials each. After each segment, there was an obligatory rest period during which participants could leave the room. Each of the four segments comprised 10 blocks of 64 trials. Each possible combination of 2 primes, 2 cues, 2 targets and 4 SOAs was presented twice in each block, resulting in a total of 320 congruent and 320 incongruent trials with each SOA. Initial target luminance was chosen after each participants' final value in the practice session, in which luminance was constantly adapted using a three-up, one-down procedure which means that target luminance increased by three steps after each incorrect response and decreased by one step after each correct response (Kaernbach, 1991). In the experimental session, target luminance was adjusted after each block of trials: luminance was increased if participants performed better than $80 \%$ correct in the previous block and decreased if they performed worse than $70 \%$ correct. The entire third session was used to estimate participants' prime-recognition performance. In this session each participant completed 640 trials of the prime recognition task. 
Apparatus. Stimuli were displayed on a Viewsonic 19"' Perfect Flat monitor using a resolution of $1024 * 768$ pixels and $100 \mathrm{~Hz}$ refresh rate. The experiment was controlled by Presentation software (Neurobehavioral Systems, www.neurobs.com). The monitor was placed in $67 \mathrm{~cm}$ viewing distance. Eye movements were recorded with an Eyelink 1000 eyetracker (SR-Research, binocular mode, $1000 \mathrm{~Hz}$ temporal resolution). Synchronization of the experiment software and the eye tracking device was realized using "The Presentation Extension for the EyeLink Eyetracking System" (Prexel, http://www.cs.umb.edu/ marc/prexel/). Calibration of the eye tracking device was done before the experiments and in breaks if necessary.

Analyses. Trials on which eye movements occurred were excluded from analyses to ensure that effects of the primes were due to spatial attention and not due to eye movements. For each trial we computed a baseline position for each eye averaging the position over 100 ms (100 samples) before prime onset. Participants were instructed to keep their eyes on the fixation cross at the center of the screen during this and the following period of time. Relative to this baseline we computed shifts in gaze position in the time window from prime onset to target offset. Trials on which relative gaze position deviated from the baseline position more than $0.56^{\circ}$ of visual angle were excluded from the analyses of response times and error rates. Every trial that had at least one sample in which eye position data was missing (blink) in the critical time period from prime onset to target offset was excluded as well. Note that the critical time period increases with increasing prime target SOA. Therefore, the probability of eye movement errors increases with SOA, due to the increasing length of the critical period and a correspondingly increasing probability of drifts away from baseline position.

We applied signal-detection analyses and computed $d^{\prime}$ as a measure of sensitivity and $c$ as a measure of response criterion for each participant in each condition (Macmillan \& 
Creelman, 1991). RTs were averaged for correct responses in each condition with post-error trials being excluded. Rate of eye movement errors (including blinks) among trials in each condition was arc-sine transformed. These dependent variables were analyzed with repeated measures ANOVAs with factors Congruency and SOA. In addition we used planned paired ttests comparing $d^{\prime}$, hit rate, and false alarm rate between congruent trials and incongruent trials at each SOA to assess the magnitude of priming effects and the relative contributions of effects on hits and false alarms for single levels of SOA. Prime recognition performance was determined in terms of $d$ ' for each mask separately and then averaged across the two different masks (Albrecht \& Mattler, 2012). We used t-tests to test whether prime recognition performance in terms of d' differed from zero with any SOA. To test whether these measures of $d$ ' differed between levels of SOA, we conducted a repeated measures ANOVA with SOA as independent variable. In the recognition session, eye movements were measured as before but no trials were excluded because we did not expect any effect of eye movements on performance measures. Note, results are presented in the perspective of prime-cue SOA but we keep in mind that this is confounded with cue-target SOA.

\subsubsection{Results}

Sensitivity. Target detection performance (see Figure 3.2A) was better on congruent trials $\left(d^{\prime}=1.76\right)$ than on incongruent trials $\left(d^{\prime}=1.66\right), F(1,11)=18.1, M S e=0.014, p=.001$ and decreased with increasing $\operatorname{SOA}, F(3,33)=6.7, M S e=0.053, p=.005$, with $d^{\prime}=1.86$, 1.76, 1.60, and 1.62 with $24 \mathrm{~ms}, 35 \mathrm{~ms}, 71 \mathrm{~ms}$, and $82 \mathrm{~ms}$ SOA, respectively. The interaction Congruency x SOA was also significant, $F(3,33)=6.2, M S e=0.026, p=.004$. Performance on incongruent trials decreased with increasing $\mathrm{SOA}, F(3,33)=14.9, \mathrm{MS} e=0.033, p<.001$, whereas performance on congruent trials was unaffected by $\operatorname{SOA} F(3,33)=0.62, M S e=$ $0.045, p=.540$ 
A

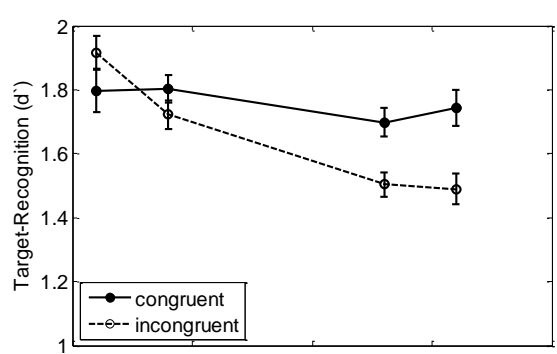

B
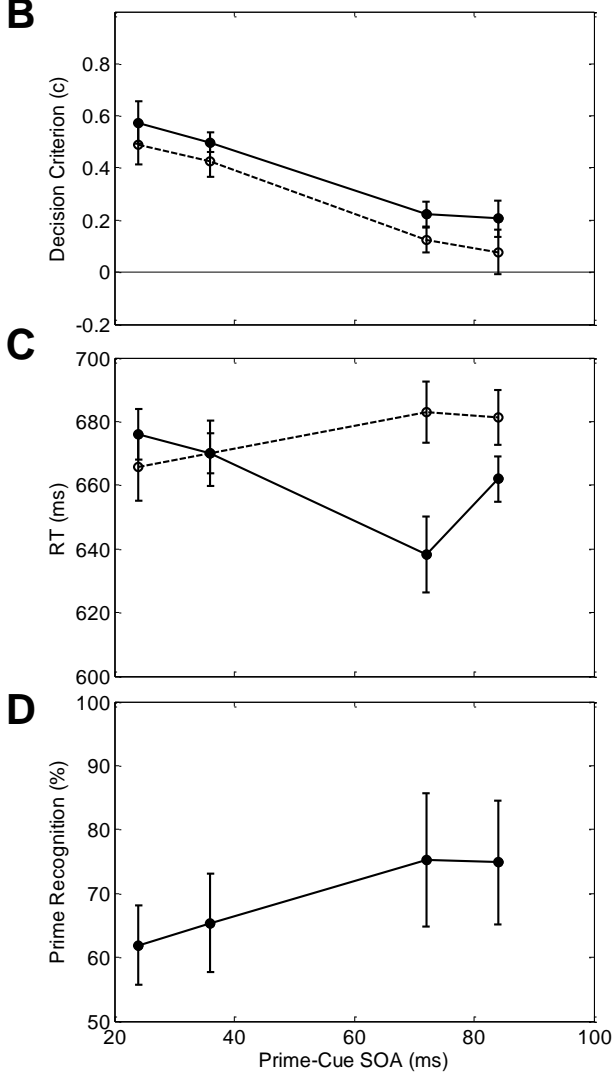

Figure 3.2 Results in Experiment 4. (A) $d$ ' as a measure of sensitivity for congruent and incongruent trials as a function of prime-cue-SOA. Error bars show 95\% within-subject confidence intervals (Loftus \& Masson, 1994). (B) $c$ as a measure of response criterion for congruent and incongruent trials as a function of prime-cue-SOA. Error bars show 95\% within-subject confidence intervals. (C) Response times for congruent and incongruent trials as a function of prime-cue-SOA. Error bars show 95\% within-subject confidence intervals. (D) Prime recognition performance in percent correct as a function of prime-cue-SOA. Error bars show $95 \%$ confidence intervals on mean recognition performance.

To further investigate this interaction we defined the priming effect at each level of SOA as $d^{\prime}$ on congruent trials minus $d^{\prime}$ on incongruent trials. This priming effect differed significantly from zero with the two longer SOAs (71 ms: $d^{\prime}$-effect $=0.19, t=2.8 p=.017$; 
Table 3.1 Mean hit rates and false alarm rates and results of t-tests in Experiment 4.

\begin{tabular}{|c|c|c|c|c|}
\hline & \multicolumn{4}{|c|}{ Prime-Cue SOA (ms) } \\
\hline & 24 & 35 & 71 & 82 \\
\hline & \multicolumn{4}{|c|}{ hit rate $(\%)$} \\
\hline congruent & 61.1 & 63.1 & 68.5 & 68.7 \\
\hline incongruent & 65.7 & 64.9 & 68.5 & 68.2 \\
\hline $\mathrm{t}(11)$ & -2.3 & -1.1 & 0.01 & 0.3 \\
\hline \multirow[t]{2}{*}{$\mathrm{p}$} & .040 & .290 & .990 & .772 \\
\hline & \multicolumn{4}{|c|}{ false alarm rate $(\%)$} \\
\hline congruent & 10.1 & 11.5 & 20.5 & 20.6 \\
\hline incongruent & 11.0 & 13.1 & 24.0 & 26.0 \\
\hline $\mathrm{t}(11)$ & -1.0 & -1.4 & -2.5 & -2.6 \\
\hline $\mathrm{p}$ & 0.337 & 0.184 & 0.032 & 0.025 \\
\hline
\end{tabular}

$82 \mathrm{~ms}$ : $d$ '-effect $=0.25, t=3.9, p=.002)$ and a non-significant trend in the opposite direction with a $24 \mathrm{~ms} \mathrm{SOA}\left(d^{\prime}\right.$-effect $\left.=-0.12, t=-2, p=.069\right)$. There was no significant effect with 35 $\operatorname{ms~SOA}\left(d^{\prime}\right.$-effect $\left.=0.08, t=1.5, p=.158\right)$.

t-tests for hit rates and false alarm rates (see Table 3.1) revealed that Congruency affected hit rate and false alarm rate differently at different SOAs. With $24 \mathrm{~ms}$ SOA hit rate was lower on congruent than on incongruent trials but not significantly affected with longer SOAs whereas false alarm rate with 71 and $82 \mathrm{~ms}$ SOA was higher on incongruent than on congruent trials and was unaffected with shorter SOAs. 
Criterion. The decision criterion (see Figure 3.2B) was more conservative on congruent trials $(c=0.38)$ than on incongruent trials $(c=0.28), F(1,11)=18.8, M S e=0.012$, $p=.001$. The main effect of SOA was also significant, reflecting decreasing values of c (less conservative decisions) with increasing $\mathrm{SOA}, F(3,33)=7.8, \mathrm{MSe}=0.121, \mathrm{p}=.014$, with $\mathrm{c}$ values of $0.53,0.46,0.17$, and 0.14 for $24 \mathrm{~ms}, 35 \mathrm{~ms}, 71 \mathrm{~ms}$, and $82 \mathrm{~ms}$ SOA, respectively. The interaction of SOA and Congruency did not reach significance, $\mathrm{F}(3,33)=0.2$, MSe $=$ $0.018, \mathrm{p}=.901$

$R T$. There was a marginally significant main effect of Congruency on RT (see Figure 3.2C), $F(1,11)=4.4, M S e=993, p=.061$, which was qualified by an SOA x Congruency interaction $F(3,33)=3.0, M S e=1174, p=.045$. In order to better understand this interaction we averaged RT for the two short SOAs and the two long SOAs. Separate t-tests revealed that with short SOAs there was no significant difference between congruent trials $(673 \mathrm{~ms})$ and incongruent trials $(668 \mathrm{~ms}), t(11)=-0.7, p=.474$, whereas with long SOAs RT was shorter on congruent trials $(650 \mathrm{~ms})$ than on incongruent trials $(682 \mathrm{~ms}), t(11)=2.8, p=.017$.

Prime recognition performance. Overall, participants recognized primes on $69 \%$ of the trials. SOA had a significant main effect on prime recognition performance measured in terms of d' (see Figure 3.2D), $F(3,33),=9.2, M S e=0.249, p=.003$, indicating that recognition performance was better with the two longer SOAs compared to the two shorter SOAs. Primes could be discriminated better than chance level at all SOAs $\left(d^{\prime}>0.8, t>3.7, p<.004\right.$ in all cases). To assess the relation of priming effects and prime visibility we calculated the correlation between the two variables for each SOA. There was no significant correlation between the priming effects and prime visibility at any SOA $(r=.17, .21,-.06$, and .08 for 24 ms, $35 \mathrm{~ms}, 71 \mathrm{~ms}$, and $82 \mathrm{~ms}$ SOA, respectively). Scatterplots for these correlations are given in Figure 3.3. 
A
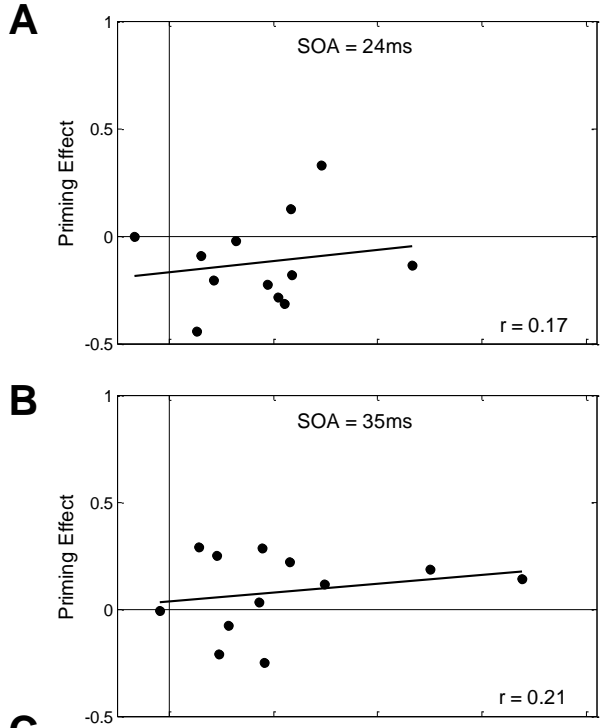

C
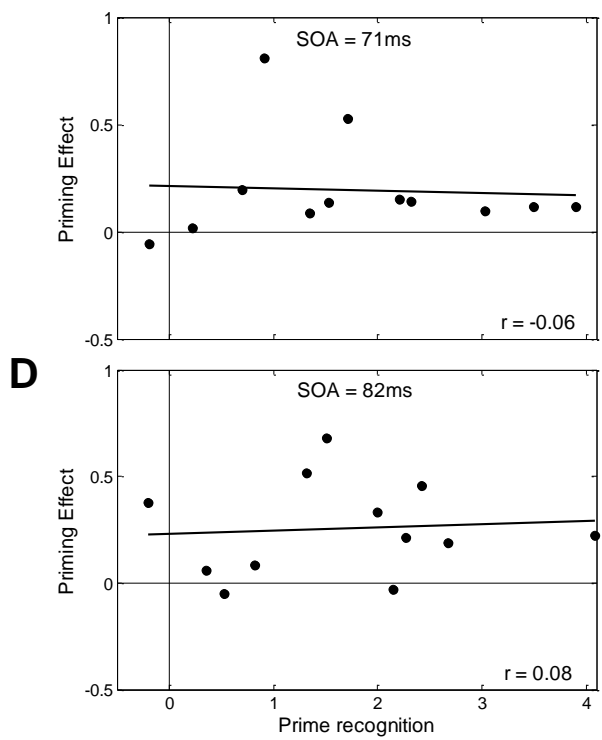

Figure 3.3 Scatterplots with regression lines of priming effects on d' (defined as d' on congruent trials minus d' on incongruent trials) against prime recognition performance with each SOA. Pearson's $r$ is given in the bottom right corner.

\subsubsection{Discussion}

We found an effect of masked primes on detection performance with luminance targets with better performance on congruent than on incongruent trials. This finding suggests that masked primes can affect spatial attention at early levels of target processing. This effect exhibits similar characteristics as priming effects in a previous spatial cueing study where we 
employed a speeded choice-reaction-time task with distractor stimuli (Project 1) and found priming effects increase with increasing prime-cue SOA but decreases with increasing cuetarget SOA. In the present study, however, prime-cue SOA and cue-target SOA were confounded in such a way that both contribute to the priming effect if we assume the same time-course as in the previous study. Thus, the present data does not allow any distinction between the effects of the two types of SOAs.

Cueing effects on measures of response bias in spatial cueing paradigms with signal detection tasks have been explained in the perspective of late selection theories by assuming that decision criteria are more lenient for cued locations if they are more likely to contain targets than other locations (e.g., Duncan, 1980). Hawkins et al. (1990) argued this should result in a more lenient response criterion when the target appears at the validly cued location than on the un-cued location. This view predicts for the present cue-priming approach that the response criterion should be more lenient on congruent than on incongruent trials if priming effects arise from processing at decision levels. In contrast, however, we found that response criteria were more conservative on congruent than on incongruent trials. In other words, participants reported seeing a target less frequently on congruent than on incongruent trials. This finding corresponds to those of Rahnev and colleagues (2011) who reported that attention can induce a conservative bias in perceptual decision tasks. These authors explained this finding within the framework of signal detection theory by assuming that attention reduces trial by trial variability of signal strength, which corresponds to an early selection perspective. According to this view, in our experiment primes modulated early signal quality by focussing attention on the target location on congruent trials to a greater extent than on incongruent trials. In this perspective, however, it remains to be explained why SOA did not modulate the priming effect on criterion. 
An underlying assumption in our calculation of d' from hit rates and false alarm rates is that noise and signal plus noise distributions have the same variance. However, it has been suggested by a reviewer that variance may be larger for target present than for target absent distributions (Swets, 1986) if there is any variation in the signal. This means that, using our calculation, shifts in criterion may lead to changes in $d$ ' because relation of hit rate and false alarm rate is different at different points of the curve. This might mean that the difference in $d$ ' between congruent and incongruent trials does not reflect a change in sensitivity but instead primes solely affect the decision criterion. With the present data we cannot rule out that our analyses are affected by inequality of variances. Nevertheless, upon closer examination, it seems unlikely that this explanation can account for the priming effects on $d$ ' in the present experiments due to the following reasons. First, if the congruent and incongruent conditions represent different points on the same ROC curve and if d' only differs between congruent and incongruent trials as a result of different decision criteria and unequal variances both false alarm rate and hit rate should differ. However, with long SOAs, where priming effects were largest hit rates did not differ between congruent and incongruent trials and only false alarm rates were affected by congruency. This suggests that sensitivity is indeed higher on congruent trials than on incongruent trials (Macmillan \& Creelman, 1991). Second, priming effects on $c$ and $d^{\prime}$ are not affected in the same way by manipulation of SOA. Whereas the priming effect on $d$ ' increases with prime-cue SOA the priming effect on $c$ remains constant. In order to explain priming effects on $d$ ' as resulting from a combination of priming effects on criterion and inequality of variance in the target present and target absent distributions one would have to assume that the variances become more unequal with increasing prime-cue SOA. We believe it is unlikely that manipulation of SOA yields such an effect on signal strength although it may be construed that a reduction in cue-target SOA affects target signal distributions because it decreases the amount of spatial attention to the 
target. Finally, in a similar experiment Luck et al. (1994) analyzed effects of cue validity on sensitivity using both a similar calculation of d' as us as well as the area under the ROC curve and obtained similar results with both measures although there were validity effects on decision criterion.

The typical priming effects on RT were also found as a function of SOA (see Project 1). However, it is difficult to interpret these effects because here we prioritized accuracy over speed in the instruction and there was no emphasis on respond speed.

Our masking procedure reduced prime visibility to an average prime recognition performance of only $69 \%$ correct. However, we did not achieve our goal to reduce prime recognition performance to chance levels. Nonetheless, we did not find any correlation between prime visibility and priming effects on target detection performance. This finding suggests that the priming effect on target detection performance was not modulated by the visibility of the primes. Note that this does not exclude the possibility that priming effects require a minimal amount of prime visibility.

Priming effects on target detection performance and response bias suggest that primes modulate early target processing in our cue-priming paradigm. On the one hand, this might result from a post-central effect of primes which might shift attention like visible cues and enhance signal strength at early levels of processing. On the other hand, however, priming effects of Experiment 4 might also arise indirectly because primes only affect the processing of the cue without any further effect on shifts of spatial attention. To investigate the latter alternative, we extended the cue-priming paradigm by introducing perceptually similar and dissimilar congruent prime-cue pairs in addition to the dissimilar incongruent pairs (Mattler, 2006; Project 1). When this paradigm was used in choice-reaction time tasks, participants responded faster on congruent trials with perceptually similar prime-cue pairs than on 
dissimilar-congruent trials, indicating that perceptual similarity between prime and cues improves performance. However, RTs were still shorter on dissimilar-congruent trials than on incongruent trials, indicating that priming effects are only partially based on perceptual similarity but also extent to post-perceptual levels of processing. To examine this issue in the present paradigm, we conducted Experiment 5.

\subsubsection{Experiment 5}

In Experiment 5 we employed similar timing parameters as in Experiment 4 but varied the perceptual similarity between prime and cue stimuli by using a total of 4 different spatial cues, 2 were mapped to each side, similar to a previous experiment (Project 1, Experiment 3).

\subsubsection{Method}

Participants. 19 new participants (14 women; age $18-26, \mathrm{M}=22.4$ ) were tested in 3 sessions. One additional participant was excluded after poor performance in the practice session (more than $65 \%$ errors).

Tasks. Participants had to perform the same two tasks as in Experiment 4 with the difference that in the present experiment two different cue symbols were used which indicated to attend the left target field and two symbols indicated to attend the right target field. In the prime recognition task, the two symbols that indicated to attend to one side were mapped to one response key and the other two symbols to the other key. In other words, participants had to report whether the presented prime was a symbol that was associated with attending the left or with attending the right target field. This was done to ensure that the recognition test measured the visibility of those stimulus features that potentially generated the cue-priming effects in the target detection session (Schmidt \& Vorberg, 2006). 
A

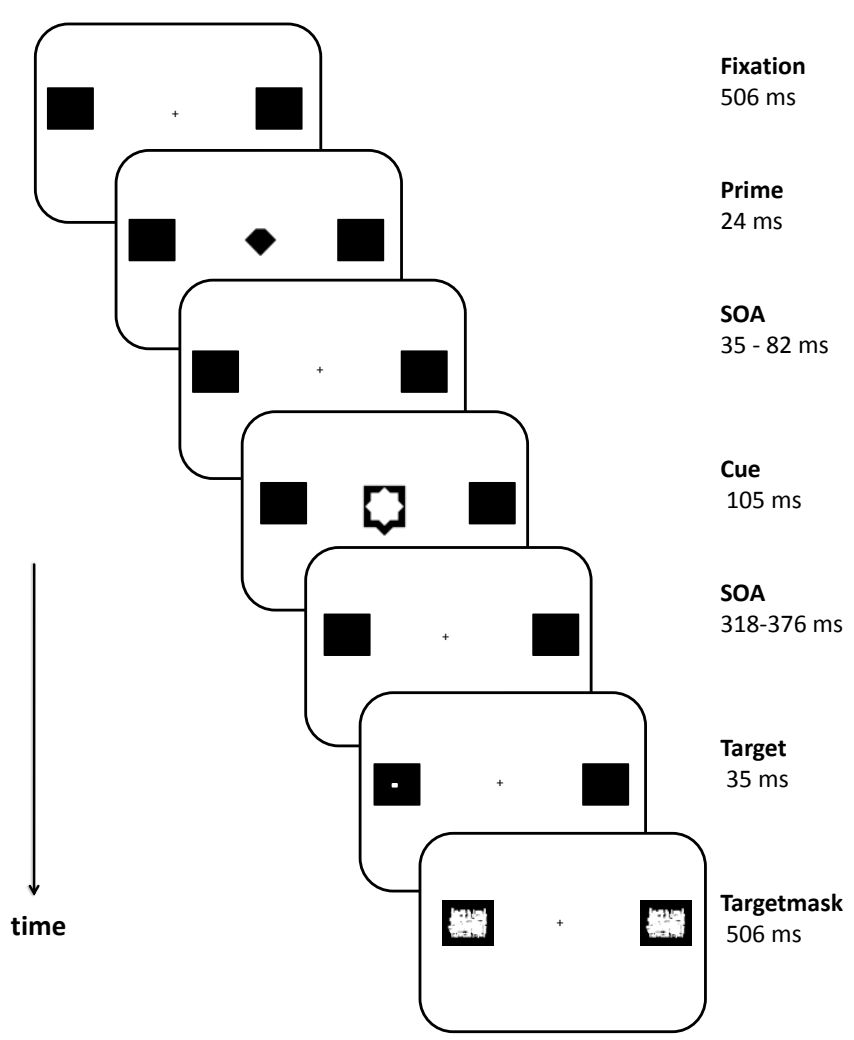

B
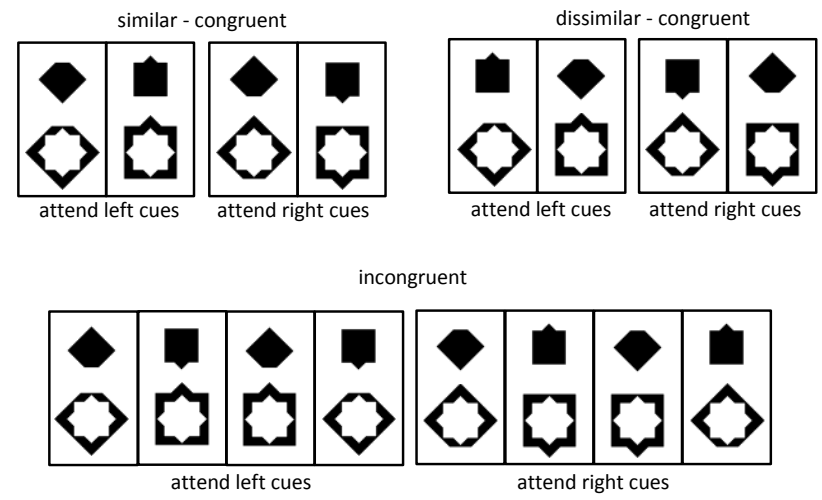

Figure 3.4 Stimuli in Experiment 5. (A) Sequence of events in a trial. (B) Possible combinations of prime and cue stimuli in the three conditions.

Stimuli. Figure 3.4 gives an overview of the stimuli used in Experiment 5 which were variants of the primes and cues used in Experiment 4. Four different stimuli were made out of the diamond and square shaped stimuli of Experiment 4 by cutting off the top or bottom edge of the diamond stimuli and adding one of these edges on the top or bottom of square stimuli. 
This procedure warranted that primes still fitted inside the cut-outs in the cue stimuli. A square with an extension pointing upwards and a diamond with the top part removed predicted a target on the left side, whereas the other two symbols predicted a target on the right side. The sixteen possible combinations of prime-cue pairs can be separated into three categories: (1) the outer shape of primes and cues are replicates of each other (similarcongruent condition, four combinations), (2) primes and cues have different outer shapes but nonetheless indicate to attend to the same side (dissimilar-congruent condition, four combinations), and (3) primes and cues have different shapes and indicated to attend to different sides (incongruent condition, eight combinations). Target stimuli and target masks were the same as those in the previous experiment. Target contrast was adapted to participants performance in the same way as in Experiment 4. As before prime-target SOA was held constant at $400 \mathrm{~ms}$. Prime-cue SOA varied in three steps $(35 \mathrm{~ms}, 59 \mathrm{~ms}$, and $82 \mathrm{~ms})$. In consequence, cue-target SOA was confounded with prime-cue SOA.

Apparatus. The setup and eye movement measurements were the same as in Experiment 4, and the same thresholds were used for trial exclusion.

Design and procedure. As in Experiment 4, participants took part in 3 sessions, a practice session (1h), a long priming session (3-4 h) and a final prime recognition session. In Experiment 5 the independent variable Congruency varied on three levels (similar-congruent, dissimilar-congruent, and incongruent) and each condition was presented on one third of trials. In consequence, incongruent trials occurred on only half as many trials as congruent trials. In order to evenly distribute trials among conditions the total number of trials was slightly changed. The priming session comprised a short practice phase (60 trials) followed by 2592 trials which were separated by longer obligatory breaks into 4 segments of either 720 or 576 trials. Short breaks were introduced after every block of 48 trials. The prime recognition 
session also contained a short practice phase with clearly visible primes and 720 experimental trials. Across 144 trials (three blocks) each of the 8 incongruent prime-cue pairs was presented once and each of the similar-congruent and dissimilar-congruent prime-cue pairs occurred twice on each level of the 3 SOAs and the 2 Targets (absent, present).

Analyses. Dependent measures were analyzed the same way as in Experiment 4 . We defined the perceptual priming effect as the performance difference between similarcongruent and dissimilar-congruent trials because these conditions differ with regard to perceptual similarity but not in congruency regarding which side has to be attended. The postperceptual priming effect was defined as performance difference between dissimilarcongruent and incongruent trials because on these trials prime and cue stimuli were dissimilar but the prime was associated with the same side as the cue on dissimilar congruent trials and with different sides on incongruent trials. Thus, we performed separate ANOVAs to assess both the perceptual and post-perceptual components of the priming effect comparing dissimilar-congruent trials to similar-congruent trials on the one hand and to incongruent trials on the other hand. Again, we used planned paired t-tests to assess the magnitude of both the perceptual and the non-perceptual component at each level of SOA. Measures of d' for prime recognition performance was computed separately for each mask and then averaged across the four masks for each SOA. Recognition performance was analyzed using t-tests for each SOA (to assess whether recognition performance differed from chance level) and also with a repeated measures ANOVA with the independent variable SOA. Note, that we report effects of SOA in the perspective of the prime-cue SOA but keep in mind that this SOA is confounded with the cue-target SOA. 


\subsubsection{Results}

Sensitivity. The main effect of SOA was significant indicating that target detection performance again decreased with increasing SOA (from $d^{\prime}=1.93$ to 1.69 ), $F(2,36)=17.2$, $M S e=0.047, p<.001$. The main effect of Congruency was significant, $F(2,36)=3.3, M S e=$ 0.061, $p=.048$. The interaction Congruency x SOA did not reach significance, $F(4,72)<1$, $p>$.6. The comparison of similar-congruent and dissimilar-congruent trials revealed a marginal significant main effect reflecting a perceptual priming-effect on measures of sensitivity, $d$ '-difference $=0.09, F(1,18)=3.9, M S e=0.056, p=.064$. The comparison of dissimilar-congruent and incongruent trials revealed no significant main effect, indicating the absence of a post-perceptual priming effect, $d^{\prime}$-difference $=0.03, F(1,18)<1, p>.6$. In planned t-tests comparing dissimilar-congruent trials to similar-congruent trials on the one hand and to incongruent trials on the other hand with each SOA the only significant difference was found with $82 \mathrm{~ms}$ SOA between similar-congruent trials and dissimilarcongruent trials, $d^{\prime}$-difference $=0.14, t(18)=2.1, p=.046\left(d^{\prime}\right.$-difference $<0.12 ; t(18)<1.4, p$ $>.19$, in all other cases).

t-tests of hit rate and false alarm rate (see Table 3.2) revealed that with $82 \mathrm{~ms}$ false alarm rates which were marginally increased on dissimilar-congruent trials compared to similar-congruent trials whereas with $59 \mathrm{~ms}$ SOA both hit rate and false alarm rate were increased on dissimilar-congruent trials compared to similar-congruent trials. There were no significant post-perceptual priming effects on either hit rate or false alarm rate. 
A

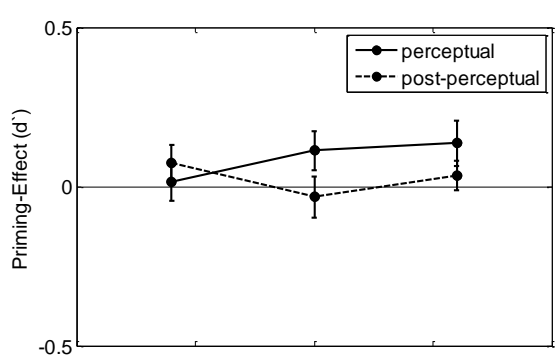

B
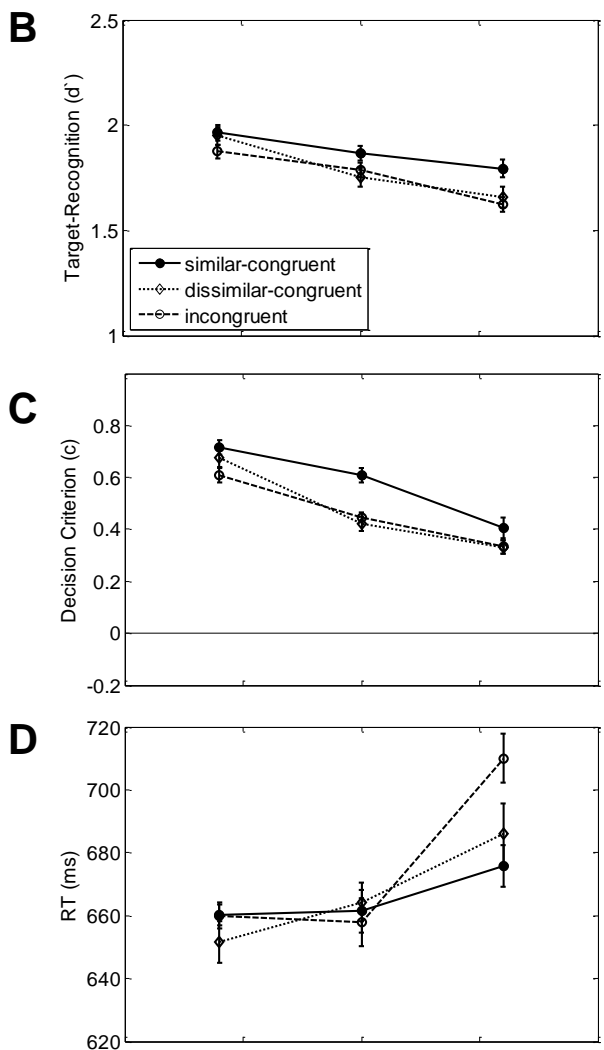

E

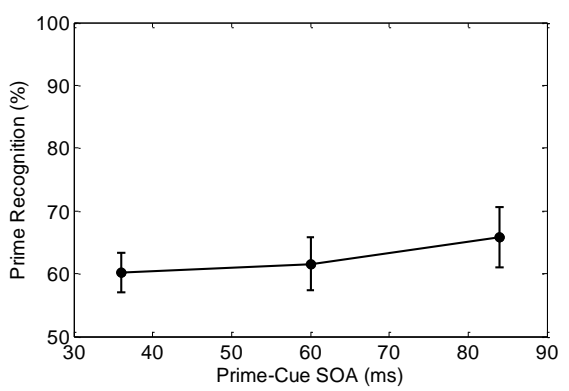

Figure 3.5 Results in Experiment 5. (A) Priming effect on d' (perceptual priming effect is defined as d' on similar-congruent trials minus d' on dissimilar-congruent trials, non-perceptual priming effect is defined as d' on dissimilar-congruent trials minus d' on incongruent trials). (B) $d^{\prime}$ 'as a measure of sensitivity for similar, congruent and incongruent trials as a function of prime-cue-SOA. Error bars show 95\% within-subject confidence intervals. (C) $c$ as a measure of response criterion for similar, congruent and incongruent trials as a function of prime-cue-SOA. Error bars show 95\% within-subject confidence intervals. (D) Response times for similar-congruent, dissimilar-congruent and incongruent trials as a function of prime-cue-SOA. Error bars show 95\% within-subject confidence intervals. (E) Prime recognition performance in percent correct as a function of prime-cue-SOA. Error bars show 95\% confidence intervals of mean recognition performance. 
Criterion. Participants responded more conservatively with short prime-cue SOA, as indicated by the significant main effect of SOA, $F(1,18)=39.3, M S e=0.035, p<.001$, with $c=0.67,0.49$, and 0.36 with $24 \mathrm{~ms}, 59 \mathrm{~ms}$, and $82 \mathrm{~ms} \mathrm{SOA}$, respectively. The main effect of Congruency was also significant, $F(1,18)=6.8, M S e=0.032, p=.003$. The comparison of similar-congruent and dissimilar-congruent trials revealed a significant effect reflecting a perceptual priming effect, $F(1,18)=7.5, M S e=0.038, p=.013$, whereas the comparison of dissimilar-congruent and incongruent trials was not significant, $F(1,18)<1, p>.6$. Thus, participants responded more conservatively on similar-congruent trials $(c=0.58)$ than on dissimilar-congruent trials $(c=0.48)$ and incongruent trials $(c=0.46)$. The interaction Congruency $\mathrm{x}$ SOA did not reach significance, $F(4,72)=1.8, M S e=0.018, p=.156$

$R T$. The main effect of SOA was significant, $F(2,36)=11.3, M S e=1665, p<.001$, indicating that RTs increased with increasing SOA from $657 \mathrm{~ms}$ to $691 \mathrm{~ms}$. The main effect of Congruency did not reach significance, $F(2,36)=1.7, \quad p=.191$, and the interaction Congruency x SOA was only marginally significant, $F(4,72)=2.2, M S e=1107, p=.080$. With $82 \mathrm{~ms}$ SOA we expected the largest priming effects. Separate tests of these trials revealed a significantly shorter RTs on dissimilar-congruent trials (686 ms) than on incongruent trials $(710 \mathrm{~ms}), t(18)=2.1, p=.033$. RT on similar-congruent trials $(676 \mathrm{~ms})$, however, did not differ significantly from RT on dissimilar-congruent trials $(t<1, p>.4)$. There was no other significant priming effect on RT with the two shorter SOAs $(t<1, p>.4$ in all cases).

Prime recognition performance. Prime recognition performance was modulated by SOA as reflected by the significant main effect of SOA, $F(2,36)=16, M S e=0.099, p<.001$, with d' values of $0.15,0.35$, and 0.72 for $35 \mathrm{~ms}, 59 \mathrm{~ms}$, and $82 \mathrm{~ms}$ SOA, respectively. Prime recognition performance differed significantly from zero with SOAs of $59 \mathrm{~ms}$ and $82 \mathrm{~ms}$ 
Table 3.2 Mean hit rates and false alarm rates and results of t-tests in Experiment 5.

\begin{tabular}{|c|c|c|c|}
\hline & \multicolumn{3}{|c|}{ Prime-Cue SOA (ms) } \\
\hline & 24 & 59 & 82 \\
\hline & \multicolumn{3}{|c|}{ hit rate $(\%)$} \\
\hline congruent-similar & 60.0 & 62.0 & 67.7 \\
\hline congruent-dissimilar & 61.0 & 65.8 & 67.9 \\
\hline incongruent & 62.0 & 66.0 & 66.8 \\
\hline $\mathrm{t}_{\text {perceptual }}(18)$ & -0.9 & -2.1 & -0.1 \\
\hline $\mathrm{p}_{\text {perceptual }}$ & .383 & .048 & .893 \\
\hline$t_{\text {postperceptual }}(18)$ & -0.9 & -0.1 & 1.0 \\
\hline \multirow[t]{2}{*}{ Ppostperceptual } & .390 & .919 & .346 \\
\hline & \multicolumn{3}{|c|}{ FA rate $(\%)$} \\
\hline congruent-similar & 7.3 & 8.9 & 12.9 \\
\hline congruent-dissimilar & 7.0 & 12.6 & 15.6 \\
\hline incongruent & 8.3 & 12.0 & 15.8 \\
\hline $\mathrm{t}_{\text {perceptual }}(18)$ & 0.3 & -2.8 & -2.1 \\
\hline$p_{\text {perceptual }}$ & .765 & .012 & .053 \\
\hline $\mathrm{t}_{\text {postperceptual }}(18)$ & -1.5 & 0.5 & 0.3 \\
\hline $\mathrm{p}_{\text {postperceptual }}$ & .142 & .597 & .761 \\
\hline
\end{tabular}

SOA, $\mathrm{t}(18)=2.7, p=.015$, and $t(18)=4.2, p<.001$, respectively, but not with $35 \mathrm{~ms}$ SOA, $t(18)=2.1, p=.055$. 


\subsubsection{Discussion}

Experiment 5 replicated Experiment 4 showing cue-priming effects in a signal detection task. Participants' target detection performance was improved on trials with similarcongruent primes compared to trials with incongruent primes. It has been repeatedly found that cue-priming effects increase with prime-cue SOA (e.g., Mattler, 2003; Project 1). Planned contrasts confirmed for the present experiment the expected larger priming effects with longer prime-cue SOA. Moreover, participants responded more conservatively on congruent than on incongruent trials. With increased prime-cue SOA, sensitivity and the conservative response bias decreased. This can be rephrased because prime-cue SOA was confounded with cue-target SOA: with increased cue-target SOA sensitivity and the conservative response bias increased.

There was no difference in detection performance, however, between trials with congruent but perceptually dissimilar primes and those with incongruent primes. This finding suggests that cue-priming effects in the present luminance detection task crucially depended on perceptual similarity of primes and cues. This is supported by planned contrasts which revealed a significant difference between similar-congruent and dissimilar congruent trials. This pattern of results indicates that priming effects on detection performance result from repetition priming at perceptual levels of cue processing. Although prime and cue stimuli were not identical because they differed in size and the cut-outs of the cues, their task relevant outer shape was the same which might have facilitated perceptual processing of the cues. One way to conceive the effect of perceptual priming of cues and the priming effects on target detection performance is to assume that more rapid cue processing might leave more time to shift attention to the target location. 
The only apparent post-perceptual priming effect was found on RT with $82 \mathrm{~ms}$ SOA. As mentioned above, however, interpretation of RT data in the present target detection task requires caution, because participants were instructed to use as much time as they wished to select their response. Nevertheless, this effect corresponds to post-perceptual effects on RT that we found in a previous choice RT experiment (Project 1). However, RT effects can be explained without assuming priming effects on early levels of target processing. For instance, the RT effect might as well result because a decision is reached sooner on congruent trials than on incongruent trials without any effect on target signal enhancement.

\subsubsection{General Discussion}

In two experiments, we found effects of masked primes on detection performance of masked luminance targets in terms of measures of sensitivity and response criterion. On the one hand, these effects exceed previous priming effects found in other cue-priming studies indicating priming effects in terms of modulated target processing at earlier perceptual levels. On the other hand, however, these priming effects were only found with perceptually similar prime-cue pairs indicating that cue-priming effects on early target processing result from their effect on the processing of the cue. The absence of cue-priming effects with perceptually dissimilar primes suggests the absence of direct cue-priming effects on early levels of target processing. On the background of the post-perceptual cue-priming effects in our previous study (Project 1), we surmise that the absence of post-perceptual effects in the present study result from differences between the previous choice RT task and the present target detection task. We speculate that the previously found post-perceptual effects result from post-central levels of processing because the tasks were identical in all other respects. In the following we discuss different aspects of this pattern of results. 


\subsubsection{Scope of spatial cue-priming effects}

Which mechanisms are best suited to explain cue-priming effects on target detection performance? According to early selection theory, one mechanism is sensory amplification or gain control which means that information from attended locations triggers stronger neuronal responses than information from unattended locations early perceptual levels of processing (Hillyard, Vogel \& Luck, 1998). In consequence, signal-to-noise ratio is higher for attended signals. Higher signal quality for an attended location then leads to overall more effective processing which results in better accuracy and faster responses for attended in contrast to unattended stimuli.

According to late selection models, however, attentional effects could be based on uncertainty reduction. According to Shiu \& Pashler (1994) in displays in which information from different positions can be confused with the target cueing effects could result due to a reduction of spatial uncertainty which makes inclusion of information from irrelevant locations less likely. In other words, cueing effects result because noise from irrelevant positions can be excluded from the decision. Spatial uncertainty is introduced by displays in which participants cannot be certain about the location where the target has been presented and they cannot base their response on information from this location. This is especially relevant with masked targets when masks are presented at multiple locations. This problem has been addressed by providing participants with a post-cue which marks the target location (Hawkins et al., 1990, Luck et al. 1994). If only one mask is used it can be used to mark the relevant location as well. Luck and colleagues (1994) used a similar task like in the present experiments and found that cueing effects did not differ between conditions in which either only a single or four possible target locations were masked. However, in these experiments, a post-cue was presented in the four mask condition so that participants were aware of the 
potential target location. Here, we did not employ post-cues because our visible cues were always validly indicating the target location. Therefore, we assume that participants were always certain about the relevant position. In consequence, it seems unlikely that uncertainty reduction plays a crucial role in the present experiments.

Priming effects on sensitivity d' were accompanied by priming effects on decision criterion c indicating that participants applied more conservative criteria - reported seeing a target less frequently - on congruent than on incongruent trials. On the one hand, this criterion effect could reflect that participants were looking on the entire display for some kind of irregularity in the stimulus sequence as a sign for a target presentation, if participants mistook an irregularity at the cue location in the centre of the screen as a sign for the presence of a target which occurred more often on incongruent than on congruent trials. Contrary to this view, however, a similar irregularity at the cued location should have occurred on dissimilarcongruent trials which did not produce a comparable effect on criterion in Experiment 5. Alternatively, the more conservative response bias on congruent trials could be due to an increased spatial uncertainty on incongruent than on congruent trials which could have the consequence that noise from the masks was classified as a target if noise from two instead of just one location was factored in (Shaw, 1984). However, as mentioned above, this view seems to be unlikely due to the $100 \%$ valid cues. Therefore, the most likely account for our criterion effect seems to be that early target processing is modulated by congruent primes in a similar way as by a visible valid cue which directs attention to a location and reduces the trial by trial variability in signal strength on an early level of target processing (Rahnev et al., 2011). According to this view, our priming effects on measures of response criterion provide further evidence for priming effects on early levels of target processing. 


\subsubsection{Source of the present cue-priming effects}

Mattler (2003) distinguished three possible loci where cue-priming effects could arise. Applied to the present experiments, primes could act either on perceptual levels affecting perceptual processing of cues, on central levels modulating a decision to attend to one or the other side, or at post-central levels initiating shifts of attention. Therefore, the present perceptual cue-priming effects could result if congruent primes facilitate cue processing and incongruent primes impair cue processing in consequence of perceptual similarity between primes and cues. Facilitated cue processing could then lead to sooner shifts of attention. As cue-target SOA seems to be critical for the allocation of attentional resources (Luck, Hillyard, Mouloua \& Hawkins, 1996), considerable effects of these perceptual interactions might extend to resource allocation and thus to detection performance. The finding that detection performance decreased with prime-cue SOA and therefore increased with cue-target SOA supports the view that the limited time to process the cue is a determining factor of detection performance in the present experiments. Moreover, in both experiments of the present study, congruent primes as compared to incongruent primes had a comparable effect on measures of sensitivity and response criterion like a corresponding increase in cue-target SOA. Therefore, it seems plausible that primes have a primarily facilitative effect on cue processing when prime and cue are perceptually similar or corresponding hindering effects when prime and cue are perceptually different.

Previous studies have made different efforts to examine weather cue-priming effects go beyond priming of cue processing (Mattler, 2006; Project 1, Reuss et al., 2011). In the choice-RT task of Project 1 (Experiment 3) only part of cue-priming effects on spatial attention can be explained as perceptual priming of cue processing because dissimilarcongruent primes facilitated responses as compared to dissimilar-incongruent trials. The 
corresponding effect was absent in Experiment 5 of the present study. Keeping in mind, that the absence of evidence is not evidence of absence we speculate that perceptual priming of cue processing might explain the entire priming effect in the present signal detection experiments. If this would be true, the question arises, why priming effects go beyond perceptual similarity in the choice-RT task but not in the signal-detection task. A simple account would assume that his difference results because cue-priming effects were stronger in the choice-RT task than in the target-detection task. In this case, post-perceptual cue-priming effects would have been obtained also in the target-detection task if conditions were set so that primes have a larger impact. Alternatively, however, there might be more fundamental differences between priming effects in the two types of tasks.

\subsubsection{Difference between cue-priming in signal detection and choice-RT tasks}

Several differences between the present signal-detection task and the previous choice RT task have to be considered to understand why cue-priming effects are absent in the targetdetection task when prime and cue are perceptually dissimilar. A comparison between the paradigm used in the present study and that in our previous study (Project 1) reveals that we used identical stimuli but with slightly different time parameters. More specifically, the cuetarget SOA was longer in the present study than in the previous one. Based on the finding that cue-priming effects decay relatively quickly (Mattler, 2005) one could speculate that postperceptual effects might decay more quickly than the perceptual effects. This view gains some plausibility from the fact that perceptual priming effects arise from the influence of the prime on cue processing which might depend more on prime-cue SOA than on cue-target SOA. If one assumes that post-perceptual priming effects decayed after $400 \mathrm{~ms}$ prime-target SOA it would follow that these effects are absent in the present study. 
The similarity of the two paradigms in terms of prime and cue stimuli suggests that perceptual processing of prime and cue stimuli does not differ in the two tasks. Moreover, central processing of cues should also be comparable in the two tasks because the same cue stimuli were mapped to the same instruction to attend to the same spatial locations in both paradigms. Tasks differ, however, in the processing of the target stimuli. In the choice-RT task two visible letter stimuli are presented at the two potential target locations and participants have to select the letter at the indicated location and determine the motor response that is associated to this letter to execute a rapid response. In the target detection task, in contrast, only one luminance target is presented on half of the trials followed by the presentation of two masks at the two potential target locations. Participants have to evaluate this target-mask complex and decide without speed stress whether they think the target was present or absent. If one assumes that central levels of cue-processing do not depend on the processes that are required for target processing, the absence of non-perceptual priming effects in the present study suggests that the post-perceptual effects of primes in the previous choice RT task result from their effects on post-central levels of processing.

Keeping in mind that the absence of evidence is not evidence for the absence of a postperceptual effect, we add the following speculations about the findings in the signal-detection task. According to current theories of consciousness (Dehaene \& Changeux, 2011; Dennett, 2009; Lau \& Rosenthal, 2011; Tononi, 2004) conscious stimuli differ from unconscious stimuli in respect of their capacity to activate higher levels of processing. Consistent with this idea, we speculate that primes might need a high level of activation to gain access to postcentral top-down processes which modulate early level target processing. In other words, perceptually dissimilar primes might be strong enough to produce an effect at post-central levels in the choice-RT task but their impact might not be sufficiently strong to affect later 
post-central processes that are involved in top-down activation of the target processing pathway down to early levels of target processing. In this case, post-central levels of processing in the target-detection task might constitute one limit of the effects of unconscious stimuli.

\subsubsection{Conclusion}

The present study provides new evidence for cue-priming effects on endogenous shifts of spatial attention. Together with a previous study, the picture emerges that masked stimuli have only limited access to post-perceptual processes. Cue-priming of shifts of spatial attention seems to provide a useful tool to specify these limits of masked stimuli. Future research should combine this paradigm with physiological measures to examine the limits of the effects of masked stimuli which might provide new insights into the function of consciousness.

\subsection{Experiments 6 and 7 - Preconditions for spatial cue-priming in signal detection tasks}

Two additional attempts to study spatial cue-priming effects on signal detection performance with improved masking of primes failed to show any significant priming effects. These experiments, which are reported in the following section were conducted before Experiment 5 but are included here as Experiments 6 and 7 here because they are not part of the submitted manuscript. The original purpose of Experiment 6 was to replicate the priming effects found in Experiment 4 under conditions of improved masking while at the same time investigating the role of cue-target SOA in priming effects on signal detection. Priming effects in Project 1 were found to decrease with increasing cue-target SOA but preliminary results suggested that priming effects on signal detection occur only with longer cue-target 
SOAs. Prime recognition was made more difficult by using arrangements of two stimuli as cues and primes in which location of the critical stimulus varied from trial to trial. In addition, primes were masked by a star shaped stimulus which was found to increase masking in preliminary experiments.

\subsubsection{Experiment 6}

Experiment 6 was designed to replicate the priming effect on signal detection with improved masking and to disentangle the effects of prime-cue SOA and cue-target SOA. To this end, we changed prime and cue stimuli in a way that would reduce prime visibility while still allowing for priming effects and varied cue-target SOA with constant prime-cue SOA. In a letter discrimination task, we found that priming effects seem to be largest at short cuetarget SOAs. In contrast, resource allocation to a location in space seems to develop over time (Luck, Hillyard, Mouloua \& Hawkins, 1996). By using a wide range of cue-target SOAs we aimed to clarify this discrepancy.

\subsubsection{Method}

Participants. 18 new participants (16 women; age 19-27, $\mathrm{M}=21.9$ ) completed 3 sessions of the experiment in exchange for course credit or payment of $€ 42$. All had normal or corrected to normal vision. 4 additional participants were excluded after the practice session because of poor performance. One additional participant was excluded because of a large amount of eye movement errors.

Task. Participants had to perform the same task as in Experiment 4. In Experiment 6 the cue and prime symbols could appear above or below fixation and were accompanied by distractors. 
Stimuli. Stimuli were presented using the same setup as in Experiment 4. In this experiment, we introduced two possible positions for prime and cue stimuli. Primes and cues were the same squares and diamonds as in Experiment 4. The cue was presented above fixation half of the time and below fixation on the remaining trials $\left(2^{\circ}\right.$ from fixation to centre of the symbols). At the opposing position, a distractor, which was an overlay of both the square and the diamond prime, was presented together with the prime. Then the cue was presented at the opposing position together with another distractor at the prime's position (this time an overlay of both cue stimuli).

Thus, primes were always masked by the distractor stimulus and prime and cue were

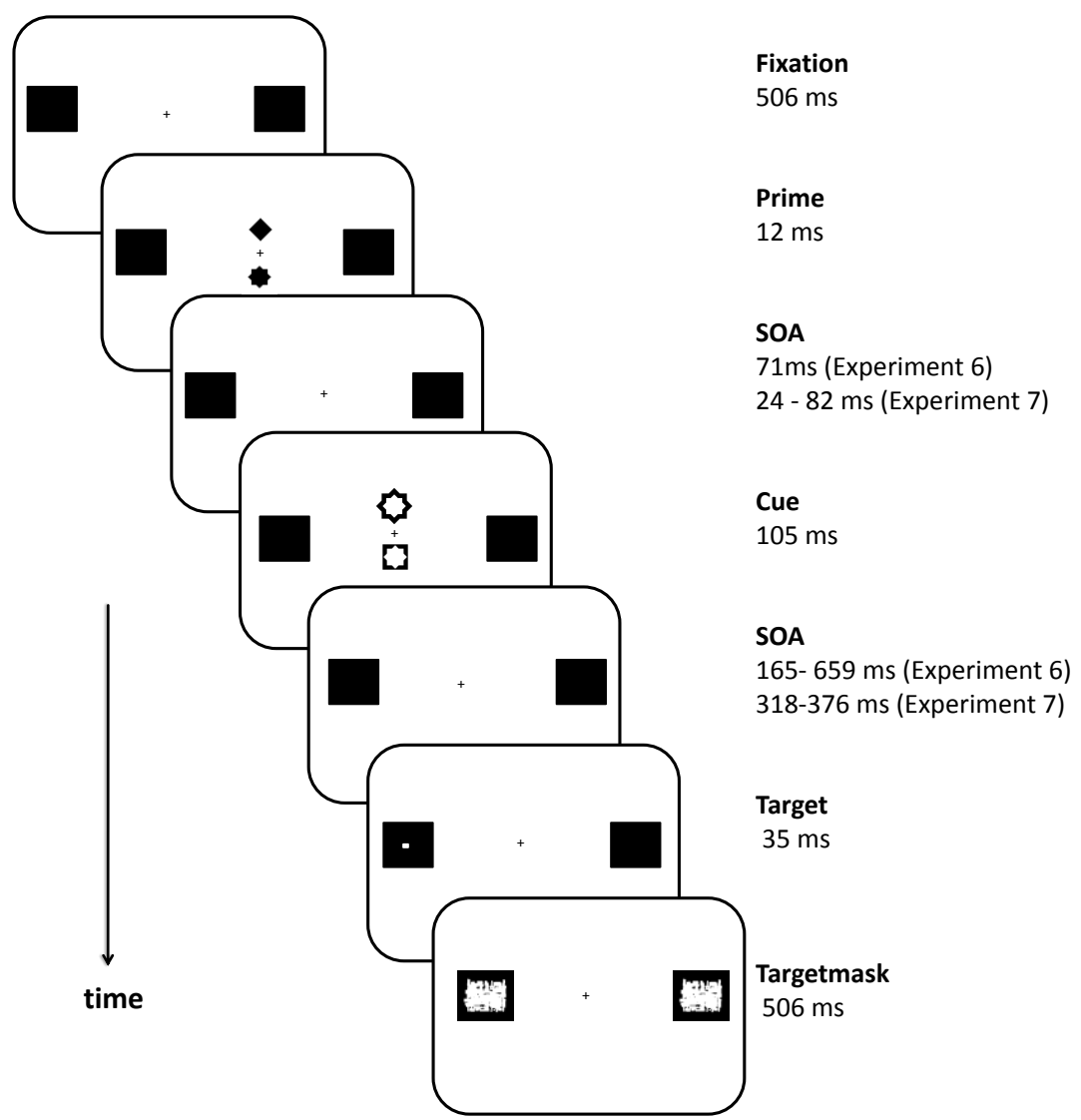

Figure 3.6 Sequence of stimulus events in Experiments 6 and 7. 
presented at different locations. This was done to minimize perceptual interactions between the two stimuli and to increase masking of the prime. Preliminary research suggested that in this particular square or diamond discrimination task, star shaped masks are more effective than square or diamond masks. Furthermore, the added spatial uncertainty should render recognizing the primes more difficult. Targets and target masks were the same as in Experiment 4 and adapted to participants performance in the same way. In Experiment 6 prime-cue SOA was held constant at $72 \mathrm{~ms}$ and cue-target SOA varied in four steps of $165 \mathrm{~ms}$ from 165 to $659 \mathrm{~ms}$. Other timing parameters were the same as in Experiment 4 and are given in Figure 3.6.

Design and procedure. Design and procedure were essentially the same as in Experiment 4 with the difference that the primes could be presented at two different locations. Each possible combination of 2 prime positions, 2 primes, 2 cues, 2 targets and 4 SOAs was presented once in each block. Dependent measures were analyzed the same way as in Experiment 4.

Apparatus. We used the same experimental setup as in Experiment 4.

Analyses. Trials with eye movements were excluded using the same procedure as in Experiment 4 with the exception that thresholds for trials exclusion were increased to $1.12^{\circ}$ visual angle deviation from baseline, because long cue-target SOAs made eye movement errors due to random drift occur very frequently.

\subsubsection{Results}

Sensitivity. Target detection performance is shown in Figure 3.7A. We found that sensitivity increased with cue-target-SOA, $F(3,51)=39.8, M S e=0.094, p<.001$, from $d^{\prime}=1.24$ at 165 ms to 1.93 at $494 \mathrm{~ms}$, but then levelled with 1.92 at $659 \mathrm{~ms}$. Congruency did not have an 


\section{A}

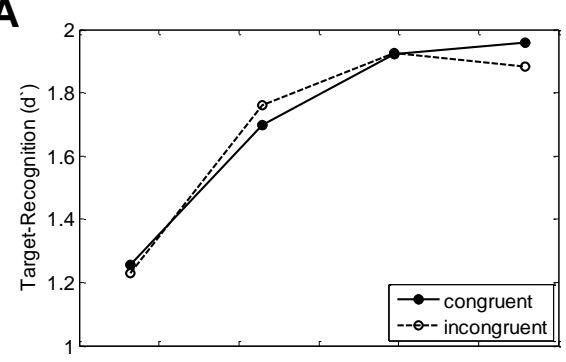

B
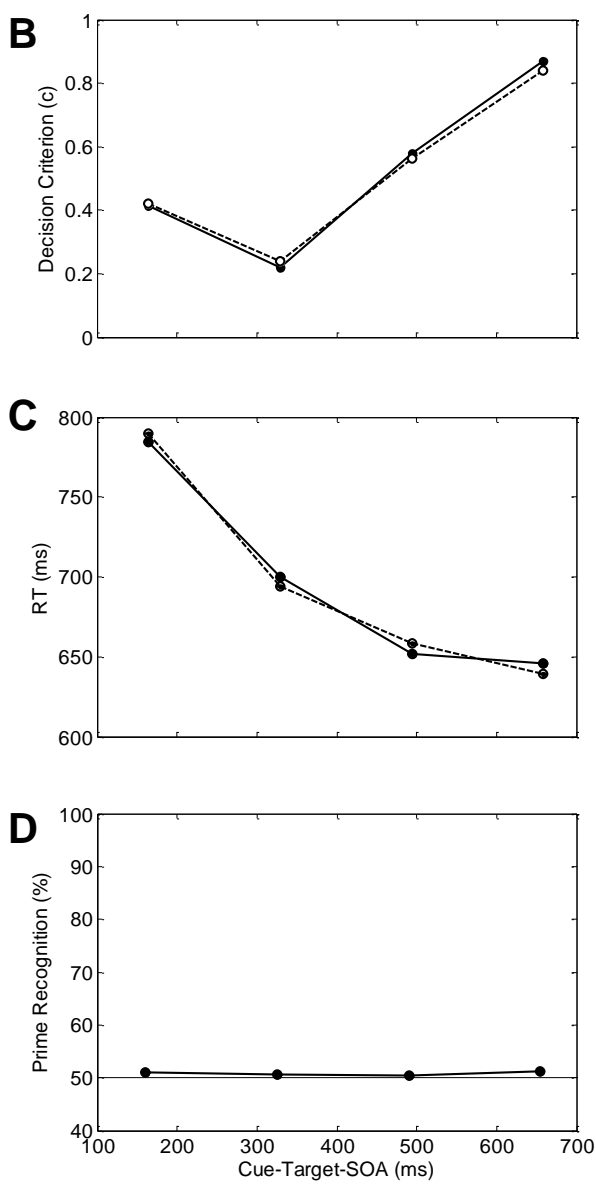

Figure 3.7 Results in Experiment 6. (A) $d$ ' as a measure of sensitivity for congruent and incongruent trials as a function of cue-target-SOA. (B) $c$ as a measure of response criterion for congruent and incongruent trials as a function of cue-target-SOA. (C) Response times for congruent and incongruent trials as a function of cue-targetSOA. (D) Prime recognition performance in percent correct as a function of cue-target-SOA.

effect on target detection, $F(1,17)<0.1, M S e=0.036, p=.772$, nor was there a significant interaction with SOA, $F(3,51)<1, M S e=0.037, p=.464$. 
Criterion. A similar pattern emerged for decision criteria (Figure 3.7B) that were unaffected by congruency, $F(1,17)<0.1, M S e=0.012, p=.798$, but differed at the different SOA levels, $F(3,51=10.7, M S e=0.233, p<.001$. Post-hoc t-tests showed significant differences between the $659 \mathrm{~ms} \mathrm{SOA}$ and all other SOAs $(t(17)>2.7, p<.015$ in all cases) and between $494 \mathrm{~ms}$ and $329 \mathrm{~ms} \mathrm{SOA}, t(17)=5.3, p<.001$. Again, there was no significant interaction of Congruency and SOA, $F(3,51)<1, M S e=0.013, p=.749$.

RT. RT (see Figure 3.7C) decreased with increasing cue-target SOA from $845 \mathrm{~ms}$ at $165 \mathrm{~ms}$ to $686 \mathrm{~ms}$ at $659 \mathrm{~ms}, F(3,51)=61.4, M S e=2500, p<.001$, but was unaffected by congruency, $F(1,17)<0.1, M S e=634, p=.974$. There was no interaction of Congruency and SOA, $F(3,51)<1, M S e=478, p=.419$.

Prime Recognition. Prime recognition performance (see Figure 3.7D) was unaffected by cue-target-SOA, $F(3,51)<0.1, M S e=0.048, p=.925$, and did not differ from chance level (mean $d^{\prime}$ across all SOAs $\left.=0.046, t(17)=1.1, p=0.272\right)$.

\subsubsection{Discussion}

In Experiment 6 prime recognition performance was reduced to chance level. At the same time, we found no effects of primes on performance, as both sensitivity and response bias were unaffected by congruency. As target stimuli were the same as in Experiment 4, changes in prime stimuli or in SOAs have to account for these negative results. Variation of cue-target SOA yielded strong effects on target detection performance, bias and RT. RT difference between the shortest and longest SOA is larger than what would be expected from an attentional modulation of target processing. Thus, slow responses at short cue-target SOAs could result from interference from cue processing. In order to detect targets, participants need to know which side to decide on. Thus they need to have processed the cue stimulus 
which might not be the case at target presentation with short SOAs. Decay of target representations until the cue has been processed could then explain poor recognition performance at short SOAs. Effects of SOA on decision criteria could be explained by reduced trial by trial variability of signal strength through attention at long SOAs (Rahnev et al, 2011).

Several possible explanations for the lack of priming effects on target detection in Experiment 6 can be conceived. The most obvious change from Experiment 4 lies in the more complex prime and cue stimuli. These changes led to better masking as was intended. However, given that prime visibility in Experiment 4 was not associated with priming effects, it seems unlikely that reduced prime visibility is the cause for the absence of priming in Experiment 6. Alternatively, primes might have simply been too weak to affect detection performance, as their critical features were more difficult to discriminate. One reason for this could be spatial uncertainty. As participants did not know at which location the relevant cue symbol would appear, they had to divide their attention across both locations. Naccache, Blandin \& Dehaene (2002) found that temporal attention is a prerequisite for priming effects. If the same is true for spatial attention, then spatial uncertainty could reduce priming effects. However, given that cues could also appear at either location, attention might have been divided, yet should not have been completely absent. Another aspect is that prime duration was reduced from $24 \mathrm{~ms}$ to $12 \mathrm{~ms}$. In addition to reducing prime visibility, shortening prime duration might have reduced the strength of the primes. However, from other studies (Mattler, 2003; Vorberg et al., 2003) it seems that the magnitude of priming effects is largely determined by prime-cue SOA rather than prime duration.

Another possible explanation can be found in the introduction of long cue-target SOAs. In Experiment 6, cue-target-SOA had an effect on target detection performance. This 
suggests that participants were not able to shift their attention completely to the cued location in time for target presentation. Thus, it might be that for priming effects to occur, it is necessary that participants are under stress to quickly shift their attention, either because priming effects are a direct result of accelerated attention shifting in congruent trials compared to incongruent trials or because speed stress is necessary for primes to be sufficiently processed. Either way, the introduction of long cue-target-SOAs might subjectively reduce speed stress in the task and therefore reduce priming effects.

In a second attempt to replicate priming effects on target detection performance we conducted another experiment. As both spatial uncertainty and reduced prime duration might be crucial to reduce prime visibility we kept these changes but otherwise used the same parameters as in Experiment 4.

\subsubsection{Experiment 7}

In Experiment 7 we tried to clarify which changes from Experiment 4 to Experiment 6 were responsible for the vanishing of the priming effect. The most prominent changes were the introduction of long cue-target SOAs and the more complex prime and cue stimuli. Seeing that we successfully reduced prime visibility with the more complex configuration, we used the same SOAs as in Experiment 4 but with the primes and cue configuration from Experiment 6. If priming effects are again absent in this experiment we would conclude that this configuration is too complex to be processed fast enough to allow priming effects. On the other hand, if priming effects emerge it would mean that variations in cue-target SOA can have more profound effects than previously believed and at the same time replicate the effect from Experiment 4 with improved masking. 


\subsubsection{Method}

Participants. 12 new participants (10 women; age 19-32, $\mathrm{M}=24.9)$ were tested in 3 sessions. 3 additional participants were excluded after poor performance in the practice session.

Task. Participants had to perform the same task as in Experiment 6.

Stimuli. Stimuli were presented using the same setup as in the previous experiments. Prime and cue stimuli were the same as in Experiment 6. Targets and target masks were the same as in the previous experiments and adapted to participants performance in the same way. Prime-cue SOA and cue-target SOA varied in the same way as in Experiment 4. However, prime duration was $12 \mathrm{~ms}$ like in Experiment 6. Other timing parameters were the same as in Experiment 4 and are given in Figure 3.6.

Apparatus. The same setup as in the previous experiments was used. Eye movements were measured as in the previous experiments. The same eye movement thresholds as in Experiment 4 were used for trial exclusion.

Design and procedure. Design and procedure were the same as in Experiment 6.

Analyses. Dependent measures were analyzed the same way as in Experiment 6.

\subsubsection{Results}

Sensitivity. Target detection performance (see Figure 3.8A) was unaffected by Congruency, $F(1,11)=0.2, M S e=0.042, p=.635$, but decreased with $\mathrm{SOA}, F(3,33)=7.4$, $M S e=0.041, p=.002$. There was a marginal interaction between the two factors, $F(3,33)=$ 2.4, MSe $=0.048, p=.087$. Paired t-tests evaluating priming effects for each of the 4 SOAs by 
A

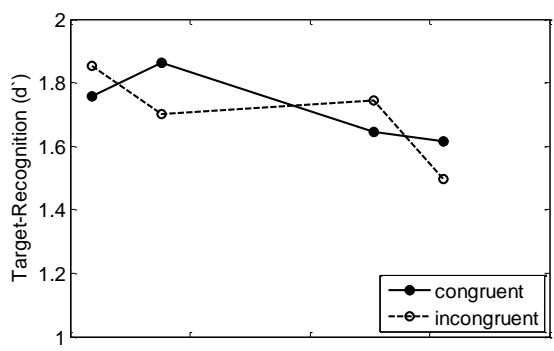

B

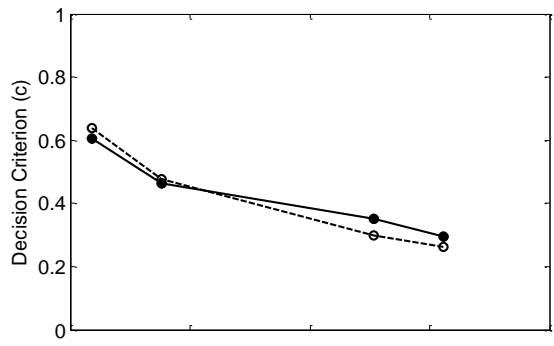

C

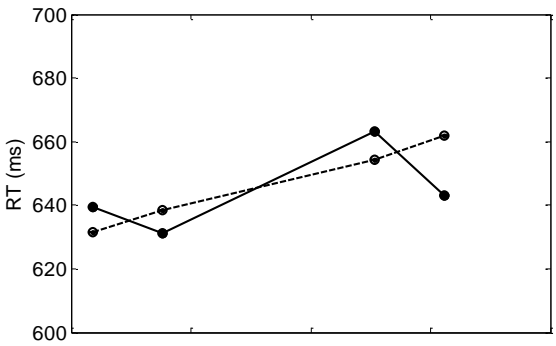

$\mathrm{D}$

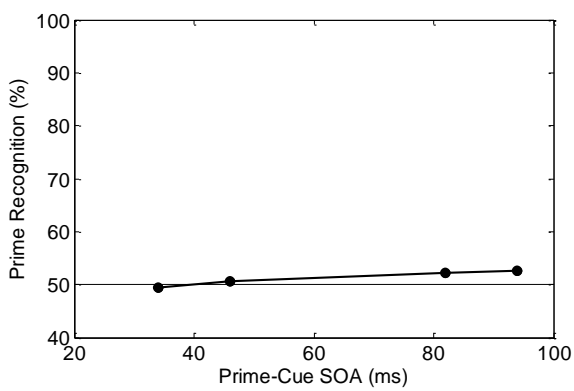

Figure 3.8 Results in Experiment 7. (A) $d^{\prime}$ as a measure of sensitivity for congruent and incongruent trials as a function of prime-cue-SOA. (B) $c$ as a measure of response criterion for congruent and incongruent trials as a function of prime-cue-SOA (C) Response times for congruent and incongruent trials as a function of prime-cueSOA. (D) Prime recognition performance in percent correct as a function of prime-cue-SOA.

comparing performance on congruent and incongruent trials showed no significant differences ( $p>.09$ in all cases). 
Criterion. Decision criterion values ( $c$, see Figure 3.8B) decreased with $\mathrm{SOA}, F(3,33)$ $=20, M S e=0.029, p<.001$, which indicates more conservative decision with short prime-cue SOAs than with long cue-target SOAs. The analysis of decision criteria revealed no effect of Congruency, $F(3,33)=0.6, M S e=0.004, p=.465$ nor an interaction of Congruency and SOA, $F(3,33)=0.9, M S e=0.011, p=.002$.

$R T$. RT (see Figure 3.8C) was neither significantly affected by Congruency, $F(1,11)=$ $0.3, M S e=385, p=.579$, nor SOA, $F(3,33)=2.1, M S e=1657, p=.116$, nor was there an interaction between the two factors, $F(3,33)=1.3, M S e=780, p=.28$.

Prime Recognition. Prime recognition performance (see Figure 3.8D) was unaffected by $\operatorname{SOA}, F(3,33)=1.3, M S e=0.076, p=.03$ and was not significantly better than chance, mean $d^{\prime}=0.076, t(11)=1.4, p=.179($ averaged across SOAs).

\subsubsection{Discussion}

Just like in Experiment 6 we failed to replicate any priming effects on target detection performance. This rules out that the absence of priming effects in Experiment 6 was due to the variation of cue-target SOAs in a wider range. Rather, it seems that the prime-cue configuration used in Experiments 6 and 7 is not suited to affect attention in a detection task.

\subsubsection{General discussion}

Two differences between the primes and cues used in Experiment 4 and those used in Experiment 6 and 7 might account for the discrepancy in priming effects. Firstly, prime recognition performance was better in Experiment 4 than in Experiments 6 and 7 where participants could not recognize primes with better than chance accuracy. To the extent that consciousness is critical for cue-priming effects in signal detection tasks, priming effects 
should covary with prime recognition performance. Accordingly, priming should be absent when primes are not visible, i.e. chance level performance in the recognition task.

Secondly, it might be that priming effects in Experiment 4 arise because primes affect cue processing but do not directly affect attention. According to this notion primes could facilitate or hinder cue processing at early levels of processing. One possible mechanism is priming based on perceptual similarity of prime and cue. Mattler (2006) addressed this problem in a different task by varying perceptual similarity. He found larger priming effects when primes and cues were perceptually similar than with dissimilar stimuli, showing that priming effects are in part based on perceptual similarity. If priming effects in Experiment 4 are entirely based on perceptual similarity the absence of priming in Experiments 6 and 7 could be explained be the relative absence of such similarity. Even though prime and cue symbols were the same in Experiments 6 and 7 as in Experiment 4 primes were never presented at the same location as the cue which might have prevented perceptual priming effects in Experiments 6 and 7. This issue was resolved by results of Experiment 5, where we manipulated similarity of prime and cue symbols and found that this was the critical difference between the former experiments.

\subsection{Summary of Project 2}

In Project 2, spatial cue-priming was transferred to a signal detection task. This task is more indicative of attentional processes at early levels of processing than the letter discrimination task in Project 1. Attentional effects in similar signal detection tasks have been taken as evidence for early selection. Primes and cues were similar to Project 1 with squares cueing the left side and diamonds cueing the right side of fixation. On each trial participants had to indicate whether a small white dot had been presented or not. The dot could be presented on top of a black square either to the left or to the right of fixation and its detection 
was made difficult by the presence of two random line masks which were presented immediately after target offset, one at each side. The relevant side on each trial was predicted with $100 \%$ validity by a cue symbol which was again preceded by a masked prime symbol. It has been argued that cueing effects on accuracy in detection tasks reflect early selection, because they cannot easily be explained at later decisional stages of processing. We used rather long cue-target SOAs because results of preliminary experiments suggested that priming effects might be larger with long cue-target SOAs, a finding that is consistent with findings from Luck and colleagues (1996) who suggested that cueing effects based on allocation of attentional resources require longer cue-target SOAs than cueing effects based on other processes such as reduction of spatial uncertainty. In Experiment 4, we found priming effects on detection accuracy which increased with prime-cue SOA. Due to the fact that there was confoundation of prime-cue SOA and cue-target SOA (prime-target SOA was held constant at $400 \mathrm{~ms}$ ) priming effects also decreased with increasing cue-target SOA. Therefore, we cannot be certain whether the effect of SOA on priming effects was caused by varying prime-cue SOA, varying cue-target SOA or both. Nevertheless, variation of priming effects with SOA was similar as in Project 1. In Experiment 6 we attempted to disentangle the effects of prime-cue SOA and cue-target SOA while at the same time improving masking of primes. To this end we varied cue-target SOA while keeping prime-cue SOA constant. In addition we increased masking by using star shaped mask stimuli instead of square and diamond stimuli and by presenting the primes randomly at one of two possible locations. However, in Experiment 6 there were no priming effects on target recognition performance. As we made several changes from Experiment 4 to Experiment 6, there are several explanations for this absence. We speculated that, apart from the changes in prime and cue stimuli which had successfully reduced prime visibility, the introduction of long cue-target SOAs might have reduced participants' attention to cues. To test this hypothesis we 
conducted a third experiment. In Experiment 7 we used the same prime and cue stimuli as in Experiment 6 combined with the same SOAs as in Experiment 4. Here, again, priming effects were absent which suggests that some attribute of primes and cues is critical for cue-priming effects on signal detection. Compared to the simple square and diamond stimuli in Experiment 4, the more complex combined square/diamond-and-star stimuli in Experiments 6 and 7 led to decreased visibility of primes, a smaller amount of attention devoted to the critical prime's location and decreased perceptual similarity between symbols presented at the same location.

From previous cue-priming experiments (Mattler, 2006) as well as Project 1 it seems that perceptual similarity leads to larger priming effects. With this in mind, we conducted Experiment 5, applying the same logic as in Experiment 3 (Project 1) to spatial cue-priming signal detection. In Project 2 we used the same symbols as primes and cues in Experiment 4 whereas in Experiments 6 and 7 there were no trials on which prime and cue were the same symbol configuration as the location of the relevant symbol always differed. Thus, it might be that priming effects in signal detection tasks can be explained solely on basis of perceptual priming of cue processing. If this is the case, priming effects on signal detection should only be present when prime and cue are the same symbol but not when they are only associated to the same side and perceptually dissimilar. This pattern is exactly what we found in Experiment 5 which suggests that prime processing does not extend beyond perceptual stages in a signal detection task, when attention operates at early stages of target processing.

In summary, priming effects on signal detection can be found but seem to be entirely explainable as resulting from perceptual priming of cue processing. This shows that access for masked stimuli to attentional processes at early stages of processing is limited compared to the access to attentional processes relevant in the letter discrimination task in Project 1 . In 
addition to an increase in accuracy in signal detection tasks, evidence for early selection by spatial attention has been provided by experiments that studied physiological markers of attentional selection. This approach was applied to spatial cue-priming in Project 3. 


\section{Project 3 - Spatial cue-priming effects on physiological measures of target processsing}

The following section was submitted as 'Masked Stimuli Modulate Endogenous Spatial Attention at Early Levels of Target Processing' by Simon Palmer \& Uwe Mattler to 'Psychophysiology.

\subsection{Experiment 8 - Masked stimuli modulate endogenous spatial attention at early levels of target processing}

\subsubsection{Abstract}

Unconscious stimuli can modulate motor behavior as well as other mental operations. Current research examined to what extent cognitive control operations are also susceptible to unconscious stimuli. One instance of cognitive control is involved in voluntary shifts of spatial attention. Here we recorded event-related brain potentials (ERPs) to gain insight into the mechanisms of effects of centrally presented masked symbolic cues on shifts of spatial attention. We found that masked primes modulate target related potentials, specifically N1 and N2, independently of the primes' visibility. We conclude that centrally presented masked primes can affect the allocation of spatial attention to early levels of target processing. These findings provide new evidence for unconscious effects on cognitive control operations. 


\subsubsection{Introduction}

Recent psychophysiological research has led to accumulating evidence that executive control processes, which were previously associated with conscious processing (e.g., Jack \& Shallice 2001; Dehaene \& Naccache, 2001) can be affected by unconscious stimuli (Krüger, Klapötke, Bode \& Mattler, 2013; Lau \& Passingham, 2007; Van Gaal, de Lange \& Cohen, 2012; Van Gaal \& Lamme, 2012). One instance of an executive control process, which provides an opportunity to study effects of unconscious stimuli on executive control processes, is the controlled allocation of visual attention in space. The literature on spatial attention distinguishes between processes of controlled allocation of attention (referring to endogenous or voluntary attention) and processes which affect spatial attention automatically (referring to exogenous attention; e.g., Jonides, 1981; Prinzmetal, McCool \& Park, 2005). Mechanisms of controlled shifts of spatial attention have been studied in spatial cueing tasks with centrally presented symbolic cue stimuli.

In a previous study, we reported effects of centrally presented masked symbolic primes on spatial attention in a spatial cue-priming task (Project 1). In this study, participants have been instructed to shift their attention to either the left or the right side of a screen according to the shape of a visible cue which has been presented centrally on the screen and predicted the position of a subsequently presented visual target with $100 \%$ validity. Before each cue, a masked prime has been presented, that was either similar to the following cue (congruent trials) or similar to the alternative cue (incongruent trials). The effect of these primes on target processing was reflected in faster and more accurate responses on congruent than on incongruent trials. This behavioral cue-priming effect suggests that masked primes affect the allocation of spatial attention. However, the mechanisms behind this effect are not entirely clear. The latter findings resulted from a speeded choice reaction time task in which 
each target stimulus was accompanied by an incompatible distractor stimulus. In another experiment, cue-priming effects have also been found with perceptually dissimilar prime-cue stimuli indicating that masked primes modulated relatively late levels of processes that are involved in shifts of spatial attention. In signal detection study, we found spatial cue-priming effects on detection performance of masked luminance targets (Project 2). This finding suggests that masked primes can affect shifts of spatial attention in such a way that early levels of target processing are modulated. However, the latter priming effects on early target processing depended on perceptual similarity between prime and cue stimuli. Therefore, we concluded that priming effects on detection performance likely result from an indirect effect of primes on the perceptual processing of the cues. However, all of these behavioral findings provide somewhat indirect evidence for the source and scope of the priming effects of masked stimuli. Therefore, in the present study we used electrophysiological measures to gain further insights into the mechanisms that are susceptible to masked stimuli in a spatial cue-priming task.

Several studies have combined spatial cueing with electrophysiological methods (e.g., Eimer, 1994; Mangun \& Hillyard, 1991; Luck et al., 1994) and found that spatial attention modulates early visual potentials that are evoked by a stimulus presentation. More specifically, P1 and N1 amplitudes elicited by laterally presented attended visual stimuli are typically enhanced compared to unattended visual stimuli over lateral occipital areas. P1 is a positive deflection in event-related potentials, usually peaking over occipital brain areas contralateral to the target location between 80 and $100 \mathrm{~ms}$ after target onset. These contralateral effects are followed by a similar effect $20 \mathrm{~ms}$ later over ipsilateral sites. $\mathrm{N} 1$ is a directly following negative component that occurs at similar sites with a peak latency of 150$200 \mathrm{~ms}$. P1 and N1 components have been associated with an activation of extrastriate brain 
areas of the visual cortex, specifically the posterior fusiform gyrus (Heinze et al., 1994). Mangun and Hillyard (1991) recorded brain potentials that were evoked by bright bar target stimuli presented against a dark background on either the left or right side of the screen. 800 ms before target onset, central arrow cues indicated the likely position of the target with a validity of $75 \%$. When participants were to discriminate whether the target bar was long or short, enhanced brain responses to the target stimuli in terms of the P1 and N1 component have been recorded. In contrast, in a simple target detection task enhancement was only found in the P1 component. P1 and N1 modulation by attention are associated with different effects of attention. These different effects can be dissociated in spatial cueing paradigms with neutral cues (Luck et al., 1994). The enhancement of the P1 component has been associated with attentional costs and the inhibition of ignored stimuli (Luck et al., 1994). In contrast, an enhancement of the N1 component has been associated with the benefits that are related to an enhanced processing of attended target stimuli (Hillyard \& Anllo-Vento, 1998). A similar pattern of results has been found in a visual search task (Luck \& Hillyard, 1995). These cueing effects on target processing have been considered early in terms of both time and anatomy in the visual hierarchy consistent with the view that spatial attention indeed modulates early processing of target stimuli. Applied to the spatial cue-priming paradigm, priming effects on early visual potentials would provide similar evidence for the view that masked primes can affect attention in such a way that early levels of target processing are modulated. Here we used a spatial cue-priming task similar to that of Mangun \& Hillyard (1991) and measured event-related brain potentials in response to target processing with a focus on early lateral occipital potentials P1 and N1. 


\subsubsection{Materials and Methods}

Participants. 22 Students (11 women, mean age: 22.7) participated in 3 sessions of the experiment in exchange for course credit or $35 €$. Three additional participants were excluded from analyses because of technical problems during EEG-recording. Another 5 participants were excluded during or after the practice session because of problems with eye-gaze detection. One additional participant showed systematic horizontal eye movements towards the target upon visual inspection of EOG-data and was excluded as well.

Task. Participants had to discriminate between long and short target bars in a speeded choice reaction time task. Responses were measured by button presses with the index fingers

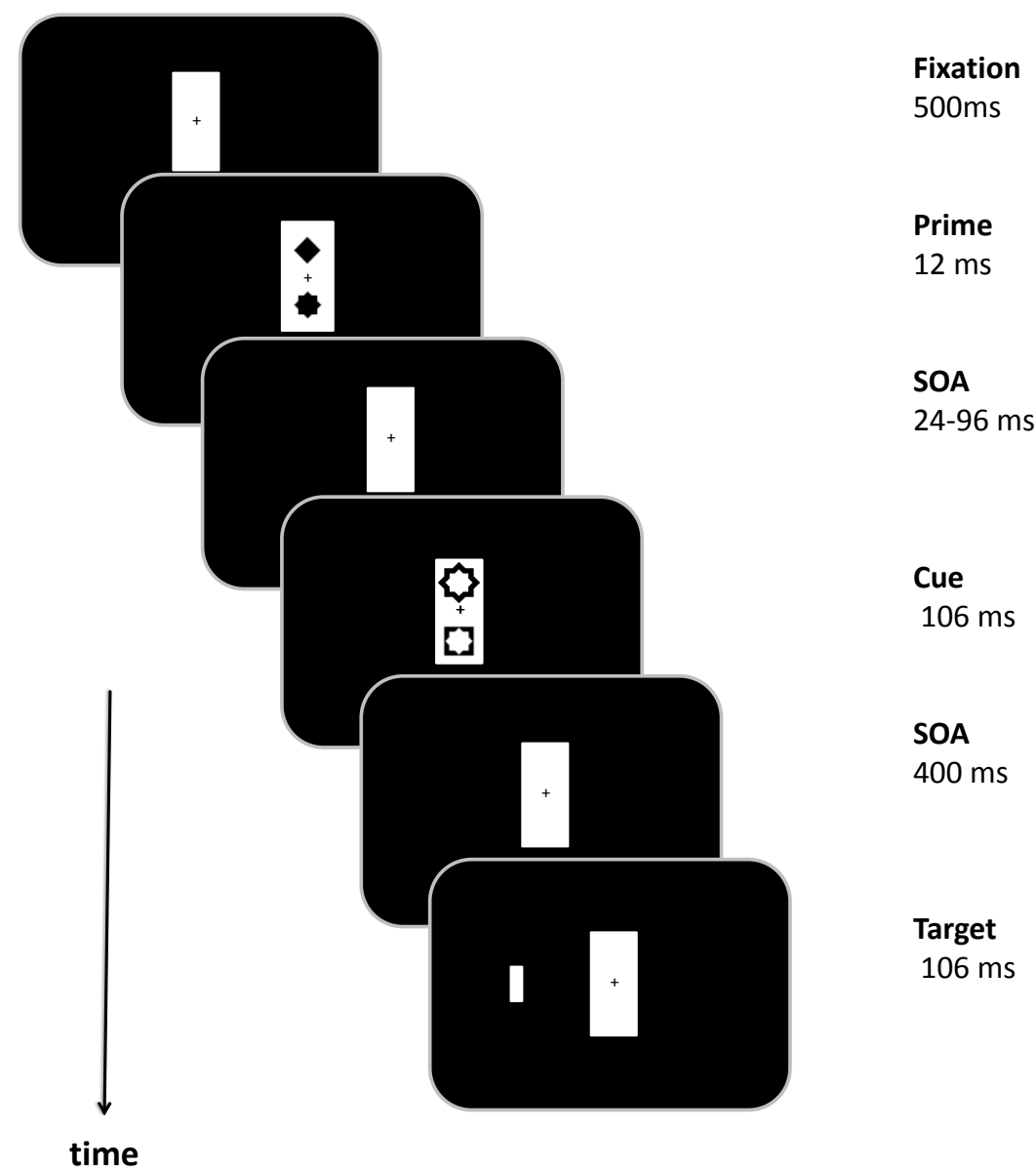

Figure 4.1 Sequence of stimulus events in a trial. 
of both hands on a keyboard in the practice session, and on a custom made response button box in the main session and in the prime recognition session. Target location was indicated by the shape of the cue with $100 \%$ validity. Square cues indicated a target on the left side and diamond cues indicated a target on the right side. Before the presentation of each cue a prime stimulus was presented which was either a smaller square or smaller diamond. Participants were not informed about the presence of prime stimuli until after the main session. In contrast to previous spatial cue-priming experiments (Project 1) no distractor stimulus was presented at the non-cued side. In a final prime recognition session, participants were instructed to indicate the identity of the prime stimuli (square or diamond).

Apparatus and Stimuli. Prime and cue stimuli had edge lengths of $1.07^{\circ}$ and $1.69^{\circ}$ visual angle, respectively. They were presented on top of a grey box in the center of the screen $4.55^{\circ} \times 7.79^{\circ}$ while the background of the screen was black (see Figure 4.1). The prime display consisted of the prime stimulus that was presented $1.4^{\circ}$ above or below fixation on $50 \%$ of the trials, respectively, and a star-shaped distractor stimulus at the opposite position. The cue display consisted of the cue stimulus that was presented $1.4^{\circ}$ below or above fixation on $50 \%$ of the trials, respectively, and a star-shaped distractor stimulus at the opposite position. On each trial, prime and cue stimuli were always presented at opposite locations. Therefore, at the position of the prime stimulus a star-shaped mask followed on each trial, and at the position of the star-shaped distractor in the prime display the visible cue followed. This was done because the star shaped stimulus was found to produce more effective masking than square and diamond masks. Targets were white bars that could be either long $\left(1.89^{\circ}\right)$ or short $\left(1.63^{\circ}\right)$ and were presented $5.5^{\circ}$ either to the left or right side of fixation. Primes were presented for $12 \mathrm{~ms}$, cues and targets were presented for $106 \mathrm{~ms}$ each. Prime-cue SOA varied randomly between trials in four steps ( $24 \mathrm{~ms}, 47 \mathrm{~ms}, 71 \mathrm{~ms}$, and $94 \mathrm{~ms}$ ) and cue-target SOA 
was hold constant at $400 \mathrm{~ms}$. Inter-trial-interval (from target onset to onset of the next prime) was fixed at $2012 \mathrm{~ms}$ and participants had to give their response in that time window.

The practice session took place in a special laboratory which was equipped to measure eye movements. We used an Eyelink 1000 infrared eye tracker to give participants online feedback about their eye movements and their blinking behavior. This was done verbally by the experimenter who could observe participants' eye movements online on the screen of the recording computer. Stimuli were presented on a CRT monitor (Viewsonic Perfect Flat 19"), which was placed in a viewing distance of $67 \mathrm{~cm}$ and participants performed the choicereaction time task.

In the main session, EEG was recorded with a BioSemi ActiveTwo recording system with 64 electrodes using the standard BioSemi head caps with electrodes arranged according to the International 10-20 system. Additionally, horizontal EOG was recorded as the difference between two electrodes at both external canthi, and vertical EOG was recorded as the difference between an electrode below the left eye and FP1. Two mastoid electrodes were used as reference. EEG-data were recorded with participants seated in a comfortable chair. As in the practice session, stimuli were presented on a CRT monitor (Viewsonic Perfect Flat 19"), which was placed outside the recording room and viewed through a window with a viewing distance of $133 \mathrm{~cm}$. The same setup was used in the prime recognition session but no EEG was recorded.

Design and procedure. The experiment consisted of one practice session of 45-60 minutes to familiarize participants with the task and train them to hold fixation. In the practice session, task difficulty was gradually increased by reducing both, the target length and the SOA. In this way, the conditions of the EEG-session were realized at the end of the practice session. EEG was recorded in one main session with 1920 trials in total. Finally, in a third 
session, prime visibility was measured under the same stimulation conditions as in the main session but without recording of the EEG and with only 640 trials. Each session comprised blocks of 64 trials that were separated by short breaks. In each block, each combination of 2 primes, 2 cues, 2 cue positions, 2 targets, and 4 SOAs was presented once. Thus, in the main session we collected a total of 240 congruent and 240 incongruent trials at each of the 4 levels of SOA. Both the main session and the prime recognition session began with a training phase with a few example trials and one block of 64 trials that was not included in the analyses.

Analysis of behavioral data. Analyses of behavioral data included only trials which were not excluded due to electrophysiological artifacts that were determined according to the criteria described below. Trimmed RT averages for correct responses were computed by excluding the three fastest and five slowest responses in each condition. Error rates were computed and arcsine transformed. These data were then analyzed using $2 \times 4$ repeatedmeasures-ANOVAs with factors Congruency and SOA.

ERP-Averaging and Analyses. Vertical and Horizontal EOG data were filtered with high pass and low pass filters set at 0.01 and $100 \mathrm{~Hz}$, respectively, and analyzed to exclude trials in which eye blinks or eye movements occurred. This was done by comparing the peak to peak difference on each trial (100 ms before prime onset until $800 \mathrm{~ms}$ after prime onset) to individually chosen thresholds. Thresholds were defined individually via visual inspection to include a minimum of trials with eye movements in the analyses and exclude at the same time a minimum of trials without eye movements. EEG was referenced to the average of both mastoid electrodes. Prime locked ERPs were computed for each condition with thresholds for trial exclusion set at $120 \mu \mathrm{V}$ for all EEG-channels relative to a baseline computed over 100 ms before prime onset and filtered using a low pass filter of $40 \mathrm{~Hz}$ and high pass filter of 1 Hz. In the end, each participant had at least 37 trials in each condition of the analyses. 
To determine priming effects on early visual evoked potentials of the target stimuli we examined target-locked ERPs at PO7/PO8 and compared congruent and incongruent trials with five different parameters that were determined in terms of the mean amplitude over specific time windows which were defined by visual inspection of grand average waveforms in all analyses. These time windows were centered approximately at the peak of the selected ERP-components. First, following Mangun and Hillyard (1991), we used a single time window to average evoked potentials for contralateral and ipsilateral target presentations. Visual inspection of the grand average waveforms shown in Figure 4.2 suggested to average contra- and ipsilateral P1 waveforms between $80 \mathrm{~ms}$ and $140 \mathrm{~ms}$ after target onset to determine Congruency effects on the P1 component. To determine Congruency effects on the $\mathrm{N} 1$ component, we averaged the contra- and ipsilateral N1 waveforms between $140 \mathrm{~ms}$ and $200 \mathrm{~ms}$ after target onset.

Second, to separate priming effects on P1 and N1 evoked by the target stimulus from any contamination of remaining effects of the preceding prime and cue stimuli on EEG waveforms, we computed the difference between mean P1-amplitude and mean N1 amplitude as a combined measure of attentional effects on target processing. This analysis eliminates additive effects of prime and cue processing which shift electrophysiological waveforms including P1 and N1 in one way on congruent trials and in another way on incongruent trials. Congruency effects on target processing that consist of attentional shifts of spatial attention at early levels of target processing should be reflected in the resulting P1-N1 amplitude difference.

Third, Mangun and Hillyard (1991) reported more pronounced effects of attention on early visual potentials at contralateral sites than at ipsilateral sites. To examine this aspect in our data, we conducted separate analyses of the contralateral components by averaging 
waveforms in the time windows from $80 \mathrm{~ms}$ to $120 \mathrm{~ms}$, and from $140 \mathrm{~ms}$ to $180 \mathrm{~ms}$, for the P1 and N1 component, respectively. These shorter time windows were chosen after visual inspection of grand average waveforms from contralateral electrodes because occipital P1 and N1 components usually occur with shorter latencies at contralateral sites than at ipsilateral sites.

Fourth, visual inspection of grand average waveforms in Figure 4.2 suggests priming effects on a later $\mathrm{N} 2$ component. To examine this effect, we determined the mean N2 amplitudes in the time window from $240 \mathrm{~ms}$ to $260 \mathrm{~ms}$ after target onset.

The effects of our independent variables on each of these electrophysiological components were analyzed in three steps. First, we conducted an omnibus analysis consisting of a $2 \times 2 \times 2 \times 4$ repeated-measures-ANOVA with factors Electrode Side, Target Side, Congruency, and SOA. Second, since we expected the largest priming-effect with the longest SOA at contralateral sites, we conducted a repeated-measures-ANOVA with factor Congruency only in the $94 \mathrm{~ms}$ SOA condition.

Finally, Mangun and Hillyard (1991) reported validity effects on the central N1 amplitude. Visual inspection of grand average waveforms in Figure 4.2 indeed suggests a priming effect on the central N1 component. Therefore, we determined the central N1 amplitude at $\mathrm{Cz}$ in the time window from $120 \mathrm{~ms}$ to $160 \mathrm{~ms}$. This component was analyzed by a 2 x 4 repeated-measures-ANOVA with factors Congruency and SOA which was followed by a repeated-measures-ANOVA with factor Congruency in the $94 \mathrm{~ms}$ SOA condition.

Analysis of prime-recognition data. Prime recognition performance was assessed by a signal-detection analysis resulting in measures of sensitivity in terms of d'. We computed d' separately for each participant with each SOA and each of the four different masks. Then we 
averaged d' across masks and analyzed recognition performance with a repeated-measuresANOVA with the factor SOA (Vorberg, Mattler, Heineke, Schmidt \& Schwarzbach, 2004). In addition, we performed t-tests for each SOA to test whether d' differed significantly from zero.

To assess the effect of prime visibility on the priming effects on different dependent variables, we separated participants in two groups with a median split of participants total prime recognition performance. In this way we introduced the between subjects factor Recognition Performance (poor vs. good). In addition, we conducted separate ANOVAs with the data of the $94 \mathrm{~ms}$ SOA condition because the largest priming effects occurred in this SOA condition. Across all analyses, reported p-values were Greenhouse-Geisser corrected where

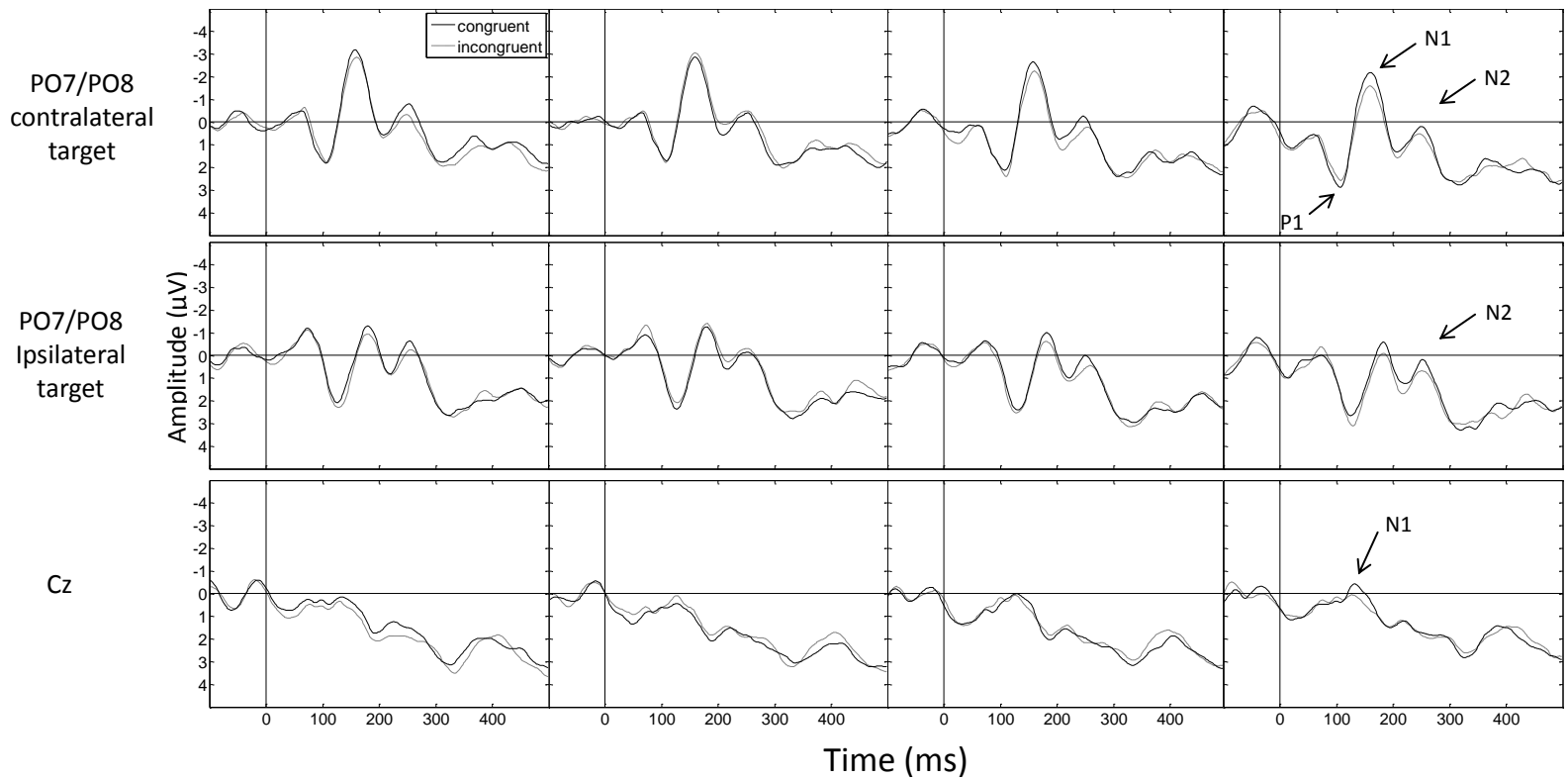

Figure 4.2 Target-locked grand averages. Top row: ERPs averaged over PO7 and PO8 for contralateral targets for congruent and incongruent trials with each SOA. Middle row: ERPs averaged over PO7 and PO8 for ipsilateral targets for congruent and incongruent trials with each SOA. Bottom row: ERPs averaged at $\mathrm{Cz}$ for congruent and incongruent trials with each SOA. 

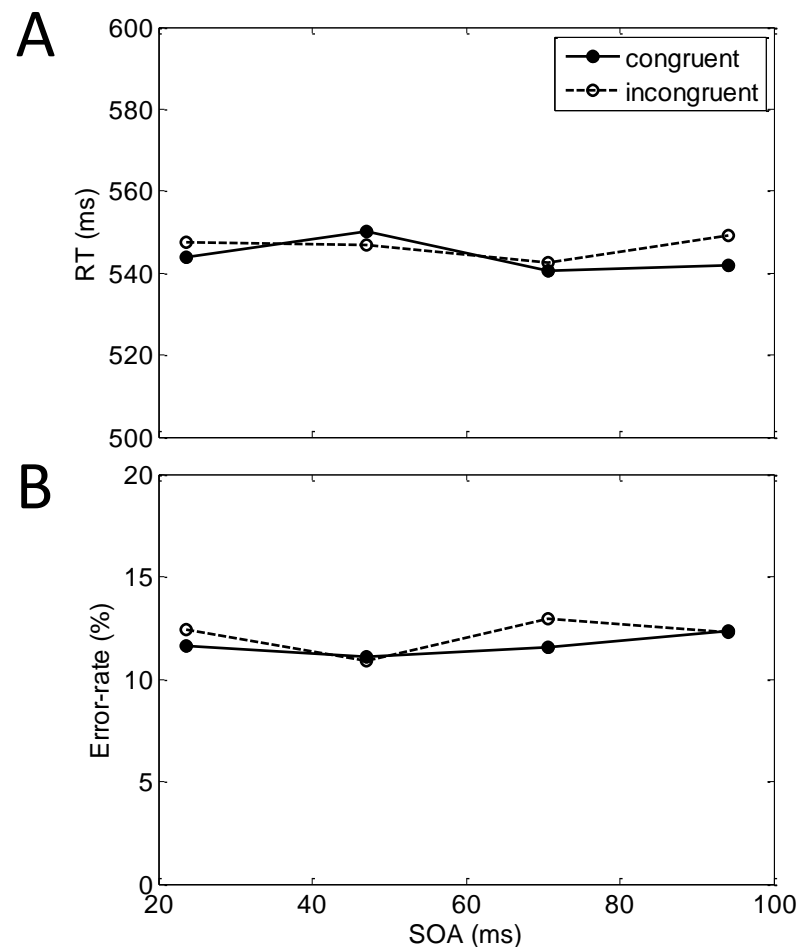

Figure 4.3 Behavioral Results. (A) Response time for congruent and incongruent trials as a function of primecue-SOA. (B) Error rate for congruent and incongruent trials as a function of prime-cue-SOA.

appropriate and uncorrected degrees of freedom are reported.

\subsubsection{Results}

Behavioral data

$R T$. Average RT is given in Figure 4.3A. Responses were not significantly faster on congruent (544 ms) than on incongruent trials $(546 \mathrm{~ms}), F(1,21)=1.8, p=.192, M S e=128$. There was a significant main effect of SOA on RT, $F(3,63)=3.0, p=.047, M S e=127, \varepsilon=$ 0.77. The interaction was not significant, $F(3,63)=1.8, p=.180, M S e=118, \varepsilon=0.47$. Planned t-tests for priming effects at each SOA, however, revealed that responses were $7 \mathrm{~ms}$ faster on congruent trials (542 ms) than on incongruent trials $(549 \mathrm{~ms})$ with $94 \mathrm{~ms}$ SOA, $t(21)$ $=2.8, p=.010$. 
Error rates. Figure 4.3B shows mean error rates across participants. Analysis of error rates revealed no significant effects $(F<2.1, p>.1$, in all cases).

\section{ERP results}

Target-locked grand averages are given in Figure 4.2. The values of the P1 and N1 component amplitudes determined in the corresponding time windows are given in Figure 4.4 as a function of Congruency and SOA averaged across electrodes (PO7 and PO8) and target side.

Occipital P1. P1 amplitude increased with SOA, $F(3,63)=5.8, p=.011, M S e=4.5, \varepsilon$ $=0.16$ and there was a significant Electrode Side $\mathrm{x}$ Target Side $\mathrm{x}$ SOA interaction, $F(3,63)=$ $3.5, p=.026, M S e=0.3, \varepsilon=0.77$ (Figure 4.4A). No other main effect or interaction reached significance $(F<1.6, p>.2$, in all cases $)$.

The effect of Congruency on the occipital P1 at contralateral electrodes in the $94 \mathrm{~ms}$ SOA condition did not reach significance, $F(1,21)=1.7, p=.202, M S e=1.1$ (Figure 4.5A).

Occipital N1. There was a marginal main effect of Congruency on N1 amplitude $F(1$, 21) $=3.9, p=.061, \mathrm{MSe}=1.4$, with more negative amplitudes on congruent $(-0.81 \mu \mathrm{V})$ than on incongruent trials $(-0.63 \mu \mathrm{V}$; see Figure 4.4B). The main effect of SOA was significant, $F(1,21)=7.3, p=.003, M S e=4.9$, which indicated that mean amplitude during the N1 period increased with SOA. In addition we found a significant Electrode Side x Target Side interaction, $F(1,21)=16.5, p=.001, M S e=25.3$, reflecting a difference in amplitude during the defined period between contralateral and ipsilateral electrodes. No other main effect or interaction was significant $(F<2.7, p>.11$, in all cases $)$. 


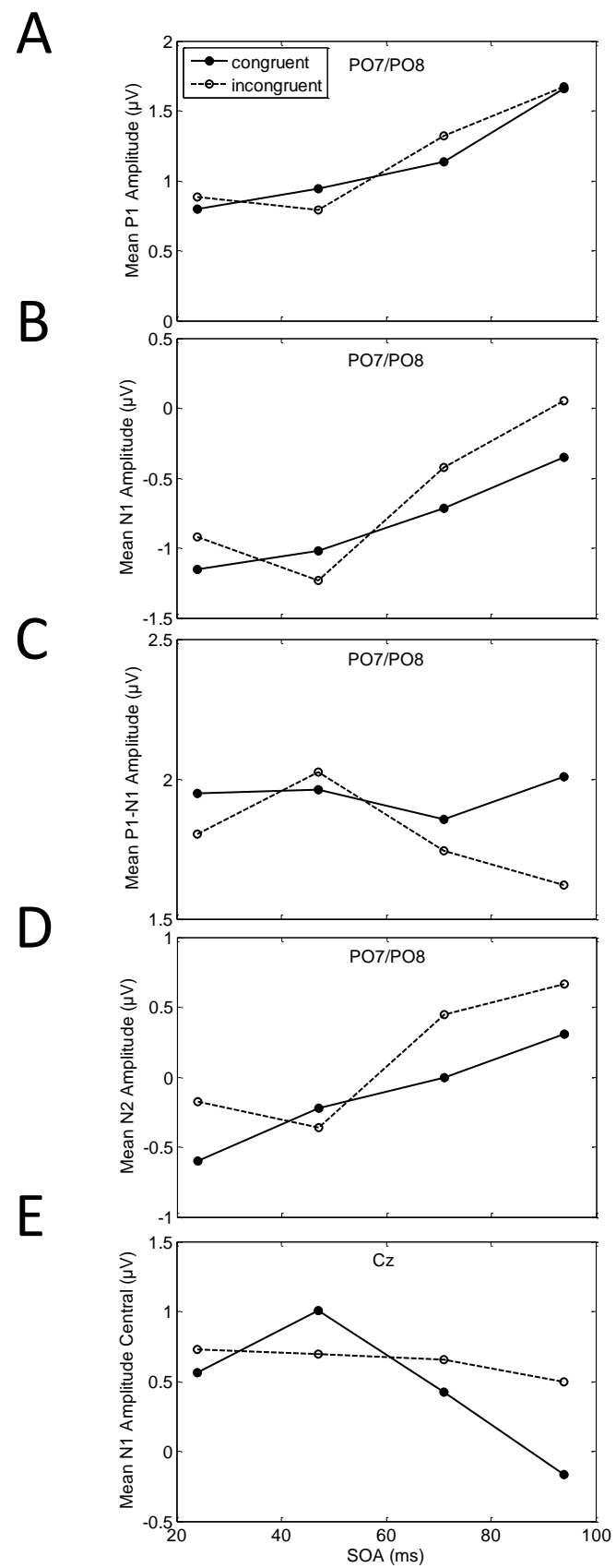

Figure 4.4 Mean amplitude of target-locked ERP components. (A) Mean P1 amplitude averaged at PO7 and PO8 for 80-140 ms after target onset for congruent and incongruent trials as a function of prime-cue-SOA. (B) Mean occipital N1 amplitude averaged at PO7 and PO8 for 140-200 ms after target onset for congruent and incongruent trials as a function of prime-cue-SOA. (C) Difference between occipital P1 amplitude and occipital N1 amplitude as a function of SOA. (D) Mean occipital N2 amplitude averaged at PO7 and PO8 for 240-260 ms after target onset for congruent and incongruent trials as a function of prime-cue-SOA. (E) Mean central N1 amplitude averaged at $\mathrm{Cz}$ for 120-160 ms after target onset for congruent and incongruent trials as a function of prime-cue-SOA. 
The N1 amplitude at contralateral electrodes with 94 ms SOA was significantly more pronounced on congruent $(-1.8 \mu \mathrm{V})$ than on incongruent trials $(-1.2 \mu \mathrm{V}), F(1,21)=5.5, p=$ $.029, M S e=0.6($ Figure 4.5B).

P1-N1 difference. We analyzed the amplitude difference between $\mathrm{P} 1$ and $\mathrm{N} 1$ as a measure of attention on early visual processing of the target stimulus that is less contaminated by the waveforms of the preceding prime and cue stimuli (Anllo-Vento, 1995). This difference was marginally affected by Congruency, $F(1,21)=2.9, p=.102, \mathrm{MSe}=1.3$ (Figure 4.4C) and there was a significant Electrode Side $\mathrm{x}$ Target Side interaction, $F(1,21)=$ 10.2, $p=.004, M S e=37.2$. No other effect reached significance $(F<1.7, p>.018$, in all

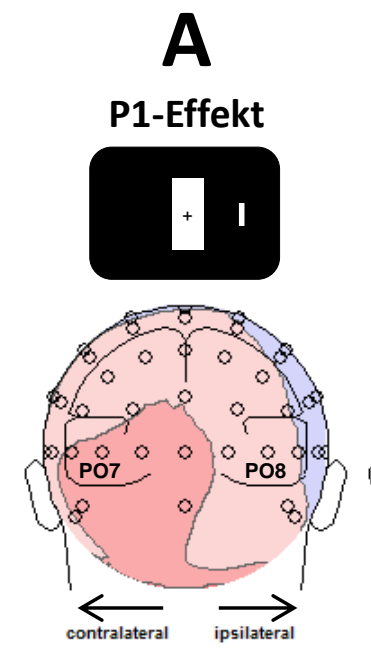

$80-140 \mathrm{~ms}$
B N1-Effekt
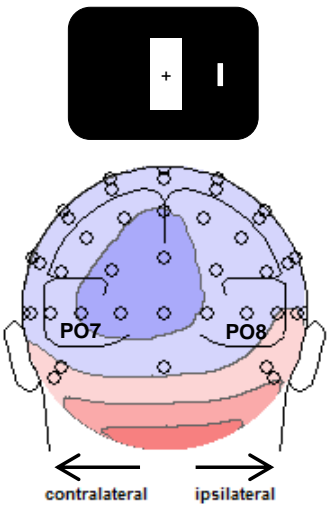

$140-200 \mathrm{~ms}$
C

P1-N1-Effekt
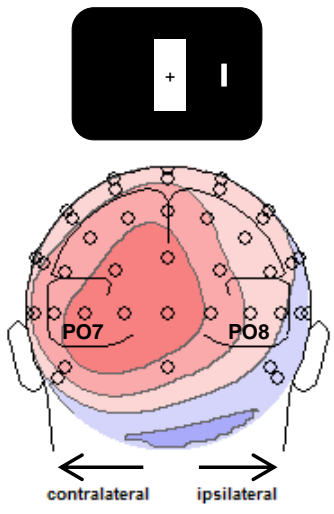

$80-140 \mathrm{~ms}-140-200 \mathrm{~ms}$
D N2-Effect
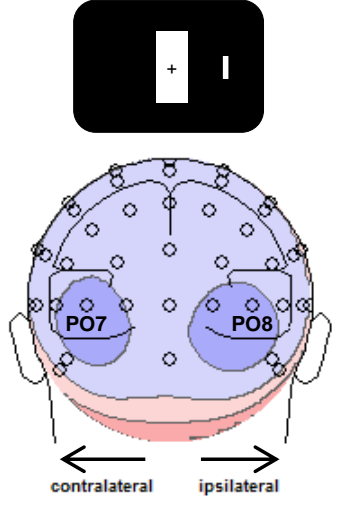

$240-260 \mathrm{~ms}$

Figure 4.5 Topographical distributions for priming effects with $94 \mathrm{~ms}$ on mean amplitudes for critical periods defined as mean amplitude on congruent trials - mean amplitude on incongruent trials. The ERP data were collapsed over cued side (left, right) and recording hemisphere (left, right) to show ipsilateral and contralateral ERP distributions on the right and left sides of the maps, respectively. Electrodes PO7 and PO8 which were used in the analyses are marked. (A) Priming effect in the P1 period (80 - $140 \mathrm{~ms}$ ). (B) Priming effect in the N1 period (140 - $200 \mathrm{~ms})$. (C) Priming effect on the P1-N1 difference. For this distribution we used the difference between mean P1 amplitude and mean N1 amplitude at each electrode to calculate priming effects. (D) Priming effect in the $\mathrm{N} 2$ period $(240-260 \mathrm{~ms})$. 
cases).

With $94 \mathrm{~ms}$ SOA the main effect of Congruency was significant at contralateral electrodes, $F(1,21)=9.3, p=.006, M S e=0.9($ see Figure $4.5 \mathrm{C})$.

Occipital N2. There was a main effect of Congruency on the occipital N2 amplitude, $F(1,21)=7.0, p=.015, M S e=1.9$. The amplitude of the occipital N2 was larger on congruent $(-0.13 \mu \mathrm{V})$ than on incongruent trials $(0.14 \mu \mathrm{V}$; see Figure 4.4D). This was qualified by a Congruency x Electrode Side interaction, $F(1,21)=5.4, p=.030, M S e=0.4$, indicating that priming effects were stronger at PO8 $(-0.38 \mu \mathrm{V})$ than at PO7 $(-0.16 \mu \mathrm{V})$. A main effect of SOA, $F(3,63)=5.7, p=.010, M S e=5.4, \varepsilon=0.25$, indicated that the amplitude of the N2 component decreased with increasing SOA. N2 was larger with left targets $(-0.41 \mu \mathrm{V})$ compared to right targets $(0.42 \mu \mathrm{V})$ which resulted in a main effect of Cued Side, $F(1,21)=$ $4.5, p=.045, M S e=26.8$.

Analysis of the Congruency effect on N2 amplitude at contralateral electrodes with 94 ms SOA revealed no significant main effect of Congruency, $F(1,21)=1.4, p=.245, M S e=$ 0.8 (Figure 4.5D).

Central N1. The analysis of the N1 component at $\mathrm{Cz}$ revealed a marginally significant Congruency x SOA interaction, $F(3,63)=2.4, p=.080, M S e=1.5, \varepsilon=0.92$ (see Figure 4.4E). No other effect reached significance $(F<2.5, p>.1$ in all cases). A separate ANOVA of the data with $94 \mathrm{~ms}$ SOA revealed a significant Congruency effect on central N1 amplitude: central N1 was larger on congruent $(-0.16 \mu \mathrm{V})$ than on incongruent trials $(0.49$ $\mu \mathrm{V}), F(1,21)=5.8, p=.025, M S e=1.7$. 
A

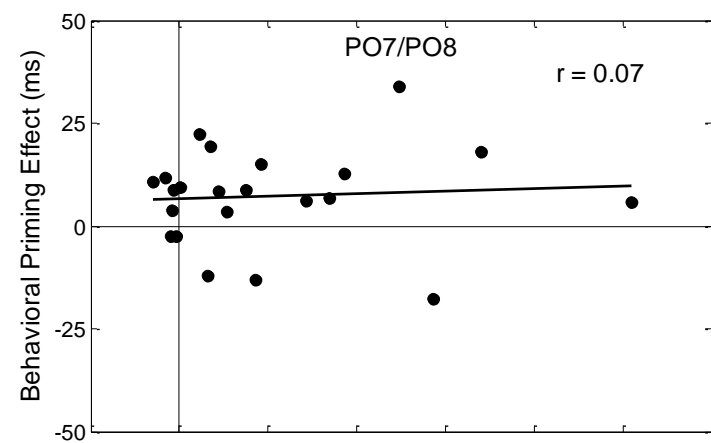

B

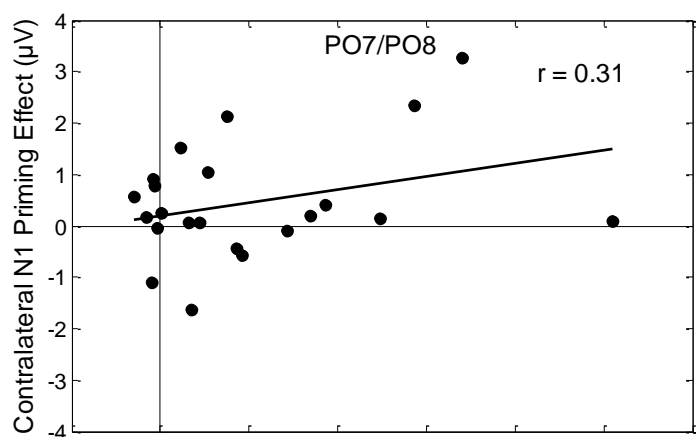

C

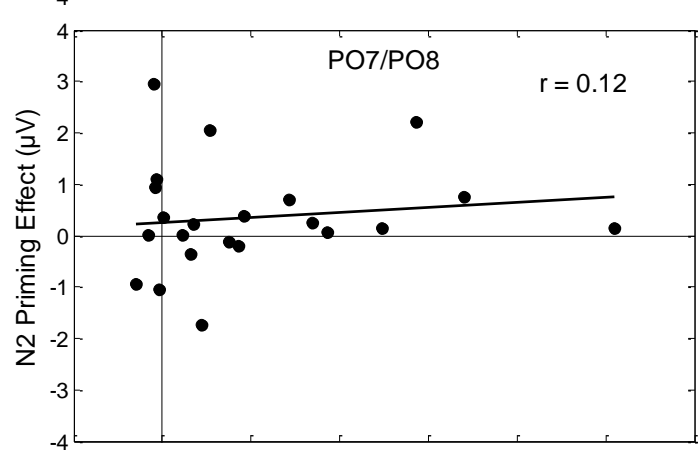

D

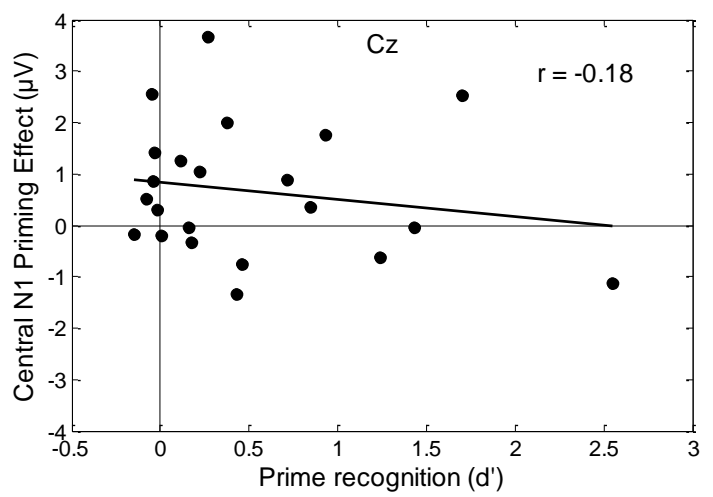

Figure 4.6 The relation between prime visibility and priming effects with $94 \mathrm{~ms}$ SOA. Scatterplots with regression lines of (A) priming effects on RT (defined as RT on incongruent trials minus RT on congruent trials), and average amplitudes (defined as average amplitude on incongruent trials minus average amplitude on congruent trials) of (B) the priming-effect on contralateral N1, (C) occipital N2, and (D) central N1 as a function of prime recognition performance. Pearson's $r$ is given in the top right corner. 
Prime recognition. The main effect of SOA on prime recognition performance was significant, $F(3,63)=7.3, p=.004, M S e=0.14 \varepsilon=0.24$, with d' values of $0.07,0.06,0.27$, and 0.51 with $24 \mathrm{~ms}, 47 \mathrm{~ms}, 71 \mathrm{~ms}$, and $94 \mathrm{~ms}$ SOA, respectively. Prime recognition performance was significantly better than chance with $71 \mathrm{~ms} \mathrm{SOA}, t(21)=3.3, p=.003$, and with $94 \mathrm{~ms} \mathrm{SOA}, t(21)=3.5, p=.002$. With $24 \mathrm{~ms}$ and $47 \mathrm{~ms}$ SOA, performance was not better than chance, $t(21)=0.9, p=.387$ and $t(21)=1.2, p=.257$, respectively.

In an attempt to clarify the role of prime visibility for the present priming effects, we separated participants in two groups according to their prime recognition performance averaged across all SOAs (median split). In mixed model ANOVAs with SOA and Congruency as within subject factor and Recognition Performance (good vs. poor) as a between subject factor we found no significance interaction which involved Recognition Performance and Congruency for either RT $(F<1, p>.4$, in all cases), contralateral occipital $\mathrm{N} 1(F<2.1, p>.1$, in all cases $)$, the occipital P1-N1 difference $(F<1.1, p>.3$, in all cases), nor bilateral N2 ( $F<1, p>.4$, in all cases $)$.

A similar pattern of results were revealed by paired t-tests to compare measures of priming effects in the condition with $94 \mathrm{~ms}$ SOA between participants with good and poor prime recognition performance (separated by a median split). Prime recognition performance in terms of d' did not differ from zero in the poor group (mean d' $=0.03, t(10)=0.8, p=$ .466) but was significantly above zero in the good group (mean d' $=1.0, t(10)=4.8, p<$ .001). Nonetheless, priming effects did not differ between groups (priming effect on RT with $7.3 \mathrm{~ms}$ vs. $7.1 \mathrm{~ms}$, for the good and poor group, respectively, $t(21)=0.04, p=.966$; priming effect on the P1-N1 difference with $0.27 \mu \mathrm{V}$ vs. $0.47 \mu \mathrm{V}$, for the good and poor group, respectively, $t(21)=0.4, p=.661$; on central $\mathrm{N} 1$ amplitude with $-0.66 \mu \mathrm{V}$ vs. $-0.66 \mu \mathrm{V}$, for the good and poor group, respectively, $t(21)=0.01, p=.989$; on occipital N2 amplitude $-0.58 \mu \mathrm{V}$ 
vs. $-0.13 \mu \mathrm{V}, t(21)=1.0, p=.340)$. Figure 4.6 shows the scatterplots for these comparisons on the basis of individual participants' priming effects and their recognition performance in the $\mathrm{SOA}=94 \mathrm{~ms}$ condition. There was not one significant correlation $(-.19<r<.32, p>.1$, in all cases). Consistently, these findings suggest that the priming effects were independent from the visibility of the prime.

\subsubsection{Discussion}

When participants had to report the length of a single visual target stimulus that appeared at a validly cued position we found a significant effect of prime-cue congruency on reaction time. This replicates a similar finding from an earlier choice reaction time study (Project 1) indicating that masked primes modulate the allocation of spatial attention. In contrast to the previous study, the behavioral priming effect of the present experiment was substantially smaller and occurred only with a long SOA. Beyond the behavioral effect, brain potentials that reflect target processing were also modulated by the prime-cue congruency. With 94 SOA, primes affected central as well as contralateral occipital N1 amplitude, and the lateral occipital N2 amplitude, but there was no significant priming effect on the occipital P1 amplitude. As mentioned above, a problem with the analysis of P1 and N1 components in the present experiment consists in a potential contamination of the target evoked components by propagated shifts of the waveforms that are caused by prime and cue processing. Substantial effects of prime-cue SOA were found in visual potentials even after target onset despite the long 400 ms cue-target SOA which are likely to reflect prime and cue processing. If prime and cue processing differs between congruent and incongruent trials these differences may be falsely attributed to effects on target processing. Note, however, that this problem constrains only definite conclusions regarding the issue whether P1 and N1 are specifically modulated and thus which specific mechanisms of target processing are affected by priming. We tried to 
circumvent this problem by using a relatively long cue-target SOA as well as using the P1-N1 difference as a measure of attentional effects. The modulation of the P1-N1 difference by masked primes can be interpreted as evidence for priming effects on early target processing.

\subsubsection{Mechanisms of Spatial Cue-priming}

There was a significant modulation of both target N1 and target N2 by masked primes' congruency to the following spatial cue. Moreover, these priming effects were not modulated by participants' ability to distinguish between the primes. This finding suggests that masked primes can influence the control of spatial attention with the effect that early visual target processing is modulated. In contrast to previous spatial cueing experiments that used visible cues to shift attention (e.g., Mangun \& Hillyard, 1991; Luck et al., 1994), our masked primes did not affect the P1 component of target processing significantly although the present task was similar to that of Experiment 1 of Mangun and Hillyard (1991). On the one hand, the absence of a significant effect in our study might result from a smaller effect of masked congruent and incongruent primes compared to the effect of the validity of the visible cues used by Mangun and Hillyard (1991). Another difference between our experiment and the experiment of these authors consists in the fact that we did not have any trial with invalid visible cues which were part of the previous study. The absence of the invalid cues might lead to differences in the mechanisms that contribute to priming-effects in the two tasks. It has been suggested that modulation of target N1 by attention reflects application of a limited capacity target discrimination mechanism (Luck, 1995; see also Vogel \& Luck, 2000), whereas P1 effects reflect noise suppression in the presence of confusable distractors. The enhancement of the P1 amplitude has been associated with attentional costs rather than benefits because P1 is not enhanced on validly cued trials compared to trials with neutral cues but it is reduced on invalid trials (Luck, 1995). Therefore, we speculate that priming effects 
on P1 amplitude were absent in the present experiment because incongruent primes were followed by visible cues that were always valid and did not produce sufficient costs in terms of shifting attention to the non-cued side. Thus, the finding that primes affected N1 amplitude but not P1 amplitude in the present experiment is in line with previous findings and supports the view that masked primes affect spatial attention similarly to visible cues.

In addition, we found a priming effect on the occipital N2 component. This N2 effect might be comparable to a similar $\mathrm{N} 2$ effects that has been reported in a spatial cueing experiment of Eimer (1993). A similar N2 effect can be found in the experiments of Mangun and Hillyard (1991, Figures 1 and 2) in a time period termed late positive deflection by these authors. According to our knowledge, the processes that are reflected by this N2 modulation are not clear.

\subsubsection{Comparison of the Present Priming Effects and Other Spatial Cue-Priming Effects}

Reliable behavioral priming effects were found only with the longest SOA in the present experiment. This finding contrasts with cue-priming effects that increased monotonically with increasing prime-cue SOA both with non-spatial (Mattler, 2003) and spatial cue-priming tasks (Project 1). At least three aspects of the present study might have contributed to this difference. First, previous studies have already shown that the magnitude of cue-priming effects is stimulus and / or task-dependent (Mattler, 2003; 2005). Along this line, the present finding demonstrates that cue-priming effects in the speeded bar discrimination task are smaller than the effects in the speeded letter discrimination task (Project 1). Second, the present experiment differs from previous cue-priming experiments because we used a long cue-target SOA of 400 ms as suggested by Luck, Hillyard, Mouloua, and Hawkins (1996). These authors proposed that early effects of attention manifest only with long cue-target SOAs because it takes time to allocate resources according to cues. For cue- 
priming, however, it has been shown before that priming effects decrease with increasing cuetarget SOA (Mattler, 2005; Project 1). This decay of priming effects might contribute to the small magnitude of the present priming effects on RT. Third, participants did not know in advance whether the cue appears above or below fixation in the present experiment. Therefore, they had to divide their attention across the two possible cue positions. This might have reduced the effect of the prime because it has been found that effects of masked primes are modulated by spatial attention (Marzouki, Grainger \& Theeuwes, 2006; Van den Bussche, Hughes, Van Humbeeck \& Reynvoet, 2010).

\subsubsection{Correlates of Shifts of Attention}

Several spatial cueing studies have reported electrophysiological evidence for an active shift of spatial attention in response to the spatial cue prior to target presentation (e.g., Harter, Miller, Price, LaLonde \& Keyes, 1989; Hopf \& Mangun, 2000). However, it has been disputed whether these findings reflect effects of cue processing instead of true markers of shifts of attention (Van Velzen \& Eimer, 2003). In these studies, the electrophysiological markers for shifts of spatial attention in response to the cue have been determined by the comparison between waveforms at sites contralateral to the cued location with those at ipsilateral sites. However, the cue-priming paradigm of the present study differs from traditional cueing paradigms because in our experiment a visible cue stimulus with $100 \%$ validity has been presented on each trial. Therefore, it is questionable to compare the effects of masked primes with previously published effects of visible cues. Instead, it would have been surprising if the masked primes used in our paradigm had produced comparable effects. However, our analyses of cue/prime locked waveforms in the temporal interval before target onset revealed no evidence for a lateralized shift of attention. Therefore, it remains as an interesting question for future research to examine whether subliminal endogenous stimuli 
have the power to generate EEG-correlates of shifted spatial attention. We are not very optimistic that the cue-priming paradigm could produce such an effect because in this paradigm the masked primes are always followed by a highly task relevant visible cue which is most likely dominating any prime induced shifts of attention.

\subsubsection{Role of Perceptual Similarity between Prime and Cue}

In two previous spatial cue-priming studies we found priming-effects that were at least partially based on perceptual priming of cue processing which might have propagated to a priming effect on target processing (Projects 1,2). To eliminate similar perceptual priming effects in the present experiment, we used a stimulus display in which prime and cue stimuli appeared at opposite positions (see Figure 4.1). This prime-cue stimulus display has been suggested by an unpublished experiment which used the same display and was run to repeat our previous cue-priming experiment with the signal detection task (Project 2). The cuepriming effects that we had found with the signal detection task when primes and cues were perceptually similar (Project 2) were absent when prime and cue stimuli appeared at opposite positions. Therefore, we believe that the present prime-cue stimulus display does not include perceptual priming of cue processing. This view is further supported by unreported analyses of prime-locked ERPs of the present experiment which show that early visual responses to the cue were not modulated by the congruency of the previously presented prime stimuli, although such an effect has been observed in an unpublished experiment where prime and cue stimuli occurred at the same position. In sum, we think the present priming effects on target processing do not result from perceptual prime-cue interactions. 


\subsubsection{Role of Prime Visibility in Priming of Spatial Attention}

Previous cue-priming studies have demonstrated the independence of priming effects and prime visibility under similar circumstances by showing increasing cue-priming effects with decreasing prime visibility (Mattler, 2003; Lau \& Passingham, 2007). This was replicated in the present study where we did not find any significant correlation between cuepriming effects and prime visibility. Priming effects were the same with participants who could recognize primes no better than chance and those who were clearly above chance in the prime recognition task. These findings from objective tests are supported by subjective reports of our participants because only 11 out of 22 reported that they had seen the primes. In sum, the data suggests that the present cue-priming effects do not depend on prime visibility.

\subsubsection{Conclusion}

The present experiment shows that masked primes can modulate early levels of target processing in a spatial cue-priming task. With long SOA priming effects occurred on behavioral measures and on target $\mathrm{N} 1$ amplitude independent from the visibility of the effective stimuli. These priming effects are unlikely due to perceptual priming of cue processing because prime and cue stimuli were perceptually dissimilar. Therefore, the present findings complete previous findings of cue-priming effects on shifts of spatial attention (Projects 1, 2). Findings suggest that unconscious stimuli can affect the allocation of spatial attention at early levels of target processing.

Following on the finding that masked primes can affect target-locked potentials, we conducted two further experiments with moderate success. Experiment 9 aimed at replicating priming physiological priming effects in a letter discrimination task and establishing whether primes would affect processing of stimuli presented at non-cued positions. Such modulation was not found, which might have been due to selection of too short cue-target SOAs for 
priming effects on early stimulus processing. Priming effects on target related ERPs could not be unequivocally attributed to target processing due to overlaps with cue related shifts of potential, which were also modulated by primes. Experiment 10 examined the role of cuetarget SOA and the presence of distractor stimuli at the non-cued position. Surprisingly, priming effects reversed with long cue-target SOA in Experiment 10 which makes interpretation of results difficult. These two experiments are reported in the following.

\subsection{Experiment 9 - Priming effects on stimuli presented at non-cued}

\section{locations}

Considering the small priming effects on both behavioral and electrophysiological measures in Experiment 8, we conducted another experiment with the letter discrimination task that had been found to produce larger priming effects in Project 1 . To this end, we used the same task as in Project 1. Participants were to identify letters at the cued side, which were accompanied by a distractor letter at the non-cued side. With these targets, we expected larger behavioral priming effects than in Experiment 8, possibly because they increase participants' motivation to use the cues. In addition, we added irrelevant unilateral probe stimuli with the idea that measuring ERPs elicited by these probes would allow us to disentangle attentional effects of primes and effects of primes on cue processing. ERPs to such irrelevant stimuli have been shown to be modulated by attention (Luck \& Hillyard, 1995). For the cued side, we expected potentials to be increased on congruent compared to incongruent trials, reflecting the same priming effects found in Experiment 8. If primes can cause shifts of attention, possibly more resources are devoted to processing input at the non-cued side on incongruent than on congruent trials. This may lead to increased early visual potentials (P1 and/or N1) to probe stimuli presented at the non-cued side on incongruent trials compared to congruent trials. Thus, enhanced processing of probes on incongruent trials at the non-cued location would 
indicate that primes can direct attention independently of the cue. Probe elicited ERPs were isolated by computing the difference in ERPs between trials on which a probe was presented and trials on which the probe was omitted. In addition, we hoped that this experiment could be informative about potential differences between the letter discrimination task and the bar task. We used a shorter cue-target SOA than in Experiment 8 because priming effects in the letter discrimination task were found to be maximal with short cue-target SOAs.

\subsubsection{Method}

Participants. 15 participants (10 women, mean age: 23$)$ took part in 4 sessions of the experiment. 2 additional participants were excluded from analyses due to too many eye movement artifacts. Another 2 participants were excluded during their practice sessions

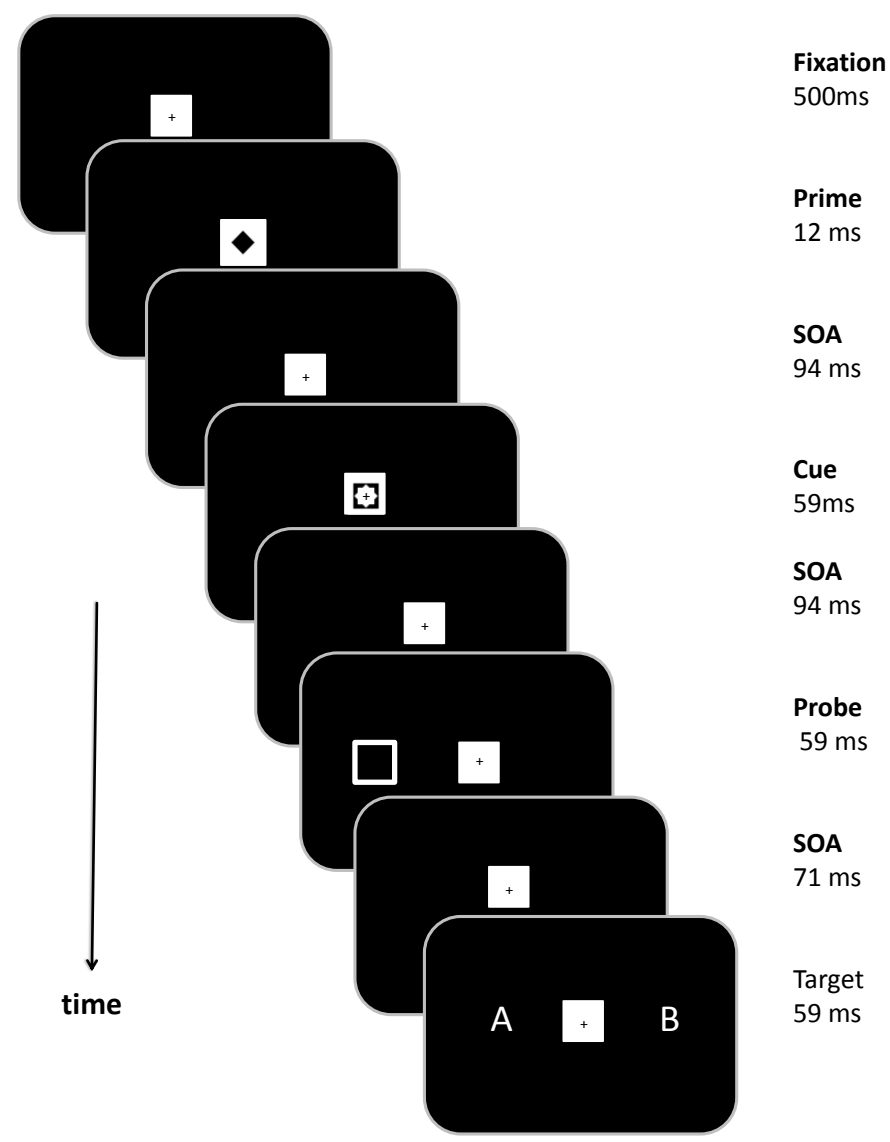

Figure 4.7 Sequence of stimulus events in a trial of Experiment 9. 
because of problems with eye gaze detection.

Task. We used essentially the same task as in Project 1. Participants had to indicate whether the target letter on the cued side was an A or a B by pressing buttons on a keyboard or a response box (EEG-session) as fast and as accurately as possible. They were informed that the probe stimuli were irrelevant to their task and were not predictive of target locations.

Apparatus and Stimuli. Stimuli were presented on Viewsonic 19" Perfect Flat monitors with a refresh rate of $85 \mathrm{~Hz}$. Participants were seated in a viewing distance of $67 \mathrm{~cm}$ in the practice session and $130 \mathrm{~cm}$ in the EEG-sessions and the prime recognition session. Targets were letters A and $\mathrm{B}$ (size $0.65^{\circ} \times 0.43^{\circ}$ ) presented $5.5^{\circ}$ to the left or right of fixation. Probe stimuli were square frames around target position (edge length $1.3^{\circ}$, line width: $0.09^{\circ}$ ). Figure 4.7 gives an example of the stimulation in Experiment 9.

Design and procedure. Participants completed one practice session and 2 EEG sessions, each with 1600 trials, and a prime-recognition session. The EEG sessions comprised 20 Blocks of 80 trials. In each block, each combination of 2 Primes, 2 Cues and 2 targets was presented twice with a probe at the cued side, twice with a probe at the non-cued side, and once without a probe. Thus, probes were presented on the cued or the non-cued side on two fifth of all trials respectively and were absent in the remaining fifth of trials (no probe trials).

ERP-Averaging and Analyses.

Target-locked ERPs and probe-locked ERPs were averaged using the same procedure as in Experiment 8. Probe induced ERPs were obtained by subtracting the average for no probe trials from the average for the respective probe trials (black in Figure 4.9) i.e. trials on which the same prime and cue were presented but a probe was presented.. From these probeno probe differences, we defined time windows for probe $\mathrm{P} 1$ and $\mathrm{N} 1$ by visual inspection. As 
P1 and N1 components were diminished at ipsilateral electrodes compared to no probe trials, (see Figures 4.9 and 4.11C) we used only contralateral electrodes in a second analysis to asses probe elicited P1 and N1 components. Periods used for P1 and N1 were shorter than those in Experiment 8 because they were chosen to fit only the contralateral P1 and N1 components which occur earlier than their ipsilateral counterparts. P1 amplitude was averaged at PO7/PO8 for contralateral probes from $80 \mathrm{~ms}$ to $120 \mathrm{~ms}$ and $\mathrm{N} 1$ amplitude from 120 to $160 \mathrm{~ms}$. Averages were then entered into $2 \times 2 \times 2 \times 2$ repeated measures ANOVAs with factors Electrode Side, Probe Side, Cued Side, and Prime-Cue Congruency.

In addition, target-locked ERPs from no probe trials were used to compute target related P1 (80-140 ms) and N1 (140-200 ms) components using mean amplitude at PO7/PO8. Visual inspection of target-locked ERPs suggested additional later effects of Congruency at frontal, parietal and occipital electrodes. A frontal negative component was averaged at FP1/FP2 from 130 to 170 ms. A later, widespread P3 component was analyzed in two ways once using averages at FC3/FC4 from 250-300 ms and once averaging at PO7/PO8 from 250300 ms. Target related components were analyzed with 2 × 2 × 2 repeated measures ANOVAs with factors Electrode Side, Cued Side, and Congruency. Additionally, RT and error rates were averaged like in Experiment 8 and analyzed with $3 \times 2$ repeated measures ANOVAs with factors Probe Location (cued, non-cued, no probe) and Prime-Cue Congruency. For brevity, only significant effects in the conducted ANOVAs are reported. All non-reported main effects and interactions were not significant. $d$ ' for prime recognition performance was computed by defining square primes as signal and diamond primes as noise. This measure of recognition performance was averaged across both masks and tested against 0 using a single sample t-test. 
A

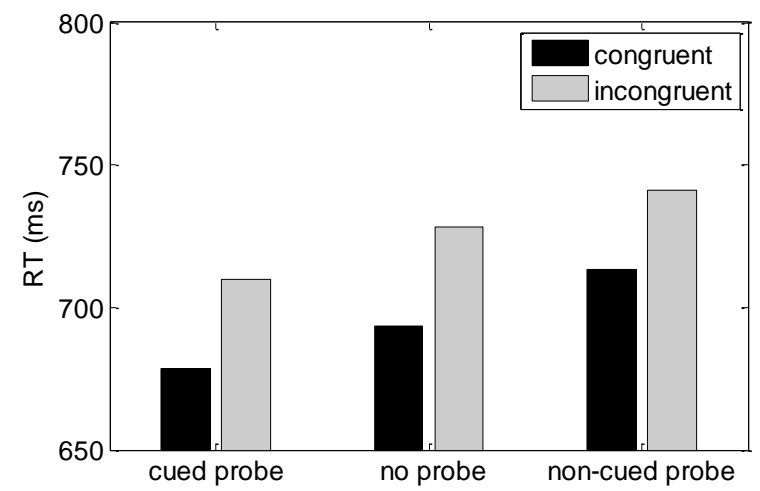

B

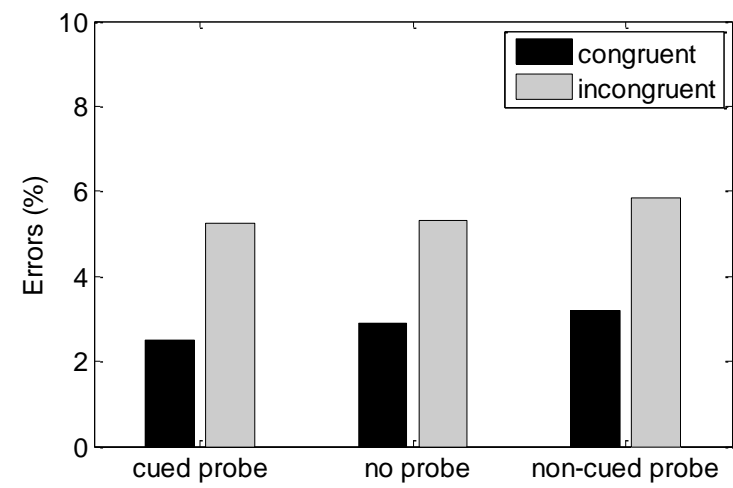

Figure 4.8 Results in Experiment 9 (A) RT on congruent and incongruent trials in Experiment 9 for the three different probe locations. (B) Error rates on congruent and incongruent trials in Experiment 9 for the three different probe locations.

\subsubsection{Results}

$R T$. Mean RT for the conditions in Experiment 2 is given in Figure 4.8A. There was a main effect of Probe Location, $F(2,28)=21.3, M S e=384, p<.001$. Responses on trials with no probe $(711 \mathrm{~ms})$ were slower than on trials on which the probe appeared at the cued side (694 ms), but faster than on trials on which the probe appeared at the non-cued side (727 ms). Post-hoc t-tests: $t(14)=3.4, p=.004$ for cued-probe vs. no probe, $t(14)=4.9, p<.001$ for non-cued probe vs. no probe. We also found a main effect of Congruency, $F(1,14)=13.2$, $M S e=1670, p=.003$, indicating faster responses on congruent $(695 \mathrm{~ms})$ than on incongruent trials $(726 \mathrm{~ms})$. The interaction between Congruency and Probe Location was not significant $(F<2, p>.2)$

Error rates. Probe Location had an effect on response errors rates, $F(2,28)=3.4, M S e$ $=0.003, p=.05$ (Figure 4.8B). Numerically, errors were committed on $3.9 \%$ of trials with cued probes, $4.5 \%$ of trials with non-cued probes and $4.1 \%$ of trials without probe. However, 
post hoc t-tests did not reveal significant differences between the probe locations, $t\langle 2, p\rangle$ .07 , in all cases.

Prime Recognition. Prime recognition performance was significantly better than chance $($ mean d' $=1.7, t(14)=5.5, p<.001)$.

\section{Probe induced ERPs}

P1. Figure 4.9 shows the ERPs used to isolate probe induced shifts of potential. Voltage maps for this period are shown in Figure 4.11A. We averaged P1 for the probe - no probe difference from $80 \mathrm{~ms}$ to $120 \mathrm{~ms}$ after probe onset. A significant Electrode Side $\mathrm{x}$ Probe Side interaction, $F(1,14)=20.9, p<.001, M S e=3.0$, indicated that this difference was positive at the contralateral electrode $(0.75 \mu \mathrm{V})$ whereas the opposite was true for the ipsilateral electrode $(-0.28 \mu \mathrm{V})$. There was an Electrode Side $\mathrm{x}$ Cued Side interaction indicating that P1 was enlarged when the left side was cued at PO8 (left-right difference: 0.37

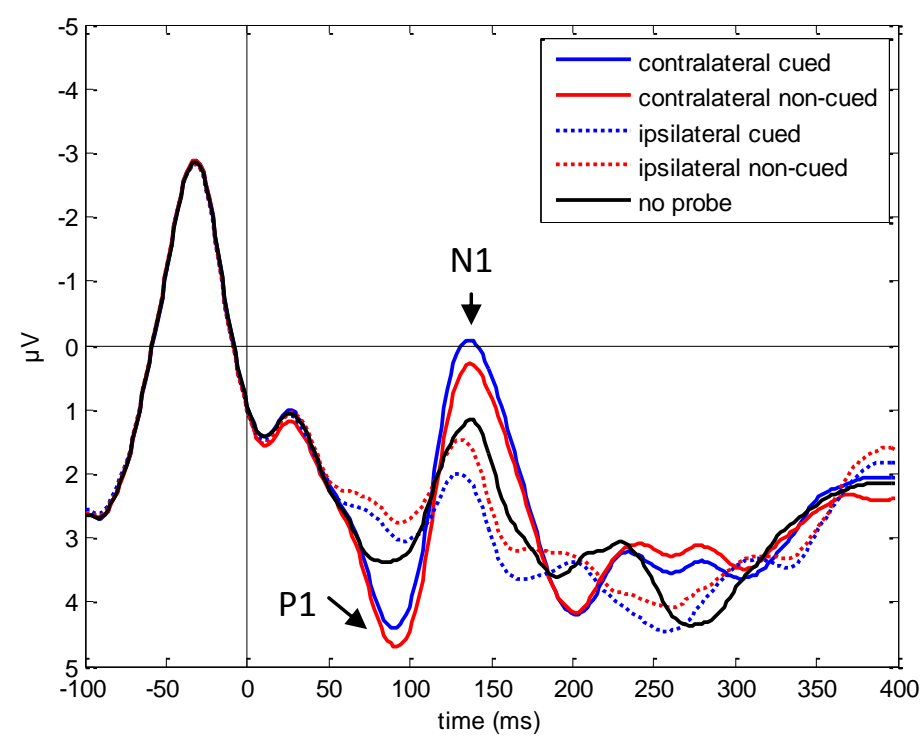

Figure 4.9 Probe locked potentials at PO7/PO8. Probe ERPs were collapsed over left- and right-cue conditions and left and right hemispheres to obtain waveforms recorded and contralaterally (left panels) and ipsilaterally (right panels) to the side of the cue. No probe potentials were averaged over PO7 and PO8 for all no probe trials. 
$\mu \mathrm{V})$ but not at PO7 $(-0.05 \mu \mathrm{V}), F(1,14)=7.9, p=.014, M S e=0.3$. Note, that this indicates an interaction of Cued Side and probe presence rather than a cueing effect because of the difference waveform. P1 was increased on congruent compared to incongruent trials for right probes (priming effect: $0.22 \mu \mathrm{V}$ ) but not for left probes (priming effect: $0.09 \mu \mathrm{V}$ ), as indicated by a Probe Side x Congruency interaction, $F(1,14)=5.7, p=.031, M S e=0.26$.

N1. For the period defined for the probe N1 (120-160 ms, Figure 4.11B), we found a main effect of Congruency, $F(1,14)=14.6, p=.002, M S e=0.28$, as N1 was more pronounced on incongruent trials $(-0.32 \mu \mathrm{V})$ than on congruent trials $(-0.06 \mu \mathrm{V})$. In addition, there was a significant Electrode Side x Probe Side interaction, $F(1,14)=35.9, p<.001, M S e$ $=4.9$, as $\mathrm{N} 1$ was more pronounced at contralateral sites than at ipsilateral sites. This was modulated by cues, as indicated by a Electrode Side x Probe Side x Cued Side interaction, $F(1,14)=5.2, p=.039, M S e=0.05$.

A

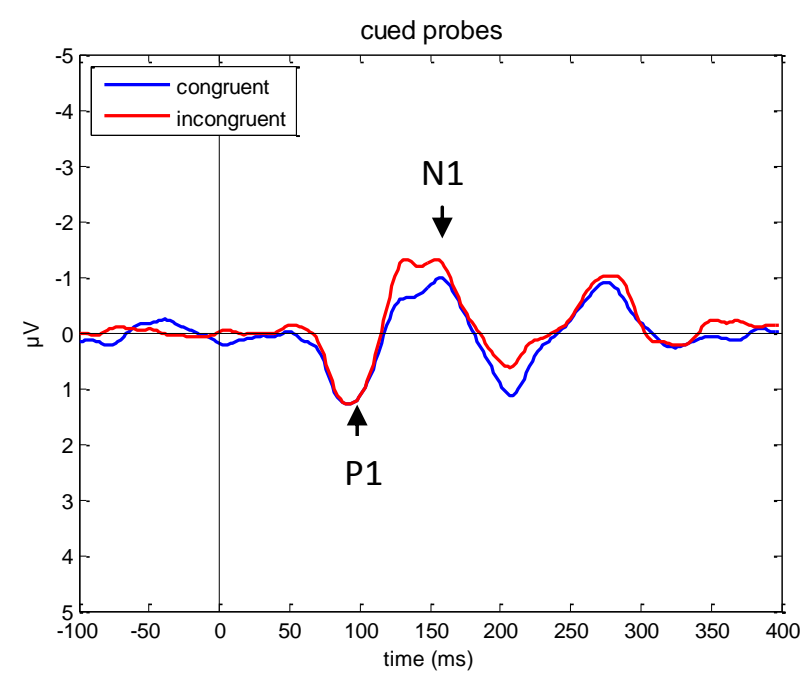

B

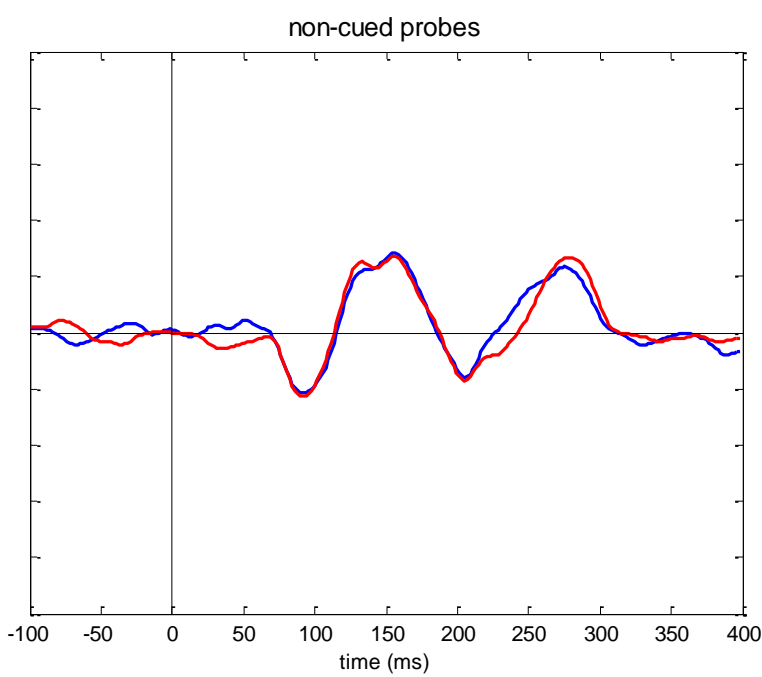

Figure 4.10 Probe - no probe difference potentials at PO7/PO8 for (A) cued contralateral probes and (B) noncued contralateral probes for congruent and incongruent trials. Potentials for trials without probe were subtracted from waveforms with probe to obtain potentials associated with probe processing. 


\section{Contralateral analysis of probe potentials}

In an additional analysis, we focused only on probe related ERPs induced at contralateral sites, because interpretation of the probe-no probe difference was made difficult for ipsilateral sites, as P1 and N1 were smaller there than when the probe was absent. Figure 4.10 shows probe-no probe difference waves at PO7/PO8 only for contralateral probes.

P1. P1 was enhanced on congruent trials at PO7 but diminished at PO8 resulting in a significant Electrode Side x Congruency interaction, $F(1,14)=5.2, p=.038, M S e=0.4$. In

A

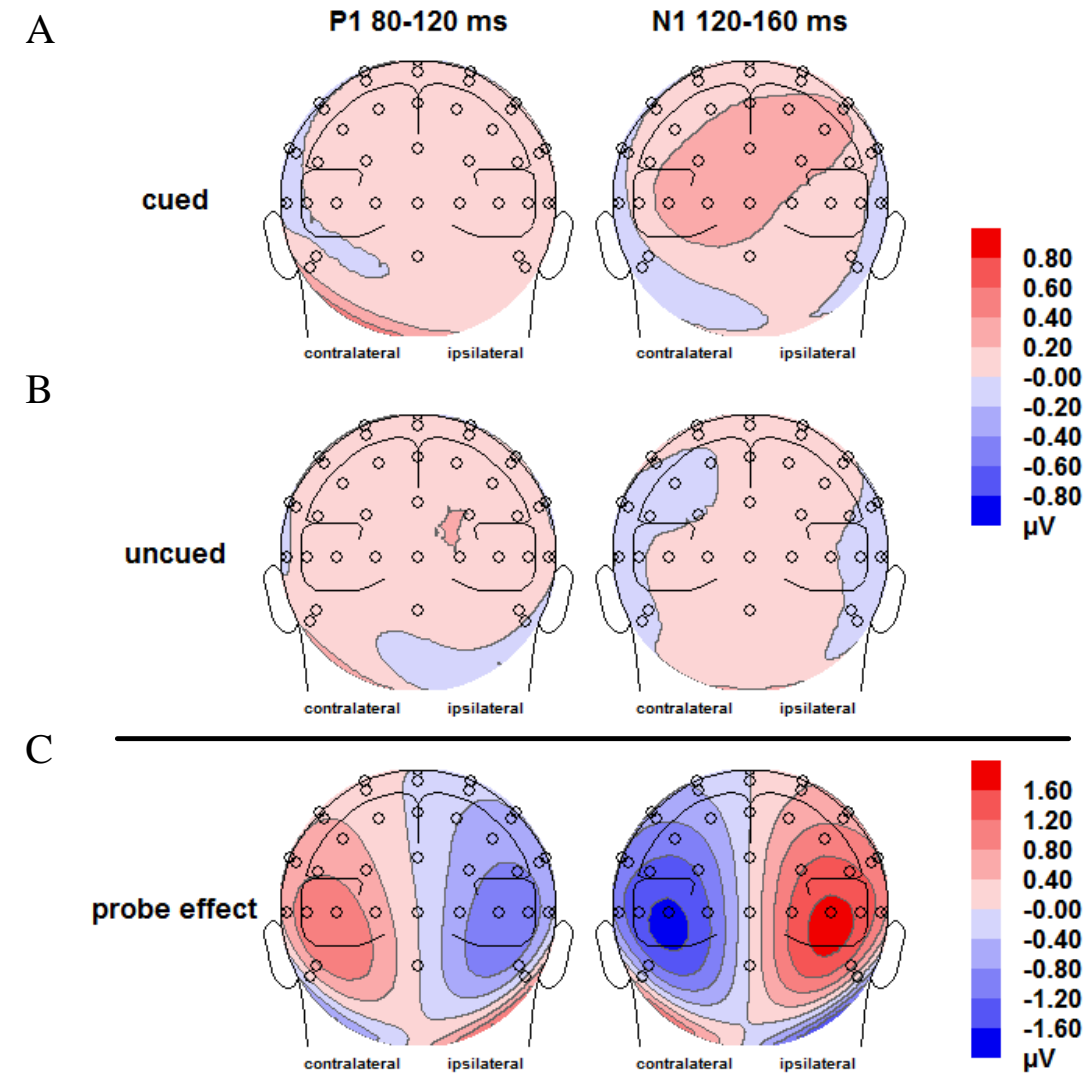

N1 120-160 ms

Figure 4.11 Voltage maps for analyzed periods in the probe - no probe difference in Experiment 9. ERP data were collapsed over probe side (left, right) and recording hemisphere (left, right) to show ipsilateral and contralateral ERP distributions on the left and right sides of the maps, respectively (A) Congruency effect (congruent-incongruent) on trials with cued probe. (B) Congruency effect on trials with non-cued probe. (C) Probe effect (probe - no probe) averaged across all trials. 
addition, we found a significant Electrode Side x Cued Side interaction, $F(1,14)=6, p=$ $.028, M S e=0.2$, indicating that $\mathrm{P} 1$ was larger when the contralateral side was cued $(0.84 \mu \mathrm{V})$ than when the ipsilateral side was cued $(0.65 \mu \mathrm{V})$.

N1. Contralaterally, N1 was more negative on incongruent trials $(-1.2 \mu \mathrm{V})$ than on congruent trials $(-0.9 \mu \mathrm{V}), F(1,14)=11.8, p=.004, M S e=0.2$. In addition, there was a significant Electrode Side x Cued Side x Congruency Interaction, $F(1,14)=5.8, p=.029$, $M S e=0.3$, indicating that this priming effect on N1 was larger when the probe appeared at the cued side $(-0.51 \mu \mathrm{V})$ than when it appeared at the non-cued side $(-0.06 \mu \mathrm{V})$.

To summarize, there were some effects on probe related P1 and N1 amplitudes, but these components were not found to be modulated by Congruency in the predicted way. Instead, N1 for cued probes was even enhanced by incongruent primes.

\section{Target-locked ERPs}

Several components of target-locked potentials (Figure 4.12) were analyzed using only
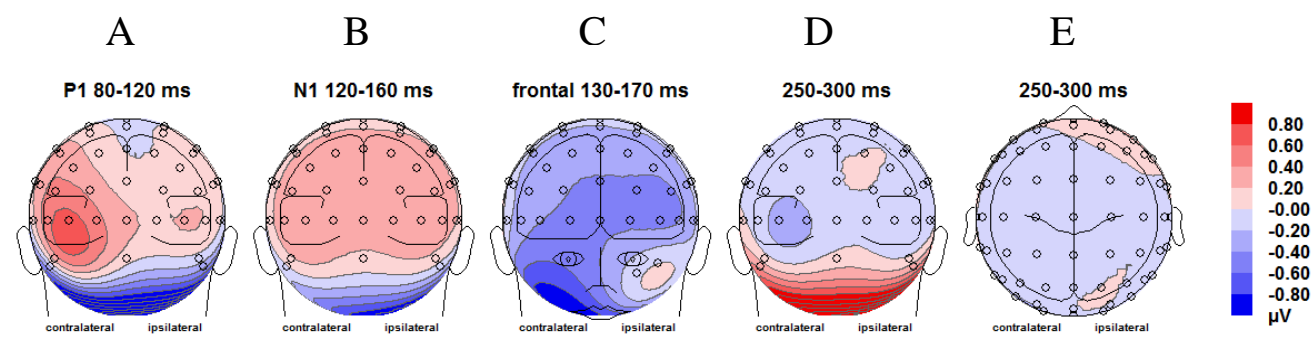

Figure 4.12 Voltage maps for priming effects (congruent-incongruent) in analyzed periods in target-locked ERPs in Experiment 9. ERP data were collapsed over cued side (left, right) and recording hemisphere (left, right) to show ipsilateral and contralateral ERP distributions on the left and right sides of the maps, respectively. (A) occipital distribution of the P1 priming effect. (B) Occipital distribution of the N1 priming effect. (C) Frontal distribution of priming effect on a negative component. (D) Occipital distribution of the P3 priming effect. (E) central distribution of the $\mathrm{P} 3$ priming effect. 
trials without probes to exclude any effects of probe presentation. Due to the presentation of cues shortly in advance, target-locked ERPs at PO7/PO8 (Figure 4.13) did not exhibit the typical time course. Consequently, P1 and N1 could not be unambiguously defined. Therefore, we used similar time windows as in Experiment 8 to assess priming effects on target P1 (80-140 ms) and N1 (140-200 ms).

$P 1$. For the P1 period we found a main effect of Electrode Side, $F(1,14)=9.2, p=$ $.009, M S e=2.7$, with increased P1 at PO8 $(0.56 \mu \mathrm{V})$ compared to PO7 $(-0.35 \mu \mathrm{V})$. A main effect of Cued Side, $F(1,14)=19.6, p<.001, M S e=1.4$, revealed that P1 was increased when the right side was cued $(0.58 \mu \mathrm{V})$ compared to when the left side was cued $(-0.38 \mu \mathrm{V})$. More importantly, we found a main effect of Congruency, $F(1,14)=15, p=.002, M S e=1.0$, as $\mathrm{P} 1$ was more positive on congruent trials $(0.45 \mu \mathrm{V})$ than on incongruent trials $(-0.25 \mu \mathrm{V})$. A significant Electrode Side x Cued Side x Congruency interaction, $F(1,14)=7.2, p=.018$, $M S e=0.46$, revealed that this trend was not apparent at PO8 when the right side was cued

A

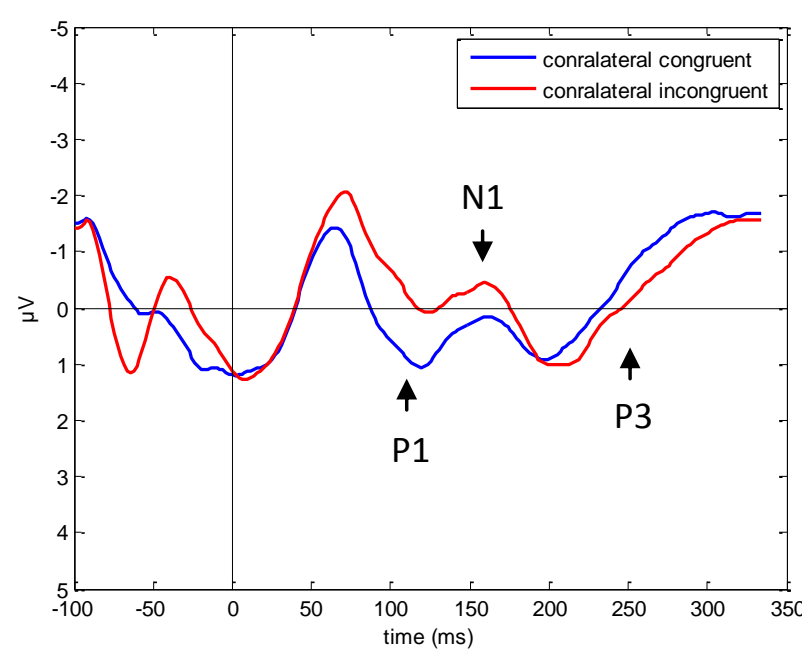

$\mathrm{B}$

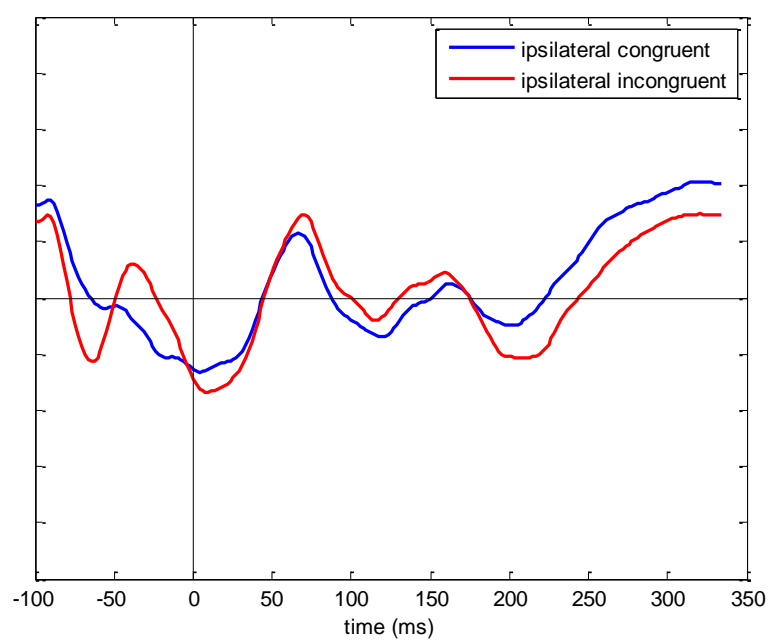

Figure 4.13 Target-locked ERPs at PO7/PO8 for congruent and incongruent no probe trials. (A) ERPs for contralateral targets. (B) ERPs for ipsilateral targets. 
(difference congruent-incongruent: $-0.5 \mu \mathrm{V}$ ), but with the other three combinations of Cued Side and Electrode Side (difference $>0.6 \mu \mathrm{V}$ ). This also resulted in a significant Electrode Side x Congruency interaction, $F(1,14)=6.6, p=.022, M S e=0.24$ (difference left: $0.93 \mu \mathrm{V}$; right $0.47 \mu \mathrm{V})$, and a Cued Side $\mathrm{x}$ Congruency interaction, $F(1,14)=9.3, p=.009, \mathrm{MSe}=$ 1.3 (difference left: $1.34 \mu \mathrm{V}$; right $0.06 \mu \mathrm{V}$ ).

N1. During the N1 period, there was no significant main effect of congruency, $F<0.5$ $p>.5$. However, Congruency interacted significantly with both Electrode Side, $F(1,14)=$ 7.4, $p=.017, M S e=0.15$ and Cued Side, $F(1,14)=34.7, p<.001, M S e=2$. There was a priming effect on $\mathrm{N} 1$ defined as the congruent-incongruent difference at $\mathrm{PO} 7(0.39 \mu \mathrm{V})$ but not at PO8 $(-0.002 \mu \mathrm{V})$. Priming effects differed in direction between left cues $(1.7 \mu \mathrm{V})$ and right cues $(-1.3 \mu \mathrm{V})$. Note, that negative priming effects indicate larger amplitudes on incongruent trials and would be expected if $\mathrm{N} 1$ is increased on congruent trials. In addition, there were main effects of Electrode Side, $F(1,14)=7.6, p=.016, M S e=5.7$, indicating more positive amplitude at PO8 $(0.78 \mu \mathrm{V})$ than at PO7 $(-0.42 \mu \mathrm{V})$, and Cued Side, $F(1,14)=$ $59.9, p<.001, M S e=0.9$, indicating more positive amplitude when the right side was cued $(0.84 \mu \mathrm{V})$ than when the left side was cued $(-0.49 \mu \mathrm{V})$.

Note that this classification of potential shifts as target P1 and N1 is problematic for targets, because the relevant periods might be contaminated by differences caused by primes and cues. The same is true for effects of Congruency outside of occipital P1 and N1 reported in the following.

Frontal negativity. Frontally (averaged over FP1 and FP2, Figures 4.12, 4.14), an effect of congruency emerged during a period that might correspond to the target N1, but could also be interpreted as elicited by prime and cue. From 130 to $170 \mathrm{~ms}$ after target presentation, a negative deflection was more pronounced on congruent trials $(-1.14 \mu \mathrm{V})$ than 
on incongruent trials $(-0.57 \mu \mathrm{V}), F(1,14)=6.9, p=.020, M S e=1.4$. This priming effect, defined as the difference between congruent and incongruent trials, was only apparent when the left side was cued $(-1.19 \mu \mathrm{V})$, but not when the right side was cued $(0.05 \mu \mathrm{V})$, which resulted in a significant Cued Side $\mathrm{x}$ Congruency interaction, $F(1,14)=7.1, p=.018, M S e=$ 1.6

P3. There were congruency effects during a later time window, which can be interpreted as modulation of a P3 component (250-300 ms). This effect was widespread (Figure (4.12D-E) and was most prominent at left occipital and central electrodes. In an analysis of FC3/FC4 (Figure 4.15A), which was done to assess the more frontal aspect of this component, there was a marginal main effect of congruency, $F(1,14)=4.4, p=.055, M S e=$ 2.1, and marginal Electrode Side x Congruency interaction, $F(1,14)=3.4, p=.088, \mathrm{MSe}=$ 0.2. Separate ANOVAs for the two electrodes revealed that Congruency significantly affected amplitudes at FC3, $F(1,14)=7.7, p=.015, M S e=1$, as amplitude was increased on

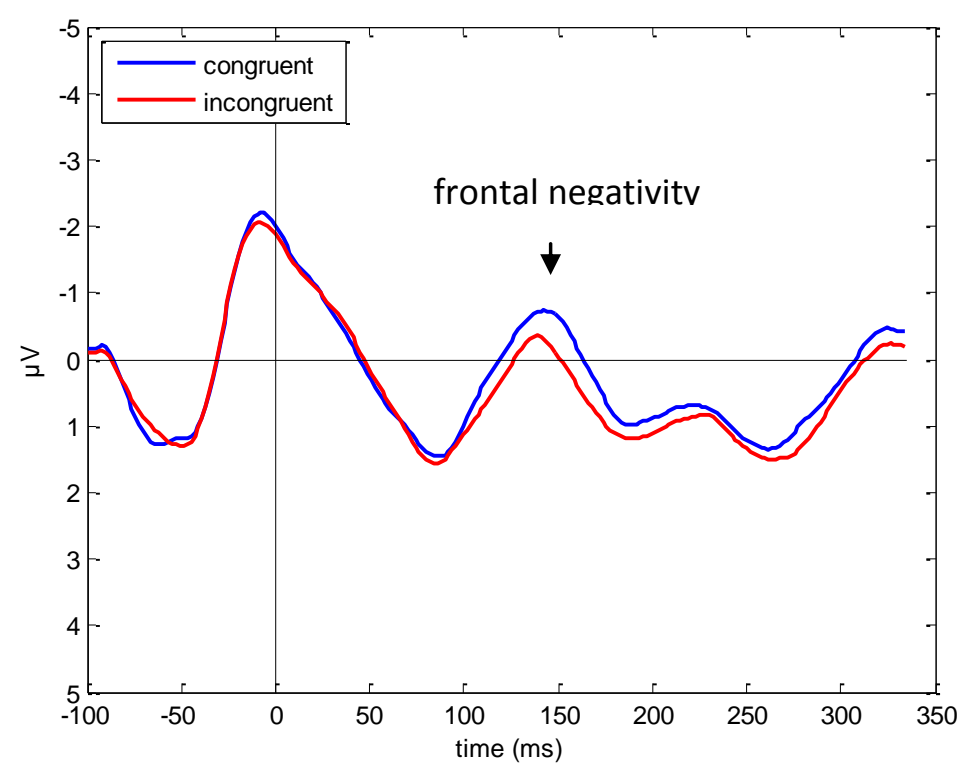

Figure 4.14 Target-locked potentials at FP1/FP2 for congruent and incongruent trials. 


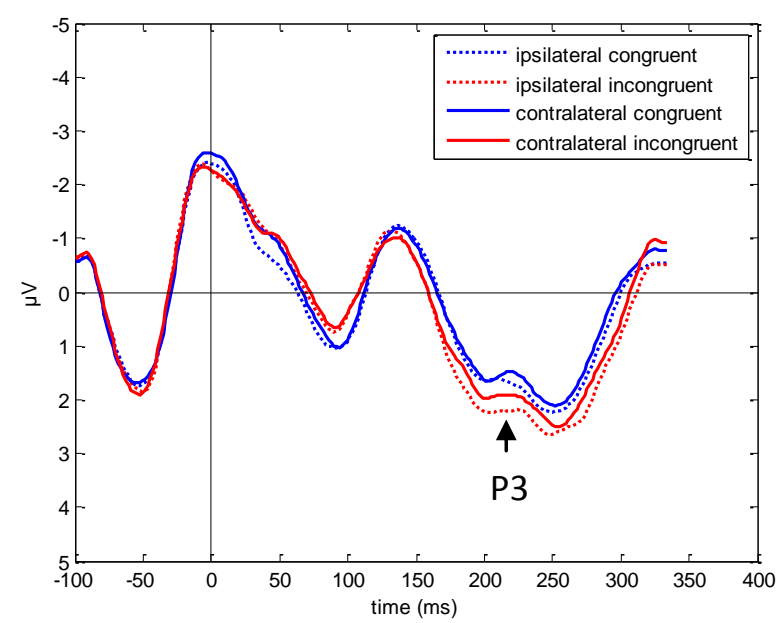

Figure 4.15 Target-locked potentials at FC3/FC4 for congruent and incongruent no probe trials separated for contralateral and ipsilateral targets.

incongruent trials $(2.0 \mu \mathrm{V})$ compared to congruent trials $(1.3 \mu \mathrm{V})$, whereas this difference was not significant at FC4, $F<2, p>.2$; difference on congruent trials: $1.3 \mu \mathrm{V}$, difference on incongruent trials: $1.7 \mu \mathrm{V}$.

At lateral occipital electrodes (PO7/PO8, Figure 4.13), amplitude was also larger for incongruent than for congruent trials, as there was a main effect of congruency, $F(1,14)=$ $6.2, p=.026, M S e=1.8$. However, this was only the case when the right side was cued (priming effect: $-1.79 \mu \mathrm{V})$, but not when the left side was cued $(0.59 \mu \mathrm{V})$, as indicated by a Cued Side x Congruency interaction, $F(1,14)=23.2, p<.001, M S e=1.8$. There was also a main effect of cued side, $F(1,14)=8.5, p=.011, M S e=1.5$. These large effects of Cued Side make it seem probable that congruency effects reflect cue processing rather than target processing.

\subsubsection{Discussion}

Probe-evoked potentials were not modulated by cues, as there were no differences in early visual potentials between probes shown at the cued side and probes shown on the other 
side. In addition, we did not find the expected priming effects on probe evoked potentials. Instead, N1 was enhanced for probes appearing on the cued side on incongruent compared to congruent trials. If probes are thought of as additional peripheral cues, congruent trials with the probe appearing at the cued side are special in that there is no conflict between directing attention according to the prime, the cue, or the probe, as they all suggest the same side. Early ERP studies of sustained attention (Heinze et al., 1990; Luck et al., 1990) related N1 modulation to attentional reorienting to unilateral stimuli. This reorientation might not be needed in this case of complete congruency.

Participants reported that they were able to ignore the probes and did not feel distracted by their presence. However, behavioral data suggest that participants were not able to completely ignore the probes, because probes substantially affected RT. Nevertheless, ignoring the probes as much as possible might be beneficial to performance, as they have no predictive value. Interestingly, P1 and N1 were smaller when a probe was presented ipsilaterally than when the probe was omitted. This might hint at inhibitory processes.

One reason why probe P1 and N1 were unaffected by attention in Experiment 9 could be that cue-target SOA in Experiment 9 was too short for attentional resources to be allocated in time. Luck et al., (1996) proposed that resource allocation might take more time than other attentional processes, such as uncertainty reduction. An additional explanation for the lack of priming and cueing effects on probe potentials could be the lack of perceptual load in Experiment 9. Lavie and Tsal (1994; see also Luck \& Hillyard, 2000) proposed that the locus of attentional selection depends on task demands and that selection operates at early perceptual stages when perceptual load is high. Perceptual load in Experiment 9 was probably lower than in Experiment 8, because perceptual differences between A and B are much more pronounced than between the two different bar targets in Experiment 8. Thus, it might be that 
attention in the letter task used in Experiment 9 operates at later stages than in Experiment 8, and its effects are therefore not apparent in early visual potentials. This view is supported by a study by Handy \& Mangun (2000), who found differences in P1 and N1 modulation depending on the amount of perceptual load.

It might be that the selection of SOAs in Experiment 9 was not ideally suited to detect priming effects on processing of stimuli presented at the non-cued side. The short cue-target SOA was chosen because behavioral priming effects in Experiment 1 were largest with short cue-target SOAs. We reasoned that effects of attentional allocation are likely to be largest shortly after prime presentation, because effects of the primes become negated by the cues at later points in time. However, from Experiment 8 as well as Project 2 it seems that attentional effects on early levels of processing are larger with longer cue-target SOAs, most likely because it takes time to allocate processing resources on early levels of processing (Luck et al., 1996). There might be only a small time window during which effects of resource allocation to processing at the non-cued position caused by incongruent primes can be measured before they are overwritten by cues. Thus, future attempts to study cue-priming effects on stimuli presented at non-cued positions should include variation of cue-target SOA in order to find this time window.

In additional analyses, we found priming effects that could be interpreted as a modulation of early visual potentials elicited by target stimuli. A similar pattern as observed here with increased positivity in the range of target P1 and - in the present experiment to a lesser degree - N1 has been found in previous studies which combined bilateral targets with involuntary auditory cueing (McDonald, Teder-Sälejärvi, Di Russo \& Hillyard, 2005; Störmer, McDonald \& Hillyard 2005). Due to the fact that Experiment 9 was mainly designed to investigate probe related ERPs, interpretation of priming effects on target-locked potentials 
suffers from confoundation with differences in potentials evoked by primes and cues between congruent trials and incongruent trials. Therefore, this result cannot be taken as evidence for priming effects on target processing. Likewise, later effects at frontal and central electrodes might reflect cue processing instead of target processing. In a task switching study, Periáñez \& Barceló (2009) found a cue-locked frontocentral N2 component in a time window similar to the frontal negative going component found here, which was enhanced on cue repeat trials compared to cue switch trials. They speculated that this might reflect sensory priming of cue encoding. A similar effect has been reported by Nicholson et al. (2006) who found an increased N2 when subsequent cues came from the same category versus when there was a switch in cue category. Thus, the modulation of frontal negativity by Congruency in Experiment 9 might be a result of primes priming cue encoding. Later Congruency effects on ERPs were found at lateral occipital and parietal electrodes. It is again unclear whether this is indicative of priming effects on target processing, priming effects on cue processing, or both. It might also be that this difference reflects some kind of response preparation. This effect might also relate to results of Periáñez \& Barceló (2009) who found enhanced cue-locked P3 amplitudes from 300-340 ms over centro-parietal regions following cue- and task-switches. This time window corresponds to the one analyzed here. Importantly, these late effects were not present in Experiment 8. This might suggest that primes affected different processes in Experiment 9 than in Experiment 8. These priming effects on late potentials stand in contrast to the results of Experiment 8. If they are indeed indicative of priming of cue processing, these effects might suggest that the increased perceptual similarity between prime and cue due to both being presented at the same location enabled additional priming effects. Alternatively, these late components might reflect selection processes which are needed to decide between the two competing letters. Such a decision was not necessary in Experiment 8. The priming effects on target-locked ERPs showed some inconsistency as some were only found at 
specific electrode sites or when a specific side was cued. These interactions do not seem easily explainable and might further impede interpretation of the priming effects.

In summary, we did not find any evidence that incongruent primes can orient attention to the non-cued side. Further, it seems that priming effects in this letter discrimination task with short cue-target SOA might be based on different processes than in Experiment 8, where a bar discrimination task without distractor and with longer cue-target SOA was employed. It is unclear at this point, whether the crucial difference lies in cue-target SOA or another task parameter, e.g., differences in perceptual load. Interpretation of results beyond this is made difficult by confoundation of cued side with cue symbols and short cue-target SOAs. This makes it impossible to discern whether differences in ERPs are caused by differences in target processing or cue processing. Together with the larger behavioral priming effect here, the modulation of later ERP-components by congruency which was not found in Experiment 8, suggest that priming effects do not entirely result from the same processes in the two experiments. This might be because of differences between the tasks or the employed cue and prime stimuli.

\subsection{Experiment 10 - The role of cue-target SOA and distractor presence}

In Experiment 9, probe processing seemed to be unaffected by primes, even though behavioral priming effects were larger than in Experiment 8. In Experiment 8, we found small priming effects on early target processing that correlated with small behavioral priming effects. Experiment 10 aimed at replicating this effect and clarifying which difference between the experiments accounts for these findings. One possibility is that priming effects on early target processing only emerge with sufficiently long cue-target SOAs because resource allocation takes time (Luck et al., 1994). Large behavioral effects at short cue-target SOAs such as in Experiment 9 could be based on later selection processes and be independent of 
early target processing. Attentional selection could operate at different stages in the two experiments because of different task demands induced by differences in cue-target SOA, distractor presence, or perceptual load, which is probably higher with bar targets (Handy \& Mangun, 2000). In Experiment 10, we used the same perceptually demanding task as in Experiment 8 and varied cue-target SOA in two steps. We used one short SOA which corresponds to Experiment 9 and a long SOA similar to Experiment 8. In addition, we varied task demands by presenting a relevant distractor stimulus together with the target in half of the trials. In previous cue-priming experiments, priming effects were unaffected by the presence of a distractor stimulus (Mattler, 2003). However, introducing distractors should change task demands and make them more similar to Experiment 9 and the letter discrimination experiments in Project 1, in which large priming effects were found. Accordingly, they should decrease with cue-target SOA. Behavioral priming effects for the remaining half of the trials (without distractor) should be similar in all conditions and these trials can be used to measure early target processing through P1 and N1 magnitude just like in Experiment 8. Crucially, however, if priming effects on early target processing measured by EEG depend on resource allocation, which takes longer, they should be larger with long cuetarget SOA. Such dissociation would provide evidence that behavioral priming effects in Experiments 8 and 9 are based on different processes. On the other hand, if spatial cuepriming effects are based on differences in early target processing they should decrease with cue-target SOA like behavioral effects. In addition, we used only one location for primes and cues unlike Experiment 9 in case the small effects in Experiment 8 were due to the changes in these stimuli. 


\subsubsection{Method}

Apparatus and Stimuli. Timing and stimuli in a trial of Experiment 10 are shown in Figure 4.16. Primes and cues were squares and diamonds similar to Experiment 9 whereas targets were bright bars of two different lengths similar to Experiment 8. On half of the trials, two bars appeared - one being a target and one a distractor. On these trials, one bar was long and the other was short. Participants had to respond to the stimulus on the cued side. CueTarget SOA was $165 \mathrm{~ms}$ on half of the trials and $400 \mathrm{~ms}$ on the other half of trials. Prime-Cue SOA was $94 \mathrm{~ms}$.

Participants. 13 participants (8 women, mean age 21.9) completed 3 sessions of the experiment. 4 additional participants were excluded from analyses due to too many eye

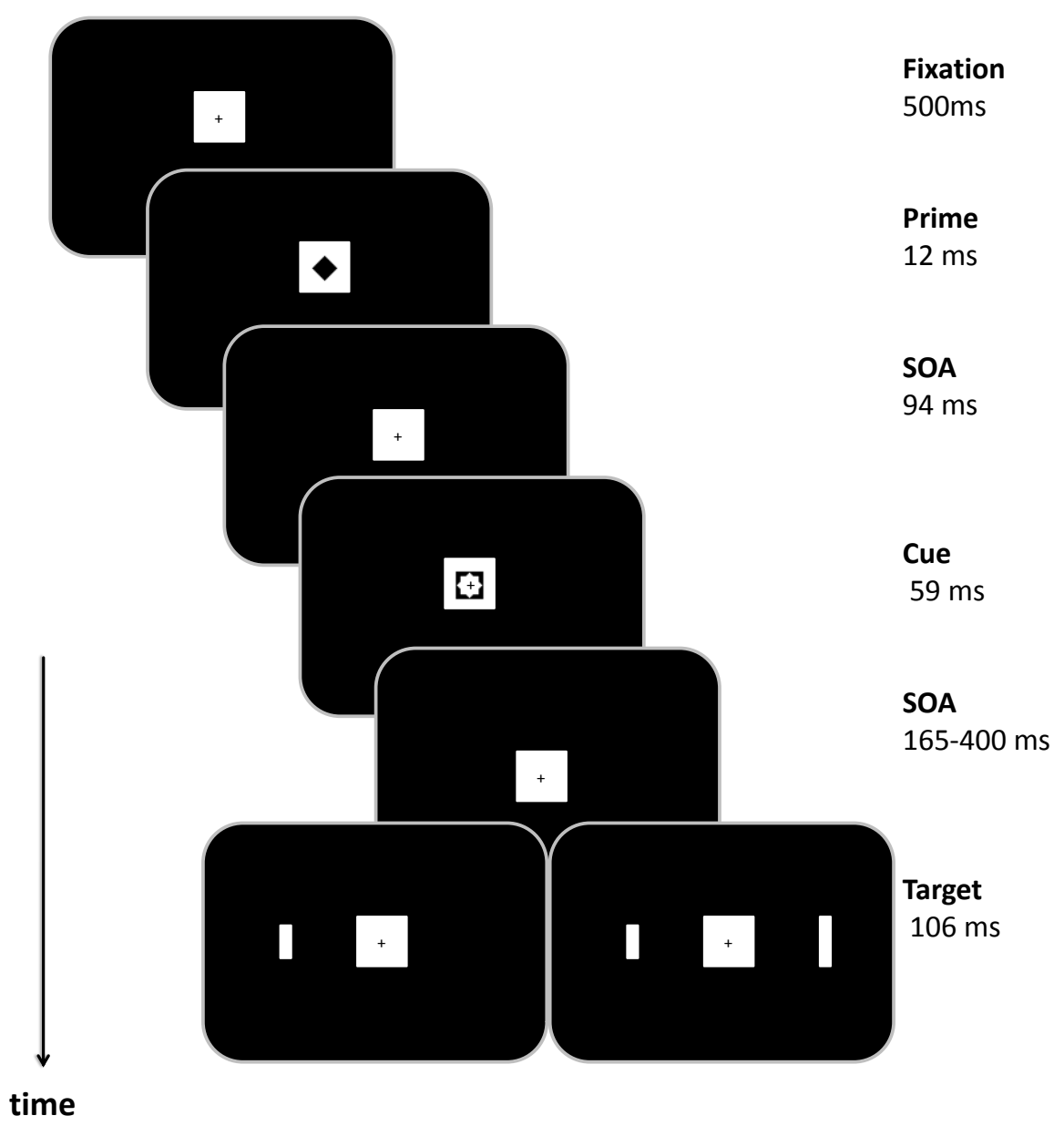

Figure 4.16 Sequence of stimulus events in a trial of Experiment 10. 
movement artifacts. Another 3 participants were excluded during practice session because of problems with eye gaze detection.

Task. Participants had to indicate whether the target on the cued side was long or short by pressing response buttons as fast as possible. The location of the relevant bar was predicted by cues. Square cues predicted a target on the left side; diamond cues predicted a target on the right side.

Design and procedure. Procedure was the same as in Experiment 8. Participants completed a practice session, an EEG session and a prime recognition session. In every block of 64 trials every combination of 2 SOAs, 2 primes, 2 cues, and 2 targets was presented twice with distractor, and twice without distractor.

ERP-Averaging and Analyses. We used the same methods as in the previous experiments to compute ERPs. In order to obtain target potentials for the short SOA which were uncontaminated by primes and cues we subtracted the respective long SOA condition in one analysis. As there were unexpectedly large differences in priming effects as well as in target-locked potentials between long and short SOA conditions (see below) we analyzed ERPs separately for each SOA.

Reported analyses are restricted to lateral occipital electrodes. P1 was defined as average amplitude from 80-140 ms after target onset at PO7 and PO8. N1 was averaged at the same electrodes from 140-180 ms. With $165 \mathrm{~ms}$ SOA there was an effect of Congruency about $300 \mathrm{~ms}$ after target onset which was maximal over central occipital sites. Therefore, we used a window from 280 to $330 \mathrm{~ms}$ at $\mathrm{O} 1$ and $\mathrm{O} 2$ to analyze this component. With $400 \mathrm{~ms}$ SOA visual inspection suggested Congruency effects on an N2 component at PO7/PO8. Mean amplitude from 200-260 ms after target onset was used to analyze this component. 
With short cue-target SOA, target-locked potentials were considerably affected by cue induced shifts of potential. In order to isolate potentials related to target processing from those related to cue processing, we used prime-locked potentials and subtracted long SOA ERP from short SOA ERP. As prime and cue are the same in both conditions all differences between short and long SOA before $495 \mathrm{~ms}$ after prime onset (onset of the target in the 400 ms SOA condition) should result from presentation of the short-SOA target. We used the same time windows for P1 and N1 calculation as in Experiment 8 because no clear P1 and N1 peaks were visible in the difference waveform. Mean amplitudes were analyzed using $2 \times 2 \times$ $2 \times 2$ repeated measure ANOVAs with factors Electrode Side, Cued Side, Distractor Presence, and Congruency.

\subsubsection{Results}

$R T$. Responses were faster on trials without distractor $(613 \mathrm{~ms})$ than on trials with distractor $(716 \mathrm{~ms}), F(1,12)=31.2, M S e=8752, p<.001$ (Figure 4.17A-B). In addition, participants responded faster on trials with long SOA $(617 \mathrm{~ms})$ than on trials with short SOA (711 ms), $F(1,12)=130, M S e=1763, p<.001$. We also found a significant interaction between Distractor Presence and SOA, $F(1,12)=10, M S e=448, p=.008$, indicating that the presence of a distractor had larger effects with short SOA (116 ms) than with long SOA (81 ms). There was no significant main effect of Congruency on RT, $F<1, p>.4$, but a significant Congruency x SOA interaction $F(1,12)=7.9, M S e=4158, p<.016$. In order to assess this interaction, we performed an additional ANOVA for each level of SOA. We found that with short SOA responses were faster on congruent trials $(704 \mathrm{~ms})$ than on incongruent trials $(719 \mathrm{~ms}), F(1,12)=5.6, M S e=531, p=.036$. However, with long SOA the opposite was true and responses were slower on congruent trials $(623 \mathrm{~ms})$ than on incongruent trials 
$(613 \mathrm{~ms}), F(1,12)=5.8, M S e=230, p=.033$. The Distractor Presence x Congruency interaction did not reach significance for either SOA $(F<2, p>.2$, in both cases).

Prime recognition. Prime recognition performance did not differ between SOAs, $t(12)$ $=1, p=0.347$, and was above chance level (mean $d^{\prime}=1.4, t=4.9, p<.001$ ). Across participants, recognition performance did not correlate with priming effects on RT in any condition, $-0.3<r<0.1, p>.35$, in all cases.

Error rates. Participants committed more errors on trials with short SOA (15.9\%) than on trials with long $\operatorname{SOA}(12.7 \%), F(1,12)=26, M S e=0.009, p<.001$ (Figure 4.17C-D). There was a marginal effect of Distractor Presence, $F(1,12)=4.3, M S e=0.064, p=.059$,
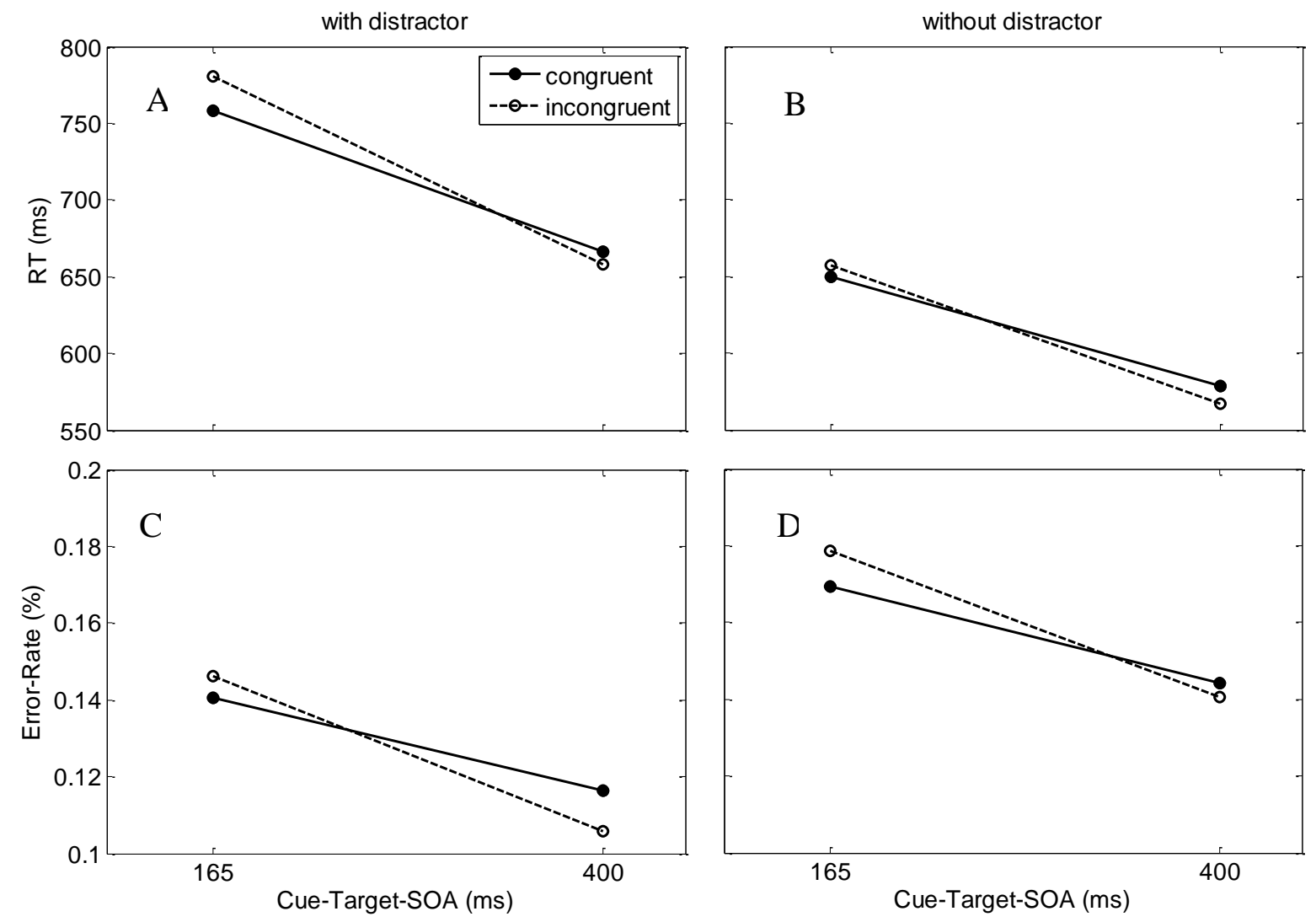

Figure 4.17 RT (top panels, A-B) and error rates (bottom panels, C-D) for trials with distractor (left panels) and trials without distractor (right panels). 
indicating that responses tended to be more error prone on trials without distractor $(15.8 \%)$ than on trials with distractor $(12.7 \%)$. There was no significant main effect of Congruency, $F$ $<0.1, p>.9$, nor did the Congruency x SOA interaction reach significance, $F<2, p=.2$.

\section{ERP analyses}

Overall, results were similar for trials with and trials without distractor, with the exception that distractors induced larger visual potentials at electrodes ipsilateral to the cued side (Figure 4.18).

\section{Short SOA}

ERPs for short SOA trials are given in Figure 4.19.

P1. There was a main effect of Cued Side, $F(1,12)=8.2, p=.014, M S e=2.0$, indicating that P1 was larger when the right side was cued $(1.26 \mu \mathrm{V})$ than when the left side

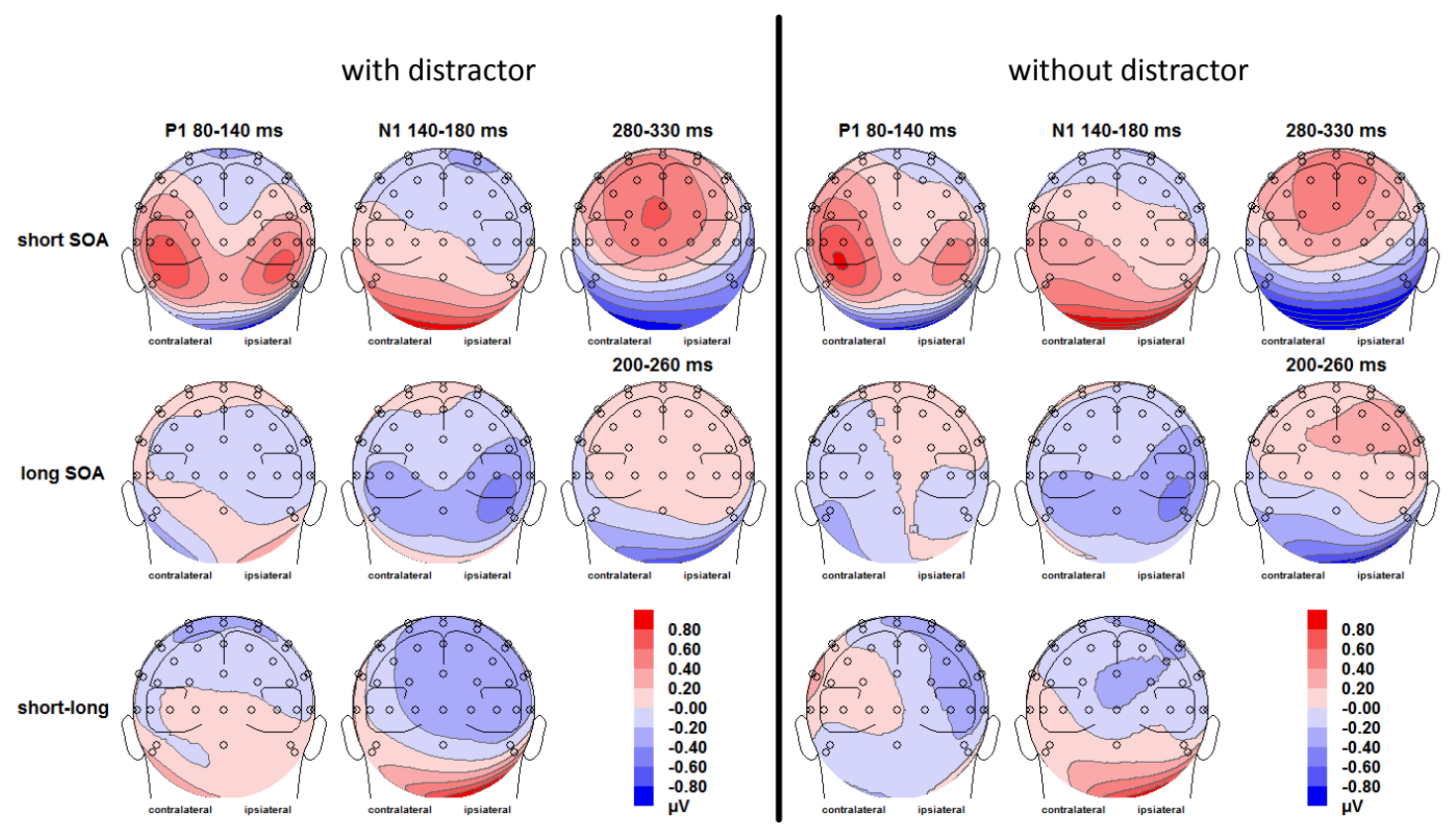

Figure 4.18 Voltage maps for congruency effects (congruent-incongruent) in analyzed periods in Experiment 10. ERP data were collapsed over cued side (left, right) and recording hemisphere (left, right) to show ipsilateral and contralateral ERP distributions on the left and right sides of the maps, respectively. 

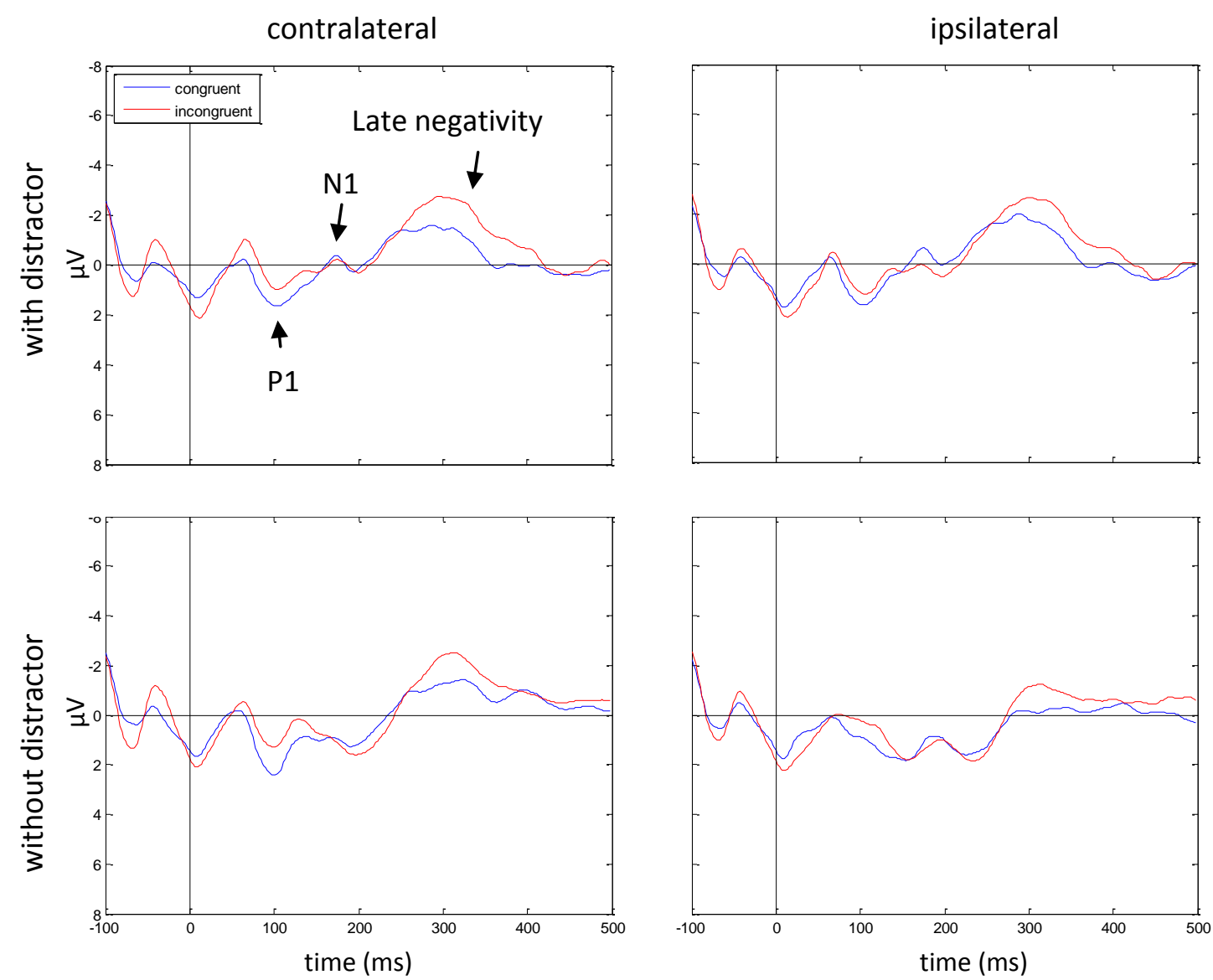

Figure 4.19 Target-locked ERPs at PO7/PO8 for congruent and incongruent trials with short SOA. ERPs were collapsed over left- and right-cue conditions and left and right hemispheres to obtain waveforms recorded and contralaterally (left panels) and ipsilaterally (right panels) to the side of the cue. Separated for trials with distractor (top panels), and trials without distractor (bottom panels).

was cued $(0.7 \mu \mathrm{V})$. P1 was significantly enlarged on congruent trials $(1.33 \mu \mathrm{V})$ compared to incongruent trials $(0.63 \mu \mathrm{V}), F(1,12)=8.3, p=.014, M S e=3.1$. However, a significant Cued Side $\mathrm{x}$ Congruency interaction, $F(1,12)=12.3, p=.004, M S e=2.2$, revealed that this was only the case when the left side was cued (priming effect: $1.42 \mu \mathrm{V}$ ) but not when the right side was cued (priming effect: $-0.02 \mu \mathrm{V}$ ). In addition, the priming effect was affected by Electrode Side, as indicated by a significant Congruency x Electrode Side interaction, $F(1$, $12)=5.7, p=.034, M S e=0.26$ The difference between congruent and incongruent trials was $0.87 \mu \mathrm{V}$ at PO7 but only $0.53 \mu \mathrm{V}$ at PO8. 
N1. For a later period that was previously used to assess N1 amplitudes, a main effect of Distractor Presence, $F(1,12)=21, p=.001, M S e=3.4$, indicated that overall amplitudes where higher on trials without distractor $(1.24 \mu \mathrm{V})$ than on trials with distractor $(0.07 \mu \mathrm{V})$. In addition, there was a main effect of Cued Side, $F(1,12)=29.4, p<.001, M S e=1.7$, which indicated that in this period mean amplitude was higher when the right side was cued (1.15 $\mu \mathrm{V})$ than when the left side was cued $(0.16 \mu \mathrm{V})$. There was no main effect of Congruency $(F$ $<1, p>.8$ ), but a significant Electrode Side x Congruency interaction, $F(1,12)=6.2, p=$ $.028, M S e=0.71$, as well as a Cued Side $\mathrm{x}$ Congruency interaction, $F(1,12)=14.7, p=.002$, $M S e=4.6$. Numerically, N1 amplitude during this period was larger on congruent than on incongruent trials at PO8 (priming effect: $-0.34 \mu \mathrm{V}$ ), but not at PO7 $(0.24 \mu \mathrm{V})$, and when the right side was cued $(-1.19 \mu \mathrm{V})$, but not when the left side was cued $(1.09 \mu \mathrm{V})$. Note that negative values for priming effects again indicate larger N1 on congruent trials.

Late negativity (280-330 ms). This late component was analyzed at $\mathrm{O} 1 / \mathrm{O} 2$ because visual inspection suggested a maximal difference there (Figure 4.18, top right, both with and without distractor). ERPs for these electrodes are not shown because the presence of the priming effects can also be seen in the ERPs for the more lateral PO7/PO8 electrodes (Figure 4.19). During this period, mean amplitude was more negative on incongruent trials $(-2.14 \mu \mathrm{V})$ than on congruent trials $(-0.97 \mu \mathrm{V}), F(1,12)=28.9, p<.001, M S e=2.5$. There also was a marginal Cued Side x Congruency interaction, $F(1,12)=4.7, p<.052, M S e=3.3$, as priming effects tended to be larger when the left side was cued $(1.71 \mu \mathrm{V})$ than when the right side was cued $(0.62 \mu \mathrm{V})$. In addition, a significant Electrode Side $\mathrm{x}$ Cued Side $\mathrm{x}$ Congruency interaction, $F(1,12)=7.4, p<.019, M S e=0.08$, indicated that this pattern was more pronounced at $\mathrm{O} 2$ than at O1. An Electrode Side x Cued Side x Distractor Presence interaction, $F(1,12)=8.9, p<.011, M S e=0.84$, resulted from the fact that Distractor 
Presence led to more negative amplitudes at the electrode ipsilateral to the cued side, but not at the electrode contralateral to the cued side. Interestingly, we found a Cued Side $\mathrm{x}$ Congruency x Distractor Presence interaction, $F(1,12)=5.2, p<.042, M S e=0.52$, indicating that with right targets priming effects were larger with distractor than without distractor.

\section{Long SOA}

ERPs for long SOA trials are given in Figure 4.20.
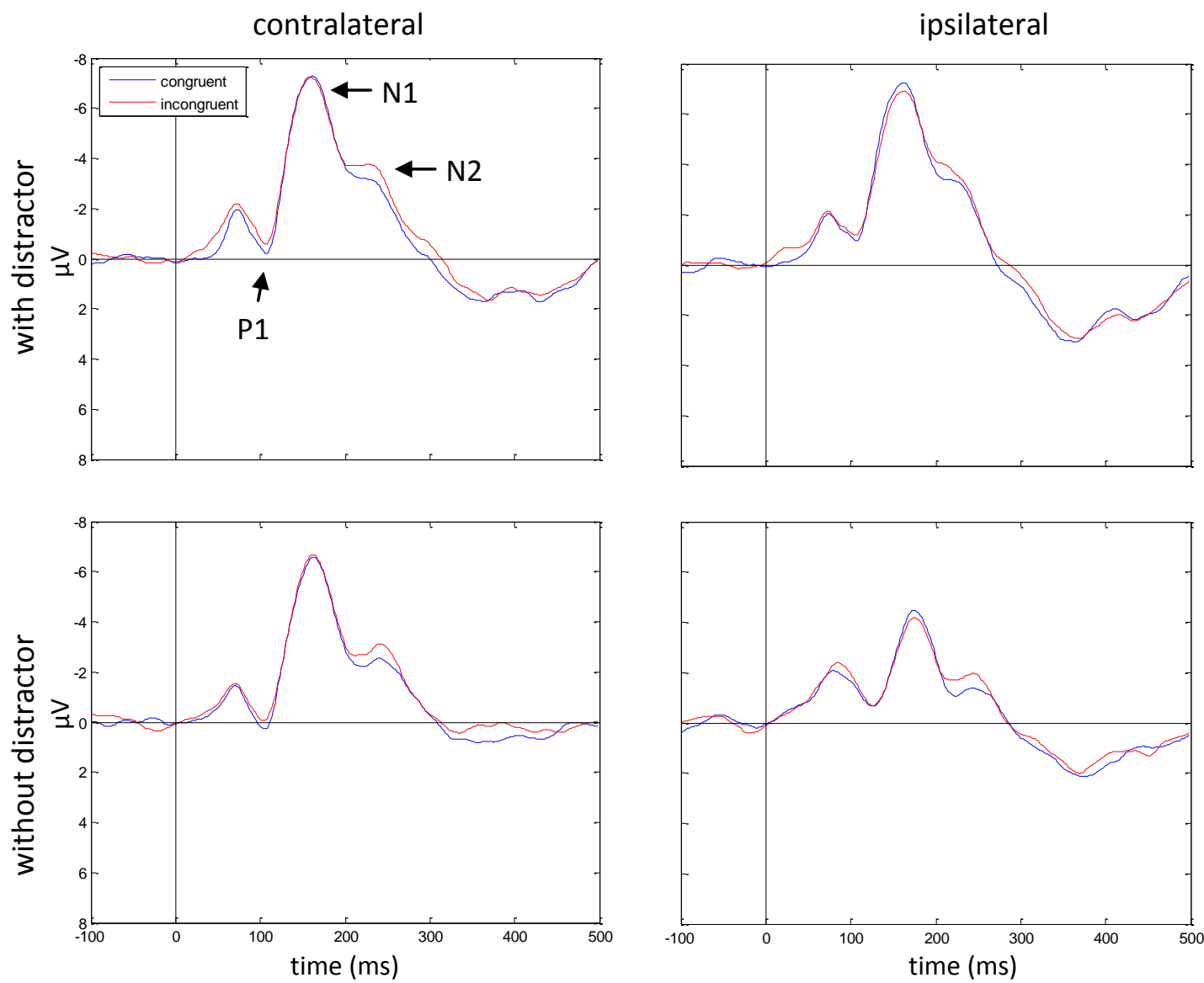

Figure 4.20 Target-locked ERPs at PO7/PO8 for congruent and incongruent trials with long SOA. ERPs were collapsed over left- and right-cue conditions and left and right hemispheres to obtain waveforms recorded and contralaterally (left panels) and ipsilaterally (right panels) to the side of the cue. Separated for trials with distractor (top panels), and trials without distractor (bottom panels). 
$P 1$. With $400 \mathrm{~ms}$ cue-target SOA, there was a main effect of Cued Side, $F(1,12)=$ $14.9, p=.003, M S e=5.6$, which was indicative of the fact that P1 was larger when the left side was cued $(-1.11 \mu \mathrm{V})$ than when the right side was cued $(-2.34 \mu \mathrm{V})$. This was also modulated by Congruency as evidenced by a Cued Side x Congruency interaction, $F(1,12)=$ $14, p=.003, M S e=1.5$, as there was a positive priming effect when the right side was cued $(0.8 \mu \mathrm{V})$, but a negative difference when the left side was cued $(-0.48 \mu \mathrm{V})$. In addition, a main effect of Distractor, $F(1,12)=21.7, p<.001, M S e=1.5$, revealed an overall smaller P1 with distractor $(-2.13 \mu \mathrm{V})$ than without distractor $(-1.33 \mu \mathrm{V})$.

N1. There was a main effect of Cued Side, $F(1,12)=8.8, p=.012, M S e=10$, as N1 was larger when the right side was cued $(-6.3 \mu \mathrm{V})$ than when the left side was cued $(-5 \mu \mathrm{V})$. $\mathrm{N} 1$ was larger with distractor $(-6.68 \mu \mathrm{V})$ than without distractor $-4.63 \mu \mathrm{V}, F(1,12)=68.6, p$ $<.001, M S e=3.2$. A Cued Side x Electrode Side interaction, $F(1,12)=40.5, p<.001, M S e=$ 2.7, was indicative of the fact that $\mathrm{N} 1$ was enlarged contralaterally $(-6.38 \mu \mathrm{V})$ compared to the ipsilateral electrode $(-4.93 \mu \mathrm{V})$. In addition, there was a Cued Side x Congruency interaction, $F(1,12)=16.8, p=.001, M S e=2.4$, as N1 was enlarged by incongruent primes with left targets (difference congruent-incongruent: $-0.99 \mu \mathrm{V}$ ), but diminished for right targets (difference: $0.78 \mu \mathrm{V}$ ). An Electrode Side x Cued Side x Distractor interaction, $F(1,12)=$ $39.5, p<.001, M S e=3.1$, indicated that $\mathrm{N} 1$ was increased in the presence of a distractor at electrodes ipsilateral to the cued side (difference $3.38 \mu \mathrm{V}$ ), but not at contralateral electrode (difference $0.72 \mu \mathrm{V}$ ).

N2. With long SOA there was an occipital (PO7/PO8) effect of congruency in a period from 200 to $260 \mathrm{~ms}$ after target onset, $F(1,12)=5.5, p=.036, M S e=1.7$. This was qualified by a Cued Side x Congruency interaction, $F(1,12)=9.2, p=.0 .11, M S e=1$, indicating that amplitudes were more positive on congruent than on incongruent trials with right targets but 

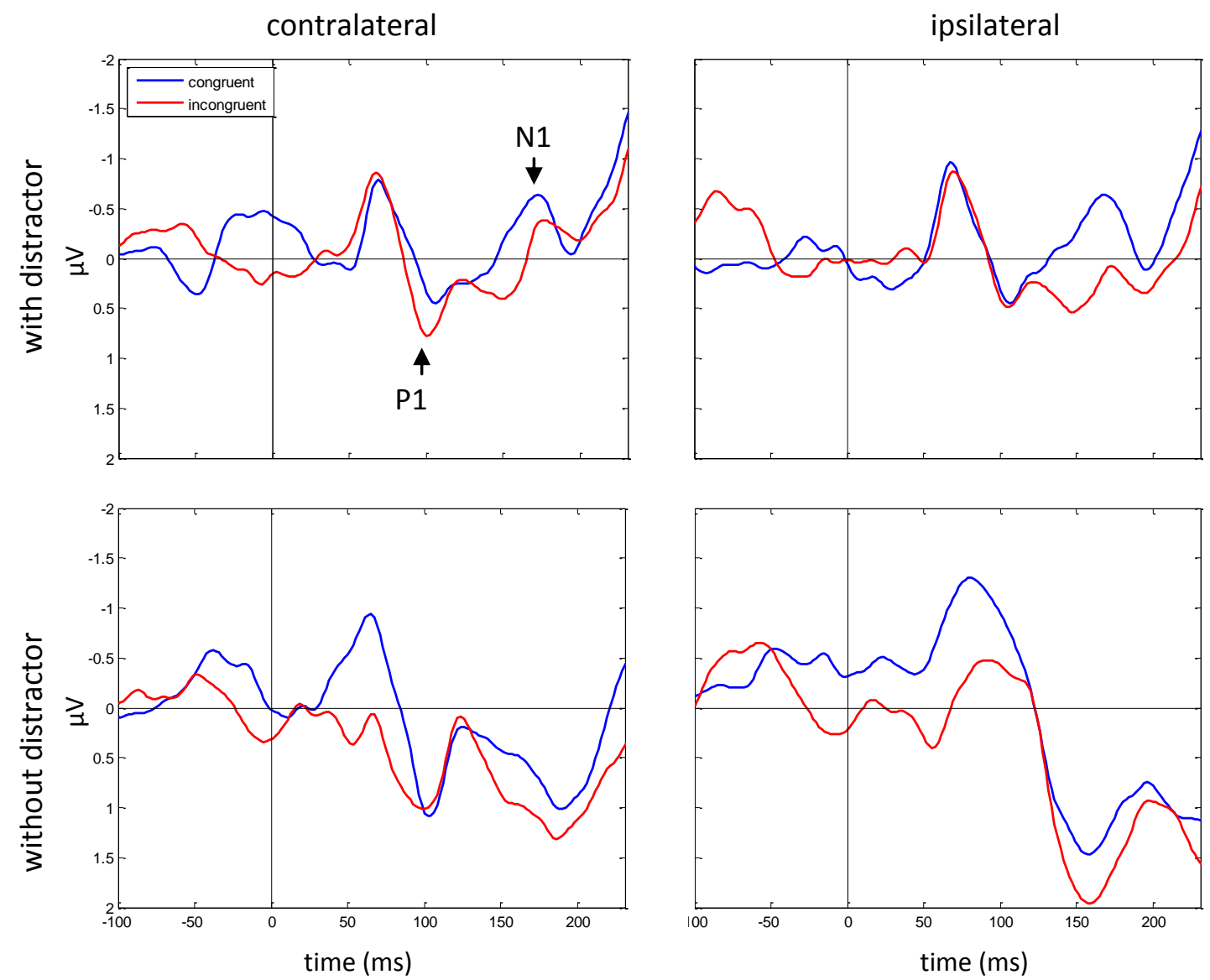

Figure 4.21 Target.-locked ERP differences between short SOA and long SOA trials for congruent and incongruent trials at PO7/PO8. ERPs were collapsed over left- and right-cue conditions and left andright hemispheres to obtain waveforms recorded and contralaterally (left panels) and ipsilaterally (right panels) to the side of the cue. Separated for trials with distractor (top panels), and trials without distractor (bottom panels).

not on trials with left targets. Amplitudes were more negative with than without distractor. An Electrode Side x Cued Side interaction, $F(1,12)=23.1, p<.001, M S e=0.84$, indicated that there was a difference between left and right targets at PO7 but not at PO8. Finally, an Electrode Side x Cued Side x Distractor interaction, $F(1,12)=9.1, p=.011, \mathrm{MSe}=0.8$, indicated that the effect of distractor presence was more pronounced at the electrode ipsilateral to the cued side.

Short SOA - Long SOA 
There were differences between congruent and incongruent trials in potentials during the period in which we expected P1 and N1 elicited by target stimuli, which were probably caused by differing prime and cue symbols. Therefore, we used another approach to assess priming effects on target potentials. Subtracting prime-locked waveforms from the long SOA conditions from those from the short SOA condition should allow us to disentangle differences caused by cues and targets by removing deflections caused by primes and cues. Up until $495 \mathrm{~ms}$ after prime presentation the only difference between long SOA and short SOA conditions is the presentation of the target in the short SOA condition. Thus, differences in potentials until that point in time can be considered effects of target presentation. These difference waves are given in Figure 4.21.

$P 1$. There was no main effect or interaction involving Congruency $(F<2.5, p>.15)$. During the P1 period, there was an Electrode Side x Cued Side interaction, $F(1,12)=13.8, p$ $=.003, M S e=0.73$. Amplitude was increased at the contralateral electrode $(0.4 \mu \mathrm{V})$ compared to the ipsilateral electrode $(-0.04 \mu \mathrm{V})$. There was a significant Electrode Side $\mathrm{x}$ Distractor interaction, $F(1,12)=9.7, p=.009, M S e=0.2$. The distractor effect (present-absent) was negative at PO7 $(-0.52 \mu \mathrm{V})$, but positive at PO8 (0.36). An Electrode Side x Cued Side x Distractor interaction, $F(1,12)=5.3, p=.029, M S e=0.88$, indicated that $\mathrm{P} 1$ was increased in the presence of a distractor at electrodes ipsilateral to the cued side (difference $0.36 \mu \mathrm{V}$ ), but not at contralateral electrodes $(-0.28 \mu \mathrm{V})$.

N1. For the N1 period a main effect of Congruency emerged, $F(1,12)=4.8, p=.048$, $M S e=3$, as amplitude during the N1 period was larger on congruent trials $(0.25 \mu \mathrm{V})$ than on incongruent trials $(0.78 \mu \mathrm{V})$. N1 was more negative for trials with distractor $(-0.09 \mu \mathrm{V})$ than for trials without distractor $(1.13 \mu \mathrm{V}), F(1,12)=14.2, p=.003, M S e=5.4$. An Electrode Side x Cued Side interaction, $F(1,12)=5.1, p=.044, M S e=2$, indicated that N1 amplitude was 
higher at the ipsilateral electrode $(0.74 \mu \mathrm{V})$ than at the contralateral electrode $(0.3 \mu \mathrm{V})$, consistent with the assumption that $\mathrm{N} 1$ was increased at the contralateral side.

\subsubsection{Discussion}

With 164 ms cue-target SOA, target-locked visual potentials showed a similar pattern as in Experiment 9. It is unclear, however, whether these similarities are caused by similar priming effects on target processing or are correlates of effects on cue processing. Visual inspection of ERPs during the baseline time window suggests congruency effects on cue P1 and N1. This might indicate that primes modulate cue processing through repetition priming. Later differences in target-locked potentials between congruent and incongruent trials might still reflect differences in cue processing. Additional priming effects were found during a later time window (280-330 ms). Visual inspection suggests that this was a modulation of a positive going component which was maximal at central parietal electrodes and was increased on incongruent trials. It is not clear what kind of process this modulation might reflect. It seems unlikely that they are related to cue processing because they stand in contrast to Experiment 9, where a negative going component was enhanced on incongruent trials. Rather, this modulation might reflect differences between the letter discrimination and bar discrimination tasks, e.g., a decision about the length of a particular bar, or length comparison between target bar and distractor bar. In fact, most participants reported that the presence of a distractor made the task easier because it allowed them to compare the two bars.

When we computed the short SOA - long SOA difference to exclude shifts caused by primes and cues, we found a modulation in the range of target $\mathrm{N} 1$ at occipital electrodes. However, in this difference waveform, short SOA targets elicited only weak shifts compared to the long SOA targets and there was no accompanying modulation of frontal or central N1 by Congruency. Therefore, this weak effect of Congruency may seem similar to the N1 
priming effect in Experiment 8, but has to be met with caution. Taken together, results with short cue-target SOA provide some evidence that early target processing may be affected by masked primes with the SOA used in Experiment 9.

With $400 \mathrm{~ms}$ SOA, behavioral priming effects surprisingly reversed and responses were faster on incongruent trials than on congruent trials. This reversed priming effect did not seem to be based on differences in early target processing, as P1 and N1 were not affected by primes. Instead, it was accompanied by priming effects on an N2 component which was more pronounced on incongruent trials. Unfortunately, this surprising reversal of priming effects hinders interpretation of results regarding the role of cue-target SOA in cue-priming. Without distractors, the long SOA condition was very similar to the $94 \mathrm{~ms}$ SOA condition in Experiment 8. Therefore, it may seem that the reversal of priming effects in Experiment 10 represents a failure of replication of the priming effect found in Experiment 8. However, there were notable changes from Experiment 8 to Experiment 10 like the use of a more complex prime and cue arrangement and the mixture with short SOA trials and trials with distractor which may explain this reversal.

A similar reversal of cue validity effects has been termed inhibition of return (IOR). IOR was first reported for peripheral cue stimuli (Posner \& Cohen, 1984), but has since been found for central gaze cues as well (Frischen, Smilek, Eastwood \& Tipper, 2007). This indicates that it can also occur with certain central cues. IOR usually occurs with long cuetarget SOAs. Given that SOAs in the long condition of Experiment 10 matched those used in Experiment 8, it seems surprising that IOR would occur in Experiment 8, but not in Experiment 10. In an earlier experiment, we found that spatial cue-priming effects, like other cue-priming effects, decrease with cue-target SOA (Project 1), although we did not find priming effects below zero with 400 ms cue-target SOA. However, in this earlier experiment 
we used a longer prime-cue SOA which could have prevented effects from being inhibited below zero. Reversal of priming effects has been reported in other experiments, but is usually found in experiments that feature a separate mask between prime and cue or target stimulus (e.g., Eimer \& Schlaghecken, 1998; Mattler, 2007). Physiologically, priming effects were found on an $\mathrm{N} 2$ component that was enhanced on incongruent trials. It seems that during this period, a negative going component was enhanced by distractor presence as well as by the presence of incongruent primes. These effects showed some lateralisation but inconsistent and not in the way of an N2pc component that has been found in visual search experiments (Luck and Hillyard, 1995). In Experiment 8 as well as previous experiments (Eimer, 1993; Mangun \& Hillyard, 1991), attention modulated N2 in the opposite direction i.e. N2 was enhanced on congruent and valid trials. Thus, this reversal might correspond to the reversal of behavioral priming effects. However, given that it is not clear what kind of operation is reflected by this $\mathrm{N} 2$ component in the present experiments, additional research is needed to provide insight into this particular reversal of priming effects.

Responses were faster on trials without distractor than on trials with distractor. Thus, it seems that distractors were processed to some degree. Priming effects - behavioral as well as physiological - on the other hand, were unaffected by distractor presence. This suggests that primes affect target processing in a way that is independent of whether a distractor is present in processing or not. Thus, primes might modulate attention at early levels of processing, probably affecting signal quality of the target which is independent of distractors because at later stages, distractors likely play a role in response selection.

Waveforms for trials with distractor were similar to those elicited by targets in Experiment 9. With short cue-target SOA, we found more positive amplitudes on congruent than on incongruent trials during a period which corresponds to the target $\mathrm{P} 1$. This could be 
taken as evidence for P1 modulation by primes. However, target-locked potentials with short cue-target SOA were contaminated by shifts elicited by primes and cue. Thus, this effect could alternatively be explained by differences in cue processing. This view is supported by the fact that no P1 modulation by Congruency is apparent in the short SOA - long SOA difference.

Overall, responses seemed to be slower than in Experiment 8, probably because the presence of distractor stimuli and the introduction of a relatively short cue-target SOA made the task more difficult.

In sum, it seems that the absence of early perceptual effects in Experiment 9 was not due to short cue-target SOA, because N1 modulation did occur with the same cue-target SOA in Experiment 10. The presence of distractor stimuli affected neither behavioral nor physiological priming effects. Thus, it does not seem to be a crucial factor in the magnitude or the locus of priming effects. Instead, we propose that the letter stimuli in Experiment 9 were unsuited to promote attentional selection at early levels because they impose only low amounts of perceptual load.

\subsection{General Discussion of Project 3}

Overall, results show that spatial cue-priming effects are accompanied by a modulation of early visual potentials, as well as later post-perceptual frontal and parietal components. In Experiment 8, weak but consistent behavioral priming effects were associated with a modulation of target $\mathrm{N} 1$ under conditions with very low prime visibility. This was replicated to some degree in the short SOA - long SOA difference in Experiment 10. In Experiment 9, early visual processing of task irrelevant probe stimuli was not affected by primes, even though we found clear behavioral priming effects on discrimination of letter targets. However, 
this does not necessarily mean that early target processing was unaffected by primes. Instead, it might be that there was a lack of priming effects on probe processing because the probes were not task relevant. With short SOAs in Experiments 9 and 10, we found a different pattern of priming effects on target-locked potentials. There was an early modulation of potentials over occipital areas, but instead of an increased N1, we found increased positivity in the period corresponding to the $\mathrm{P} 1$. The design of these experiments makes an attribution of these effects to target processing problematic because of a confoundation with cue processing. Priming effects on later potentials show a more diverse pattern. Modulation of later frontal and parietal components by primes could be interpreted as priming of cue processing and implementation of stimulus-response mapping or task set. Thus, it seems that priming effects in Experiments 9 and 10 are not entirely based on the same processes as in Experiment 8. The critical difference might be found in the presence of a distractor in Experiment 9 and half of the trials of Experiment 10, which introduces the requirement to select between one of two potential target stimuli. Without such a distractor, participants cannot use the cue to decide whether to react to the right or to the left target stimulus, but only to enhance processing of the single target stimulus. Nevertheless, the present results do not exclude the possibility that there were priming effects on early target processing in Experiments 9 and 10. Thus, it might be that in these experiments behavioral priming effects resulted from effects on both early and late selection processes whereas in Experiment 8 primes only acted on early target processing. This could explain why behavioral priming effects were smaller in Experiment 8 than in the other two experiments. In Experiments 9 and 10, several congruency effects appeared to be stronger or only apparent when a specific side was cued. This could mean that cues and primes were mainly used to shift attention to one side, perhaps because participants focused attention on the other side by default. However, it seems more likely that these priming 
effects result from differences in cue processing between square and diamond stimuli, because cue symbols were with cued side.

A somewhat puzzling result was obtained from the long SOA condition in Experiment 10 where priming effects were reversed. At present, we do not have a satisfying explanation for this reversal and future research is needed to clarify its cause. Possible candidates include inhibition of return (Posner \& Cohen, 1984) and the negative compatibility effect (Eimer \& Schlaghecken, 1998).

\section{Mechanisms of priming of spatial attention}

Overall, it seems that there is considerable variability of priming effects in different tasks. In Experiment 8, small congruency effects were apparent in early target processing. In Experiment 9, larger behavioral effects were found along with physiological priming effects on central and parietal sites. However, we found no evidence that primes modulate processing of stimuli at the non-cued location in Experiment 9. This indicates that primes cannot initiate endogenous shifts of attention by themselves. In Experiment 10, priming effects seemed to be based on both early and late visual processing of targets. These results can be explained by assuming that the locus of attentional selection in a given task depends on where conflict occurs (Luck \& Hillyard, 2000). Assuming that participants adapt to use cues in a way that benefits their performance, it seems reasonable to assume that primes activate the same processes as cues. Therefore, priming effects at early levels of processing are found when the task is perceptually difficult (Experiments 8 and 10) but not when the difficulty of the task lies in selecting the correct response. This similarity of prime processing to cue processing suggests that masked symbolic cues can affect the allocation of spatial attention at multiple levels of target processing. However, it is unclear to which extent priming effects in Experiments 9 and 10 are based on perceptual priming of cue processing. This difference 
between the Experiments was also reflected in the modulation of early visual cue related potentials by congruency which was prominent in Experiments 9 and 10, but not in Experiment 8. However, priming effects on later components, that were proposed to reflect cue processing in Experiment 9, were not found in Experiment 10 where the same prime and cue stimuli were used. This suggests either that cue processing differs between the two tasks or that these priming effects are related to target processing.

One serious shortcoming in our experiments is that with short cue-target SOAs electrophysiological effects of target processing cannot be separated from effects of prime and cue processing. Future studies should include conditions without target stimuli which could be used to isolate target induced shifts by subtracting correlates of prime and cue processing.

\subsubsection{Conclusion}

In summary it seems as though the mechanisms of spatial cue-priming effects depend in similar way on task demands as spatial cueing effects. With high perceptual load (bar targets), primes modulate target processing as early as N1, whereas with letter targets primes seem to exert influence at later levels. Priming effects might be larger with letter targets because later processes are more readily affected by primes than selection at early levels.

However, it seems that spatial cue-priming effects are more sensitive to specific task parameters and cue-target SOA than previously thought. Thus, in order to draw conclusions about unconscious processing from cue-priming, careful interpretation of cue-priming effects is required because there can be several possible underlying mechanisms.

\subsection{Summary of Project 3}

In Project 3, electrophysiological correlates of early visual processing were obtained in order to provide evidence that masked priming of spatial attention modulates early target 
processing. Spatial attention has been found to modulate the visual P1 and N1 components of target-locked event related potentials. Consequently, if masked primes affect spatial attention at early levels of processing these potentials should be enhanced on congruent trials compared to incongruent trials. In Experiment 8, we applied spatial cue-priming in a bar discrimination task without distractors. In a similar task, Mangun \& Hillyard (1991) found effects of cue validity on early visual potentials. Compared to the experiments in Project 1, we found surprisingly small priming effects on RT which were significant only with a long prime-cue SOA of $94 \mathrm{~ms}$. These behavioral priming effects were accompanied by a modulation of central and occipital target N1 which was enhanced on congruent compared to incongruent trials. This finding suggests that masked primes in Experiment 8 modulated a perceptual limited capacity discriminatory process. Given that primes and cues in Experiment 8 were perceptually dissimilar, this provides evidence that masked stimuli can affect attention at early levels of target processing.

Experiment 9 was aimed at using electrophysiological measures to investigate attentional effects at the non-cued side. Interpretation of cue-priming effects suffers from the fact that it is not clear whether primes affect attention directly or affect only processing of cue stimuli. This problem might be solved by showing priming effects on stimuli presented on the non-cued side. If primes can directly affect spatial attention, incongruent primes should direct attention to the non-cued side whereas congruent primes direct attention to the cued side. This should lead to enhanced potentials for stimuli on the non-cued side. In order to provide a measure for processing of stimuli on the non-cued side, we used a design with task irrelevant probe stimuli. Spatial attention has been shown to affect visual potentials elicited by task irrelevant stimuli in a visual search task (Luck \& Hillyard, 1995). Therefore, we expected a similar modulation of probe related P1 and N1 components with spatial cue-priming. We used 
a letter discrimination task with distractor and a short cue-target SOA because these conditions produced the largest priming effects in Project 1. Probe stimuli where white square outlines around target locations which were presented before target onset. Probe stimuli were presented on the cued side and on the non-cued side on one third of trials, respectively. On the remaining third of trials, no probe stimulus was presented. These no probe trials were used to isolate probe evoked potentials by calculating difference waveforms for probe and no probe trials. RT in Experiment 9 was affected by prime-cue congruency as well as probe location. As expected we found larger priming effects (about $30 \mathrm{~ms}$ ) than in Experiment 8 and participants responded faster when the probe was presented at the cued location, and slower when it was presented at the opposite location. Priming effects, however, were unaffected by probes. Visual probe-evoked potentials were mostly unaffected by attention as there was no difference between probes presented at the cue location and probes presented at the non-cued location. Probe N1 for cued probes was increased on incongruent compared to congruent trials. Apart from this effect, probe-evoked potentials were unaffected by primes. These findings might be explained by assuming that there was no allocation of spatial attention at the time of probe presentation because probes were presented too shortly after targets. Analysis of target-locked ERPs on no probe trials revealed congruency effects in the period of early visual components. However, interpretation of these effects suffers from an overlap with potentials induced by primes and cues, due to the short cue-target SOA. In addition, later frontal and parietal components were modulated by congruency. Therefore, it is unclear whether priming effects on RT are based on attentional effects of primes at early stages or selection operates at later stages of processing. The finding that primes did affect frontal and parietal potentials might suggest the latter. Comparing Experiments 8 and 9 yields a complex pattern of results. Whereas in Experiment 8 small priming effects with long cue-target SOAs were accompanied by a modulation of target N1, larger priming effects in Experiment 9 were 
found with short cue-target SOAs, but there was no effect on early visual probe related potentials. This is reminiscent of the finding that priming effects on signal detection in Project 2 were found only with longer cue-target SOAs whereas priming effects on letter discrimination were largest with short cue-target SOAs. We speculated that this difference might be due to the presence of a distractor stimulus that introduced conflict in selection processes as opposed to perceptual processes and thereby led to a different focus of attentional processes. It might be that time courses differ for priming effects on selection processes and priming effects on perceptual target processing.

Experiment 10 was conducted to clarify these apparent discrepancies by comparing priming with short cue-target-SOA to priming with long cue-target SOA and also comparing priming without distractor as in Experiment 8 to a condition with distractor similar to Experiment 9. To this end, we varied cue-target SOAs in two steps corresponding to the SOAs used in Experiments 8 and 9, respectively. We employed a bar discrimination task as in Experiment 8. On half of the trials, however, the cued bar was accompanied by a distractor bar at the non-cued side which was short when the target was long and long when the target was short. Combination of SOAs and distractor presence led to four conditions which were presented in random order. Unfortunately, Experiment 10 did not help revealing the important differences between Experiments 8 and 9. Instead the overall picture was made more complex by the finding that there were reversed priming effects with the long $400 \mathrm{~ms}$ SOA, i.e. responses were faster on incongruent trials than on congruent trials. This reversal is surprising especially since the long SOA condition without distractor is very similar to the $94 \mathrm{~ms}$ primecue SOA in from Experiment 8. The reversal of priming effects thus seems to be associated either to changes in prime and cue symbols which were easier to identify in Experiment 10 than in Experiment 8 or to intermixture with trials with distractor or trials with short cue- 
target SOA. The short cue-target SOA and the easier cues might have led to faster shifts of attention on congruent trials than in Experiment 8 which were then followed by inhibition of return.

In addition to these attempts at showing priming effects on early levels of target processing, two other projects were conducted to study the general preconditions of spatial cue-priming. In Project 4 the possibility of cue-priming effects on participants' free choices for one side or the other was examined. In Project 5, we tried to study cue-priming effects under conditions in which the cue did not always predict the correct target location. 


\section{Project 4 - Free choice cue-priming}

In order to investigate whether primes affect decisions on a central level of processing, we employed a free choice task that allowed us to directly measure the effects of primes on participants' decisions to shift attention to either side. Free choice priming has been found to produce reliable effects on the choice between two competing motor responses (e.g., Kiesel et al., 2006; Mattler \& Palmer, 2012; Schlaghecken \& Eimer, 2004). Typically, in free choice priming trials with free choice targets, allowing participants to choose freely among two response alternatives, are presented among forced choice trials with imperative targets, specifying which response has to be executed. The presentation of forced-choice trials is necessary to provide the context for subliminal primes to be effective (Klapp \& Haas, 2005).

\subsection{Experiment 11}

In Experiment 11, we combined free choice priming and cue-priming by introducing trials with free choice cues. The task was similar to the previous experiments, and on two thirds of trials participants had to indicate the symbol signalized by a forced choice cue. On the remaining trials, a free choice cue allowed them to indicate either of the target symbols (left or right). Note, that this paradigm still involves presenting two stimuli - prime and cue before the target in each trial instead of presenting only one cue stimulus like Reuss, Kiesel et al. (2011). However, since the free choice cue provides no information about the following target, it should not affect participants' decisions or the allocation of attention. Furthermore, this paradigm avoids perceptual priming effects because there are no separate informative cue stimuli (Reuss, Kiesel et al., 2011). If primes can influence the decision for a side in a spatial cueing task in a similar manner as the decision for a motor response in response priming, free 
choices should be affected accordingly. This would provide strong evidence that spatial cuepriming effects extend to central stages of processing.

\subsubsection{Method}

Participants. 16 new Participants completed 4 sessions of Experiment 11. Four additional participants were excluded because they either chose the same response on more than $95 \%$ of free choice trials, failed to attend to the cues and responded randomly to the targets, or problems with eye gaze detection.

Task. On forced choice trials, participants had to indicate the symbol (A,B,1 or 2) on the cued side as fast as possible using the F,V,K and M key of the keyboard. Stickers on the keys showed which key coreesponded to which symbol. On trials with a free choice signal, participants were to choose one side, shift their attention to that side, and then indicate the target symbol on that side. We used four different possible targets instead of two to force participants to attend to the targets on free choice trials. If there were only two possible targets and responses, participants could simply respond either way once they see the free choice signal without waiting for the target stimulus.

Design and Procedure. Participants completed 2 sessions with four blocks of 216 trials each. Each possible combination of 2 primes (left, right), 3 cues (left, right, free choice), 3 SOAs (20, 60, $100 \mathrm{~ms}$ ), and 12 targets (all possible combinations of the 4 target symbols) was presented once within each block. After every 36 trials, there was a break that was ended by a key press. In total, this yielded 192 trials in each condition (congruent, incongruent, free choice; 3 SOAs). 


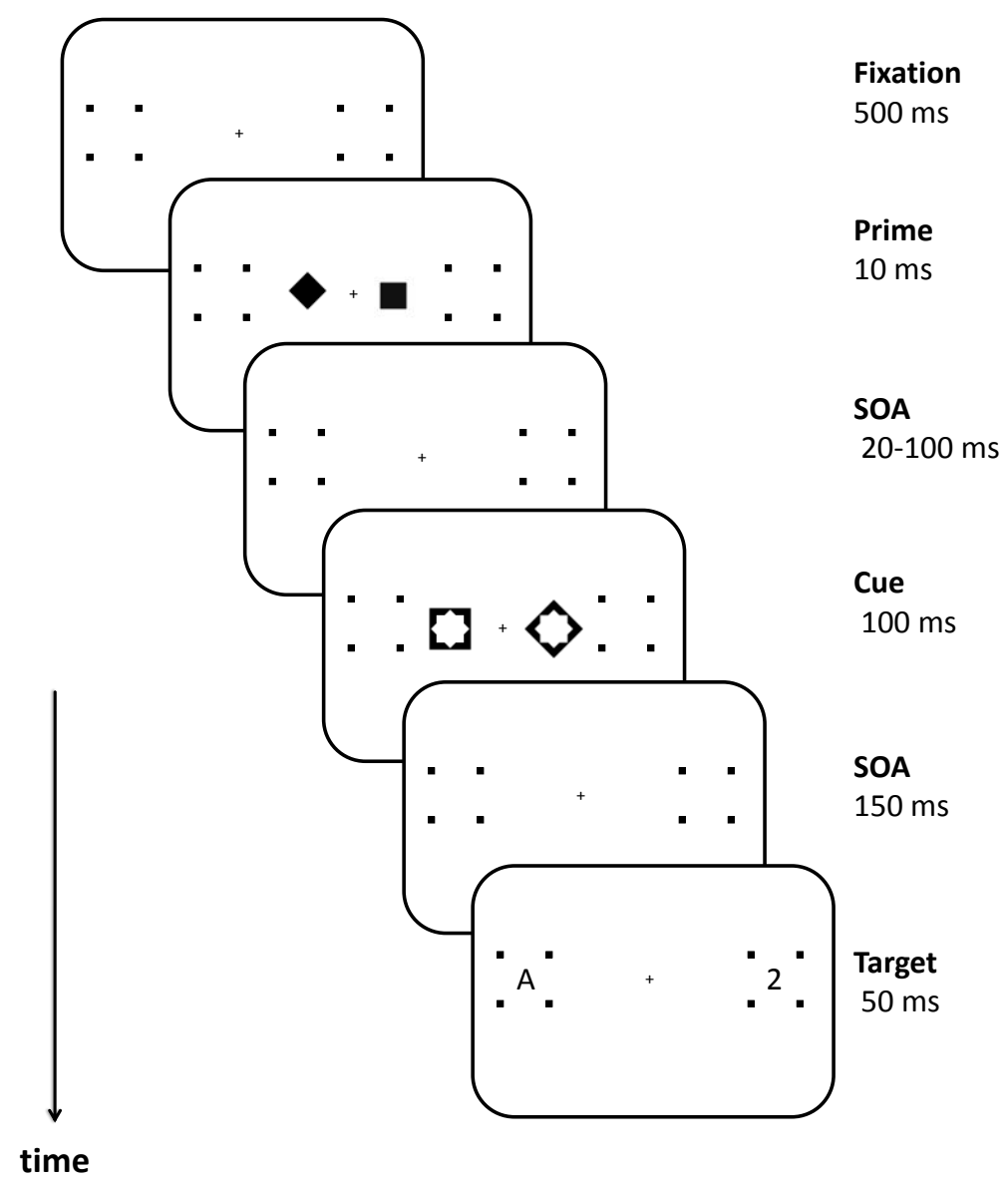

Figure 5.1 Sequence of stimulus events in a trial of Experiment 11.

Stimuli. Figure 5.1 gives an example of the stimulation in one trial of 11. Stimuli were presented using the same setup as before with a vertical refresh rate of $100 \mathrm{~Hz}$. Primes were presented for $10 \mathrm{~ms}$, cues for $100 \mathrm{~ms}$, and prime-cue-SOA varied in three steps $(20,60$, and $100 \mathrm{~ms}$ ). Targets were presented for $50 \mathrm{~ms}$ and participants had $2000 \mathrm{~ms}$ from target offset to respond. Inter-trial-intervals varied quasi-exponentially from 700 to $1750 \mathrm{~ms}$, with shorter intervals being more frequent. Primes and cues always consisted of two symbols, either square or diamond shaped. Forced choice cues were spatially correspondent to target locations, as the position of the diamond indicated the relevant side. The free choice signal consisted of two squares. This configuration has produced reliable free choice priming effects in a motor task (Mattler \& Palmer, 2012). Primes extended $1.9^{\circ}$, cues $1.1^{\circ}$ (edge to edge), 
distance between primes and cues was $4^{\circ}$ from centre to centre. Target symbols subtended $1.3^{\circ} \times 1.1^{\circ}$ visual angle. They were located $8^{\circ}$ visual angle left and right to the centre of the screen.

Apparatus. We used the same setup as in the previous projects. Due to a technical error, eye-movement data were lost for 7 participants. Therefore, we did not exclude trials with eye movements from the main analyses. Instead, we performed separate analyses to see whether excluding trials with eye movements would affect the results for the remaining 9 participants.

Analyses. RTs and error rates for congruent and incongruent trials were computed and analyzed as before. On free choice trials, three types of responses were possible. Participants could either choose the response key associated with the target on the primed side (primedtarget response), choose the response associated with the target on the other side (non-primedtarget response, or erroneously press one of the other two keys (free choice error). We computed mean RTs for primed-target and non-primed-target responses, respectively. Free choice response bias was computed as the proportion of primed-target responses among all correct free choice responses (primed-target + non-primed target responses). All dependent measures were analyzed using repeated measures ANOVAs. We used a signal detection analysis to assess the bias in free choice responses caused by primes. To this end, we defined a left prime as the signal and the decision for either target as the response. Thus, when participants chose the left target after they were presented with a left prime it was considered a hit. Accordingly, when participants chose the left letter after a right prime had been presented, it was considered a false alarm. From this, we obtained free choice d' as a measure of priming effects on free choice decisions. Free choice errors were excluded from this analysis. 


\subsubsection{Results}

Figure 5.2 shows results in Experiment 11. Forced choice responses were faster on congruent $(650 \mathrm{~ms})$ than on incongruent trials $(664 \mathrm{~ms}), F(1,15)=16.4, M S e=312, p=.001$ (Figure 5.2B). Forced choice RT was also affected by SOA, $F(2,30)=5.5, M S e=257, p=$ .015. There was an interaction between Congruency and SOA, $F(2,30)=10.8, M S e=226, p<$ . 001. Post-hoc t-tests comparing RT on congruent to RT on incongruent trials for the three SOAs showed a significant effect only with $100 \mathrm{~ms}$ SOA $(35 \mathrm{~ms}, t(15)=5.8, p<.001)$.

Free choice RTs (Figure 5.2D) were not different when Participants chose the primed symbol (716 ms) than when they chose the non-primed symbol (723 ms), $F(1,15)=1.5, M S e$ $=977, p=.246$, but were affected by SOA, $F(2,30)=5, M S e=290, p=.014$. There was no significant interaction of the two factors, $F(2,30)=1.9, M S e=341, p=.166$.

Participants chose the primed symbol (54.8\%) more often than the non-primed symbol (45.2\%). Mean free choice d' (0.27), was significantly above zero, $t(15)=6.9, p<.001$ (Figure 5.2C). This effect increased with SOA, as indicated by a main effect of SOA on free choice d', $F(2,30)=6.9, M S e=0.06, p=.005$. However, free choice d' was significantly larger than zero for all SOAs, $t(15)>3.6, p<.003$, in all cases.

Response errors (Figure 5.2A) were more frequent on incongruent $(6.6 \%)$ than on congruent trials $(5.6 \%), F(1,15)=7, M S e=0.005, p=.019$. We also found a significant Congruency x SOA interaction, $F(2,30)=4.2, M S e=0.007, p=.031$. t-tests indicated that a significant priming effect on accuracy was only present with $100 \mathrm{~ms} \mathrm{SOA}, t(15)=2.8, p=$ $.012)$. 
A

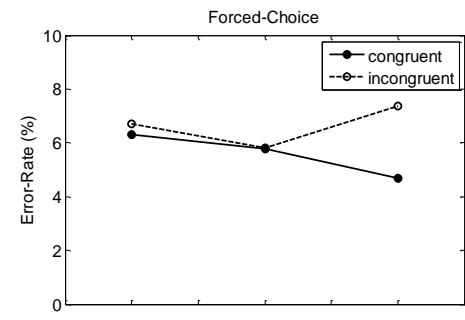

B

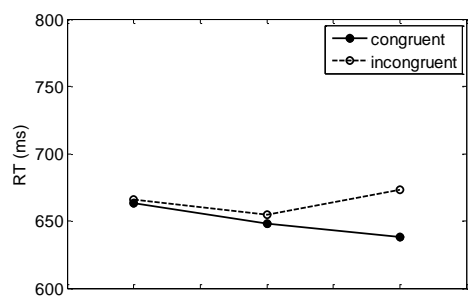

$\mathrm{C}$

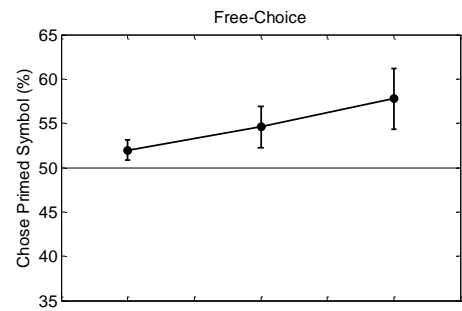

$\mathrm{D}$

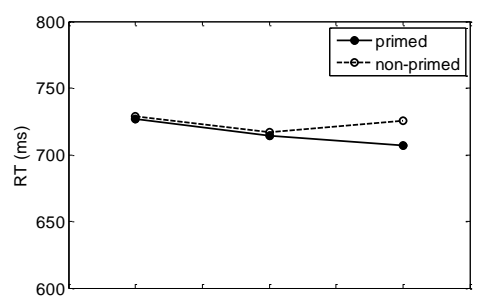

E

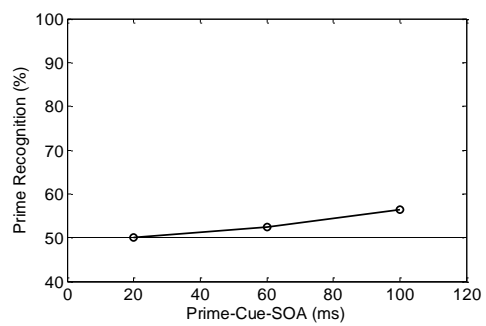

Figure 5.2 Results in Experiment 11. (A) Rate of response errors on congruent and incongruent forcedchoice trials as a function of prime-cue SOA. (B) RT on congruent and incongruent forced-choice trials as a function of prime-cue SOA. (C) Percentage of free choice trials on which participants chose the primed response. Error bars show 95\% confidence intervals. (D) RT on free choice trials separately for trials on which the response was consistent with the primed symbol and trials on which the response was consistent with the non-primed symbol (E) Percentage of correct responses in the prime recognition session. 
Eye-movements were analyzed for a subgroup of 9 participants. As in the previous experiments, eye-movement errors increased with $\mathrm{SOA}, F(2,16)=16.1, M S e=0.043, p=$ $.003(17.9 \%, 22.6 \%$ and $30.7 \%$ with $20 \mathrm{~ms}, 60 \mathrm{~ms}$, and $100 \mathrm{~ms}$ SOA, respectively), but were unaffected by the type of trial (congruent, incongruent or free choice), $F(2,16)=1.8$, $M S e=0.003, p=.2$. When we excluded trials using the same criteria as before, a similar result pattern emerged. Free choice d' was significantly larger than zero with all SOAs (d' > $0.17, t(8)>2.9, p<.002$ in all cases), but was not significantly affected by $\operatorname{SOA}, F(2,16)=$ 1.1, $M S e=0.049, p=.33$. Forced-choice RT was affected by Congruency, $F(1,8)=13.4$, $M S e=169, p=.006$, and SOA, $F(2,16)=4, M S e=248, p=.04$. The Congruency $\mathrm{x}$ SOA interaction emerged here as well, $F(2,16)=5.8, M S e=304, p=.012$. t-tests evaluating the effect of removing trials with eye-movements in the 9 participants where this was possible revealed no effects on either forced-choice RT or free choice d', $t(8)<1.1, p>.3$, in all cases.

Prime recognition performance (Figure 5.2E) improved with increasing SOA, $F(2,30)$ $=14.8, M S e=0.13, p<.001$. t-tests revealed that recognition performance was significantly better than chance with $100 \mathrm{~ms}$ SOA, $t(15)=3.6, p=.002$, but not with $60 \mathrm{~ms}, t(15)=1.6, p=$ .12 , nor with $20 \mathrm{~ms}$ SOA, $t(15)=0.1, p=.91$.

\subsubsection{Discussion}

Primes affected participants' spatial choices on free choice as well as on forcedchoice trials. On free choice trials, this effect emerged even with short SOAs, whereas on forced choice trials, it was only apparent with $100 \mathrm{~ms}$ SOA. This finding is not surprising, considering that on free choice trials, no potentially competing cue stimulus is presented. This allows for smaller influences to bias the decision. In addition, we replicated the effects of prime-cue congruency on forced choice RT which was, however, of smaller magnitude than in the previous experiments. With $20 \mathrm{~ms}$ SOA, a significant bias towards choosing the primed 
target was found, even though primes could not be discriminated better than chance. Thus, it seems that masked primes can affect the selection of targets based on spatial location even when participants are unaware of their presence. RT on free choice trials was not affected by whether participants chose the symbol on the primed or the non-primed side. This could suggest that primes did not affect early target processing which would have led to faster processing on the primed side. Instead, it seems that primes bias selection at later levels of processing.

Results show that masked primes can affect spatial attention even when participants are free to choose between two sides. This suggests that cue-priming effects extend beyond priming of cue processing, because no relevant cue was presented on free choice trials. Moreover, this free choice cue-priming effect increases with prime-cue SOA in a similar way as free choice priming of motor responses. This could mean that the mechanisms by which masked primes affect the selection of motor responses or the selection of target symbols as in this experiment are similar. Perhaps, free choice priming of spatial attention can be modelled in a similar way as the decision between competing motor responses (Mattler \& Palmer, 2012). In that study, we found that spatial compatibility is an important factor in free choice priming. As spatial compatibility also plays an important role in spatial cueing (e.g., Lambert, Roser, Wells \& Heffer, 2006), we cannot be sure whether free choice priming of spatial attention is possible with symmetrical symbols like those used in other experiments. The overall larger amount of eye-movement errors in this experiment compared to the experiments in Project 1 is likely due to the non-central presentation of primes and cues which might have made it more difficult to keep fixation on the centre of the screen. 


\subsection{Experiment 12}

In an earlier experiment on free choice response priming we found that free choice priming effects depended on spatial compatibility of responses and prime and target stimuli. With asymmetrical stimuli, considerable free choice and forced choice priming effects were found, whereas symmetrical stimuli led to a similar amount of forced choice priming,, but only marginal free choice priming. In spatial cueing with central cues, spatial correspondence seems to play an important role as well (Lambert \& Duddy, 2002; Shin et al. 2011). The role of spatial correspondence bears potential relevance to the question about the underlying mechanisms of spatial cue-priming. Cueing effects of certain types of symmetrical central cues like arrows and eye gaze have been linked to automatic involuntary processing. Thus, it might be that in Experiment 11 priming effects were based on such involuntary mechanisms. Forced-choice spatial cue-priming is possible with symmetrical stimuli, but might be caused by priming of cue processing, perceptual or otherwise. Free choice priming effects with symmetrical stimuli would provide evidence that prime processing in spatial cue-priming extends beyond priming of cue processing and actually affects target selection independently of cue stimuli. Therefore, in Experiment 12 we tried to replicate Experiment 11 with symmetrical prime and cue stimuli.

\subsubsection{Method}

Participants. 11 new Participants completed 4 sessions of Experiment 12. Two additional participants were excluded because of problems with eye gaze detection or data loss.

Task. The task was the same as in Experiment 11, with the exception that different cue and prime symbols were used. In Experiment 12, a single square was associated to the left 
side, and a single diamond was associated to the right side. Free choice trials were signalled by an octagon.

Design and procedure.

Design and procedure were the same as in Experiment 11.

Stimuli. Stimuli were the same as in Experiment 11 with the following differences. Instead of two symbols, only one symbol was presented at a time and the shape instead of the position mattered. Primes and cues were the same size as in Experiment 1 and were presented at fixation.

Apparatus. We used the same setup as in Experiment 11.

Analyses. Data were analyzed the same way as in Experiment 11, with the difference that no data loss occurred, and eye movements could be analyzed for all participants.

\subsubsection{Results}

Results in Experiment 12 are given in Figure 5.3. Forced-choice responses were faster on congruent (806 ms) than on incongruent trials $(822 \mathrm{~ms}), F(1,10)=37.7, M S e=111$, $p<.001$ (Figure 5.3B). Forced choice RT was also affected by SOA, $F(2,20)=12.9, M S e=$ $371, p<.001$. There was an interaction of Congruency and SOA, $F(2,20)=3.9, M S e=575, p$ <. 037. Post-hoc t-tests comparing RT on congruent to RT on incongruent trials for the three SOAs showed a significant effect only with $100 \mathrm{~ms} \mathrm{SOA}(39 \mathrm{~ms}, t(10)=4.2, p=.002)$. 
A

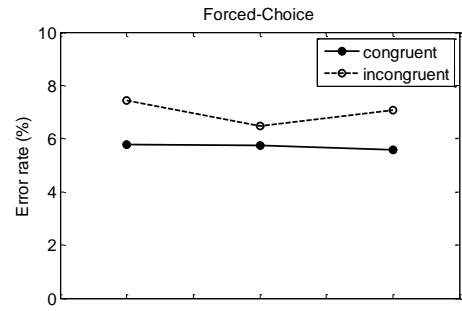

$\mathrm{B}$

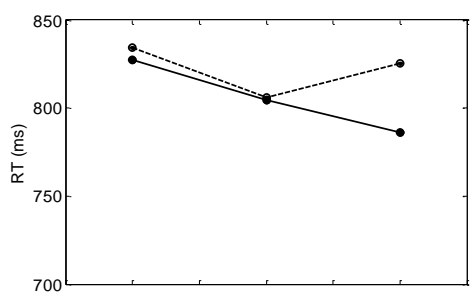

C

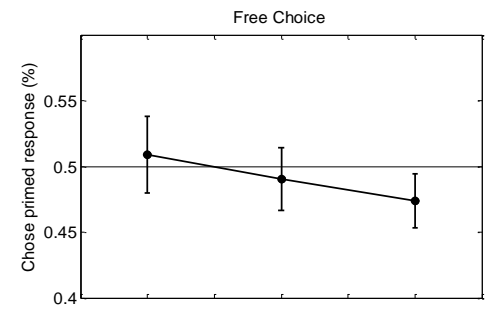

D

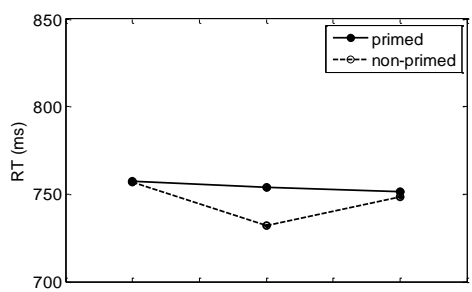

$\mathrm{E}$

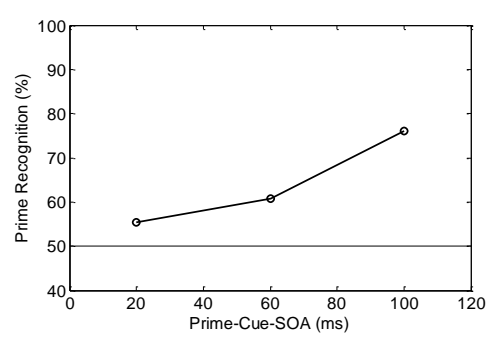

Figure 5.3 Results in Experiment 12. (A) Rate of response errors on congruent and incongruent forced-choice trials as a function of prime-cue SOA. (B) RT on congruent and incongruent forced-choice trials as a function of prime-cue SOA. (C) Percentage of free choice trials on which participants chose the primed response. Error bars show 95\% confidence intervals. (D) RT on free choice trials separately for trials on which the response was consistent with the primed symbol and trials on which the response was consistent with the non-primed symbol (E) Percentage of correct responses in the prime recognition session. 
On free choice trials participants responded slower when they chose the primed symbol (754 ms) than when they chose the non-primed symbol (745 ms), $F(1,10)=5.6, M S e$ $=209, p=.039$, but RT was not affected by SOA, $F(2,20)=2.1, M S e=531, p=.152$ (Figure 5.2D). There was no significant interaction of the two factors, $F(2,20)=1.9, M S e=381, p=$ .169 .

Participants did not choose the symbol on the side corresponding to the primed symbol (49.1\%) more often than the non-primed symbol (51.9\%), mean free choice d' was -0.04 , which is not different from zero, $t(10)=-1.5, p<.168$ (Figure 5.2C). There was no main effect of SOA on free choice d', $F(2,20)=2.2, M S e=0.04, p=.014$. Surprisingly, free choice d' was significantly smaller than zero with $100 \mathrm{~ms}$ SOA, $t(10)>2.7, p<.021$. Response errors (Figure 5.2A) were more frequent on incongruent (7\%) than on congruent trials $(5.7 \%), F(1,10)=7.1, M S e=0.006, p=.023$. There was no significant Congruency $\mathrm{x}$ SOA interaction, $F<2, p>.2$.

Eye-movement errors increased with SOA, $F(2,20)=15.0, M S e=0.01, p<.001(6.2$ $\%, 7.5 \%$ and $9.5 \%$ with $20 \mathrm{~ms}, 60 \mathrm{~ms}$, and $100 \mathrm{~ms}$ SOA, respectively), but were unaffected by the type of trial (congruent, incongruent or free choice), $F<2, p>.3$.

Prime recognition performance (Figure 5.2E) improved with increasing SOA, $F(2,20)$ $=15.0, M S e=0.43, p<.001$. $\mathrm{t}$-tests revealed that recognition performance was significantly better than chance with all SOAs, $t(10)>2, p<.05$, in all cases.

\subsubsection{Discussion}

In Experiment 12 there were significant priming effects on RT and error rates on forcedchoice trials. However, free choice responses were not affected by primes in the predicted manner, as prime congruent responses were neither faster nor more frequent than prime- 
incongruent responses. This finding is consistent with the assumption that spatial cue-priming depends to a large part on (perceptual) priming of cue processing, which is only possible on forced choice trials. In addition, it is similar to what was found for priming of motor responses (Mattler \& Palmer, 2012), where free choice priming effects are also much larger with spatially compatible stimuli, although, in contrast to the present study, weak free choice priming effects were found with symmetrical stimuli. Surprisingly, Experiment 12 provides some evidence for reversed priming effects on free choice trials, as prime-incongruent responses were faster than prime-congruent responses and, with $100 \mathrm{~ms}$ SOA, more frequent. This might be due to perceptual interactions between primes and the free choice signal which may have led to prime-opposing percepts, e.g., by virtue of object updating (Lleras \& Enns, 2004).

\subsection{General Discussion}

From the present results, it seems that free choice cue-priming of spatial attention is possible, but only with spatially compatible primes and cues. This suggests that cue-priming effects, at least with this type of stimuli, do not depend on priming effects on cue processing. With symmetrical stimuli, however, no free choice priming was found. A critical role of spatial compatibility in spatial cueing has been proposed by Lambert and colleagues (Lambert \& Duddy, 2002; Lambert Roser, Wells \& Heffer, 2006). They assumed that spatial correspondence facilitates translation of cues to spatial representations. Thus, an important difference between the spatially compatible primes used in Experiment 11 and the symmetrical primes used in Experiment 12 might be their ability to translate to spatial representations, which are crucial for free choice priming effects. On forced choice trials, even symmetrical primes can affect RT. Experiment 3 shows that symmetrical symbolic stimuli exert effects by perceptual priming on the one hand, but also have access to post- 
perceptual processing. This suggests that access of primes to post-perceptual levels of processing is not sufficient to enable primes to bias free choices. Speculatively, in order to bias choices for one side, primes have to provide a large enough impact to trigger attentional orienting by themselves, without the help of visible cue stimuli. The necessary levels of impact may only be attainable by spatially compatible primes. Thus, although results point to a crucial role of spatial compatibility in enabling cue-priming effects on decisional levels, taken together with other results (Experiment 1), it seems that access to post-perceptual levels of processing is only facilitated rather than exclusively enabled by spatial compatibility.

With regard to the level of target processing affected by primes in the free choice task, it seems likely that primes affect selection at relatively late stages, instead of affecting perceptual target processing. Although participants were instructed to decide for one side before target presentation, it is unlikely that signal quality at early stages was affected, due to low perceptual load and short cue-target SOA.

In conclusion, transfer of cue-priming to a free choice task seems to be a useful method to localize the source of priming effects, as it precludes priming effects on cue processing. It remains an interesting question whether free choice spatial cue-priming can also affect early levels of target processing.

\subsection{Summary of Project 4}

Project 4 examined the possibility of free choice priming of spatial attention. Priming effects on free choices have been reported for choices between motor responses (Eimer \& Schlaghecken) as well as choices between two task sets (Reuss, Kiesel et al., 2011) and were taken as evidence for direct effects of primes on behavior as opposed to priming of cue processing. To this end, we employed a symbol discrimination task similar to the task used in 
Project 1 and presented a free choice cue on one third of trials. This cue allowed participants to choose and respond freely to one of the presented symbols. We expected that participants would select the symbol on the side associated to the prime symbol more often than chance would suggest. This was the case in Experiment 11, in which spatially compatible displays of two symbols were used as cues and primes, but not in Experiment 12, in which primes consisted of a single square or diamond. Results suggest that masked primes can directly access the allocation of spatial attention, i.e. the selection of one symbol over the other, in this task. However, this access may be restricted to spatially compatible primes, a pattern that resembles free choice priming of motor responses (Mattler \& Palmer, 2012) and points to a crucial role of specific stimulus attributes in spatial cueing (Lambert \& Duddy, 2002). 


\section{Project 5 - The role of cue validity in spatial cue-priming}

The experimental paradigm used in Project 1 differs from typical spatial cueing experiments (e.g., Posner 1980). In our experiments, cues were always valid and had to be used to select a target from a display of two competing stimuli. In contrast, spatial cueing studies typically feature trials with invalid cues on which the target is presented at non-cued locations. Therefore unambiguous targets have to be used. Thus, it is unclear to which degree the same attentional processes are measured in spatial cue-priming as in spatial cueing. This issue was addressed in Project 5.

\subsection{Experiment 13}

In order to clarify this issue, we conducted an experiment combining spatial cuepriming with targets and cue validity conditions more similar to those usually found in spatial cueing. If spatial cue-priming affects the same attentional processes as visible cue stimuli in spatial cueing, priming effects should transfer to this paradigm. On validly cued trials, this should lead to improved performance on congruent trials compared to incongruent trials. On invalidly cued trials, however, the priming effect might reverse, with congruent primes potentially supporting the effect of the detrimental invalid cue and incongruent primes counteracting the effect of the cue.

\subsubsection{Method}

Participants. 13 participants (6 women, age 18-30, $\mathrm{M}=22.8$ ) completed 3 sessions of the experiment in exchange for $21 €$ or course credit. 4 additional participants were excluded from analyses because of problems with gaze detection or failure to comply with task instructions. 
Task. Participants had to indicate whether a target letter was an A or B using the "+" key and the "Enter" key on the number pad of the keyboard. Target letters were presented at one of two possible locations, either to the left or to the right of fixation, and were accompanied by a distractor letter $(\mathrm{Z})$ at the other location. Target location was predicted by cues with $80 \%$ validity. A square cue signalled a target on the left side, a diamond cue signalled a target on the right side.

Design and Procedure. Participants completed one practice session and 2 proper sessions with five blocks of 160 trials each. Each session started with a short training phase with 200 ms cue-target-SOA In each block, one of the five SOAs $(100,200,300,400,500$ ms) was used. The order of blocks was randomised for each session. Each possible

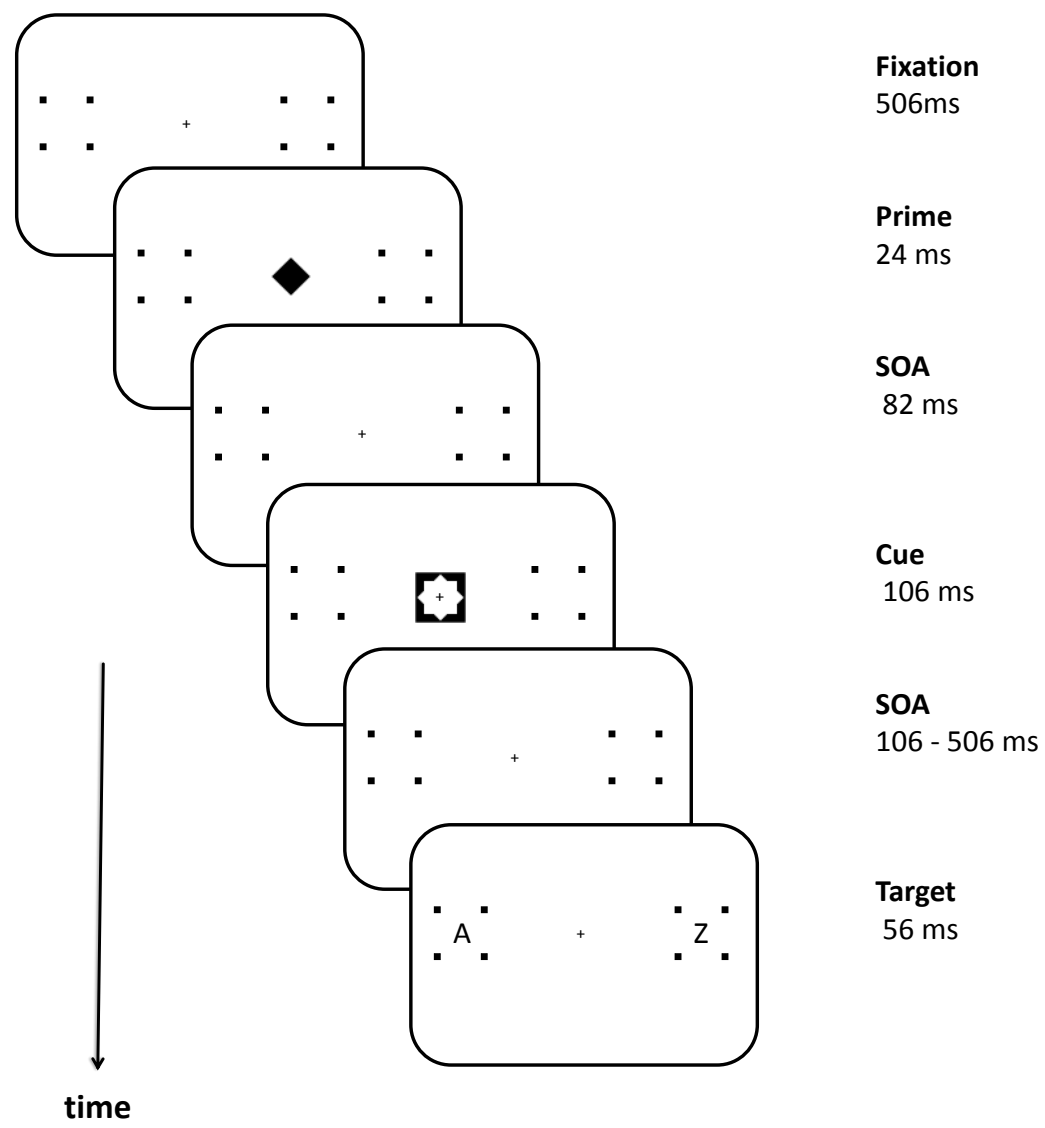

Figure 6.1 Sequence of events in a trial of Experiment 13. 
combination of 2 primes (left, right), 2 cues (left, right) and 2 targets (A, B) was presented 20 times within each block, 16 times with the target letter on the cued side and 4 times with the target letter on the non-cued side. After every 40 trials, there was a break that was ended by a key press. In total, this yielded 128 valid trials and 32 invalid trials in each condition (congruent, incongruent, 5 SOAs, pooling across primes, masks, and targets).

Stimuli. Figure 6.1 gives an example of the stimulation in one trial of Experiment 13. Stimuli were presented using the same setup as in Project 1 with a vertical refresh rate of 85 Hz. Primes were presented for $24 \mathrm{~ms}$, cues for $106 \mathrm{~ms}$ and cue-target-SOA varied in 5 steps (106, 200, 306, 400 and $506 \mathrm{~ms})$. Prime-cue SOA was held constant at $82 \mathrm{~ms}$. Targets were presented for $56 \mathrm{~ms}$ and participants had $2000 \mathrm{~ms}$ from target offset to respond. Inter-trialintervals varied quasi-exponentially from 1000 to $2250 \mathrm{~ms}$ with shorter intervals being more frequent. Primes extended $1.7^{\circ}$, cues $2.7^{\circ}$ (edge to edge). Target letters subtended $1.3^{\circ} \times 1.1^{\circ}$ visual angle and were located $8^{\circ}$ visual angle left and right to the centre of the screen.

Apparatus. We used the same setup as in Project 1.

Analyses. Trials on which eye movements occurred were excluded from analyses using the same method as in Project 1. RTs for congruent and incongruent trials were computed from correct responses for each participant in each condition. Rates of response errors and eye-movement errors were computed for each participant in each condition and arc-sine transformed. All variables were analyzed using $2 \times 2 \times 5$ repeated-measures ANOVAs with factors Congruency, Cue Validity, and SOA.

\subsubsection{Results}

$R T$. There was a main effect of Cue Validity on RT, $F(1,12)=13.3, M S e=6412, p=$ .003 (Figure 6.2A-B), as responses were faster on valid trials (486 ms) than on invalid trials 
(523 ms). The validity effect, measured as the difference between invalid and valid trials increased with cue-target SOA (from $22 \mathrm{~ms}$ to $49 \mathrm{~ms}$ ) as indicated by a Cue Validity x SOA interaction, $F(4,48)=4, M S e=439, p=.014$. There was also a main effect of SOA, $F(4,48)$ $=8.2, \mathrm{MSe}=1471, p<.001$. Numerically, RT was largest with $106 \mathrm{~ms}$ SOA (527 ms), and decreased with SOA until $306 \mathrm{~ms}$ (489 ms) then increased again until $506 \mathrm{~ms}$ (510 ms). Ttests comparing RT on valid trials and invalid trials for single SOAs revealed significant cueing effects with each SOA, $t(12)>2.5, p<.021$, in all cases (uncorrected). Surprisingly, there was no effect of prime-cue congruency, $F<0.01, p>.9$, nor was there a Congruency $\mathrm{x}$ Cue Validity interaction, $F<1, p>.7$.

Response Errors. There was a main effect of Cue Validity on response error rates, $F(1$, 12) $=5.5, M S e=0.0007, p=.038$ (Figure 6.2C-D), indicating that more errors were committed on valid trials $(3.8 \%)$ than on invalid trials $(3 \%)$. All other main effects and interactions were not significant, $F<2.5, p>.05$.

Eye Movement Errors. Rate of eye movement errors increased with increasing SOA $F(4,48)=42.7, M S e=0.1, p<.001$. All other main effects and interactions were not significant, $F<2, p>.1$

\subsubsection{Discussion}

In this paradigm, primes did not have any effects on RT. Thus, it seems that attention was not affected by masked stimuli. In contrast, validity of visible cues had the expected effect on RT which increased with cue-target SOA. How can this absence of priming effects, which is surprising considering that we used similar prime-cue and cue-target SOAs as in Project 1, be explained? The task we used here was more similar to typical spatial cueing experiments as we used unambiguous targets. This potentially enables participants to respond 
without using the cues in any way. Such a strategy was not possible in the earlier letter discrimination experiments, where targets were always accompanied by a relevant distractor and the cue had to be used to find the correct response. This also applies to some extent to the signal detection experiments in Project 2 where perceptual similarity was crucial for priming effects because cues had to be used to dissolve spatial uncertainty about the relevant location, which was introduced by the presence of masks at the non-cued location. Although this aspect of the present experiment probably lowered participants' motivation to attend to the cue stimuli, the presence of effects of cue validity suggests that they nevertheless used the cues to shift attention. It might still be that in order for primes to be effective, participants have to be more motivated or pay more attention to cues than it was the case here. Alternatively, it might be that priming effects crucially depend on cue processing being needed to respond. If primes are effective mainly by modulating cue processing, priming effects will be found when RT is in large part determined by the time it takes to process the cue. Results in Project 1 show that priming effects depend on perceptual similarity of prime and cue. It seems likely that this is the case because primes can perceptually prime attributes of cue symbols. However, perceptual priming of cue processing should also lead to priming effects in this experiment, because the time it takes to process the cue is an important factor for the magnitude of cueing effects. Thus, assuming that primes received enough attention to potentially have effects, the complete absence of priming effects seems to suggest that cue-priming (even perceptual priming of cue processing) is only possible under certain circumstances, i.e. when cues are always valid.

\subsection{Experiment 14}

Results from Experiment 13 are in contrast to results from Project 1 where reliable priming effects were found in a similar letter discrimination task. On the one hand, this may 
be explained by a lack of cue validity in Experiment 13 . On the other hand, the absence of relevant distractor stimuli might have enabled participants to use a different strategy which made them less susceptible to primes. Specifically, without relevant distractors, cue stimuli become less important as they are not crucial to select the correct target and thus the correct response. In Experiment 14 we aimed to clarify the role of distractors' task relevance by directly comparing trials with relevant distractor letters and trials with task-irrelevant distractors with perfectly valid cues. In Experiment 14 cue-target SOA was varied from 100 to $400 \mathrm{~ms}$.

If processing differs between trials with distractor and trials without distractor one
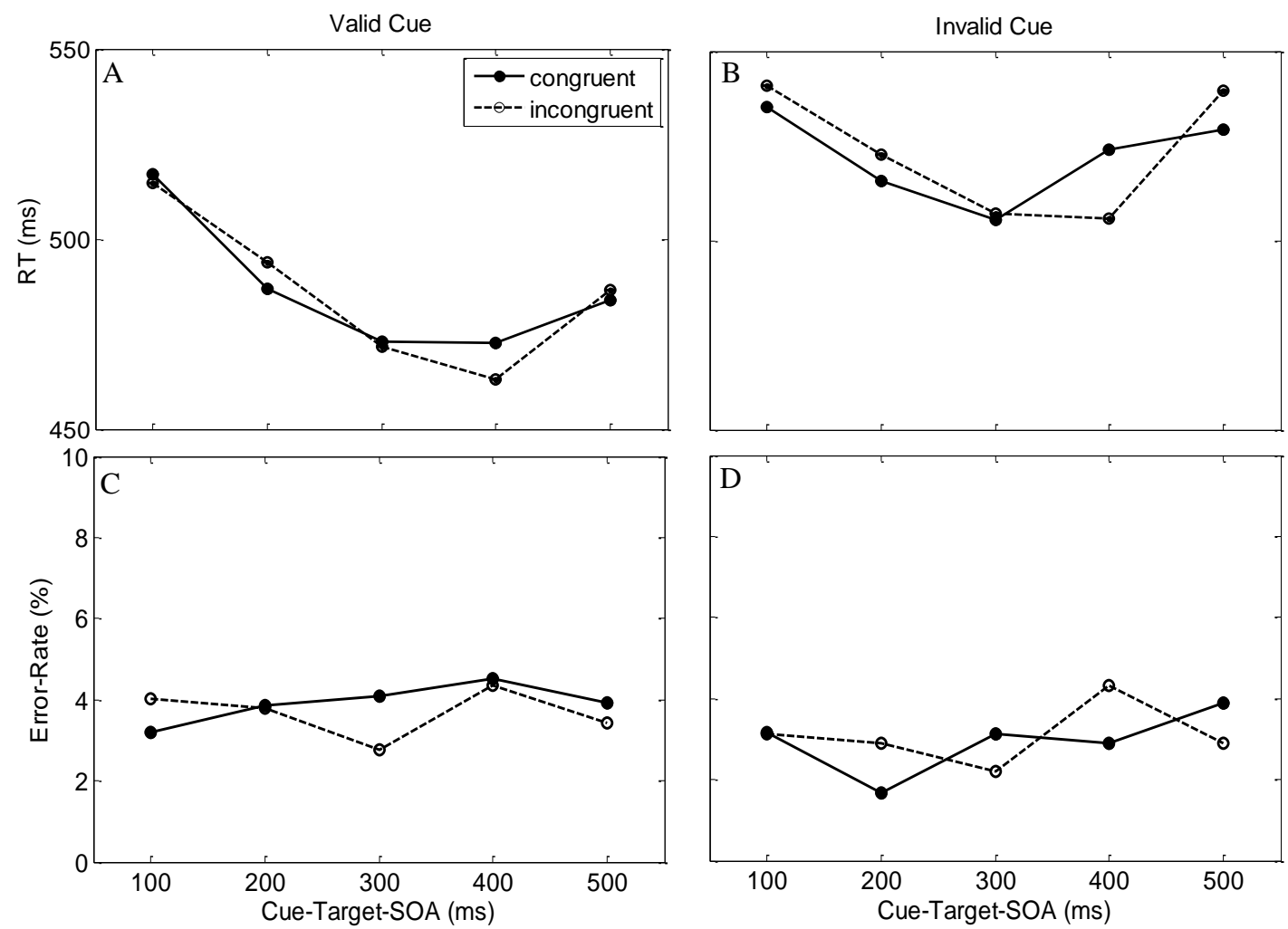

Figure 6.2 Results in Experiment 14. (A) RT with valid cues for congruent and incongruent trials as a function of cue-target SOA. (B) RT with invalid cues for congruent and incongruent trials as a function of cue-target SOA. (C) Error-rate with valid cues for congruent and incongruent trials as a function of cue-target SOA. (D) Errorrate with invalid cues for congruent and incongruent trials as a function of cue-target SOA. 
would expect differences in RT between different types of trials. Responses on trials with distractor should be faster and less dependent on cue-target SOA than responses on trials without distractor. If strategy effects transfer from trials with distractor to trials without distractor, responses on trials without distractor should be slower than responses on valid trials in Experiment 13.

\subsubsection{Method}

Participants. 11 students from the University of Goettingen (4 men, age 21-29, $\mathrm{M}=$ 24) completed 3 sessions of the experiment in exchange for $21 €$ or course credit. One additional participant was excluded because of very high error rates in all conditions.

Task. Figure 6.3 gives an example of the stimulation in one trial of Experiment 14. As in Experiment 13, participants were to discriminate between the letters A and B by pressing the "+" and "Enter" key on the number pad. They were informed that a target letter on the left side was predicted by a square cue and a target letter on the right side was predicted by a diamond cue and that cues were valid on each trial.

Apparatus and Stimuli. We used the same setup as in Experiment 13 with the exception that no eye movements were recorded in Experiment 14. Target letters were the same size and were presented at the same position as in Experiment 13, either to the left or to the right of fixation, along with either a relevant (A or B) or an irrelevant distractor letter (R) at the opposite side. Prime-cue SOA was fixed at $100 \mathrm{~ms}$ and cue-target SOA varied in 5 steps (100 ms, $150 \mathrm{~ms}, 200 \mathrm{~ms}, 300 \mathrm{~ms}, 400 \mathrm{~ms})$.

Design and Procedure. The first session was considered practice and not included in the analyses. The second and third session each comprised 5 blocks of 160 trials each. There was a break every 40 trials which participants could end at will. In each block, SOA was held 
constant and each combination of 2 primes, 2 cues, and 4 target arrays was presented 10 times in each session. Pooling across 2 sessions and 2 target positions there was a total of 80 congruent and 80 incongruent trials both with relevant distractor and irrelevant distractor with each SOA.

Analyses. No trials were excluded from analyses in Experiment 14. Untrimmed RT averages were computed for correct responses by each participant in each condition. Error rates were arc-sine transformed. Both dependent measures were analyzed using $2 \times 2 \times 5$ repeated-measures ANOVAs with factors Congruency, Distractor Relevance, and SOA.

\subsubsection{Results}

$R T$. Responses were faster on congruent trials $(593 \mathrm{~ms})$ than on incongruent trials

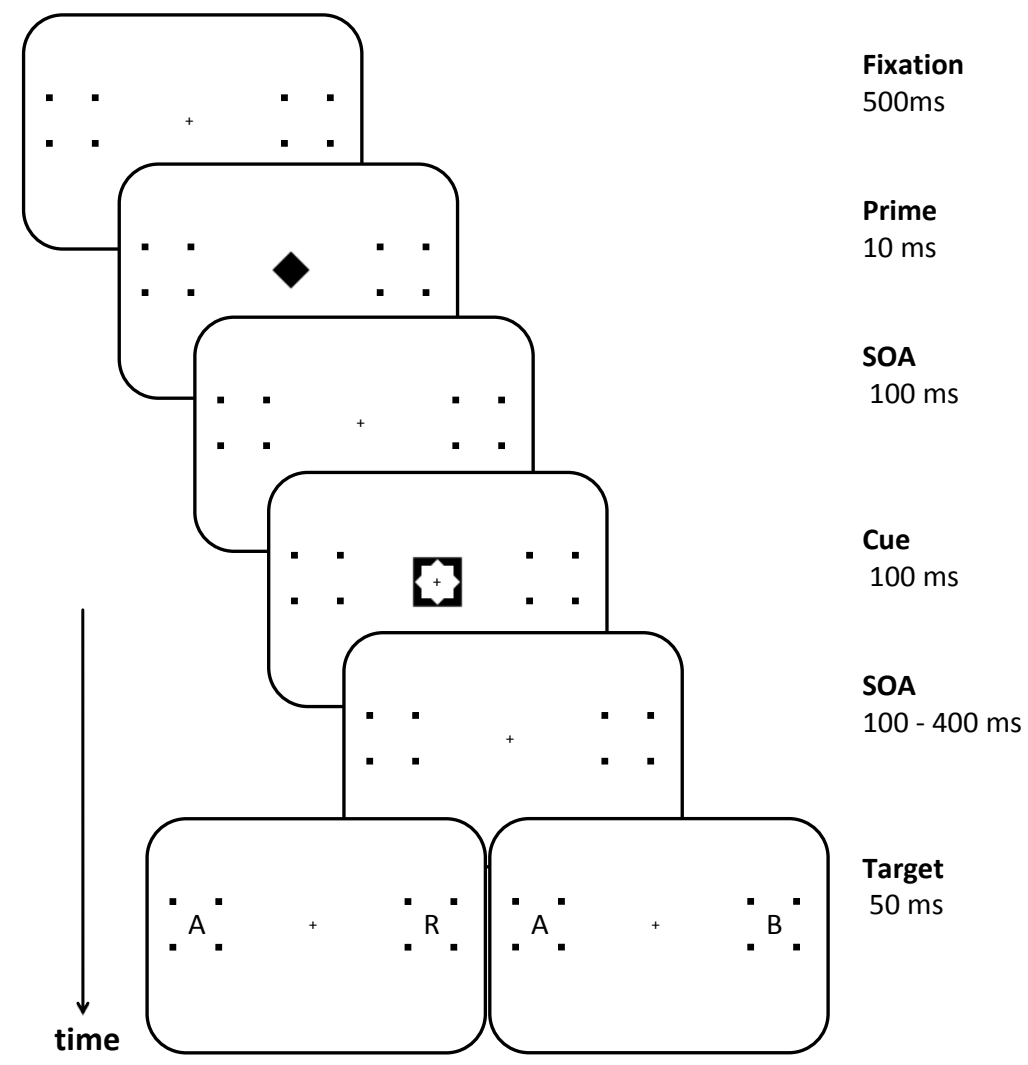

Figure 6.3 Sequence of events in a trial of Experiment 14. 
(607 ms), $F(1,10)=8.7, M S e=1036, p=.015$ and faster on trials with irrelevant distractor (573 ms) than on trials with relevant distractor $(625 \mathrm{~ms}), F(1,10)=6.9, M S e=22125, p=$ .025 (Figure 6.4A-B). Both of these factors interacted significantly with SOA, Congruency: $F(4,40)=4.7, M S e=449, p=.017$, Distractor Relevance: $F(4,40)=3.3, M S e=449, p=$ .035 , indicating that both effects were largest with short SOAs and smallest with $400 \mathrm{~ms}$ SOA: 66, 50, 60, 53, and $35 \mathrm{~ms}$, for the effect of Distractor Relevance (relevant - irrelevant), and 24, 26, 6, 15, -7 ms for the effect of Congruency (incongruent - congruent), with 100, $150,200,300$, and $400 \mathrm{~ms}$ SOA respectively. There was a main effect of SOA, $F(4,40)=$ 103.2, $M S e=1352, p<.001$, indicating that RT decreased with increasing cue-target SOA: $676,627,600,556$, and $535 \mathrm{~ms}$, with 100, 150, 200, 300, and $400 \mathrm{~ms}$ SOA respectively. There was neither a significant Congruency x Distractor Relevance interaction $F(1,10)=0.1$, $M S e=245, p=.728$ nor a significant Congruency x Distractror Relevance x SOA interaction $F(4,40)=0.6, M S e=340, p=.626$.

Response Errors. Participants commited more errors on trials with relevant distractors $(9.1 \%)$ than on trials with irrelevant distractors $(4.4 \%), F(1,10)=14.2, M S e=0.124, p=$ .004 (Figure 6.4C-D). No other main effect or interaction reached significance $(F<3, p>.05$, in all cases).

\subsubsection{Discussion}

With perfect cue validity, reliable priming effects were found as responses were faster on congruent than on incongruent trials. These priming effects were modulated by SOA as they were largest with short SOAs. This replicates results of Project 1. Responses were substantially slower on trials with relevant distractors than on trials with irrelevant distractors. This suggests that distractors were processed to a degree. However, priming effects were not affected by distractor relevance. The finding that prime-cue congruency and distractors 

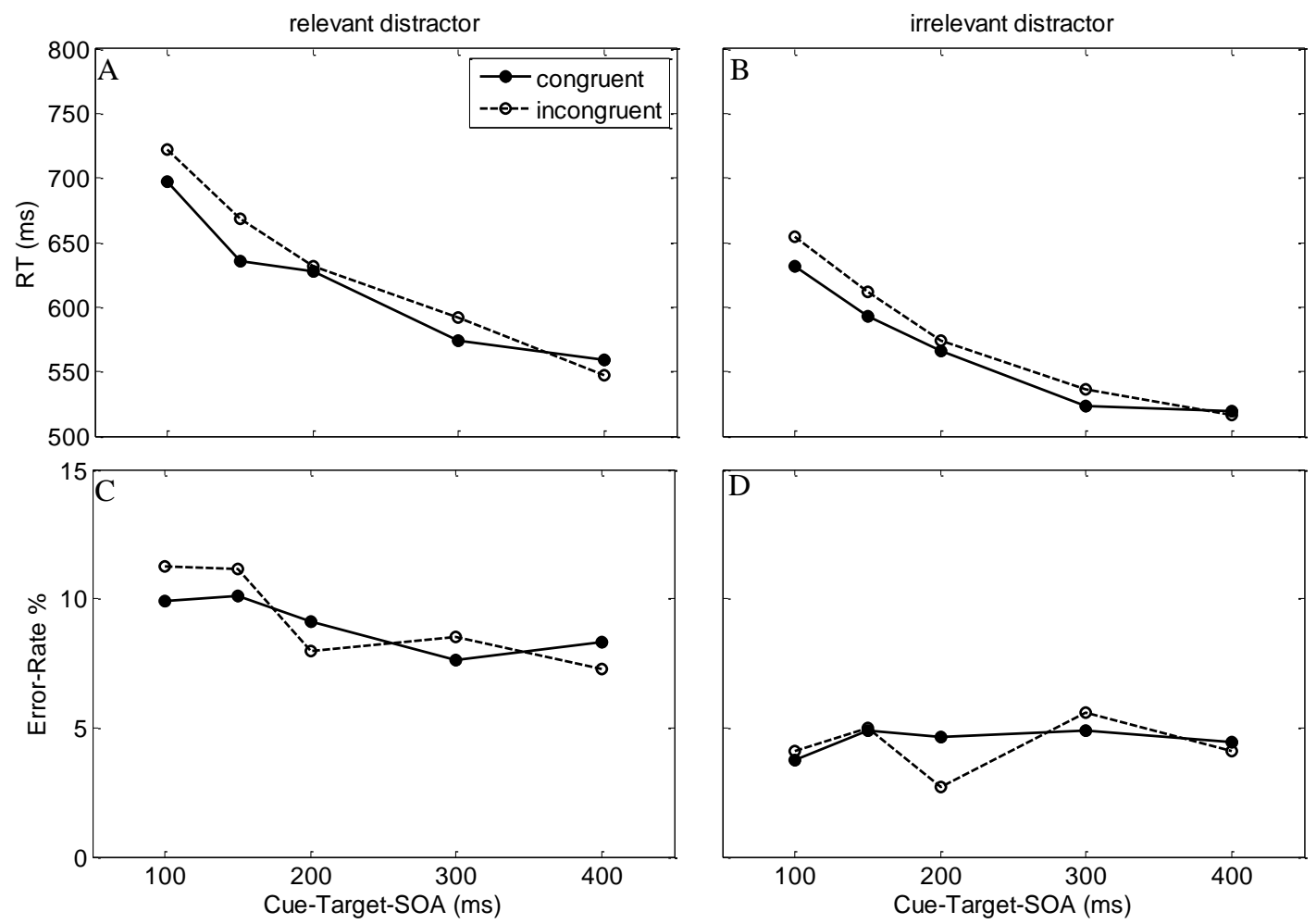

Figure 6.4 Results in Experiment 14. (A) RT with relevant distractors for congruent and incongruent trials as a function of cue-target SOA. (B) RT with irrelevant distractors for congruent and incongruent trials as a function of cue-target SOA. (C) Error-rate with relevant distractors for congruent and incongruent trials as a function of cue-target SOA. (D) Error-rate with irrelevant for congruent and incongruent trials as a function of cue-target SOA.

contribute additively to RT suggests that the two factors affect RT at different levels of processing. Mattler $(2003 ; 2005)$ found that distractor presence did not affect priming effects in a cue-priming task in which stimulus modality was cued. Thus, it seems unlikely that the absence of priming effects in Experiment 13 can be explained by the absence of a relevant distractor.

\subsection{General Discussion}

Overall, results suggest that cue validity plays an important role in spatial cue priming. The questions when and why this is the case should be addressed in future 
experiments. Responses in Experiment 14 were much slower than in Experiment 13 although trials with irrelevant distractors were comparable to trials in Experiment 13. It might be that the presence of trials with relevant distracters, which require the use of cues, led to differences between Experiments 13 and 14 in the way stimuli were processed on trials with irrelevant distractors. In Experiment 13, participants could in theory find the correct response without processing the cue whereas in Experiment 14 this was possible only on trials with irrelevant distractors. If this led participants to sequentially process first the cue and then the target on all trials this might lead to slower RT on trials with irrelevant distractors than would be possible without using the cue.

The irrelevant distractor stimulus was changed from $\mathrm{Z}$ to $\mathrm{R}$ between experiments. This was done to increase similarity between targets and distractors and thereby increase difficulty. We hoped that increasing difficulty would increase participants' motivation to use the cues even with irrelevant distracters, which should lead to larger priming effects. Unfortunately, however, this impedes comparison between Experiments 13 and 14 due to confoundation of irrelevant distactor identity and proportion of trials with valid cues. Considering the fact that we did find priming effects without any distractor stimulus in Experiment 8, it seems probable that the complete absence of priming effects in Experiment 13 is due to the presence of trials with invalid cues and not due to the task requiring little or no use of the cues because of easy discriminability of targets and distractor stimuli. Furthermore, RT with irrelevant distractors in Experiment 14 was significantly faster than with relevant distractors despite their being more similar to targets than the distractors in Experiment 13. Nevertheless, one might argue that the priming effects without distractor in Project 3 were found under conditions of increased perceptual load because it is a perceptually more demanding task to discriminate short and long bar targets than to 
discriminate $\mathrm{A}$ and $\mathrm{B}$. Therefore, further research is required to clarify how crucial cue validity really is for spatial cue-priming.

\subsection{Summary of Project 5}

In Project 5, we attempted to study cue-priming effects under conditions in which visible cues were sometimes invalid. Priming effects on trials with invalid cues might provide insight into the mechanisms of spatial cue-priming because on these trials, primes that are incongruent to the cue nevertheless correctly predict the target location. To our surprise, priming effects were absent in Experiment 13, whereas the expected effects of cue validity emerged. Experiment 14 tested whether the irrelevance of distractor letters in Experiment 13 can explain the absence of priming in Experiment 13. To this end, task relevance of the distractor letter was varied from trial to trial with cues that were always valid. Priming effects did not differ between the two types of distractors although a main effect of distractor relevance on $\mathrm{RT}$ and error rates suggests that trials with relevant distractors were more difficult than trials with irrelevant distractors. In sum, Project 5 shows that validity of visible cues plays an important role for cue-priming effects. At present, no definitive explanation can be given but it is speculated that priming effects depend crucially on cue validity because the presence of invalid cues leads to more controlled processing of cues which eliminates the influence of masked primes. 


\section{General Discussion}

In the reported experiments, a cue-priming procedure was successfully applied to spatial cueing tasks, showing that masked primes can affect response time and accuracy in a variety of tasks and on multiple levels of target processing. In sum, the present experiments provide evidence that masked symbolic primes can affect spatial attention. This is interesting because this type of stimuli requires a shift of attention away from the cue according to participants' expectations, which is a defining feature of endogenous or voluntary attention. This distinguishes the present findings from findings that masked stimuli can attract attention to their position (Mulckhuyse \& Theeuwes, 2010). In addition, the symmetrical symbolic cues used here do not have any attributes that have been found to induce unconscious or involuntary shifts of attention even with central cues, such as social relevance (Friesen \& Kingstone, 1998; Sato et al., 2007), spatial compatibility (Lambert et al., 2006; Ristic \& Kingstone, 2006), or overlearned associations (Guzzon, Brignani, Miniussi \& Marzi, 2010). In this sense, it can be concluded that masked primes can affect endogenous attention. This can be seen as an example for unconscious effects on executive control processes. However, simply presenting primes before visible cues in a spatial cueing task is not sufficient for these primes to have effects. Rather, it seems that certain conditions have to be met for spatial cuepriming effects to occur.

Effects of unconscious stimuli on executive control processes have been explained by assuming that stimulus processing takes form in a feed forward sweep which can reach prefrontal areas, but does not elicit consciousness because it lacks recurrent processing (Van Gaal \& Lamme, 2012). Beyond the basic finding of priming effects on endogenous spatial attention, the present experiments provide insight into the mechanisms at work in unconscious effects on spatial attention in particular and perhaps executive control processes in general. 


\subsection{Mechanisms of spatial cue-priming}

This section is intended to summarize the conclusions that can be drawn from the reported experiments about the mechanisms at work in spatial cue-priming. The summary has to account for the fact that there is no unambiguous picture emerging. Therefore, present evidence for and against candidate mechanisms is summarized and, where possible, suggestions for future experiments are made, which might help to clarify the picture.

As Mattler (2003) proposed, task demands in a cue-priming task can be separated into three separate stages: perceptual processing, central processing, and post-central processing. Theoretically, primes can have effects on each of these stages. These stages are organized hierarchically, which means that if primes affect processing at one stage this may propagate to all subsequent stages. Thus, if perceptual processing is faster on congruent than on incongruent trials, central and post-central processes may start earlier on congruent than on incongruent trials. This leads to attentional shifts occurring earlier, which may then affect target processing. This hierarchical organization makes it difficult to determine at which level processing is affected by primes. In the following, conclusions that can be drawn from the present experiments about priming effects on these three levels of processing are examined separately for each level.

Priming effects in a letter discrimination task with distractors are similar to cuepriming effects found with other tasks (Mattler, 2003; 2005) with regard to the effects of variation of prime-cue SOA and cue-target SOA. This resemblance suggests similar underlying processes. On the one hand, perceptual priming may play a role in all experiments as prime and cue stimuli were similar across all cue-priming studies. On the other hand, the letter discrimination task is similar to previous cue-priming studies because targets are 
presented along with relevant distractor stimuli and cues are needed to distinguish targets from distractors.

\subsubsection{The scope of spatial cue-priming}

In the present experiments spatial cue-priming effects were found in three different types of task that differ with regard to attentional demands. This suggests that attention operates differently in the different task and selects relevant information at different stages of processing (Luck \& Hillyard, 2000). Given that priming effects were found across all three tasks, it may seem reasonable to assume that attention affects the quality of perceptual target representations, which affects performance in all three tasks. However, there are differences in priming effects, which are probably due to cue-priming affecting different processes in different tasks.

The most reliable effects were found in a letter discrimination task, in which task relevant distractors were presented at the non-cued position. These effects were largest with short cue-target SOAs and did not depend entirely on perceptual similarity of primes and cues. Assuming that both target letters were relatively easy to perceive on a given trial, the main difficulty of the task most likely lies in deciding which letter should be reacted to. In contrast, in the signal detection experiments in Project 2, the task is perceptually difficult due to the weak target signal and masking. Attentional effects in this paradigm are hard to explain on the basis of a bias in selection of locations. In this task, priming effects were found with longer cue-target SOAs and only when prime and cue were perceptually similar. The bar length discrimination task used in Project 3 can be seen as a third type of task, as it requires stimulus discrimination instead of detection, and difficulty arises not because of a confusable distractor, but on perceptual level because the two possible targets (short and long) are perceptually similar. With this task, priming effects were relativlely small compared to the 
letter discrimination task and even reversed in one condition. However, they were found with perceptually dissimilar stimuli. Under the assumption that attention operates at early perceptual stages in the signal detection and bar discrimination tasks, but on later decisional stages in the letter discrimination task, results seem to be consistent with the assumption that spatial cue-priming is more effective at late levels of target processing than at early levels of processing. Thus, the presence and magnitude of spatial cue-priming depends not only on attributes of primes and cues, but also differs depending which level of attentional selection is employed in a specific task. This pattern may be due to varying degrees of priming of perceptual processing and priming of post-perceptual processing in the different tasks.

\subsubsection{Perceptual priming effects}

Part of cue-priming effects seems to depend on perceptual similarity between primes and cues. This could be due to perceptual repetition priming, which facilitates cue processing on congruent trials and impedes cue processing on incongruent trials. Similar effects of perceptual similarity have been reported in other priming experiments (Bodner \& Dypvik, 2005; Mattler, 2006). Perceptually, priming could be explained by repetition of simple visual features. If repetition priming accelerates cue processing on congruent trials and decelerates cue processing on incongruent trials, such early perceptual priming effects may propagate to target RT. Visual inspection of results from Project 3 (Experiments 9 and 10) suggests that early visual potentials (P1 and N1) induced by prime and cue, which were evident during the baseline time window in target-locked ERPs, were modulated by congruency. Such an effect of congruency was not apparent in cue locked ERPs in Experiment 8 where prime and cue were always presented at different locations and a star-shaped mask was used. This might hint at effects of similarity at early levels of perceptual cue processing on which exact spatial 
stimulus representations play a role. Results from Project 2 (Experiments 6 and 7) also suggest that perceptual priming is absent with the stimulus arrangement used in Experiment 8.

Further experiments are needed to clarify the mechanisms and limits of such perceptual priming. As it is reasonable to assume that perceptual priming is also prevalent in other paradigms, such studies need not involve spatial cue-priming. Ideally, a paradigm should be used that allows dissociation of perceptual priming and priming effects at other stages of processing. Then, perceptual similarity regarding critical attributes such as color, orientation, and size as well as distance between the stimuli and spatial compatibility should be gradually varied.

\subsubsection{Post-Perceptual Priming Effects}

Results of several experiments provide converging evidence that primes have access to post-perceptual levels of processing. First, in Project 1 priming effects were found when prime and cue were perceptually dissimilar. Second, priming effects in Experiment 8 occurred with cues and primes being presented at different locations. Although prime and cue symbols were similar in this experiment, it seems likely that the particular arrangement used in this experiment eliminates priming due to perceptual similarity because no priming effects were found with the same arrangement in Project 2 (Experiments 6 and 7). Third, free choice priming effects in Project 4 cannot be explained on basis of perceptual priming of cue processing, as processing speed of the free choice cue should not bias selection of sides. Fourth, there was considerable variation in priming effects across different tasks. Large and consistent priming effects emerged in experiments in which cues were needed to select between competing stimuli. In experiments where this was not the case, such as the signal detection experiments in Project 2, Experiment 8, and Experiment 13, priming effects were either small or absent. This dependence on specific task parameters would not be expected if 
priming effects are solely based on perceptual priming of cue processing, because perceptual cue processing is needed in all tasks.

At first glance, it may seem like these post-perceptual priming effects are restricted to letter discrimination tasks. However, assuming that perceptual priming effects are restricted to cases in which prime and cue are presented at the same location, results from Project 3 (Experiment 8), can be interpreted as evidence that primes can affect post-perceptual levels of processing in a task in which attention is probably allocated at early levels of target processing, as indicated by a modulation of target N1.

It remains to be determined on which mechanisms these post-perceptual priming effects are based. Earlier work distinguished between priming effects on central levels and those on post-central levels of processing (Mattler, 2006; Klapötke, 2011). On central levels, processing depends on current instructions and priming effects could arise from activation of task sets by primes. In the present experiments, this would mean that primes activate representations of attentional shifts to the associated position, but not yet initiate the shift itself. Thus, incongruent primes might induce conflict regarding the decision for one side and therefore delay that decision. The strongest assumptions about the effectiveness of spatial cue-priming would be that primes trigger the same processes as visible cues up to the initiation of shifts of attention. On congruent trials, primes might trigger the allocation of attention to the cued side which benefits performance, whereas performance on incongruent trials is impaired due to primes triggering shifts to the non-cued side. Assuming that cues in the present experiments trigger endogenous orienting of attention, which seems reasonable considering that they were centrally presented and symmetrical, this would suggest that primes can trigger endogenous orienting as well, meaning that they have access to postcentral levels of processing. 
By varying perceptual similarity in a choice RT task, we found that primes are likely processed beyond perceptual levels (see also Mattler, 2006), which means that they can at least affect decisions at a central level of processing. In a signal detection task, on the other hand, no such evidence for priming effects beyond perceptual levels was found. Perceptual processing is most likely similar in the two tasks since the same cues have to be identified. The finding that perceptually dissimilar primes had no effects in a signal detection task suggests that central processing differs between the two tasks, as the central processing is assumed to be independent of perceptual representations. However, if central processing is conceptualized as deciding for one side over the other, it seems that processing at this stage is similar in the two tasks as well. This makes localization of crucial differences between the two tasks at central stages seem questionable. One way to solve this problem might be to assume that processing differs at post-central levels of processing.

It has been proposed that primes can affect cue processing not only perceptually but also on basis of more complex conceptual associations (Reuss, Kiesel et al., 2011). Schneider and Logan (2006) employed a task switching paradigm in which multiple cues were used to indicate each task. They varied whether cues associated with the same task were semantically related words or unrelated words and found greater benefits of task repetition when the task was cued by two different related words on consequent trials than when it was cued by two unrelated words. From this, they suggested that there is associative or semantic priming between two task cues which are associated with the same task simply because being associated with the same task introduces associations between the cues. In the present spatial task, this argument may apply as well with respect to sides and not tasks.

This argument does not apply to free choice cue-priming of spatial attention where, on free choice trials, no cue is present to be primed (Reuss, Kiesel et al. 2011). However, free 
choice priming effects were only found with spatially compatible primes. This might suggest that priming with this type of stimuli extends beyond cue processing and affects spatial attention. It has been proposed that translation of cue stimuli to a spatial representation is a time consuming process that is facilitated by spatial correspondence (Lambert \& Duddy, 2002).

In order to conclude that primes can trigger orienting of attention, which would indicate priming on post-central levels of processing, priming effects would have to be shown to be independent of the effects of cues. This could be done by showing attentional benefits at non-cued but primed locations on incongruent trials. One way to do this could be using probe stimuli at non-cued positions which was attempted in Experiment 9 or by varying cue validity and showing that on trials on which cues are invalid incongruent primes lead to benefits in target processing. By extending the spatial cue-priming paradigm to more than two locations, one could assess whether benefits of primes are specific to the primed location. This might answer the question whether primes cause attentional orienting or a reduction of attentional cost of orienting to the cued side which may be caused by incongruent primes affecting cue processing.

\subsection{Alternative accounts for cueing effects in the present experiments}

\subsubsection{Primes affect attention by virtue of implicit learning}

Endogenous orienting has been proposed to be an executive control process and therefore linked to controlled and conscious processing. Thus, it seems appropriate to consider the possibility that priming effects on attention are not endogenous. Several findings suggest that implicit learning may play an important role in spatial cueing (Lambert, Naikar, McLachlan \& Aitken, 1999; Risko \& Stolz, 2010). Alternatively, it might be that, given 
enough learning experience, primes can affect attention by virtue of the same mechanisms as arrows and eye-gaze. It has been proposed that the distinctiveness of these cues is based on extensive learning experience from everyday situations. With enough learning similar automatic orienting might be found with arbitrary cues. Results from Guzzon, Brignani, Miniussi \& Marzi (2010) support this notion.

It is important to note that in most experiments there was no relation between prime symbols and target location (this is not true for experiments in which perceptual similarity was varied in which primes predicted target location with $66 \%$ accuracy). Therefore, participants would not benefit from associating primes with either side. It follows that prime symbols should only be associated to a side and affect attention accordingly by virtue of the predictive cues with which they share perceptual attributes. This seems to argue against an implicit learning account. Beyond this, priming effects in general did not increase over the course of the experiments, which is what one would expect if learning is a critical factor. Zhou \& Davis (2012) found priming effects on task selection based on implicit learning of associations between primes and task. However, they proposed that priming effects with explicit association of stimulus and task, like in the present experiments, result from different processes. In sum, it seems unlikely that the present priming effects are based on implicit learning of associations of primes and sides.

\subsubsection{Task switching and compound cue retrieval}

Priming effects in Project 1 as well as the free choice priming effects in Project 4 exhibit remarkable similarity to effects found with direct priming of motor responses. In these experiments the combination of cue and target stimuli specifies the correct response. The letter discrimination task used mainly in Project 1 was designed to study spatial attention while using a paradigm similar to other cue-priming studies. As other cue-priming paradigms 
used tasks similar to those employed in task switching studies, it is possible that similar underlying mechanisms play a role in cue-priming. This could mean that in this task, participants do not use the cues to enhance processing of stimuli presented at the cued side, but instead to update the current task set which consists of the necessary stimulus-response mappings. Thus, in Project 1 participants might not have focused on the letter at the cued position but instead processed both letters as one of two possible targets and then mapped these to the responses according to the cue. This updating of task sets is different from the preparatory processes usually studied in spatial cueing although the two possible targets differ only regarding a spatial dimension. In a different approach to task switching effects, it has been proposed that participants in task switching experiments combine cue and target to socalled compound cues, which are then used to retrieve the correct response from memory (Logan \& Bundesen, 2003). In this conception, the letter discrimination task is only slightly more complex than a simple target discrimination task typically used in response priming, as instead of only one parameter two parameters have to be set and combined to retrieve a response. Applying the idea of direct parameter specification, primes could be used to set the same parameter as cues. However, task switching accounts are only applicable in experiments in which cues are needed for response selection. It cannot explain priming effects on signal detection and with single targets. Thus, it cannot account for all priming effects reported here. However, it might be that priming effects are based on different underlying processes in different experiments and the large and consistent priming effects in letter discrimination experiments could be in part based on updating of task sets or direct parameter specification of a part of compound cues. 


\subsection{Open questions}

\subsubsection{Limits of spatial cue-priming}

Can masked primes directly trigger shifts of spatial attention? Does cue-priming affect early levels of target processing. Ideally, this question could be answered by disentangling the contributions of primes to shifts of attention from the contribution of visible cue stimuli. This can be done either by showing effects of primes on stimuli that are presented at non-cued positions or by omitting the visible cue altogether (see Reuss, Kiesel et al., 2011, for such an approach). The former approach was employed in two experiments of the present study. On the one hand, by varying cue validity, which proved difficult as it led to absence of priming effects and, on the other hand, by recording ERPs to irrelevant probe stimuli at non-cued positions, which were found not to be enhanced by primes with short cue-probe SOAs. However, both of these approaches might potentially prove fruitful in answering the question provided methodical refinement. If a method was devised to study cue-priming under conditions of less than $100 \%$ cue validity - perhaps by increasing participants' motivation to use the cues by increasing task difficulty or by minimizing the relative amount of invalid trials - it would provide the interesting opportunity to study cue-priming effects at the non-cued position. Priming effects in a probe experiment might be made more likely to occur by optimizing cue-target and cue-probe SOAs. Across experiments, priming effects on early levels of target processing were found with cue-target SOAs longer than the one used in the Experiment 9. In addition to the use of a longer cue-probe SOA, the task should be modified to increase perceptual processing demands, for instance by employing a bar discrimination task or a luminance detection task. This 
might lead to attention affecting earlier processing stages than in the letter discrimination task.

The spatial cue-priming paradigm as employed in the present experiments is restricted by the use of a separate visible cue with $100 \%$ validity and a limited range of both prime-cue and cue-target SOAs. Thus, it is unclear to which degree the present results allow generalizations about the role of conscious processing in other spatial cueing paradigms regarding time course and effects of validity in unconscious cueing.

\subsubsection{The role of cue-target SOA}

The pattern of results does not seem clear with regard to the role of cue-target SOA in spatial cue-priming. On the one hand, we found that priming effects decrease with increasing cue-target SOA in a letter discrimination task. On the other hand, priming effects in tasks that are more typical for spatial attention, like the signal detection task in Project 2 and the bar discrimination task without distractor in Project 3 were mainly found with longer cue-target SOAs from 300 to $400 \mathrm{~ms}$. This pattern of results can be explained by assuming different attentional mechanisms in two types of task. Luck and colleagues (1996) proposed that the minimal cue-target SOA needed for spatial cueing effects is dependent on the task. In tasks that require allocation of attentional resources prior to target presentation longer cue-target SOAs are needed because resource allocation is a time consuming process. Cueing effects due to a reduction of spatial uncertainty, on the other hand, are possible with shorter cue-target SOAs. In other cue-priming experiments (Mattler, 2005), effects have been found to decay with increasing cue-target SOA. However, it seems that these previously studied effects were more similar to the present letter discrimination effects. Therefore, it may be that priming effects in other tasks exhibit different time courses. 
At first glance, results of Experiment 10 could be taken as a replication of the priming effects in Experiment 8 of the same project with a shorter cue-target SOA (158 ms). However, in this experiment trials with only one bar target were mixed with trials with two dissimilar bar targets, one on each side of the screen. From participants' reports, it seems that on these two target trials the length of each bar can be inferred easily from comparison with the other bar. This might place the main difficulty of the task at finding the correct response rather than finding the length of a single target bar. This makes the task more similar to the letter discrimination task. This is supported by the result that reactions were slower with two targets than with one target, despite participants' reports about the former being easier. This emphasis on response selection might even affect responses on one target trials if trials are mixed.

In addition, different priming effects may result if trials with relatively long cue-target SOA are mixed with trials with short SOA. In Experiment 10 reversed priming effects were found in a condition that was otherwise similar to Experiment 8, in which positive priming effects were found. Replication of Experiment 10 with blocks of only one target trials and blocks of only two target trials might clarify whether it is the variation of cue-target SOA between trials that led to this reversal or other factors such as the presence of distractors on some trials.

\subsubsection{The role of cue validity}

Results from Project 5 suggest that cue validity is a critical factor in spatial cuepriming. Reducing prime validity to $80 \%$ of trials led to complete absence of priming effects although considerable cueing effects were found. Thus, it seems that it is not sufficient for cue-priming effects to present primes that share critical attributes with effective spatial cues. Rather, specific features of the cues seem to determine the effects of primes. This suggests 
that priming effects depend on the present of a certain task context provided by the cues, which may be seen as an instance of context effects on unconscious cognition. This idea relates to the direct parameter specification (Neumann \& Klotz, 1994) and action trigger accounts (Kunde, Kiesel \& Hoffmann, 2003) devised to explain response priming. The reasons for the crucial role of validity remain to be determined. The fact that there were effects of cue validity in Experiment 13 makes it seem that the absence of priming is due to a lack of motivation or attention to the cues. Results of Experiment 14 also seem to rule out distractor relevance as the deciding factor, although a replication with blocked presentation of trials with relevant and irrelevant distractors may be warranted to exclude the possibility that effects carry over from one type of trials to the other. Instead, it might be that primes can only affect the same processes as cues if participants rely strongly on the cues and believe there are no costs involved in following the cues. When there is a possibility that cues are invalid additional control processes might come into play that reduce or eliminate the impact of masked stimuli.

\subsubsection{The role of spatial compatibility}

In addition, central processing seems to be affected by spatial compatibility between prime and cue stimuli on the one hand, and target positions on the other hand. This finding is in line with the fact that previous effects of masked stimuli on spatial attention have only been reported with asymmetrical stimuli. An explanation for this is offered by the spatial correspondence learning hypothesis by Lambert and colleagues, who suggest that spatial correspondence between perceptual features of cue stimuli and target locations is a critical factor in spatial cueing because "it appears that translating the cue into a spatial representation is the component that involves a relatively slow effortful process." (Lambert \& Duddy, 2002, 
p. 316). This translating is a time consuming process with symmetrical cues that is facilitated with spatially compatible cues.

Spatial compatibility is not only important in cueing of attention but also seems to facilitate priming of motor responses (Mattler \& Palmer, 2012). Further attempts to study the contribution of spatial compatibility in free choice priming as well as spatial cueing might help to distinguish priming of cue processing from priming of attention as well as clarify whether the advantage of spatial compatibility is related to unconscious processing. To this end, spatially compatible and symmetrical cues and primes could be employed in the same experiment with combination of those two categories on some trials. If effects of spatially compatible primes extends beyond priming of cue processing they should be effective regardless of whether the cue is spatially compatible as well, whereas effects of symmetrical primes might depend on similarity and are thus more effective when the cue is also symmetrical.

\subsubsection{The role of prime visibility}

With the metacontrast masking procedure used in the present experiments, it proved difficult to achieve complete masking of primes. In forced choice prime identification tasks, participants were able to discriminate primes with higher than chance accuracy. However, priming effects did not correlate with prime visibility in most cases. It seems important to note that we adopted a very thorough assessment of prime recognition performance in our experiments. We made sure that prime recognition sessions were comparable to priming session in length and procedure. This is a more conservative test than what is done in many other experiments on masked priming which only administer a few blocks of prime recognition trials at the end of a priming session (e.g., Reuss, Kiesel, et al. 2011). It is important to measure prime visibility with sufficient test power, considering that proving that 
primes were not visible essentially requires assumption of the null hypothesis that recognition performance does not differ from chance level. Other cue-priming studies (e.g., Lau \& Passingham, 2007; Mattler, 2003) have used dissociation of priming effects and prime visibility with varying prime-cue SOAs to provide evidence for the independence of priming and prime visibility (Schmidt \& Vorberg, 2006). In the present experiments, decreasing prime recognition performance with increasing prime-cue SOA did occur in some participants, but was too rare to statistically support similar dissociation.

Is spatial cue-priming only possible with visible primes? Evidence from the reported experiments suggests that cue-priming effects do not correlate with prime visibility. However, prime recognition performance was better than chance in all experiments which leaves the question formulated above open. It might therefore be warranted to replicate some of the reported experiments with improved masking. At present, however, it is unclear which procedures should be used to increase masking without diminishing priming effects. One possible method would be to use conditions that lead to decreasing prime visibility with increasing SOA, a result which has been obtained with similar stimuli as those used here but was not stably found in the present experiments. Unfortunately, it is unclear which conditions favor such a time course and there seem to be considerable inter-individual differences (Albrecht, Klapötke \& Mattler, 2010). Another approach to improving masking would be to use different stimuli. This might be done by reducing stimulus contrast, inserting a separate mask between prime and cue, using a different means of masking than metacontrast masking, e.g., pattern masking, using more complex symbols, or varying the position at which primes are presented. The latter two methods were combined and used in three experiments here and successfully reduced prime visibility. However, priming effects were mostly absent and in the one condition in which they were not, recognition performance was again above chance. It 
seems unlikely that the other mentioned methods to reduce prime visibility would leave priming effects unaffected.

\subsection{Integration}

Integrating preconditions and characteristics across different tasks might lead to a comprehensive model of spatial cue-priming. However, at present integration seems difficult due to a complex pattern of results from several experiments and tasks. Nevertheless, the present experiments allow conclusions about some mechanisms by which masked primes can affect performance in the present spatial cueing tasks. It seems warranted to dissect cognitive processes needed in the present tasks into several stages. Potentially, primes might have effects on each of the following stages: perceptual cue processing, selection of one side, allocation of attention, perceptual target processing, and response selection. Earliest effects of primes seem to be based on perceptual similarity between primes and cues. These effects were most likely present in all of the present experiments in which simple square and diamond stimuli were used, except when the predictive validity of the cues was reduced. After perceptual processing, primes and cues need to be translated to a spatial representation to select one side. This translation is needed in each of the employed tasks and might be facilitated by the use of spatially compatible stimuli. Subsequently, primes can seemingly affect the allocation of attention. However, these post-perceptual priming effects seem to depend on the task that has to be done. Evidence for post-perceptual priming effects were found only in discrimination tasks and were especially large when there were trials with relevant distractor stimuli. Speculatively, primes have access to post-perceptual processes in discrimination tasks but not in detection tasks. In addition, it might play a role whether the effect of cues lies in an early modulation of (perceptual) target processing or the cue affects decisions on later stages, e.g., the decision which of two potential targets is used to specify the 
response. In the former case, target processing is possible without cue processing as the target provides all relevant information. Under such conditions, priming effects seem to be either small or absent. In the latter case, priming effects are larger. This might be because primes and cues in this type of task modulate response selection more than target processing because cues specify which of two stimuli has to be responded to, and measures of RT may thus be affected by cue processing time especially with short cue-target SOAs. This may even extend to trials with unambiguous targets in Experiment 10 and Experiment 14. If the presence or relevance of distractors varies from trial to trial participants always have to expect to need the cue before seeing the target. This might lead to a transfer of priming effects on cue processing duration even to trials on which cues are not needed, which may explain why neither factor affects the magnitude of priming effects in these experiments. Unconscious processing has been associated with effects of fast feed forward processing (van Gaal \& Lamme, 2012). Findings that masked stimuli can affect cognitive control processes suggest that feed forward processing reaches higher brain areas and even the prefrontal cortex. Applying this idea to the present experiments, the present results might allow conclusions about the extent of such fast feed forward processing. Especially the finding that post-perceptual priming effects were absent in Project 2 suggests limitations. Perceptually demanding tasks like this might require allocation of resources at an earlier level than in discrimination tasks. Thus, feed forward processing of primes has to reach decision levels in both tasks and then extend to early perceptual levels only in the case of perceptually demanding tasks where effects on decision are minimal. This might not be the case in the letter discrimination task where effects on the decision for a side are all that is needed to explain priming effects. 


\subsection{Summary of conclusions}

In summary, the present work shows that spatial attention can be affected by centrally presented masked primes. We assume that this is an instance of unconscious effects on executive control processes, because the allocation of attention according to central symbolic cues is an executive control process and the priming effects seem to be independent of prime visibility, although we did not succeed in realizing conditions of complete masking. However, these cue-priming effects on spatial attention seem to be dependent on several factors which impede conclusions about the underlying mechanisms. Overall, it seems that much although not all of the spatial cue-priming effect results from priming of cue processing rather than priming of attention shifts. When task performance (especially RT) depends on fast cue processing, which is the case in the letter discrimination task where the cue is needed to select the correct response, large priming effects emerge, especially with short cue-target SOAs. This is at odds with findings from spatial cueing studies in which effects of cue validity have been found to increase with cue-target SOA. In other tasks, in which performance probably depends more on allocation of attentional resources prior to target onset, priming effects were found mostly with longer cue-target SOAs.

The finding that response selection processes seem to be more readily affected by masked primes than perceptual representation of targets seems to make sense considering evolutionary advantages of fast reactions to external stimuli. It seems more important to respond fast by acting than to direct attention in order to detect what exactly is going on.

In conclusion, masked primes can modulate spatial attention in a similar way as other mental operations. However, spatial cue-priming effects are short lived and restricted by several task parameters such as perceptual similarity, spatial compatibility and the task 
context set by cue validity. Thus, fears of subliminal manipulation of attention in an everyday setting are most likely unwarranted. 


\section{References}

Albrecht, T., Klapötke, S., \& Mattler, U. (2010). Individual differences in metacontrast masking are enhanced by perceptual learning. Consciousness and Cognition, 19(2), 656-666. doi:10.1016/j.concog.2009.12.002

Albrecht, T., \& Mattler, U. (2010). Individual differences in metacontrast masking: A call for caution when interpreting group data. Consciousness and Cognition, 19(2), 672-673. doi:10.1016/j.concog.2010.03.010

Anllo-Vento, L. (1995). Shifting attention in visual space: the effects of peripheral cueing on brain cortical potentials. The International journal of neuroscience, 80(1-4), 353-370. Retrieved from http://informahealthcare.com/loi/nes

Baars, B. J. (1993). A Cognitive Theory of Consciousness. Cambridge University Press.

Bargh, J. A., Chen, M., \& Burrows, L. (1996). Automaticity of social behavior: Direct effects of trait construct and stereotype activation on action. Journal of Personality and Social Psychology, 71(2), 230-244. doi:10.1037//0022-3514.71.2.230

Bermeitinger, C., Goelz, R., Johr, N., Neumann, M., Ecker, U. K. H., \& Doerr, R. (2009). The hidden persuaders break into the tired brain. Journal of Experimental Social Psychology, 45(2), 320-326. doi:10.1016/j.jesp.2008.10.001

Bodner, G., \& Dypvik, A. (2005). Masked priming of number judgments depends on prime validity and task. Memory \& Cognition, 33(1), 29-47. doi:10.3758/BF03195295

Botvinick, M. M., Cohen, J. D., \& Carter, C. S. (2004). Conflict monitoring and anterior cingulate cortex: an update. Trends in Cognitive Sciences, 8(12), 539-546. doi:10.1016/j.tics.2004.10.003 
Brignani, D., Guzzon, D., Marzi, D. A., \& Miniussi, C. (2009). Attentional orienting induced by arrows and eye-gaze compared with an endogenous cue. Neuropsychologia, 27, $370-381$.

Broadbent, Donald E. 1958. Perception and Communication. New York: Pergamon Press.

Carrasco, M., Ling, S. \& Read, S. (2004). Attention alters appearance. Nature Neuroscience, 7, 308-313.

Cheesman, J., \& Merikle, P. M. (1984). Priming with and without awareness. Perception \& Psychophysics, 36(4), 387-395.

Chiu, Y.-C., \& Yantis, S. (2009). A domain-independent source of cognitive control for task sets: shifting spatial attention and switching categorization rules. The Journal of Neuroscience, 29, 3930-3938.

Cohen, M. A., Cavanagh, P., Chun, M. M., \& Nakayama, K. (2012). The attentional requirements of consciousness. Trends in Cognitive Sciences, 16(8), 411-417. doi:10.1016/j.tics.2012.06.013

Cole, G. G., \& Kuhn, G. (2010). What the experimenter's prime tells the observer's brain. Attention, Perception, \& Psychophysics, 72, 1367-1376.

Corbetta, M., \& Shulman, G. L. (2002). Control of goal-directed and stimulus-driven attention in the brain. Nature Reviews Neuroscience, 3(3), 201-215. doi:10.1038/nrn755

Custers, R., \& Aarts, H. (2010). The Unconscious Will: How the Pursuit of Goals Operates Outside of Conscious Awareness. Science, 329(5987), 47-50. doi:10.1126/science.1188595 
De Pisapia, N., Turatto, M., Lin, P., Jovicich, J., \& Caramazza, A. (2011). Unconscious Priming Instructions Modulate Activity in Default and Executive Networks of the Human Brain. Cerebral Cortex, 22, 639-649.

Dehaene, S. (2008). Conscious and nonconscious processes. Distinct forms of evidence accumulation? In C. Engel \& W. Singer (Eds.), Better Than Conscious? Decision Making, the Human Mind, and Implications For Institutions (pp. 22-49). Cambridge, MA: MIT Press.

Dehaene, S., \& Changeux, J.P. (2011) Experimental and theoretical approaches to conscious processing. Neuron, 70, 200-227.

Dehaene, S., \& Naccache, L. (2001). Towards a cognitive neuroscience of consciousness: basic evidence and a workspace framework. Cognition, 79(1-2), 1-37. doi:10.1016/S0010-0277(00)00123-2

Dehaene, S., Naccache, L., Clec'H, G. L., Koechlin, E., Mueller, M., Dehaene-Lambertz, G., ... Bihan, D. L. (1998). Imaging unconscious semantic priming. Nature, 395(6702), 597-600. doi:10.1038/26967

Dennett, D.C. (2009) Multiple drafts model. In The Oxford Companion to Consciousness. (Bayne, T. et al., eds), pp. 452-454, Oxford University Press.

Desimone, R., \& Duncan, J. (1995). Neural mechanisms of selective visual attention. Annual Review of Neuroscience, 18, 193-222.

Deutsch, A, J., \& Deutsch, D. (1963). Attention: Some Theoretical Considerations. Psychological Review, 70(1), 80-90. doi:10.1037/h0039515

Duncan, J. (1980). The demonstration of capacity limitation. Cognitive Psychology, 12, 7596. 
Duncan, J. (2006) EPS Mid-Career Award 2004 - Brain mechanisms of attention. Quarterly Journal of Experimental Psychology, 59(1), 2-27.

Eimer, M. (1993). Spatial cueing, sensory gating and selective response preparation: an ERP study on visuo-spatial orienting. Electroencephalography and Clinical Neurophysiology/Evoked Potentials Section, 88(5), 408-420. doi:10.1016/01685597(93)90017-J

Eimer, M. (1994). "Sensory gating” as a mechanism for visuospatial orienting: Electrophysiological evidence from trial-by-trial cuing experiments. Perception \& Psychophysics, 55(6), 667-675. doi:10.3758/BF03211681

Eimer, M. (1999). Facilitatory and inhibitory effects of masked prime stimuli on motor activation and behavioural performance. Acta Psychologica, 101(2-3), 293-313. doi:10.1016/S0001-6918(99)00009-8

Eimer, M. \& Schlaghecken, F. (1998). Effects of masked stimuli on motor activation: Behavioural and electrophysiological evidence. Journal of Experimental Psychology: Human Perception and Performance, 24, 1737-1747.

Esterman M, Chiu YC, Tamber-Rosenau BJ, \& Yantis, S. (2009). Decoding cognitive control in human parietal cortex. Proceedings of the National Academy of Sciences, 106, 17974-17979.

Friesen, C. K., \& Kingstone, A. (1998). The eyes have it! Reflexive orienting is triggered by nonpredictive gaze. Psychonomic Bulletin \& Review, 5, 490-495.

Frischen, A., Smilek, D., Eastwood, J. D., \& Tipper, S. P. (2007). Inhibition of return in response to gaze cues: The roles of time course and fixation cue. Visual Cognition, 15(8), 881-895. doi:10.1080/13506280601112493 
Guzman-Martinez, E., Leung, P., Franconeri, S., Grabowecky, M., \& Suzuki, S. (2009). Rapid eye-fixation training without eyetracking. Psychonomic Bulletin \& Review, $16(3), 491-496$.

Guzzon, D., Brignani, D., Miniussi, C., \& Marzi, C. A. (2010). Orienting of attention with eye and arrow cues and the effect of overtraining. Acta Psychologica, 134(3), 353-362. doi:10.1016/j.actpsy.2010.03.008

Handy, T. C., \& Mangun, G. R. (2000). Attention and spatial selection: Electrophysiological evidence for modulation by perceptual load. Perception \& Psychophysics, 62(1), 175186. doi:10.3758/BF03212070

Harter, M. R., Miller, S. L., Price, N. J., LaLonde, M. E., \& Keyes, A. L. (1989). Neural Processes Involved in Directing Attention. Journal of Cognitive Neuroscience, 1(3), 223-237. doi:10.1162/jocn.1989.1.3.223

Hawkins, H., Hillyard, S., Luck, S., Mouloua, M., Downing, C., \& Woodward, D. (1990). Visual attention modulates signal detectability. Journal of Experimental Psychology: Human Perception and Performance, 16, 802-811.

Hawkins, H., Shafto, M., \& Richardson, K. (1988). Effects of target luminance and cue validity on the latency of visual detection. Attention, Perception, \& Psychophysics, 44, 484-492.

Heinze, H. J., Luck, S. J., Mangun, G. R., \& Hillyard, S. A. (1990). Visual event-related potentials index focused attention within bilateral stimulus arrays. i. evidence for early selection. Electroencephalography and clinical Neurophysiology, 75, 511-527.

Heinze, H. J., Mangun, G. R., Burchert, W., Hinrichs, H., Scholz, M., Münte, T. F., ... Hillyard, S.A. (1994). Combined spatial and temporal imaging of brain activity during 
visual selective attention in humans. Nature, 372(6506), 543-546.

doi:10.1038/372543a0

Hillyard, S. A., \& Anllo-Vento, L. (1998). Event-related brain potentials in the study of visual selective attention. Proceedings of the National Academy of Sciences, 95, 781 -787. Retrieved from http://www.pnas.org

Hillyard, S. A., Vogel, E. K., \& Luck, S. J. (1998). Sensory gain control (amplification) as a mechanism of selective attention: electrophysiological and neuroimaging evidence. Philosophical Transactions of the Royal Society of London. Series B: Biological Sciences, 353(1373), $1257-1270$.

Hommel, B. (2007). Consciousness and control: Not identical twins. Journal of Consciousness Studies, 14, 155-176.

Hopf, J.-M., \& Mangun, G. R. (2000). Shifting visual attention in space: an electrophysiological analysis using high spatial resolution mapping. Clinical Neurophysiology, 111(7), 1241-1257. Retrieved from http://www.clinph-journal.com

Hummel, J.E. (2001). Complementary solutions to the binding problem in vision: Implications for shape perception and object recognition. Visual Cognition, 8, 489517.

Jack, A. I., \& Shallice, T. (2001). Introspective physicalism as an approach to the science of consciousness. Cognition, 79(1-2), 161-196. doi:10.1016/S0010-0277(00)00128-1

James, W. (1950).The principles of psychology (Vol. 1). New York, NY: Dover. (Original work published 1890). 
Jonides, J. (1981). Voluntary vs. automatic control over the mind's eye's movement. In J. B. Long \& A. D. Baddeley (Eds.), Attention and performance, IX (pp. 187-203). Hillsdale, NJ: Erlbaum.

Kaernbach, C. (1991) Simple adaptive testing with the weighted up-down method, Perception \& Psychophysics, 45, 227-229.

Kahneman, D., \& Treisman, A. (1984). Changing views of attention and automaticity. In R. Parasuraman, D.R. Davies \& J. Beatty (Eds.), Variants of attention (pp. 29-61). New York : Academic Press.

Karremans, J. C., Stroebe, W., \& Claus, J. (2006). Beyond Vicary's fantasies: The impact of subliminal priming and brand choice. Journal of Experimental Social Psychology, 42(6), 792-798. doi:10.1016/j.jesp.2005.12.002

Kentridge, R. W., Heywood, C. A., \& Weiskrantz, L. (1999). Attention without awareness in blindsight. Proceedings of the Royal Society of London. Series B: Biological Sciences, 266, 1805-1811.

Kiesel, A., Wagener, A., Kunde, W., Hoffmann, J., Fallgatter, A. J., \& Stöcker, C. (2006). Unconscious manipulation of free choice in humans. Consciousness and Cognition, 15(2), 397-408. doi:10.1016/j.concog.2005.10.002

Kingstone, A., Smilek, D., Ristic, J., Friesen, C. K., \& Eastwood, J. D. (2003). Attention, researchers! It is time to take a look at the real world. Current Directions in Psychological Science, 12, 176-180.

Klapötke, S. (2011). Effekte maskierter visueller Stimuli auf die Ausführung von konkurrierenden motorischen Reaktionen und kognitiven Aufgaben. (Doctoral dissertation). Georg-August-Universität Göttingen, Göttingen. Available from: http://webdoc.sub.gwdg.de/diss/2011/klapoetke/klapoetke.pdf 
Klapötke, S., Krüger, D., \& Mattler, U. (2011). A PRP-study to determine the locus of target priming effects. Consciousness and Cognition, 20(3), 882-900. doi:10.1016/j.concog.2011.04.008

Klapp, S. T., \& Haas, B. W. (2005). Nonconscious influence of masked stimuli on response selection is limited to concrete stimulus-response associations. Journal of Experimental Psychology: Human Perception and Performance, 31, 193-209.

Klotz, W., \& Neumann, O. (1999). Motor activation without conscious discrimination in metacontrast masking. Journal of Experimental Psychology: Human Perception and Performance, 25(4), 976-992. doi:10.1037/0096-1523.25.4.976

Koch, C., \& Tsuchiya, N. (2007). Attention and consciousness: Two distinct brain processes. Trends in Cognitive Sciences, 11, 16-22

Krüger, D., Klapötke, S., Bode, S., \& Mattler, U. (2012). Neural correlates of control operations in inverse priming with relevant and irrelevant masks. NeuroImage, 64, 197-208. DOI:10.1016/j.neuroimage.2012.09.018

Kunde, W., Kiesel, A., \& Hoffmann, J. (2003). Conscious control over the content of unconscious cognition. Cognition, 88, 223-242.

LaBerge, D., \& Brown, V. (1989). Theory of attentional operations in shape identification. Psychological Review, 96(1), 101-124. doi:10.1037/0033-295X.96.1.101

Lambert, A. J., \& Duddy, M. (2002). Visual orienting with central and peripheral precues: Deconfounding the contributions of cue eccentricity, cue discrimination and spatial correspondence. Visual Cognition, 9, 303-336. 
Lambert, A., Naikar, N., McLachlan, K., \& Aitken, V. (1999). A new component of visual orienting: Implicit effects of peripheral information and subthreshold cues on covert attention. Journal of Experimental Psychology: Human Perception and Performance, $25,321-340$.

Lambert, A., Roser, M., Wells, I., \& Heffer, C. (2006). The spatial correspondence hypothesis and orienting in response to central and peripheral spatial cues. Visual Cognition, 13, 65-88.

Lau, H. C., \& Passingham, R. E. (2007). Unconscious Activation of the Cognitive Control System in the Human Prefrontal Cortex. The Journal of Neuroscience, 27(21), 58055811. doi:10.1523/JNEUROSCI.4335-06.2007

Lau, H., \& Rosenthal, D. (2011) Empirical support for higher-order theories of conscious awareness. Trends in Cognitive Science, 15, 365-373.

Lavie, N. (1995). Perceptual load as a necessary condition for selective attention. Journal of Experimental Psychology: Human Perception and Performance, 21, 451-468.

Lavie, N., \& Tsal, Y. (1994). Perceptual load as a major determinant of the locus of selection in visual attention. Attention, Perception, \& Psychophysics, 56, 183-197.

Leuthold, H., \& Kopp, B. (1998). Mechanisms of priming by masked stimuli: Inferences from event-related potentials. Psychological Science, 9, 263-269.

Lleras, A., \& Enns, J. T. (2004). Negative compatibility or object updating? a cautionary tale of mask-dependent priming. Journal of Experimental Psychology: General, 133 (4), 475-493. 
Loftus, G. R., \& Masson, M. E. J. (1994). Using confidence intervals in within-subject designs. Psychonomic Bulletin \& Review, 1(4), 476-490. doi:10.3758/BF03210951

Logan, G. D., \& Bundesen, C. (2003). Clever homunculus: Is there an endogenous act of control in the explicit task-cuing procedure? Journal of Experimental Psychology: Human Perception and Performance, 29(3), 575-599. doi:10.1037/00961523.29.3.575

Luck, S. J. (1995). Multiple mechanisms of visual-spatial attention: recent evidence from human electrophysiology. Behavioural Brain Research, 71(1-2), 113-123. doi:10.1016/0166-4328(95)00041-0

Luck, S. J. (1998). Sources of Dual-Task Interference: Evidence From Human Electrophysiology. Psychological Science, 9(3), 223-227. doi:10.1111/14679280.00043

Luck, S. J., Heinze, H. J., Mangun, G. R., \& Hillyard, S. A. (1990). Visual event-related potentials index focused attention within bilateral stimulus arrays. ii. functional dissociation of p1 and $\mathrm{n} 1$ components. Electroencephalography and clinical Neurophysiology, 75, 528-542.

Luck, S. J., \& Hillyard, S. A. (1995). The role of attention in feature detection and conjunction discrimination: an electrophysiological analysis. The International journal of neuroscience, 80(1-4), 281-297. Retrieved from http://informahealthcare.com/loi/nes

Luck, S. J., \& Hillyard, S. A. (2000). The operation of selective attention at multiple stages of processing: Evidence from human and monkey electrophysiology. In M. S. Gazzaniga (Ed.), The new cognitive neurosciences (pp. 687-700). Cambridge MA: MIT Press 
Luck, S. J., Hillyard, S. A., Mouloua, M., \& Hawkins, H. L. (1996). Mechanisms of visualspatial attention: Resource allocation or uncertainty reduction? Journal of Experimental Psychology: Human Perception and Performance, 22(3), 725-737. doi:10.1037/0096-1523.22.3.725

Luck, S. J., Hillyard, S. A., Mouloua, M., Woldorff, M. G., Clark, V. P., \& Hawkins, H. L. (1994). Effects of spatial cuing on luminance detectability: Psychophysical and electrophysiological evidence for early selection. Journal of Experimental Psychology: Human Perception and Performance, 20(4), 887-904. doi:10.1037/00961523.20.4.887

Luck, S. J., Woodman, G. F., \& Vogel, E. K. (2000). Event-related potential studies of attention. Trends in Cognitive Sciences, 4(11), 432-440. doi:10.1016/S13646613(00)01545-X

Macmillan, N. A., \& Creelman, C. D. (1991). Signal detection theory: a user's guide. Cambridge: Cambridge University Press.

Mangun, G. R., \& Hillyard, S. A. (1991). Modulations of sensory-evoked brain potentials indicate changes in perceptual processing during visual-spatial priming. Journal of Experimental Psychology: Human Perception and Performance, 17(4), 1057-1074. doi:10.1037/0096-1523.17.4.1057

Marzouki, Y., Grainger, J., \& Theeuwes, J. (2007). Exogenous spatial cueing modulates subliminal masked priming. Acta Psychologica, 126(1), 34-45. doi:10.1016/j.actpsy.2006.11.002

Mattler, U. (2003a). Combined perceptual or motor-related expectancies modulated by type of cue. Perception \& Psychophysics, 65, 649-666. 
Mattler, U. (2003b). Priming of mental operations by masked stimuli. Perception \& Psychophysics, 65(2), 167-187. doi:10.3758/BF03194793

Mattler, U. (2004). Combined expectancy effects are modulated by the relation between expectancy cues. Quarterly Journal of Experimental Psychology, 57A, 193-221.

Mattler, U. (2005). Inhibition and decay of motor and nonmotor priming. Perception \& Psychophysics, 67(2), 285-300. doi:10.3758/BF03206492

Mattler, U. (2006). On the locus of priming and inverse priming effects. Perception \& Psychophysics, 68(6), 975-991.

Mattler, U. (2007). Inverse target- and cue-priming effects of masked stimuli. Journal of Experimental Psychology: Human Perception and Performance, 33(1), 83-102. doi:10.1037/0096-1523.33.1.83

Mattler, U., \& Fendrich, R. (2007). Priming by motion too rapid to be consciously seen. Attention, Perception, \& Psychophysics, 69(8), 1389-1398. doi:10.3758/BF03192954

Mattler, U., \& Palmer, S. (2012). Time course of free choice priming effects explained by a simple accumulator model. Cognition, 123, 347-360.

McCormick, P. A. (1997). Orienting attention without awareness. Journal of Experimental Psychology: Human Perception and Performance, 23, 168-180.

McDonald, J. J., Teder-Sälejärvi, W. A., \& Hillyard, S. A. (2000). Involuntary orienting to sound improves visual perception. Nature, 407(6806), 906-908. doi: $10.1038 / 35038085$ 
Mulckhuyse, M., Talsma, D., \& Theeuwes, J. (2007). Grabbing attention without knowing: Automatic capture of attention by subliminal spatial cues. Visual Cognition, 15, 779788.

Mulckhuyse, M., \& Theeuwes, J. (2010). Unconscious attentional orienting to exogenous cues: A review of the literature. Acta Psychologica, 134, 299-309.

Müller, H. J., \& Humphreys, G. W. (1991). Luminance-increment detection: Capacity-limited or not? Journal of Experimental Psychology: Human Perception and Performance, $17(1), 107-124$.

Müller, H. J., \& Rabbitt, P. M. A. (1989). Reflexive and voluntary orienting of visual attention: Time course of activation and resistance to interruption. Journal of Experimental Psychology: Human Perception and Performance, 15, 315-330.

Naccache, L., Blandin, E., \& Dehaene, S. (2002). Unconscious Masked Priming Depends on Temporal Attention. Psychological Science, 13(5), 416-424. doi:10.1111/14679280.00474

Neumann, O. (1990). Direct parameter specification and the concept of perception. Psychological Research, 52, 207-215.

Neumann, O., \& Klotz, W. (1994). Motor responses to nonreportable, masked stimuli: Where is the limit of direct parameter specification? In Umiltā, C. (1994). Attention and Performance XV: Conscious and Nonconscious Information Processing. (pp. 123150). Cambridge, MA, US: The MIT Press.

Nicholson, R., Karayanidis, F., Bumak, E., Poboka, D., \& Michie, P. T. (2006). ERPs dissociate the effects of switching task sets and task cues. Brain Research, 1095(1), 107-123. doi:10.1016/j.brainres.2006.04.016 
Palmer, S., \& Mattler, U. (2013a). Masked stimuli modulate endogenous shifts of spatial attention. Consciousness and Cognition, 22, 486-503.

Palmer, S., \& Mattler, U. (2013b). On the source and scope of priming effects of masked stimuli on endogenous shifts of spatial attention. Consciousness and Cognition, 22, $528-544$.

Peelen, M. V., Heslenfeld, D. J., \& Theeuwes, J. (2004). Endogenous and exogenous attention shifts are mediated by the same large-scale neural network. NeuroImage, 22(2), 822830. doi:10.1016/j.neuroimage.2004.01.044

Periáñez, J. A., \& Barceló, F. (2009). Updating sensory versus task representations during task-switching: Insights from cognitive brain potentials in humans. Neuropsychologia, 47(4), 1160-1172. doi:10.1016/j.neuropsychologia.2009.01.014

Posner, M. I. (1980). Orienting of attention. Quarterly Journal of Experimental Psychology, $32,3-25$.

Posner, M. I. (1994). Attention: The Mechanisms of Consciousness. Proceedings of the National Academy of Sciences, 91(16), 7398-7403. doi:10.1073/pnas.91.16.7398

Posner, M. I., \& Cohen, Y. (1984). Components of visual orienting. In D. Bouma \& D. Bonwhuis (Eds.), Attention and Performance X (pp. 531-556). Hillsdale, NJ: Erlbaum.

Posner, M. I., Cohen, Y., \& Rafal, R. D. (1982). Neural systems control of spatial orienting. Philosophical Transactions of the Royal Sociaty of London B, 298, 187-198.

Posner, M. I., Walker, J. A., Friedrich, F. J., \& Rafal, R. D. (1984). Effects of parietal injury on covert orienting of attention. The Journal of Neuroscience, 4(7), 1863-1874.

Pratkanis, A. R. (1992). The cargo-cult sciece of subliminal persuation. Skeptical Inquirer, $16,260-272$. 
Prinzmetal, W., McCool, C., \& Park, S. (2005). Attention: Reaction Time and Accuracy Reveal Different Mechanisms. Journal of Experimental Psychology: General, 134(1), 73-92. doi:10.1037/0096-3445.134.1.73

Rahnev, D., Maniscalco, B., Graves, T., Huang, E., de Lange, F., \& Lau, H. (2011). Attention induces conservative subjective biases in visual perception. Nature Neuroscience, 14 , 1513-1515.

Reuss, H., Kiesel, A., Kunde, W., \& Hommel, B. (2011). Unconscious activation of task sets. Consciousness \& Cognition, 20, 556-567.

Reuss, H., Pohl, C., Kiesel, A., \& Kunde, W. (2011). Follow the sign! Top-down contingent attentional capture of masked arrow cues. Advances in Cognitive Psychology, 7, 8291.

Risko, E. F., \& Stolz, J. A. (2010). The proportion valid effect in covert orienting: strategic control or implicit learning? Consciousness and cognition, 19(1), 432-442. doi:10.1016/j.concog.2009.07.013

Ristic, J., \& Kingstone, A. (2006). Attention to arrows: Pointing to a new direction. The Quarterly Journal of Experimental Psychology, 59, 1921-1930.

Rizzolatti, G., Riggio, L., Dascola, I., \& Umilta, C. (1987). Reorienting attention across the horizontal and vertical meridians - evidence in favor of a premotor theory of attention. Neuropsychologia, 25, 31-40.

Sato, W., Okada, T., \& Toichi, M. (2007). Attentional shift by gaze is triggered without awareness. Experimental Brain Research, 183, 87-94.

Scharlau, I. \& Ansorge, U. (2003). Direct parameter specification of an attention shift: evidence from perceptual latency priming. Vision Research, 43, 1351-1363. 
Schlaghecken, F., \& Eimer, M. (2004). Masked prime stimuli can bias "free" choices between response alternatives. Psychonomic Bulletin \& Review, 11(3), 463-468. doi:10.3758/BF03196596

Schmidt, T. (2000). Visual perception without awareness: Priming responses by color. In T. Metzinger (Ed.), Neural Correlates of Consciousness: Empirical and Conceptual Questions (pp. 157-169). Cambridge, MA: MIT Press.

Schmidt, T. (2002). The finger in flight: Real-time motor control by visually masked color stimuli. Psychological Science, 13, 112-118.

Schmidt, T., \& Vorberg, D. (2006). Criteria for unconscious cognition: Three types of dissociation. Attention, Perception, \& Psychophysics, 68(3), 489-504. doi:10.3758/BF03193692

Schneider, D. W., \& Logan, G. D. (2006). Priming cue encoding by manipulating transition frequency in explicitly cued task switching. Psychonomic Bulletin \& Review, 13(1), 145-151. doi:10.3758/BF03193826

Shaw, M. L. (1984). Division of attention among spatial locations: A fundamental difference between detection of letters and detection of luminance increments. In H. Bouma \& D. G. Bonwhuis (Eds.), Attention and Performance X (pp. 109-121). Hillsdale, NJ: Erlbaum.

Shin, M.-J., Marrett, N., \& Lambert, A. J. (2011). Visual orienting in response to attentional cues: Spatial correspondence is critical, conscious awareness is not. Visual Cognition, 19(6), 730-761. doi:10.1080/13506285.2011.582053

Shiu, L., \& Pashler, H. (1994). Negligible effect of spatial precuing on identification of single digits. Journal of Experimental Psychology: Human Perception and Performance, 20, 1037-1054. 
Slagter, H. A., Kok, A., Mol, N., \& Kenemans, J. L. (2005). Spatiotemporal dynamics of topdown control: Directing attention to location and/or color as revealed by ERPs and source modeling. Cognitive Brain Research, 22, 333-348.

Störmer, V., McDonald, J. \& Hillyard, S. (2009). Crossmodal cueing of attention alters appearance and early cortical processing of visual stimuli. Proceedings of the National Academy of Sciences, 106, 22456-22461.

Swets, J. A. (1986). Form of empirical ROCs in discrimination and diagnostic tasks:

Implications for theory and measurement of performance. Psychological Bulletin, 99(2), 181-198. doi:10.1037/0033-2909.99.2.181

Tipples, J. (2002). Eye gaze is not unique: Automatic orienting in response to uninformative arrows. Psychonomic Bulletin \& Review, 9, 314-318.

Thoma, V., Davidoff, J.B., \& Hummel, J. (2007). Priming of plane-rotated objects depends on attention and view familiarity. Visual Cognition, 15, 179-210.

Tononi, G. (2004). An information integration theory of consciousness. BMC Neuroscience, $5,42$.

Treisman, A. (1998). Feature binding, attention and object perception. Philosophical Transactions of the Royal Society, London, B, 353, 1295-1306.

Treisman, A., \& Gelade, G. (1980) A feature integration theory of attention. Cognitive Psychology, 12, 97-136.

Tsuchiya, N., Block, N., \& Koch, C. (2012). Top-down attention and consciousness: comment on Cohen et al. Trends in Cognitive Sciences, 16(11), 527. doi:10.1016/j.tics.2012.09.004 
Van den Bussche, E., Hughes, G., Humbeeck, N. V., \& Reynvoet, B. (2010). The relation between consciousness and attention: An empirical study using the priming paradigm. Consciousness and Cognition, 19(1), 86-97. doi:10.1016/j.concog.2009.12.019

Van den Bussche, E., Van den Noortgate, W., \& Reynvoet, B. (2009). Mechanisms of masked priming: A meta-analysis. Psychological Bulletin, 135(3), 452-477. doi:10.1037/a0015329

Van Gaal, S., De Lange, F. P., \& Cohen, M. X. (2012). The role of consciousness in cognitive control and decision making. Frontiers in Human Neuroscience, 6. doi:10.3389/fnhum.2012.00121

Van Gaal, S., \& Lamme, V. A. F. (2012). Unconscious High-Level Information Processing Implication for Neurobiological Theories of Consciousness. The Neuroscientist, 18(3), 287-301. doi:10.1177/1073858411404079

van Gaal, S., Ridderinkhof, K. R., Fahrenfort, J. J., Scholte, H. S., \& Lamme, V. A. F. (2008). Frontal cortex mediates unconsciously triggered inhibitory control. Journal of Neuroscience, 28, 8053-8062.

van Gaal, S., Ridderinkhof, K. R., van den Wildenberg, W. P. M., \& Lamme, V. A. F. (2009). Dissociating consciousness from inhibitory control: Evidence for unconsciously triggered response inhibition in the stop-signal task. Journal of Experimental Psychology: Human Perception and Performance, 35, 1129-1139.

van Gaal, S., Scholte, H. S., Lamme, V. A. F., Fahrenfort, J. J., \& Ridderinkhof, K. R. (2011). Pre-SMA Gray-matter Density Predicts Individual Differences in Action Selection in the Face of Conscious and Unconscious Response Conflict. Journal of Cognitive Neuroscience, 23(2), 382-390. doi:10.1162/jocn.2010.21444 
Van Velzen, J., \& Eimer, M. (2003). Early posterior ERP components do not reflect the control of attentional shifts toward expected peripheral events. Psychophysiology, 40(5), 827-831. doi:10.1111/1469-8986.00083

Vogel, E. K., \& Luck, S. J. (2000). The visual N1 component as an index of a discrimination process. Psychophysiology, 37(02), 190-203. doi: 10.1111/1469-8986.3720190

Vogel, E. K., Luck, S. J., \& Shapiro, K. L. (1998). Electrophysiological evidence for a postperceptual locus of suppression during the attentional blink. Journal of Experimental Psychology: Human Perception and Performance, 24(6), 1656-1674. doi:10.1037/0096-1523.24.6.1656

Vorberg, D., Mattler, U., Heinecke, A., Schmidt, T., \& Schwarzbach, J. (2003). Different time courses for visual perception and action priming. Proceedings of the National Academy of Sciences, 100, 6275-6280.

Vorberg, D., Mattler, U., Heinecke, A., Schmidt, T., \& Schwarzbach, J. (2004). Invariant time course of priming with and without awareness. In C. Kaernbach, E. Schröger, \& H. Müller (Eds.), Psychophysics beyond sensation. Laws and invariants of human cognition (pp. 271-288). Mahwah, NJ: Lawrence Erlbaum Associates, Publishers.

Wolff, P. (1989, April). Einfluss des maskierten Testreizes auf die Wahlreaktion auf den Maskierreiz, bei Metakontrast [Effect of masked stimulus on choice response to thenmasking stimulus in metacontrast]. Paper presented at the 31st Tagung experimentell arbeitender Psychologen, Bamberg, Germany.

Yeshurun, Y., \& Carrasco, M. (1998). Attention improves or impairs visual performance by enhancing spatial resolution. Nature, 396, 72-75. 
Zhou, F. A., \& Davis, G. (2012). Unconscious priming of task sets: The role of spatial attention. Attention, Perception, \& Psychophysics, 74(1), 105-114. doi:10.3758/s13414-011-0221-8 


\section{List of Figures}

\section{Project 1 - Spatial cue-priming effects in letter discrimination tasks}

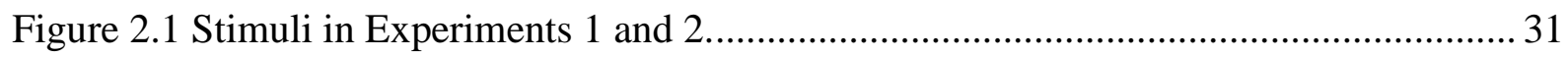

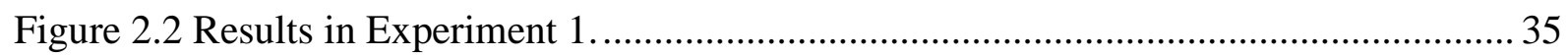

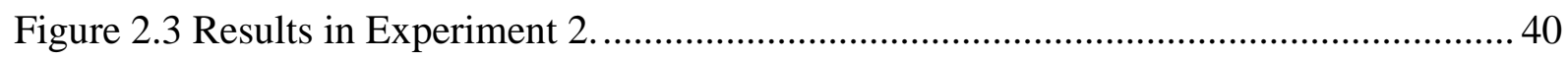

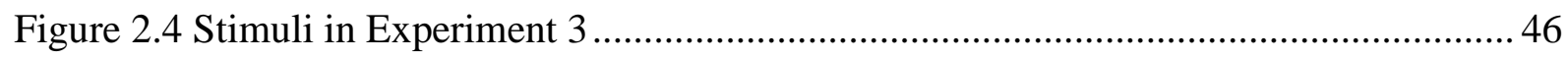

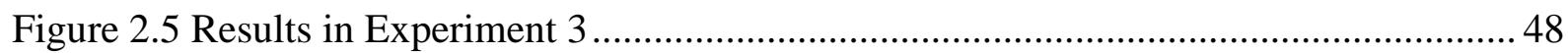

Figure 2.6 Prime recognition performance and priming effects on RT for two groups in

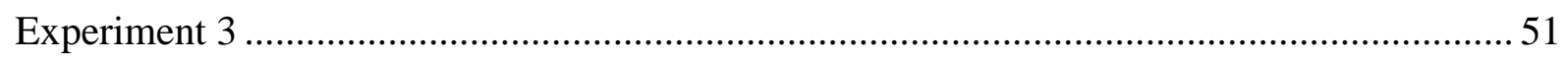

Project 2 - Spatial cue-priming effects on accuracy in signal detection tasks

Figure 3.1 Sequence of stimulus events in a trial of Experiment 1..................................... 79

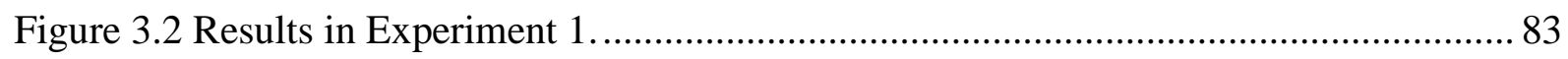

Figure 3.3 Scatterplots of priming effects an prime visibility in Experiment 1 ..................... 86

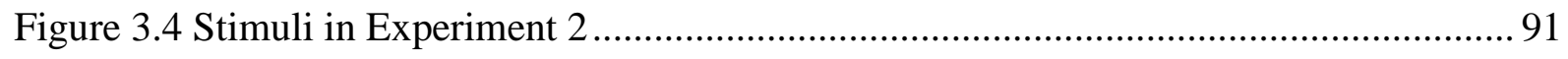

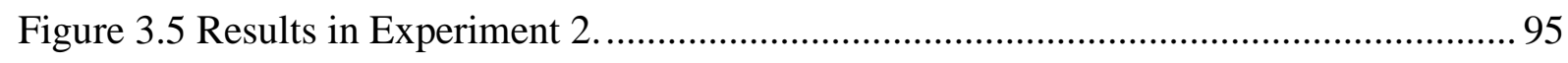

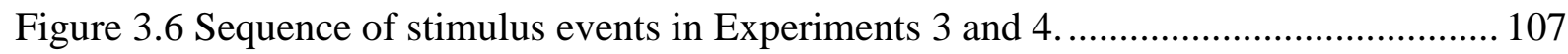

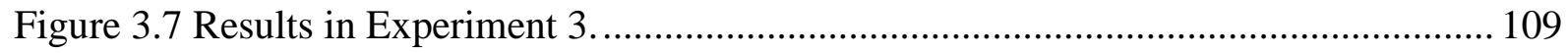

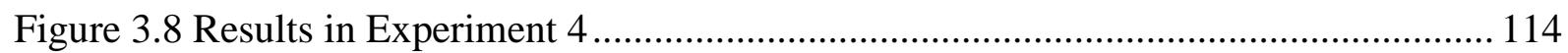

Project 3 - Spatial cue-priming effects on physiological measures of target processsing

Figure 4.1 Sequence of stimulus events in a trial of Experiment 1 ................................... 124

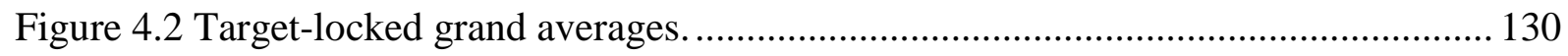

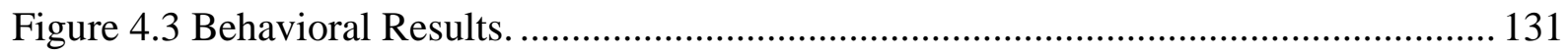

Figure 4.4 Mean amplitude of target-locked ERP components. ........................................ 133

Figure 4.5 Topographical distributions for priming effects ........................................... 134

Figure 4.6 The relation between prime visibility and priming effects ............................... 136

Figure 4.7 Sequence of stimulus events in a trial of Experiment 2 ................................. 145 


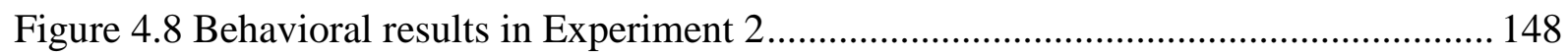

Figure 4.9 Probe locked potentials at PO7/PO8 .......................................................... 149

Figure 4.10 Probe - no probe difference potentials at PO7/PO8 …................................. 150

Figure 4.11 Voltage maps for the probe - no probe difference in Experiment 2.................. 151

Figure 4.12 Voltage maps for priming effects in target-locked ERPs in Experiment 2. ....... 152

Figure 4.13 Target-locked ERPs at PO7/PO8 for no probe trials. ..................................... 153

Figure 4.14 Target-locked potentials at FP1/FP2 for congruent and incongruent trials........ 155

Figure 4.15 Target-locked potentials at FC3/FC4 for no probe trials ................................. 156

Figure 4.16 Sequence of stimulus events in a trial of Experiment 3 ................................. 162

Figure 4.17 Behavioral results in Experiment 3 ........................................................ 165

Figure 4.18 Voltage maps for congruency effects in analyzed periods in Experiment 3. ..... 166

Figure 4.19 Target-locked ERPs at PO7/PO8 for trials with short SOA............................. 167

Figure 4.20 Target-locked ERPs at PO7/PO8 for trials with long SOA.............................. 169

Figure 4.21 Target-locked ERP differences between short SOA and long SOA at PO7/PO8.

\section{Project 4 - Free choice cue-priming}

Figure 5.1 Sequence of stimulus events in a trial of Experiment 1................................. 186

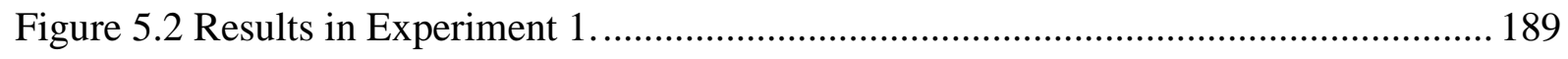

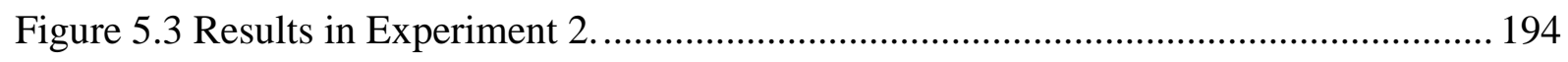

\section{Project 5 - The role of cue validity in spatial cue-priming}

Figure 6.1 Sequence of events in a trial of Experiment 1............................................ 200

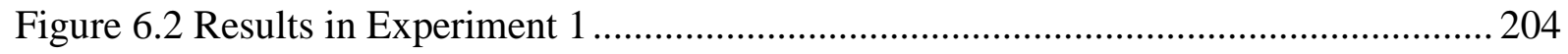

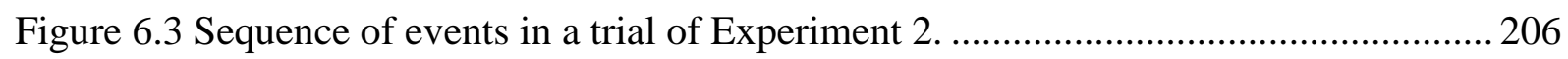

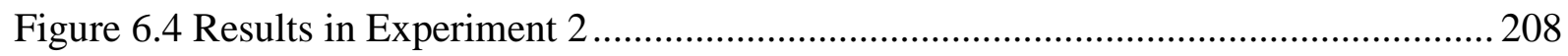




\section{List of Tables}

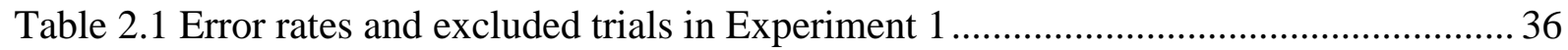

Table 2.2 Error rates and excluded trials in Experiment 2 ............................................... 41

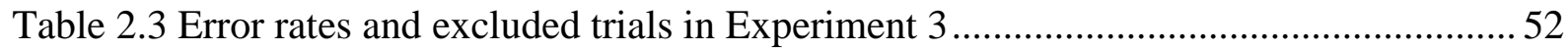

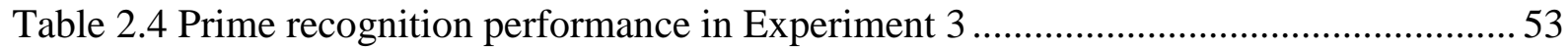

Table 3.1 Mean hit rates and false alarm rates and results of t-test in Experiment 1.............. 84

Table 3.2 Mean hit rates and false alarm rates and results of t-test in Experiment 2.............97 


\section{Curriculum Vitae}

\section{Persönliche Daten}

Name: $\quad$ Simon Palmer

Geburtsdatum: $\quad 21.03 .1985$

Geburtsort: $\quad$ Braunschweig

Anschrift: $\quad$ Maria-Montessori-Weg 9, 37073 Göttingen

\section{Ausbildung}

$05 / 2004$

Abitur, IGS Franzsches Feld, Braunschweig

10/2004 - 04/2009 Psychologiestudium an der Georg-August-Universität

\section{Göttingen}

$09 / 2006$

Vordiplom in Psychologie

$04 / 2009$

Diplom in Psychologie

05/2009 - 06/2012 wissenschaftlicher Mitarbeiter in der Abteilung für experimentelle Psychologie an der Georg-August-Universität

Göttingen (Promotion)

Göttingen, 07.02.2013 Simon Palmer 